\title{
WestVirginiaUniversity
}

THE RESEARCH REPOSITORY @ WVU

Graduate Theses, Dissertations, and Problem Reports

2010

\section{Mechanical degradation of thermal properties of flexible aerogel blankets}

Brice G. Gnahore

West Virginia University

Follow this and additional works at: https://researchrepository.wvu.edu/etd

\section{Recommended Citation}

Gnahore, Brice G., "Mechanical degradation of thermal properties of flexible aerogel blankets" (2010). Graduate Theses, Dissertations, and Problem Reports. 4596.

https://researchrepository.wvu.edu/etd/4596

This Dissertation is protected by copyright and/or related rights. It has been brought to you by the The Research Repository @ WVU with permission from the rights-holder(s). You are free to use this Dissertation in any way that is permitted by the copyright and related rights legislation that applies to your use. For other uses you must obtain permission from the rights-holder(s) directly, unless additional rights are indicated by a Creative Commons license in the record and/ or on the work itself. This Dissertation has been accepted for inclusion in WVU Graduate Theses, Dissertations, and Problem Reports collection by an authorized administrator of The Research Repository @ WVU.

For more information, please contact researchrepository@mail.wvu.edu. 


\title{
Mechanical Degradation of Thermal Properties of Flexible Aerogel Blankets
}

\author{
Brice G. Gnahore \\ Dissertation submitted to the \\ College of Engineering and Mineral Resources \\ at West Virginia University \\ in partial fulfillment of the requirements \\ for the degree of \\ Doctor of Philosophy
in
Mechanical Engineering \\ Committee members: \\ Darran Cairns, Ph.D., Chair \\ Ever Barbero, Ph.D. \\ Xingbo Liu, Ph.D. \\ Gergis William, Ph.D. \\ Konstantinos Sierros, Ph.D.
}

Department of Mechanical and Aerospace Engineering

\section{Morgantown, West Virginia \\ 2010}

Keywords: aerogel, insulation blanket, microstructure, weight loss , effective thermal conductivity 


\section{ABSTRACT \\ Mechanical Degradation of Thermal Properties of Flexible Aerogel Blankets Brice G. Gnahore}

Aerogel materials are of significant interest to numerous applications in many areas of engineering such as oil, gas, aerospace, military, construction, building and apparel because they offer insulating properties that outperform traditional materials by a factor ranging from 2 to 8 . Although an aerogel can be manufactured in a clamshell, sleeve or sheet shapes, the most versatile form is a blanket. Aerogel blankets are composites of silica aerogel particles dispersed in a reinforcing fiber matrix that turns the brittle aerogel into a durable and flexible insulating mat.

While aerogel blankets exhibit excellent thermal properties, they are still some concerns over dust mitigation when the blankets are flexed and deformed. The generation of dust is important because it can lead to a degradation of the insulating properties and is not ideal for the work environment during installation for some applications during subsequent uses. In this study, various experimental investigations such as thermal analysis, three-point bending tests and tensile tests have been performed to characterize the thermal insulation properties and degradation level of several commercially-available flexible aerogel blanket materials. The results are reported and discussed in this study. The microstructure of these aerogel blankets are also investigated for better understanding of the failure process. Knowledge of the thermal and mechanical properties are important for the optimization of the design for these heteregeneous materials.

Usage of insulating aerogel blankets in thermal protection systems and heat- 
sensitive environments, such as infrared suppression around engine and hot components applications, leads to accurate predictions of their thermal properties such as their effective thermal conductivity in such applications. As part of this study, simple theoretical and numerical models have been developed to predict the effective thermal conductivity of flexible aerogel blankets, which consist of fibers, aerogel particles and air-pockets. The theoretical models are thermal conductivity models as a function of aerogel, air-pocket and fiber volume fractions. In the numerical study, the effective thermal conductivity of the aerogel composites is computed with different aerogel particles to fiber volume ratios using the finite element method. The numerical analysis of thermal conductivity is conducted by generating 3D models of the microstructure of the aerogel blanket.

Interesting predictions concerning the effects of air-pocket and aerogel particles volume fractions are observed. For low aerogel particles content (less than 10\%), the results of the theoretical and the numerical models show good agreement but discrepancies are observed at high aerogel particles content. 


\section{Dedication}

This dissertation is dedicated to the loving memory of my father:

\section{GNAHORE SOHORE EDOUARD}

His life, work and thirst for knowledge continue to inspire me today. 


\section{ACKNOWLEDGMENTS}

My first, and most earnest, acknowledgment goes to my research advisor and committee chair, Dr. Darran Cairns, for his mentoring, guidance, friendship and continuous support throughout this research.

I would like to thank Dr. Barbero for his involvement and his interest to this research

project. Dr. Barbero took time from his busy schedule to provide me precious suggestions to this work.

I am extremely grateful for the contributions of Dr. Liu and Dr. William who brought their expertise of composite materials and finite element analysis respectively to this work.

I would like to express my appreciation to Dr. Konstatinos Sierros for being always available for discussions and suggestions.

Thank you to all the committee members. Special thanks to my colleagues of the research group, who provided valuable comments about this work.

I also gratefully acknowledge the financial support of the Ivory Coast Ministry of Higher Education and Research through a scholarship.

My final, and most heartfelt, acknowledgment goes to my mother, my beloved wife Julie and my two children Viera Jorelle and Elysee-Frejus for their support, love, patience and their encouragement during my long journey for the $\mathrm{PhD}$ degree. 


\section{TABLE OF CONTENTS}

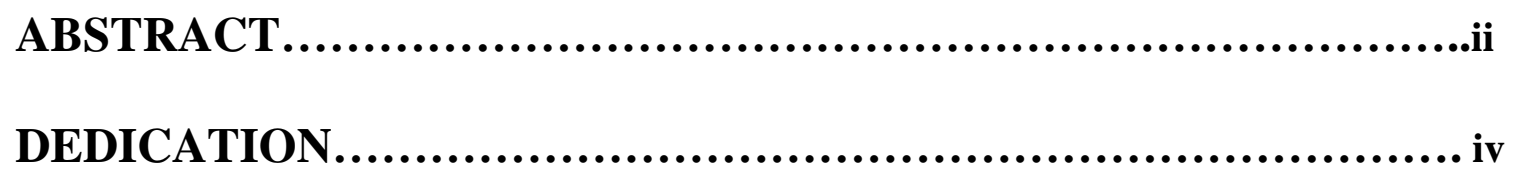

ACKNOWLEDGMENTS ...............................................

TABLE OF CONTENTS.................................................vi

LIST OF TABLES.................................................. ix

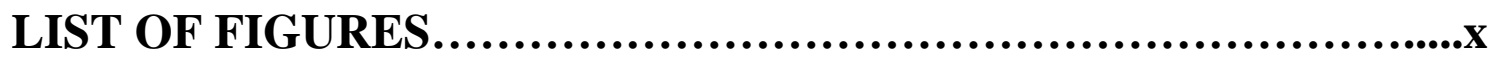

ABBREVIATIONS........................................................

NOMENCLATURES.................................................. xvii

\section{INTRODUCTION....................................................... 1}

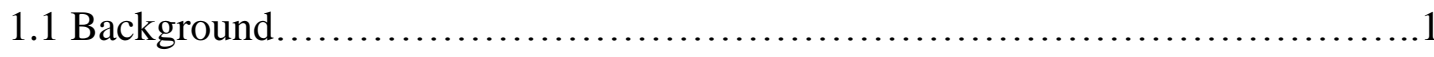

1.2 Problem Statement.............................................................. 5

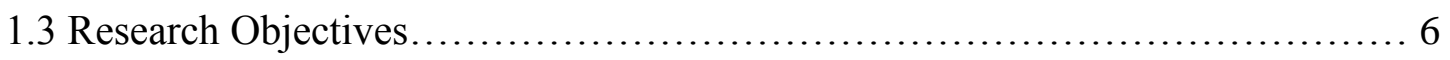

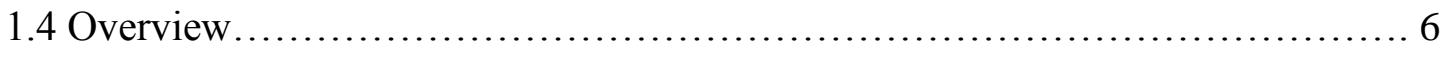

2. LITTERATURE REVIEW .......................................... 8

2.1 Introduction to Sol-Gel Science.................................................. 8

2.1.1 Hydrolysis and Condensation Reactions................................ 9

2.1.2 Gelation......................................................... 10

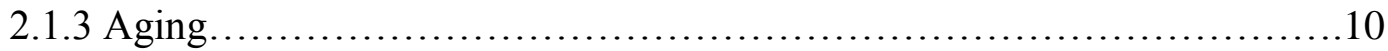

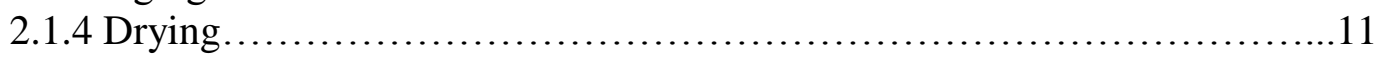

2.1.5 Supercritical drying .................................................... 12

2.2 Flexible aerogel blanket fabrication..........................................

2.3 Mechanical properties of a flexible aerogel blanket...........................17

2.3.1 Mechanical properties of aerogel.................................... 17

2.3.2 Mechanical degradation of aerogel ................................. 18

2.3.3 Mechanical behavior of an aerogel blanket...........................21 
2.4 Thermal properties of flexible aerogel blanket.............................22

2.4.1 Thermal conductivity of aerogel......................................22

2.4.2 Thermal conductivity of aerogel blanket.............................25

2.5 Computer simulation of aerogel............................................27

2.5.1 Computer modeling of mechanical structure property relationship

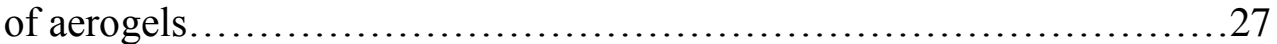

2.5.2 Finite element analysis of heat transfer in aerogel...................... 30

\section{EXPERIMENTAL PROCEDURES......................................................34}

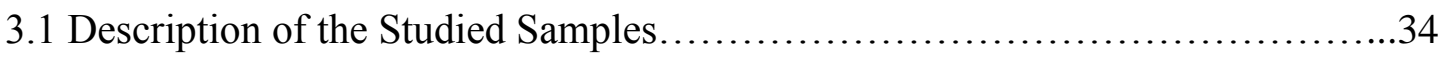

3.1.1 Spaceloft...................................................... 35

3.1.2 Pyrogel.......................................................... 37

3.2 Scanning Electronic Microscopy .......................................... 38

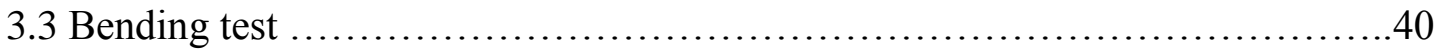

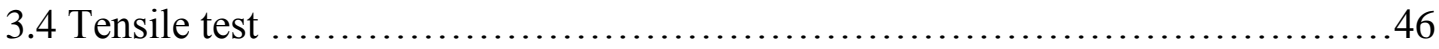

3.5 Measurement of thermal resistance ........................................47

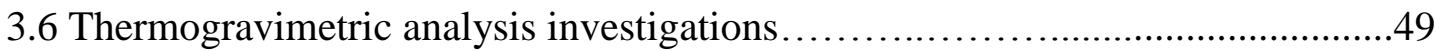

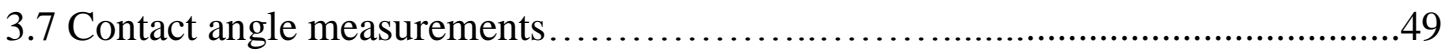

3.8 Numerical modeling using input from experimental observations....................50

\section{EXPERIMENTAL RESULTS ...................................52}

4.1 SEM Results ..............................................................

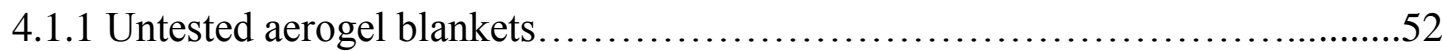

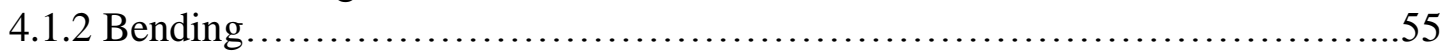

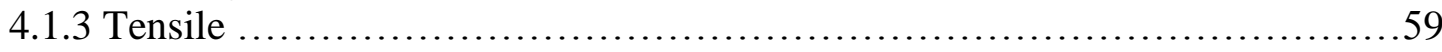

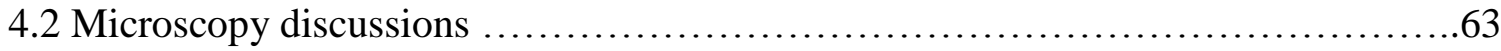

4.3 Flexural test results and discussions................................................66

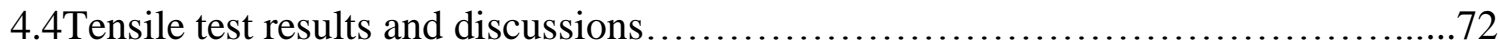

4.5 Results and discussions of thermal resistance measurements ..................77

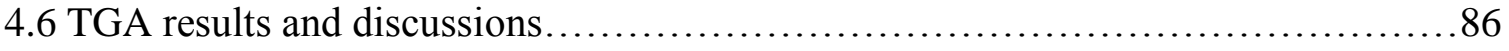

4.6.1 Volume percent of fiber and aerogel determination by TGA................94 


\section{THEORETICAL MODELS OF EFFECTIVE THERMAL} CONDUCTIVITY OF FLEXIBLE AEROGEL BLANKET .

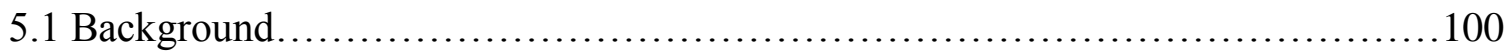

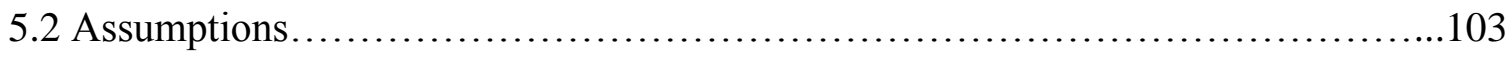

5.3Thermal Conductivity of the Constituents of the Aerogel Composites................104

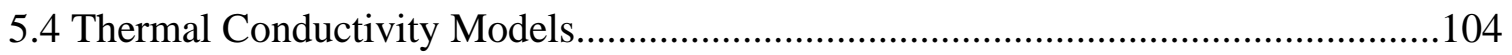

5.5 Results and Discussions.................................................... 111

6. FINITE ELEMENT ANALYSIS.................................116

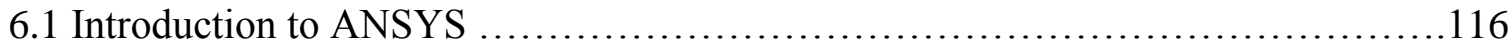

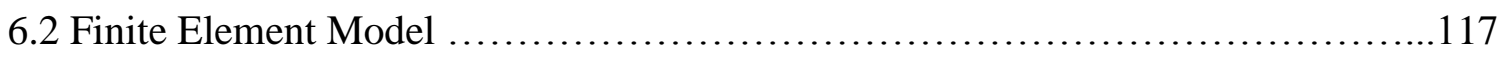

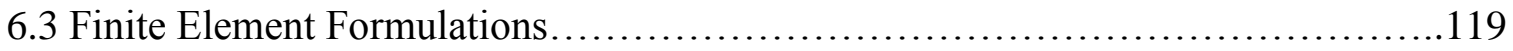

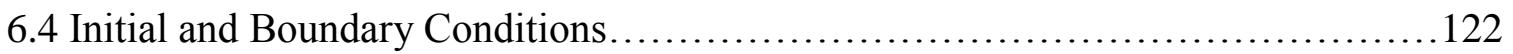

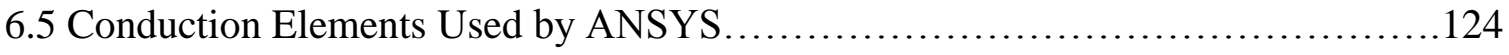

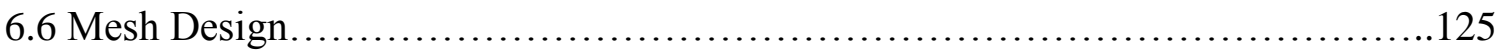

6.7 Modeling the Heat Transfer of flexible aerogel blankets ........................126

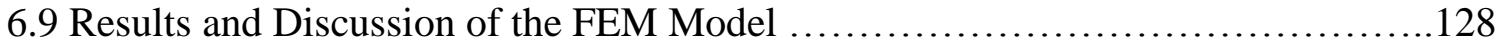

6.10 Comparison of the Numerical and Theoretical Models...........................141

7. CONCLUSION.....................................................143

8. RECOMMENDATIONS FOR FUTURE WORK..................148

REFERENCES.......................................................150 
APPENDIX: ANSYS Program Code for Thermal Simulation..........160 


\section{LIST OF TABLES}

Table 1.1 Evolution of aerogel production Speed $\ldots \ldots \ldots \ldots \ldots \ldots \ldots \ldots \ldots \ldots \ldots \ldots . \ldots \ldots$

Table 2.1 Characteristics of aerogel samples measured .............................24

Table 2.2 Chemical composition of some aerogel blanket samples.................26

Table 3.1 Properties of flexible aerogel blanket................................. 34

Table 4.1 Three point bending data of testing flexible aerogel blankets........................................................

Table 4.2 Tensile data of testing flexible aerogel blanket........................... 74

Table 4.3 Pyrogel blanket components volume fraction determination................96

Table 4.4 Spaceloft blanket components volume fraction determination...............96

Table 4.5 Contact angle between water drop and aerogel blanket surfaces..............99

Table 5.1 Data windows of SEM image...................................102-103

Table 5.2 Values of properties of the constituent materials.........................106 


\section{LIST OF FIGURES}

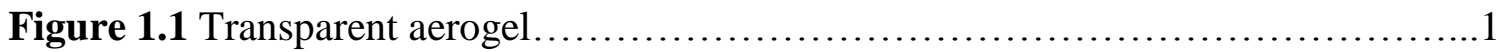

Figure 1.2 Historical development of aerogel......................................

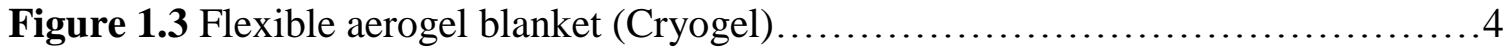

Figure 2.1 Fabrication mechanisms of aerogel........................................ 8

Figure 2.2 Schematic of the mold and press configuration for aerogel processing ................................................

Figure 2.3 General fabrication process of a flexible aerogel blanket...................15

Figure 2.4 Flowchart of the preparation of flexible aerogel blanket...................16

Figure 2.5 Representative stress-strain curves for aerogels under

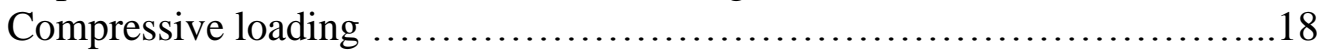

Figure 2.6 Logarithmic plot of stress versus the stress rate.........................20

Figure 2.7 Schematic of experimental set up...................................21

Figure 2.8 Variation of the compressive strain of aerogel blanket samples

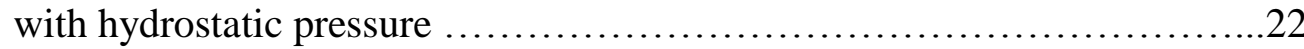

Figure 2.9 Variation of thermal conductivity with density at different temperatures.................................................

Figure 2.10 Total Thermal Conductivity of Aerogel Powder Samples versus Temperature for an External Load of $1.5 \times 10^{4} \mathrm{~Pa}$

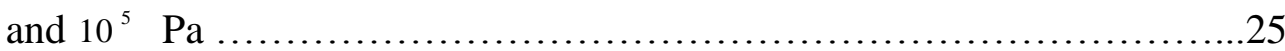

Figure 2.11 Thermal performance of some aerogel blankets samples................27

Figure 2.12 Cumulative distribution of strain energy in a bond......................29

Figure 2.13 Model cross-sectional dimensions of the solid, hollow and 4DG fibers..........................................................

Figure 2.14 Effective thermal conductivity of insulation materials...................32

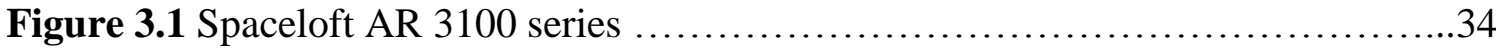




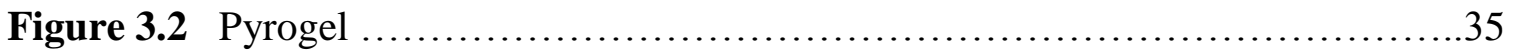

Figure 3.3 Spaceloft material insulation for hot water pipes........................36

Figure 3.4 Thermal images of a helicopter without

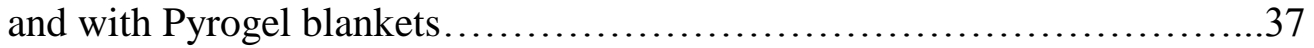

Figure 3.5 Large insulated vessel with Pyrogel thermal insulation .....................38

Figure 3.6 Photograph of scanning electron microscope Hitachi S-4000 ................39

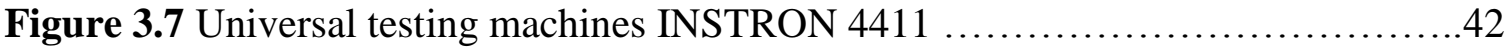

Figure 3.8 Behavior of elastic beam in bending ...................................42

Figure 3.9 Fisher Scientific Isotemp Digital hotplate ............................47

Figure 4.1 SEM images of untested Pyrogel blanket materials.......................52-54

Figure 4.2 SEM images of untested Spaceloft blanket materials....................54-55

Figure 4.3 SEM images of three points bending Pyrogel material surfaces..............56-58

Figure 4.4 SEM images of three points bending Pyrogel material surfaces ..........58-59

Figure 4.5 SEM images of tensile Pyrogel material surfaces.......................59-61

Figure 4.6 SEM images of tensile Spaceloft material surfaces.........................62

Figure 4.7 Engineering stress-strain curves of Pyrogel specimens..................66-67

Figure 4.8 Engineering stress-strain curves of Spaceloft specimens ................67

Figure 4.9 Graph of weight loss versus cycles for Pyrogel specimens ................70

Figure 4.10 Graph of weight loss versus cycles for Spaceloft specimens ..............71

Figure 4.11 Engineering stress-strain curves of Pyrogel specimens...................72

Figure 4.12 Engineering stress-strains curves of Spaceloft specimens................72

Figure 4.13 Graph of weight loss versus displacement of Pyrogel specimens.................76

Figure 4.14: Graph of weight loss versus displacement of Spaceloft specimens.........77

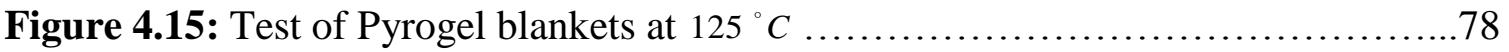




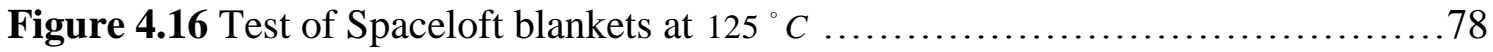

Figure 4.17 Thermal test at steady temperatures of Pyrogel blankets..................79

Figure 4.18 Thermal test at steady temperatures of Spaceloft blankets.................80

Figure 4.19 Normalized relative temperature drop of Pyrogel blankets.................81

Figure 4.20 Normalized relative temperature drop of Spaceloft blankets..............81

Figure 4.21 Temperature difference across Pyrogel blankets at $T_{\text {hopplate }}=150{ }^{\circ} \mathrm{C}$ under the flexural test.......................................................................... $82-83$

Figure 4.22 Temperature difference across Spaceloft blankets at $T_{\text {hoplate }}=150{ }^{\circ} \mathrm{C}$

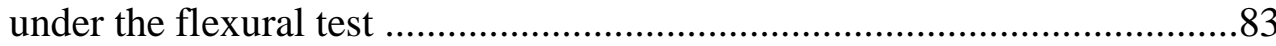

Figure 4.23 Temperature difference across Pyrogel blankets at $T_{\text {hopplate }}=75^{\circ} \mathrm{C}$ under

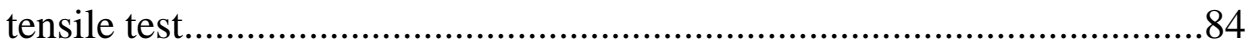

Figure 4.24 Temperature difference across Spaceloft blankets at $T_{\text {hopplate }}=75^{\circ} \mathrm{C}$

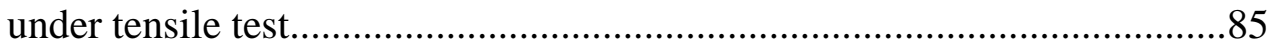

Figure 4.25 TGA thermograms of Pyrogel blanket materials in air....................87

Figure 4.26 TGA thermograms of Spaceloft blanket materials in air..................87

Figure 4.27 TGA thermograms of Pyrogel blanket materials in nitrogen...............88

Figure 4.28 TGA thermograms of Spaceloft blanket materials in nitrogen.............88

Figure 4.29 Isothermal TGA thermograms of Pyrogel blanket materials

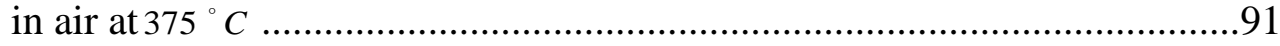

Figure 4.30 Isothermal TGA thermograms of Spaceloft blanket materials

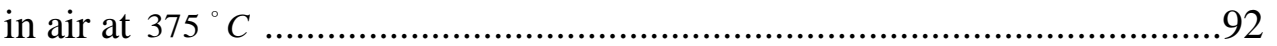

Figure 4.31 Isothermal TGA thermograms of Pyrogel blanket materials in nitrogen at $375^{\circ} \mathrm{C}$ 93

Figure 4.32 Isothermal TGA thermograms of Spaceloft blanket materials in nitrogen at $375^{\circ} \mathrm{C}$

Figure 4.33 Water drop on Pyrogel blanket surfaces. $. .97-98$ 
Figure 4.34: Water drop on Spaceloft blanket surfaces........................98

Figure 5.1: SEM image of a flexible aerogel blanket AR 5400...................101

Figure 5.2: Quantitative analysis of an SEM image of a flexible aerogel blanket AR 5400 102

Figure 5.3 Flexible aerogel blanket: parallel and series structures $106-107$

Figure 5.4 Predicted thermal conductivity values for flexible aerogel blankets

Figure 5.5 Krischer model predicted thermal conductivity values for flexible aerogel blankets

Figure 5.6 EMT model predicted thermal conductivity values for flexible aerogel blankets

Figure 6.1 Schematic of the simplified flexible aerogel blanket structure

Figure 6.2 Solid 70, Solid 90. 124

Figure 6.3 FEM mesh of an aerogel blanket. .126

Figure 6.4 Thermal loads of an aerogel blanket $127-128$

Figure 6.5 FEM mesh of fiber with no aerogel 129

Figure 6.6 Distribution of temperature over fiber with no aerogel..................130

Figure 6.7 Distribution of heat flux over fiber with no aerogel....................130

Figure 6.8 FEM mesh of fiber with $0.05 \mathrm{VF}$ aerogel

Figure 6.9 Distribution of temperature over fiber with $0.05 \mathrm{VF}$ aerogel

Figure 6.10 Distribution of heat flux over fiber with $0.05 \mathrm{VF}$ aerogel.

Figure 6.11 FEM mesh of fiber with $0.15 \mathrm{VF}$ aerogel 133

Figure 6.12 Distribution of temperature over fiber with $0.15 \mathrm{VF}$ aerogel 
Figure 6.13 Distribution of heat flux over fiber with $0.15 \mathrm{VF}$ aerogel................................................. 134

Figure 6.14 FEM mesh of fiber with $0.25 \mathrm{VF}$ aerogel ..............................135

Figure 6.15 Distribution of temperature over fiber

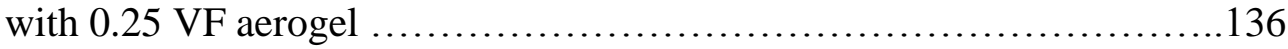

Figure 6.16 Distribution of heat flux over fiber with $0.25 \mathrm{VF}$ aerogel................................................ 136

Figure 6.17 FEM mesh of matrix with $0.4 \mathrm{VF}$ aerogel .............................137

Figure 6.18 Distribution of temperature over fiber

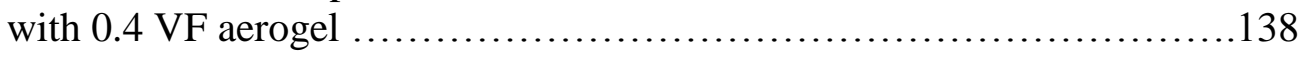

Figure 6.19 Distribution of heat flux over fiber with $0.4 \mathrm{VF}$ aerogel.................................................. 138

Figure 6.20 Variation of thermal conductivity with VF of aerogel...................139

Figure 6.21 Comparison of the FEM and theoretical results........................141 


\section{ABBREVIATIONS}

CCA - Cluster-Cluster Aggregation

$\mathrm{CO}_{2}$ - Carbon dioxide

DLA- Diffusion-Limited Aggregation

DLCA - Diffusion-Limited Cluster-cluster Aggregation

3-D - Three Dimensional

EMT- Effective Medium Theory

FE- Finite Element

FEM - Finite Element Modeling

HF- High Formamide

$\mathrm{H}_{2} \mathrm{O}$ - Water

IR- Infrared Radiation

LLNL - Lawrence Livermore National Laboratory

LNG- Liquefied Natural Gas

MEMS- Micro Electro Mechanical Systems

MMGBL - Microstructured Materials Group at Berkeley Laboratory

NASA - National Aeronautics and Space Administration

$\mathrm{Nu}-\mathrm{Nusselt}$ number

OH- Hydroxyl group

PET- Polyethylene

SEM - Scanning Electron Microscopy

TEOS- TetraEthylOrthoSilicate

TGA- ThermoGravimetric Analysis 
TMOS - TetraMethyOrthoSilicate

UTS- Ultimate Tensile Stress

VICTOR - Vacuum Insulation Conductivity Tester On Roller VF- Volume Fraction 


\section{NOMENCLATURE}

\section{Roman Symbols}

a- Slope of the initial straight line portion of the load deflection curves, N/mm

A- Cross sectional surface area, $m^{2}$

b- Width of the beam, mm

C - Heat capacity matrix

$C^{(e)}$ - Heat capacity matrix of external heat fluxes

$C_{p}$-Specific heat at constant pressure

D- Sample diameter, $\mathrm{mm}$

D- Maximum deflection of the center of the tested beam, $\mathrm{mm}$

d- Diameter of particles, $\mu_{m}$

$d_{0}$ - Base length of the droplet, $\mathrm{mm}$

${ }_{d} \theta$ - Infinitesimal angle of tested beam

E- Young's modulus, $\mathrm{MPa}$

$E_{B}-$ Modulus of elasticity of tested beam, $\mathrm{Pa}$

F- Nodal vector matrix

$F^{*}$ - Maximum Load inducing the fracture, $\mathrm{N}$

$F_{i}-$ Load at a selected point, $\mathrm{N}$

$F^{(e)}$ - Nodal vector of external heat fluxes matrix

h- Depth of tested beam, mm

$h_{0}$ - Height of the droplet, $\mathrm{mm}$

$h_{a}$ - Convective heat transfer coefficient of air, $\mathrm{W} / \mathrm{m}^{2}{ }^{\circ} \mathrm{C}$ 
I- Moment of inertia of the tested beam, $\mathrm{kg} \mathrm{m}^{2}$

$I_{y}-$ Moment of inertia of the tested beam in y direction, $\mathrm{kg} \mathrm{m} \mathrm{m}^{2}$

$I_{z}$ - Moment of inertia of the tested beam in $\mathrm{z}$ direction, $\mathrm{kg} \mathrm{m} \mathrm{m}^{2}$

K- Thermal conductivity matrix

$k_{\text {aerogel }}-$ Thermal conductivity of aerogel, W/m-K

$k_{\text {air }}$ - Thermal conductivity of air, W/m-K

$k_{\text {airconduct ion }}$ - Conductive thermal conductivity of air, W/m-K

$k_{\text {cond }}$ - The average value of the conductive thermal conductivity, W/m-K

$K^{(e)}$ - Thermal conductivity matrix of external heat fluxes

$k_{e 1}$ - Effective thermal conductivity of aerogel and fiber, W/m-K

$k_{f a b}$ - Thermal conductivity of flexible aerogel blanket, W/m-K

$k_{\text {fiber }}-$ Thermal conductivity of fiber, W/m-K

$k_{g}$ - Gas thermal conductivity, W/m-K

$k_{i j}$ - Tensor of thermal conductivity, W/m-K

$k_{\text {matrix }}$ - Thermal conductivity of matrix, W/m-K

$k_{r}$ - Radiative thermal conductivity, W/m-K

$k_{s}$ - Solid thermal conductivity, W/m-K

$k_{\text {total }}$ - Total effective thermal conductivity, W/m-K

$k_{x}$ - Thermal conductivity in $\mathrm{x}$ direction, $\mathrm{W} / \mathrm{m}-\mathrm{K}$

$k_{y}$ - Thermal conductivity in $\mathrm{x}$ direction, $\mathrm{W} / \mathrm{m}-\mathrm{K}$ 
$k_{z}$ - Thermal conductivity in $\mathrm{x}$ direction, $\mathrm{W} / \mathrm{m}-\mathrm{K}$

L- Span, mm

$l_{i}$ - Length at a selected point, $\mathrm{mm}$

$l_{0}$ - Initial length, $\mathrm{mm}$

$m_{i}$-mass of the specimen at a selected point, $\mathrm{mg}$

$m_{0}$ - Initial mass of the specimen, $\mathrm{mg}$

M- Maximum value of bending moment of the beam, N.mm

$M_{Z}$ - External moment of the beam, N.mm

n- Chemical susceptibility factor

$\mathrm{Nu}$ - Nusselt number

P-Pressure, $\mathrm{Pa}$

Pr- Prandtl number

Q- Heat, W

q- Heat flux into the fiber, $\mathrm{W} / \mathrm{m}^{2}$

$q_{i}$ - Heat flux at a selected point, $\mathrm{W} / \mathrm{m}^{2}$

$q_{x}$ - Heat flux in $\mathrm{x}$ direction, $\mathrm{W} / m^{2}$

$q_{y}$ - Heat flux in y direction, $\mathrm{W} / \mathrm{m}^{2}$

$q_{z}$ - Heat flux in z direction, $\mathrm{W} / \mathrm{m}^{2}$

$\mathrm{T}$ - Temperature, $\mathrm{K}$

17 - Temperature matrix

$T_{c . s .}$ - Temperature of the cold surface, ${ }^{0} \mathrm{C}$ 
$T_{h . s .}$ - Temperature of the hot surface, ${ }^{0} \mathrm{C}$

$\mathrm{u}$ - Velocity in $\mathrm{x}$ direction, $\mathrm{mm} / \mathrm{s}$

$v$-Velocity in y direction, $\mathrm{mm} / \mathrm{s}$

$V_{a i r}$ - Volume fraction of air

$V_{\text {aerogel }}-$ Volume fraction of aerogel

$V_{\text {fiber }}-$ Volume fraction of fiber

$V_{f a b}$ - Volume fraction of flexible aerogel blanket

w- Width of a sample, mm

$w$ - Velocity in $\mathrm{z}$ direction, $\mathrm{mm} / \mathrm{s}$

$\mathrm{x}, \mathrm{y}, \mathrm{z}-$ Spatial cartesian coordinates, $\mathrm{mm}$

$y$-Crosshead speed, $\mathrm{mm} / \mathrm{min}$

$y_{\max }$-Maximum deformation of beam axis, $\mathrm{mm}$

Z -Distribution factor

\section{Greek Symbols}

$\alpha$-Volumetric strain, $m^{3} / m^{3}$

$\delta$ - Thickness, mm

$\varepsilon$ - Engineering strain, $\mathrm{mm} / \mathrm{mm}$

$\varepsilon_{x}$ - Normal strain, $\mathrm{mm} / \mathrm{mm}$

$\varepsilon_{s}-$ Strain energy, J

$\Delta_{T}$ - Temperature difference across a sample, ${ }^{0} C$

$\Delta T_{\text {normalized }}$ - Normalized temperature drop, ${ }^{0} \mathrm{C}$ 
$\Delta T_{\max }$ - Maximum temperature difference across a sample, ${ }^{0} \mathrm{C}$

$\Delta y$ - Thickness of the blanket, $\mathrm{mm}$

$\phi$ - Flexible factor of EMT model

$\sigma-$ Engineering stress, $N / \mathrm{mm}^{2}$

$\dot{\sigma}$ - Stress rate, $\mathrm{Pa} / \mathrm{s}$

$\sigma_{c o}$-Maximum stress at the end of the depressurization process, Kpa

$\sigma_{R}$ - Modulus of rupture, Kpa

$\sigma_{x}$ - Normal longitudinal stress, $\mathrm{Pa}$

$\theta_{0}$ - Contact angle, degree Celsisus

$\rho$ - Radius of curvature, mm

$\rho^{m}$ - Density, $\mathrm{kg} / \mathrm{m}^{3}$

$\rho_{p}$ - Density of the Brownian particles, $\mathrm{kg} / \mathrm{m}^{3}$ 


\section{Chapter 1 Introduction}

\subsection{Background}

Recognized as the lightest and lowest density solid material $\left(0.003-0.35 \mathrm{~g} / \mathrm{cm}^{3}\right)$ by Guinness World Records [1-2], aerogels are unique materials with remarkable properties. Aerogels are less than 4 times as dense as dry air and less than 1,000 times as dense as glass and are highly porous with high surface areas $\left(500-1500 \mathrm{~m}^{2} / \mathrm{g}\right)$ [3]. These light and porous solid materials have gained much attention because of their insulating properties. Aerogels exhibit the lowest thermal conductivity $(0.011-0.013 \mathrm{~W} / \mathrm{m}-\mathrm{k})$, the lowest dielectric constant (1.1-2.2) and the lowest refractive index (1.025) values of any solid [4-6]. They have a semi-transparent nature and have been nicknamed "frozen smoke" or "solid smoke" due to their chemical composition of approximately $95 \%$ air and 5\% solid. Figure 1.1 shows a transparent aerogel material.

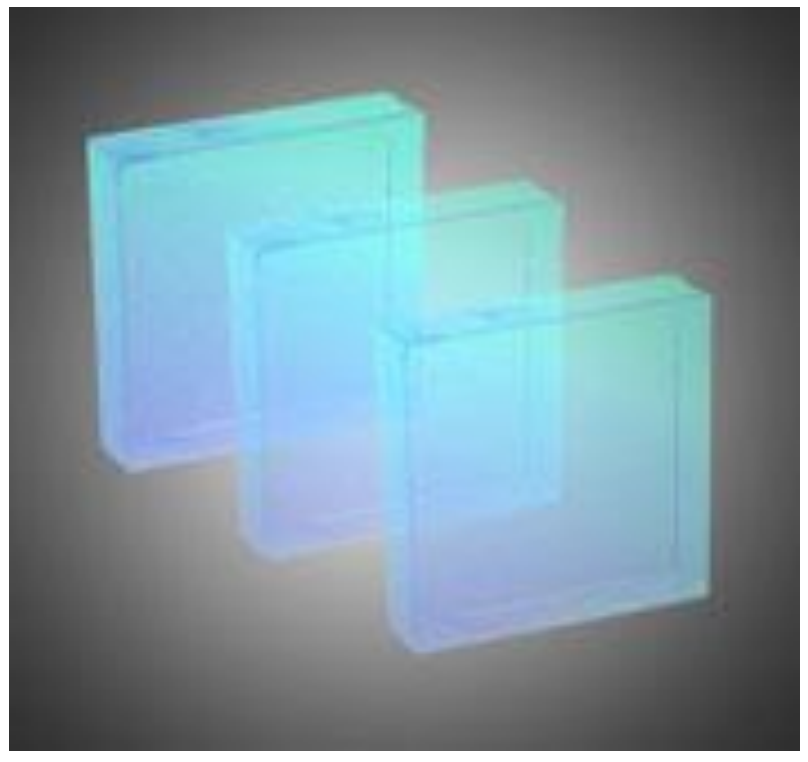

Figure 1.1: Transparent aerogel [7] 
The first aerogels were made in 1931 by Steven Kistler [8], a professor in chemical engineering at the College Pacific in California. Challenged by Charles Learned [9], Kistler replaced the liquid inside the wet gel with gas, without causing any shrinkage. This experiment led to the creation of the first aerogels made of silica. A few years later, Kistler made aerogels from alumina, chromia and tin [10]. In the early 1940s, Kistler was hired by Monsanto Corporation and helped them produce marketable granular silica aerogels known as "Santocel" [11].

In the late 1970s, Professor Stanislaus Teichner [12], of the University Claude Bernard of Lyon, was commissioned by French officials to investigate the use of aerogels for rocket fuel storage. Nicolaon [13], Teichner's student, prepared two aerogels in two weeks using Kistler's method of aerogel fabrication. Knowing that the project would require the preparation of a large number of aerogels in a short time, Teichner and Nicolaon used a new synthetic process to fabricate silica aerogels. An alkoxysilane TetraMethyOrthoSilicate (TMOS) was used instead of sodium silicate. Aerogels were made in one step by dissolving TMOS in a solution of methanol and hydrolyzing TMOS by water in the presence of either an acid or base catalyst. A few years later, a wide variety of metal oxide aerogels were prepared using this method. Indeed, the TMOS method was used to prepare detectors made of large tiles of silica aerogel. This leds to the use of aerogels as Cherenkov detectors in the early 1980s [14, 15]. Table 1.1 outlines time reduction in the preparation of aerogel. 
Table 1.1 Evolution of aerogel production speed [16]

\begin{tabular}{|l|l|}
\hline Year & $\begin{array}{l}\text { Production } \\
\text { time of } \\
\text { one batch }\end{array}$ \\
\hline 1930 's & 4 months \\
\hline 1970 's & 1 week \\
\hline 1990 's & 3 days \\
\hline 2002 & 4 hours \\
\hline 2004 & $\downarrow$ \\
\hline
\end{tabular}

The first international symposium on aerogels was organized in 1985 by Professor Jochen Fricke in Wurzburg, Germany [17]. The lowest density solid material, silica aerogel, was made in the Lawrence Livermore National Laboratory (LLNL) in the late 80 's. The density of this aerogel was measured as $0.003 \mathrm{~g} / \mathrm{cm}^{3}$. LLNL also made the first organic aerogels by using the same techniques as for inorganic aerogels [18]. In 1983, Microstructured Materials Group at Berkeley Laboratory (MMGBL) used Tetra Ethyl Ortho Silicate (TEOS) as a reagent instead of TMOS and carbon dioxide (liquid) instead of alcohol in the aerogels' preparation. They got a safer reaction and aerogels of a good quality [19]. In 1989, MMGBL discovered Thermaflux, which led to a preparation of a huge amount of aerogels [20]. Later, the MMGBL designed and built the Vacuum Insulation Conductivity Tester On Roller (VICTOR) to measure the thermal conductivity of large aerogel panels up to $26 \mathrm{~cm}$ on edge [21].

Furthermore, in February 1999, National Aeronautics and Space Administration (NASA) launched the Stardust spacecraft from Cape Canaveral, Florida. The main goal of the Stardust mission was to bring back to earth cometary samples and interstellar dust. To achieve this without damaging the comet particles, aerogels were used due to their porous structure to help dissipate the kinetic energy of the comet particles and their ability to catch the particles. The transparency (very high) and the color (smoky blue 
cast) of aerogels helped the scientists find the final position of the captured particles $[22,23]$.

In 2000, aerogel blankets were produced and commercialized by Aspen Aerogels, Inc .

Figure 1.2 summarizes the history and the evolution of aerogel.

Kistler prepared New synthetic process 1st international Thermalux was Stardust was launched the first aerogel of aerogel production Symposium on aerogel created by MMBL by NASA

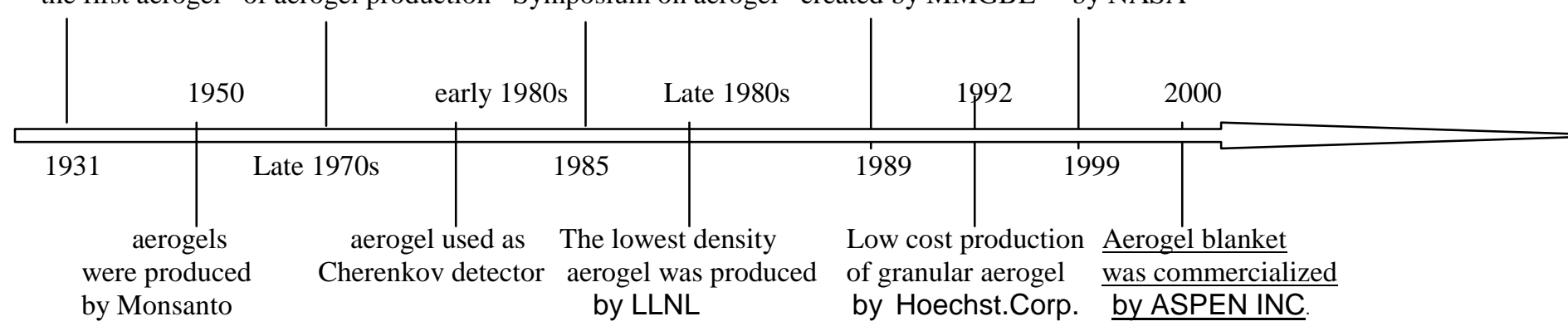

Figure 1.2: Historical development of aerogel

Aerogel blankets are engineered materials made from silica aerogel and reinforcing fibers. The brittle silica aerogel particles are incorporated into a polyester batting, creating a flexible and durable aerogel blanket. A flexible aerogel blanket is shown in figure 1.3 .

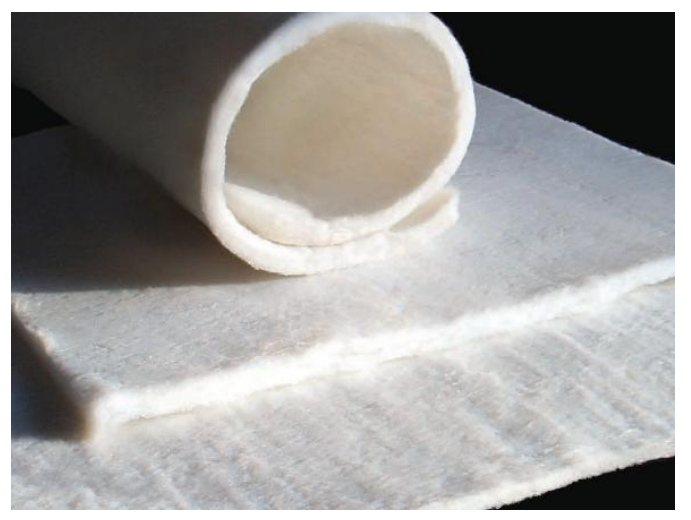

Figure 1.3: Flexible aerogel blanket (Cryogel) [24] 
Aerogel blankets are self-standing flexible materials. The flexibility of these blankets was achieved by putting a polymer batting firmly and deeply into the aerogel's molecular chain structure. A batting is a product obtained by the conversion of carding or garneting fiber into a soft web of fiber in sheet and non-sheet forms. It is a stuffing usually made of polyester, cotton and /or wool [25]. Aerogel blankets are lightweight, non-toxic and suitable for a broad range of applications in various areas such as oil, gas, aerospace, military, construction and building. They are used as insulators for low and high temperature application fields. Aerogel blankets are also used in applications that require flexibility, low density and ability to expand and to fill a void space [26].

\subsection{Problem Statement}

Aerogel blankets, although flexible, have little resistance to mechanical and thermal deformations, when they are subjected to various stress conditions. In fact, during the manufacturing and installation processes, the surface of aerogel blankets quickly release aerogel particles into the surrounding atmosphere upon application of a mechanical or thermal load. This event is called "dusting"[27]. Extensive dusting of fine aerogel particles leads to the collapse of the silica network of aerogel and thereby the degradation of the thermal properties of aerogel blankets. Aspen Aerogel Inc has developed flexible aerogel blankets with improved mechanical and thermal properties. In this study, various experimental investigations such as thermal analysis, three-point bending tests and tensile tests are performed to better understand the thermal insulation properties, degradation level and the correlations between the thermal and mechanical properties of these flexible aerogel blanket materials. The failure process of insulating blankets are analyzed by observing their microstructure. 
Flexible aerogel blankets exhibit good thermal properties because they can prevent heat from flowing through them by conduction or heat being transferred by convection. They have a useful applications in thermal protection systems and heat sensitive environments. For example in aerospace applications, flexible aerogel blankets provide thermal and fire protection for engine components [28]. This leads to an increased interest in accurately predicting their thermal properties such as their effective thermal conductivity in such applications. This study attempts to predict the effective thermal conductivity of flexible aerogel blankets.

\subsection{Research Objectives}

The objectives of this research are multifaceted. The first goal is to investigate the mechanical and thermal resistance of flexible aerogel blankets. The second is to study the degradation of aerogel blankets at the microstructure level. The third is to characterize the thermal insulation properties of aerogel blankets after mechanical degradation. The final objective is to develop theoretical and numerical models to predict the effective thermal conductivity of aerogel insulating blankets by evaluating the influence of the accumulated mechanical degradation on their thermal properties.

\subsection{Overview}

In chapter 2, previous works related to the fabrication and properties of aerogel blankets are discussed, in particular, thermal insulation of these blankets. Chapter 3 provides a description of aerogel blankets involved in this study and discusses the equipment and the procedures used to test the blankets. The results of the mechanical and thermal tests of the flexible aerogel blankets are analyzed in chapter 4. 
The theoretical and numerical micromechanical models of the aerogel blankets are presented in chapter 5 and 6 respectively. Chapter 7 provides a brief summary of the research and a general conclusion followed by some recommendations for future research in chapter 8 . 


\section{Chapter 2 Literature Review}

\subsection{Introduction to Sol-Gel}

Aerogel materials share a common preparation strategy with many composite materials. Indeed, aerogels are prepared via sol-gel processing involving the fabrication of colloidal suspensions also called sols which are converted to viscous gels and hence to solid materials [29]. The fabrication process is the result of different reaction mechanisms shown in figure 2.1 .

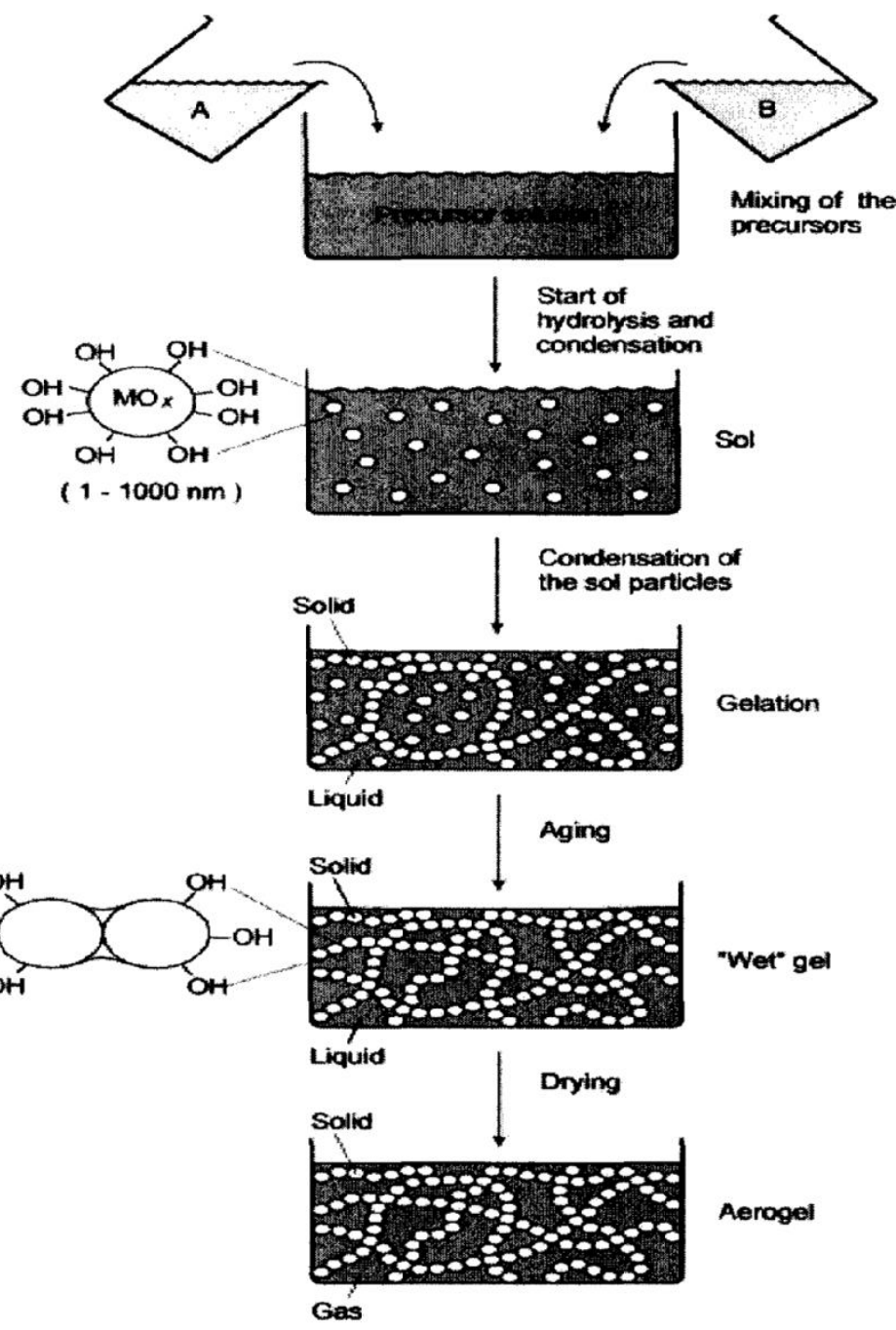

Figure 2.1: Fabrication mechanisms of aerogels [30] 


\subsubsection{Hydrolysis and Condensation Reactions}

Silica produced by the sol-gel route starts with hydrolysis of silicon alkoxides. Therefore, we obtain the following reaction:

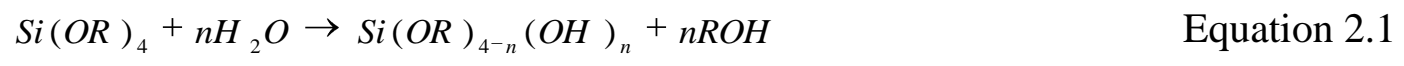

The most important in this reaction is the formation of a metal hydroxide $\operatorname{Si}(\mathrm{OR})_{4-n}(\mathrm{OH})_{n}$.

Water concentration and employed catalysts play a very important role in the hydrolysis and condensation processes. The reaction process between silicon alkoxides and low concentration of water $\left(\mathrm{H}_{2} \mathrm{O} / \mathrm{Si} \prec 2\right)$ leads to the formation of partially hydrolyzed monomers. These monomers will then condense and form a completely esterified polymeric species. If the fraction of water increases up to $\mathrm{H}_{2} \mathrm{O} / \mathrm{Si}=4-10$, then the polymeric strands will be completely hydrolyzed in acid catalyst gels. In a base catalyzed systems, a fully polymer is formed while some unhydrolized monomers are still present. These monomers will induce the phase separation. This leads to the formation of two different regions. The first is an aqueous region with hydrophilic polymer and the second one is solvent rich regions with unreacted hydrophobic monomer. The separation of the individual polymeric strands occurs when the concentration of water increases to $\mathrm{H}_{2} \mathrm{O} / \mathrm{Si}=25-50$. Consequently, isolated cyclic structures and more dense spherical particles are formed [31].

During the condensation reaction, a metal hydroxide reacts with another metal hydroxide and give a metal oxide substance [32].

$$
\mathrm{X}_{3} \mathrm{SiOH}+\mathrm{HOSiX}{ }_{3} \rightarrow \mathrm{X}_{3} \mathrm{Si}-\mathrm{O}-\mathrm{SiX}_{3}{ }_{3}+\mathrm{H}_{2} \mathrm{O} \quad \text { Equation } 2.2
$$




\subsubsection{Gelation}

In real systems, hydrolysis and condensation proceed concurrently and generate a very complex overall reaction kinetics. These reactions will increase and will also raise the produced oxide substance's molecular weight [33]. While growing, the oxide metal particles will form some links in 3-D network. Gelation takes place when these links between silica sol particles become giant spanning clusters which reach across the containing vessel. Then, the gel reaches a point where the links formed between the silica sol particles span the vessel that contains the sol. That is called the gelpoint [34].

\subsubsection{Aging}

When the sol-gel silicates are aging in solvents, their surface areas and pore structures will change. The silica surface area can be increased by aging the sol-gel silicates in ethanol and then putting them into water. Consequently, the number of Si-O-R groups will increase and cause the depolymerisation of the silica network. The separation of monomeric and oligomeric silica species will also take place. The gels obtained will be opaque [35].

By ageing the gels in water and then washing them with ethanol, the surface area will be decreased due to the complete hydrolysis of surface $\mathrm{Si}-\mathrm{O}-\mathrm{R}$ groups. This gives a formation of new Si-O-Si bonds and gels optically transparent, with smaller pore sizes and a broader pore size distribution. Higher temperatures, hydroxide and fluoride ions increase the rate of ageing as well as the development of pore size and pore distribution [36]. 


\subsubsection{Drying}

The drying process can be divided into 4 phases: the constant rate period, the critical point, the first failing rate period and the second failing rate period [37].

The Constant Rate Period starts when the gel sample is still very flexible and compliant and ends when the gel becomes rigid. During this phase, the gel becomes smaller in the same ratio as the evaporated water. If the processes of cross-linking and syneresis take place at a high velocity, the gel can shrink faster than the water can evaporate. The Constant Rate Period will stop when the excess water produced during the syneresis phase has completely evaporated .

While the gel dries and shrinks, its structure becomes more compact and its stiffness increases due to some extra cross-linking reactions. The gel sample reaches its critical point when it becomes stiff enough to resist shrinkage. At the critical point, the water starts moving back from the gel pores it was covering. Very large pressures are produced across the interfaces of the liquid in the pores because of the small size of the gel pores and the presence of surface tensions.

Although, the liquid recedes into the porous structure of the gel, a thin liquid film remains on the pore walls. Flow in the thin liquid film, which is followed by the evaporation and the evaporation from the filled pore area generate a further drying process. When the gel sample dries to the point where its lower surface is in contact with the container, evaporation will be greater from the upper face. The lower surface of the sample will have filled pores and the upper surface empty pores. When the upper surface pores begin to empty, the capillary stress increases. Thus, at any stage in this phase, cracking may occur. 
It is more difficult for the thin liquid film to stay on the pore wall because of evaporation. During the Second Falling-Rate Period, the thin liquid film on the pore wall will be broken. The lower region of the gel sample with filled pores needs to be evaporating so that the liquid reaches the surface. It is very difficult to predict the drying rate during this phase because it will depend on the pore size distribution, the surface of the gel sample, the presence of liquid at irregular pore surfaces (surfaces between the main liquid interface and the outer gel interface) and the relative temperature of the bulk .

\subsubsection{Supercritical Drying}

Drying of the aerogel samples leads to cracks. Crack-free aerogels can be obtained by applying the supercritical drying method. This involves putting the solvent into a supercritical state, which will lead to the disappearance of liquid/gas interfaces in the pores [38].

Supercritical drying is performed by placing the gel inside an autoclave and slowly raising its temperature, which will also increase its pressure. When the temperature and pressure of the gel are above the critical point of the solvent, the fluid is slowly vented at constant temperature, while the pressure is decreased. The cooling process of the autoclave starts when its pressure is similar to the surrounding air and ends when it reaches equal ambient temperature .An alternative way to perform supercritical drying, while avoiding heating/cooling of the autoclave, is to exchange the solvent with supercritical $\mathrm{CO}_{2}$. Two major requirements have to be met:

- Time-consuming solvent exchange before the drying process

- The miscibility of pore liquids with $\mathrm{CO}_{2}$ 
To avoid evaporation of the solvent, in supercritical drying, nitrogen is used to prepressurize the vessel [39].

Aerogels can also be prepared using the so-called "fast supercritical extraction technique". This fabrication process involves two majors steps which occur inside a mold shown in figure 2.2 .

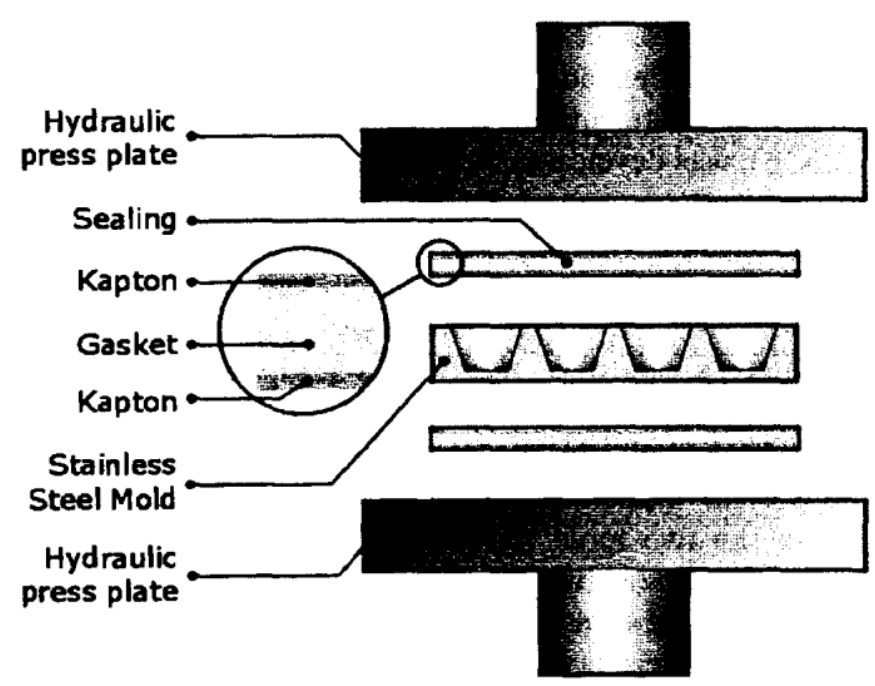

Figure 2.2: Schematic of the mold and press configuration for aerogel processing [40]

The first step was the formation of the sol-gel when the silica was polymerized and the second was the extraction of the water and alcohol solutions. The mold, which contained a mixture of silicon alkoxide precursors of aerogel, was placed in the hydraulic press with $75 \mathrm{KN}$ force acting on it. Then, the mold was heated from ambient to a supercritical temperature $\left(\sim 265-300^{\circ} \mathrm{C}\right)$ by a temperature ramp of $1.25^{\circ} \mathrm{C} \mathrm{min}^{-1}$. As the mold temperature increased, the pressure rose to $21 \mathrm{MPa}$. At this point, the evacuation of some gases occurred and the pressure reduced to $13 \mathrm{MPa}$. After 3.25 hours, the load was dropped to $4 \mathrm{KN}$, the supercritical gases were evacuated and the internal mold pressure decreased until its value reached the ambient pressure with a high temperature. Then, the 
press was cooled down to the ambient temperature, which took 1.5 hours, allowing the retraction of the platens of the hydraulic press and the collection of resulting aerogels.

\subsection{Flexible aerogel blanket fabrication}

The aerogel matrix of a blanket can be prepared from organic materials, inorganic materials and a mixture of the two. Suitable materials for forming organic aerogels are polyacrylates, polystyrenes, phenol furfuryl and polyfurfural alcohol, melamine and phenol formaldehydes .These materials can be damaged by heat at high temperatures, so organic aerogel blankets are not suitable for many uses [41].

The inorganic aerogels exhibit better qualities for aerogel blanket invention. Inorganic aerogels can be made from silicon, aluminum, titanium and vanadium. To obtain an inorganic aerogel, a metal alkoxide, preferably containing around 1-6 carbon atoms in each alkyl group, needs to put through the hydrolysis and the condensation processes. Some examples of appropriate metal alkoxides used in inorganic aerogels are TEOS, TMOS, zirconium, aluminum and titanium isopropoxides. These metal alkoxides can be divided in two groups based on temperature applications. For high temperatures applications, the refractory metal alkoxides such as alumina, titania, zirconia and yttria are used, while at low temperatures, the non-refractory metals (iron, nickel, cobalt) are more suitable $[42,13]$. To improve the Infrared Radiation (IR) opacification of aerogel blankets, mixtures of non- refractory and refractory metals such as silicon can be used [43]. Many factors including the type of metal alkoxides, the solution $\mathrm{pH}$, and the ratio between alkoxide/alcohol/water will directly affect the formation process of inorganic aerogel blankets [44]. 
Figure 2.3 outlines the fabrication process of an aerogel blanket. After mixing a $1300 \mathrm{~mL}$ of pre-hydrolyzed silica precursor with $1700 \mathrm{~mL}$ of alcohol and stirring the solution for 15 minutes, a solution of High Formamide (HF) is added slowly until gelation occurs. The resulting solution is poured on a blanket placed in a container. The mixture is then aged for a sufficiently long period in a sealed bath of ethanol at $50^{\circ} \mathrm{C}$. The alcohol inside the gel is removed by using a subcritical and supercritical $\mathrm{CO}_{2}$ extraction technologies over 4 days.
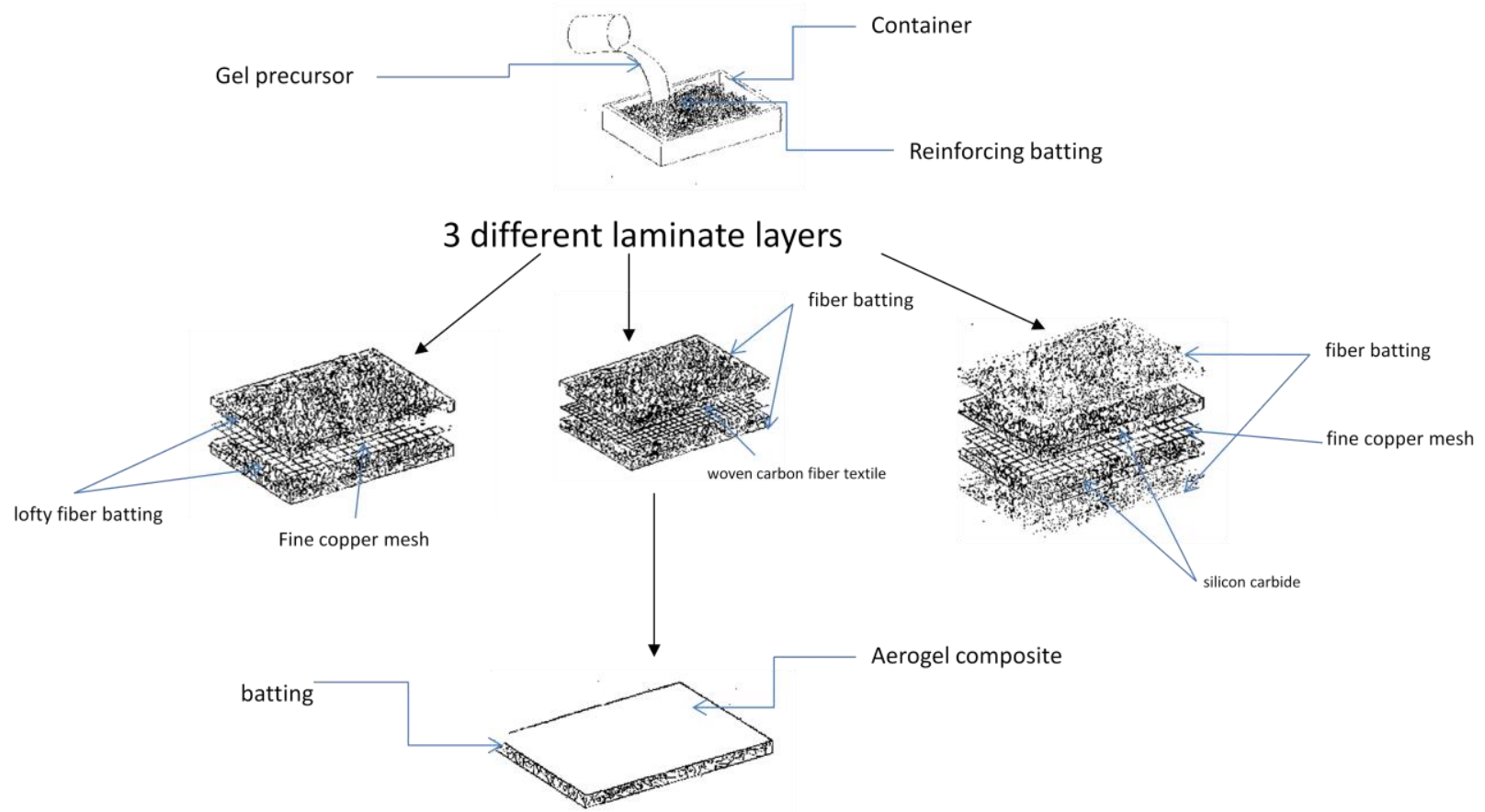

Figure 2.3: General fabrication process of a flexible aerogel blanket [45]

The aerogel blankets can be made of lofty silica or 3, 4 or 5 layer laminate or polyester or silica. Then, the chemical composition and the geometry of the aerogel composite materials will affect the thermal and the mechanical properties of the blanket. 
Another way to produce flexible aerogel blanket is by using water glass precursor and glass wool modified by alumina sol via ambient drying. The flow chart of this technique used for the preparation of aerogel blanket is shown in figure 2.4:

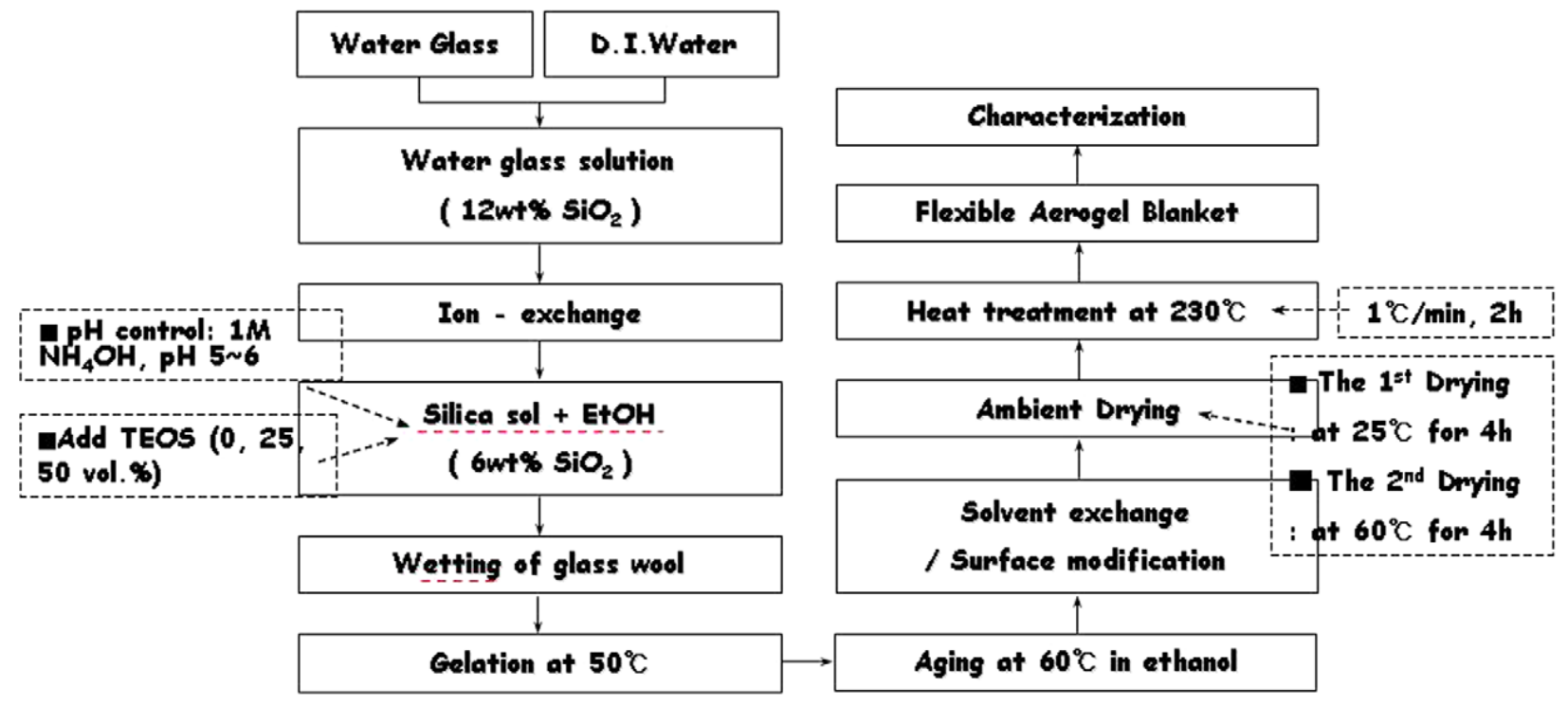

Figure 2.4: Flowchart of the preparation of flexible aerogel blanket [46]

As the result of the dilution of $100 \mathrm{~g}$ of water glass in $150 \mathrm{ml}$ of deionised water, a water glass solution was obtained. The water glass solution passed through the amberite resin inside the ion exchange column drop by drop using micro motor. At the bottom of the glass column, a silica sol with a $\mathrm{pH}$ in the range 2.3 was collected and then, mixed in a volume ratio 1:1 with ethanol. Ammonium hydroxide and tetra ethoxy silane (TEOS) solutions were added to the silica ethanol mixture. The wetted glass wool, obtained by wetting glass wool with the silica ethanol TEOS mixture, was placed in oven for gelation at $50{ }^{\circ} \mathrm{C}$ for 3 hours. After being soaked in $200 \mathrm{ml}$ of ethanol solution, the wet gel blanket was aged at room temperature for 48 hours. The aging process will continue for an additional 48 hours when the solvent exchange solution (167 $\mathrm{ml}$ of $\mathrm{n}$-hexane, $17 \mathrm{ml}$ of 
tetra methyl chloro silane) and $10 \mathrm{ml}$ of isopropyl alcohol were added to the wet gel

blanket. After been dried for 28 hours, the gel blanket is heat treated at $230^{\circ} \mathrm{C}$ at a rate of $1{ }^{\circ} \mathrm{C} / \mathrm{min}$ for 2 hours to obtain silica aerogel blanket.

\subsection{Mechanical properties of a flexible aerogel blanket}

\subsubsection{Mechanical properties of aerogel}

Aerogel is an interesting porous material because of its unique properties, which give it a wide range of uses. Unfortunately, aerogels are very compliant with typical moduli on the order of MPa [47]. Young's modulus of aerogel was measured by Gronauer et al., [48] using sound velocity technique and Woignier and Phalippou [49] using three point bending tests. Arvidson and Scull [50] measured compressive properties of silica aerogel at $20 \mathrm{~K}, 76 \mathrm{~K}$ and $295 \mathrm{~K}$.

LeMay [51] and Pekala [52] indicated that aerogel morphology can be affected by the variation of the $\mathrm{pH}$ and the density of the precursor gel (alcogel). They also used a scaling law to describe the behavior of the compressive modulus and compared the compressive moduli of silica aerogel to that of organic materials.

The mechanical behavior of fiber-reinforced and unreinforced silica aerogel was studied using compression tests at ambient temperature. Figure 2.5 exhibits the stressstrain curves for various aerogel samples under compressive loading. The aerogel samples tested have different densities and different fiber contents. For a given target density, the compressive strength decreases when the values of fiber percentage increase, while the strain at fracture does not follow a perceptible trend. When the fiber percentage is the considered parameter, the strain at fracture decreases with increases in target densities, while the compressive strength increases. 


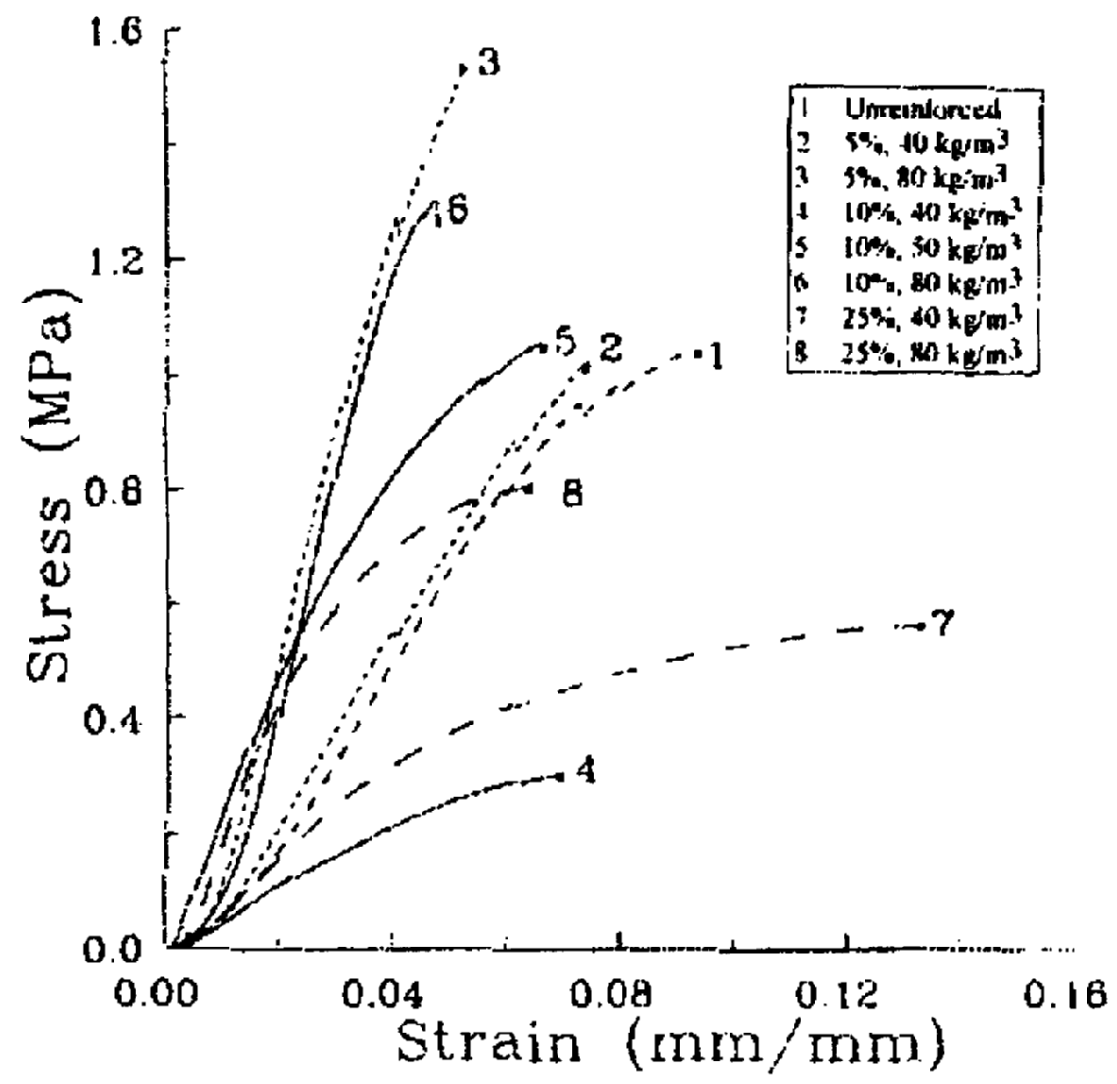

Figure 2.5: Representative stress-strain curves for aerogels under compressive loading. Degree of fiber reinforcement (mass \%) and target density are shown in the legend [53]

Moner-Girona et al. [54] also investigated the mechanical properties of silica aerogels by doing some microindentation measurements on a Nanotest 550 Indenter.

\subsubsection{Mechanical degradation of aerogel}

Although the supercritical drying method allows the elimination of capillary stresses during the preparation of aerogel, some tension stresses remain. When aerogel samples are exposed to high temperature, the very low permeability of the liquid expansion in the pores will create some stresses. Other stresses take place during the depressurization process because of the change in fluid compressibility. Tensions stresses 
generate the cracking of the skeleton. Tension stresses are related to the fluid flow speed out of the gel network. This lead to the swelling and the breaking of aerogels [55-58].

To study the development of the crack velocity according to the stress intensity factor, the chemical susceptibility factor n, a corrosion factor was introduced. The chemical susceptibility factor $\mathrm{n}$ can be calculated by using the slope of the logarithmic curve of strength, $\sigma_{R}$ versus the stress rate $\sigma$. The mathematical relation between the strength or modulus of rupture and the stress rate is expressed by:

$\sigma_{R}=C \dot{\sigma}^{\frac{1}{n+1}}$ Equation 2.3

The modulus of rupture of aerogel samples $\sigma_{R}$ is defined as:

$$
\sigma_{R}=\frac{8 F^{\bullet} L}{\pi_{D^{3}}}
$$

Equation 2.4

The stress rate $\sigma$ is obtained using the relationship:

$$
\dot{\sigma}=\frac{6 E D}{L^{2}} y
$$

An experiment with 15 aerogel samples was performed in order to determine the chemical susceptibility factor $\mathrm{n}$. Five different stress rates with crosshead speeds ranging from 0.01 to $50 \mathrm{~mm} / \mathrm{min}$ were used. The results of this experiment are shown in figure 2.6 (a). All samples have the same bulk density and the same Young's modulus E value of $13 \pm 2 \mathrm{MPa}$. The straight line corresponds to the different values of the chemical susceptibility factor $\mathrm{n}$ using a linear least square method. Aerogel samples can submit the maximum stress $\sigma_{c o}$ at the end of the depressurization process. During the depressurization process, the pressure drops from $13 \mathrm{MPa}$ to 0.1MPA and some aerogel samples can be broken. Figure 2.6 (b) shows the maximum stress as a function of the 
stress rate.

There are still uncertainties as to the value of stress for which the facture of aerogel

occurs. The supercritical drying during the depressurization can induce aerogel fracture.

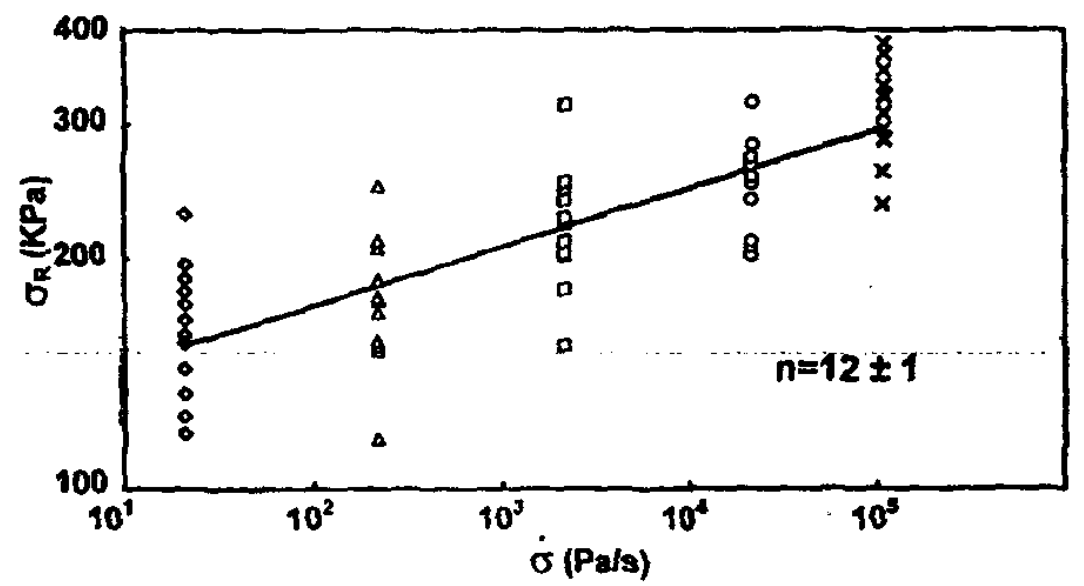

(a)

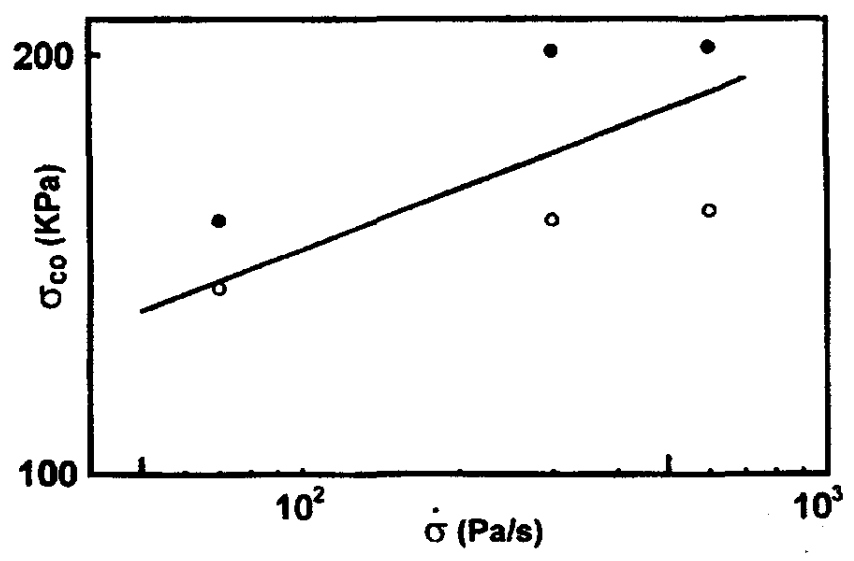

(b)

Filled circles correspond to broken aerogels Open circles correspond to save aerogels

Figures 2.6: Logarithmic plot of the stress versus the stress rate [59] 


\subsubsection{Mechanical behavior of an aerogel blanket}

There are relatively few experiments related to the mechanical properties of flexible aerogel blankets. The compressive strain under hydrostatic pressure of aerogel blanket "Spaceloft" (product line) was compared to that of a newly developed prototype aerogel blanket. This experiment started with the encapsulation of an aerogel blanket in a Mylar bag. The Mylar bag was placed between two flat plates, sealed and heated at ambient pressure. Later, the Mylar bag was placed in the Amter Thermal meter within the hyperbaric chamber as shown in figure 2.7.

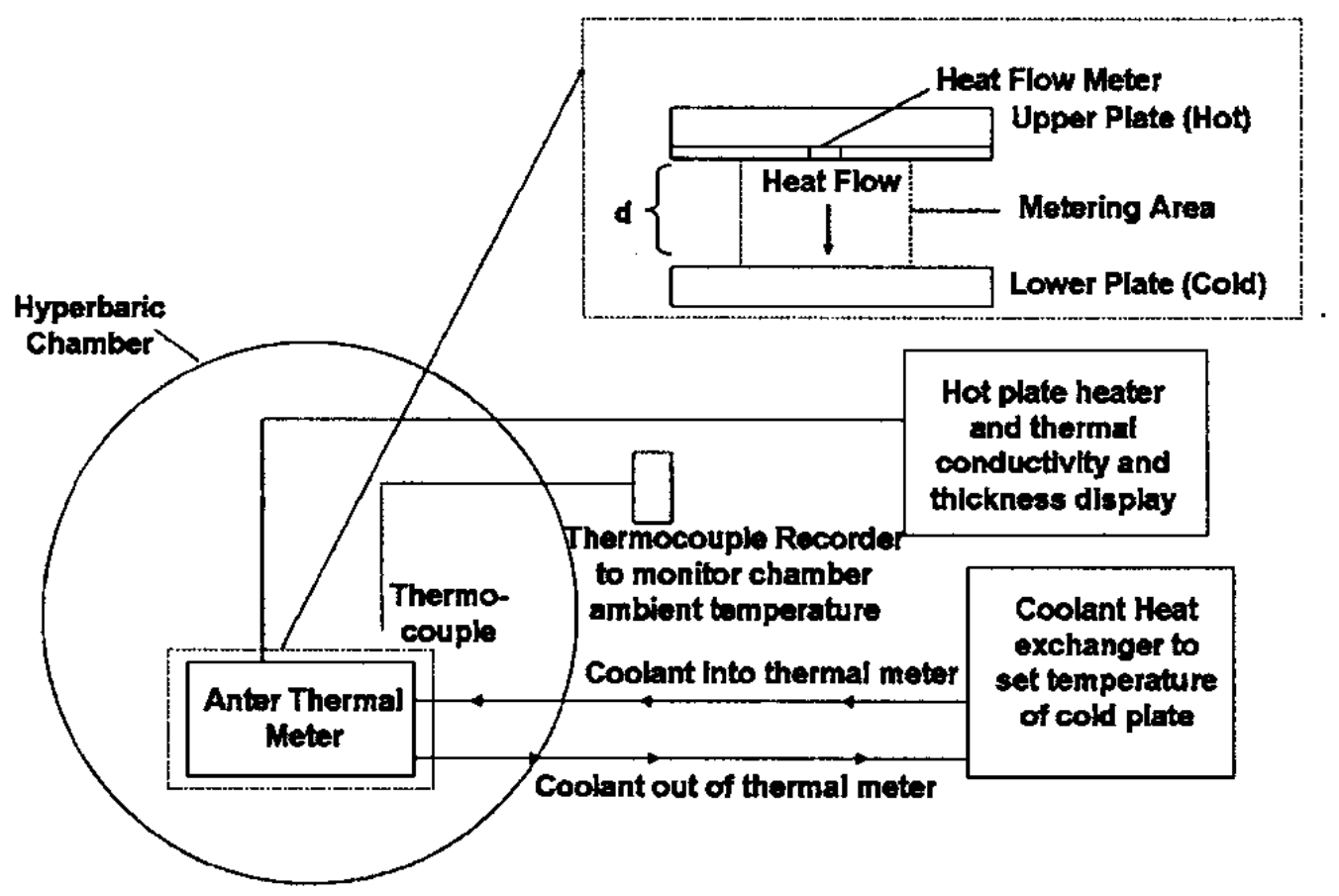

Figure 2.7: Schematic of experimental set up [60]

For different values of the pressure, compression/decompression cycles were performed.

The curves compressive strain-hydrostatic pressure for both aerogel blankets are plotted in figure 2.8. 


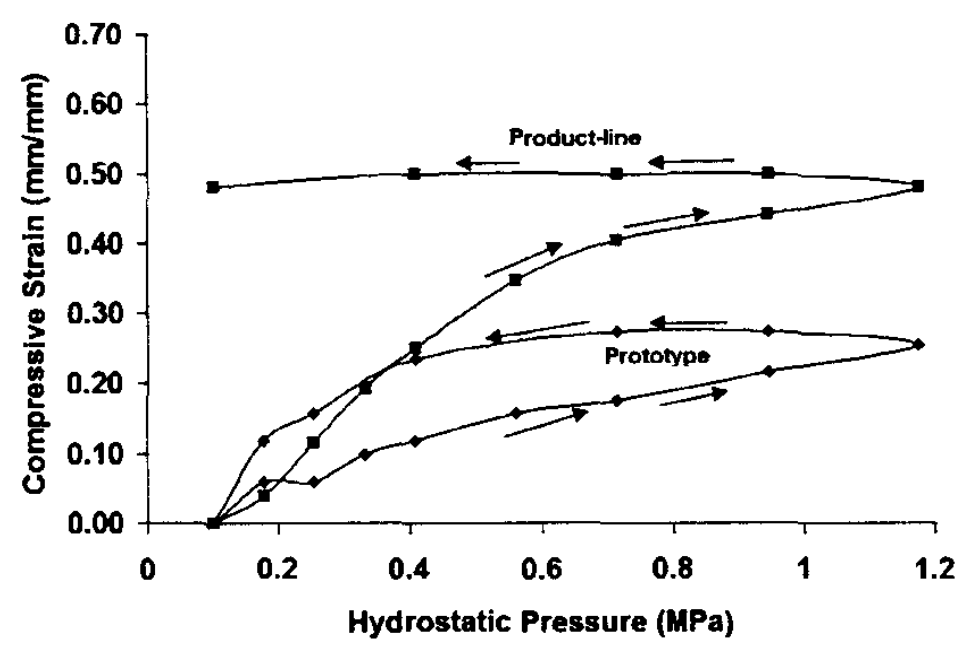

Arrows indicates the direction change during the experiment

Figure 2.8: Variation of the compressive strain of aerogel blanket samples with hydrostatic pressure [60]

The maximum compressive strains of the Spaceloft aerogel blanket and the prototype blanket were $0.48 \mathrm{~mm} / \mathrm{mm}$ and $0.25 \mathrm{~mm} / \mathrm{mm}$ respectively under hydrostatic pressure 1.2 $\mathrm{MPa}$. During the compression phase, the values of compressive strain of the prototype aerogel blanket were reduced to $50-100 \%$ of its values in the decompression phase. During the decompression phase, the strain of the product line aerogel blanket remained constant, while the strain of the prototype aerogel blanket returned to its initial thickness.

\subsection{Thermal properties of flexible aerogel blanket}

\subsubsection{Thermal Conductivity of aerogel}

The heat is transferred through insulating materials in three possible ways: conduction (solid and gas), convection and radiation [61-63]. Consequently, the total effective conductivity will be the sum of conductivity for the 3 modes of heat transfer: 


$$
k_{\text {total }}=k_{g}+k_{s}+k_{r}+k_{c}
$$

At ambient temperature and pressure, the convective thermal heat transfer component $k_{c}$ can be neglected because the aerogel materials have extremely small pores. The mean pore diameter of aerogel is approximately $20 \mathrm{~nm}$ [64].Then, the total effective conductivity for aerogel materials will be:

$$
k_{\text {total }}=k_{g}+k_{s}+k_{r}
$$

Equation 2.7

The total thermal conductivity of opacified monolithic aerogel was measured at different temperatures and densities. The results of this dependence are shown in figure 2.9.

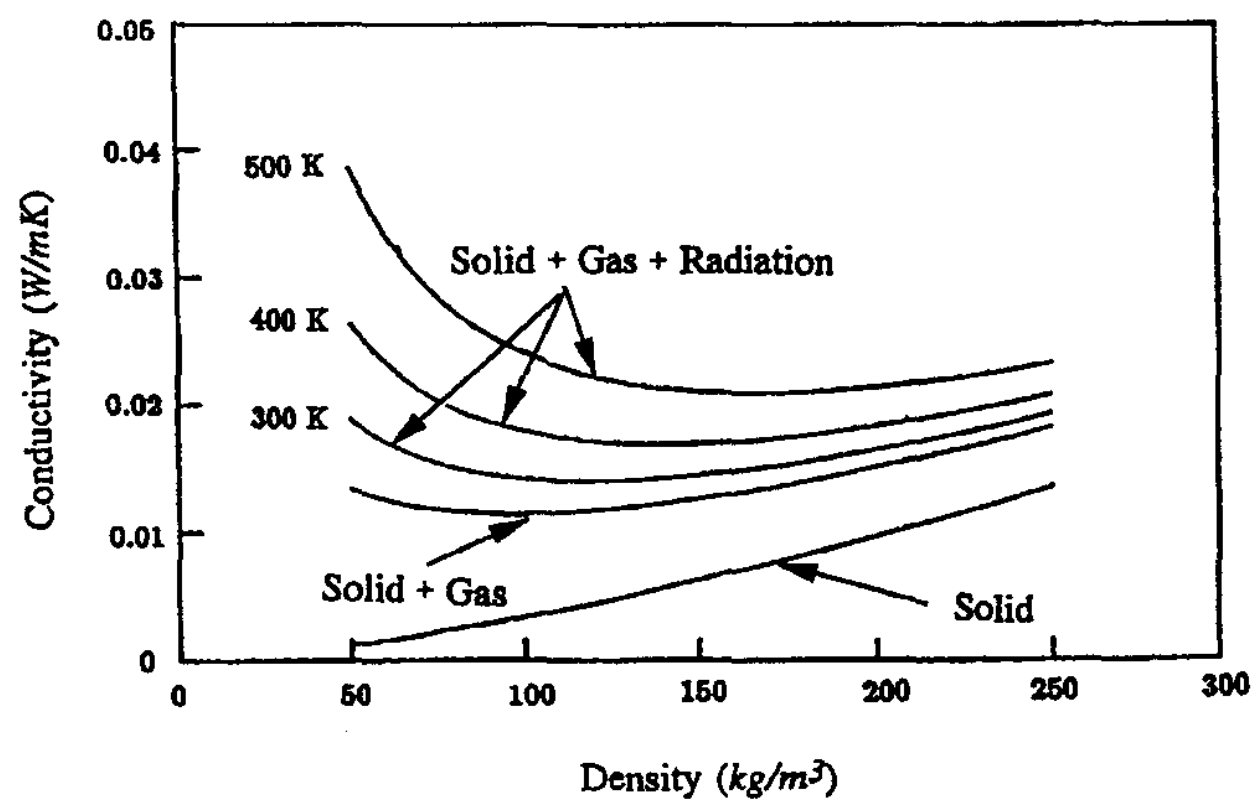

Figure 2.9: Variation of Thermal Conductivity with density at different temperatures [65]

The variation of the total conductivity with densities can be minimized because the radiative and gaseous conductivities decrease with increasing density, while 
the solid conductivity increases. The gaseous and radiative conductivities are larger than the total conductivity for low densities aerogels. The solid conductivity becomes increasingly dominant when the density increases. The minimum thermal conductivity at $300 \mathrm{~K}$ is $\mathrm{k}=0.014 \mathrm{~W} / \mathrm{mK}$ and occurs at a density approximately equal to $120 \mathrm{~kg} / \mathrm{m}^{3}$.

The total thermal conductivities measurements of some aerogels samples were performed for mean temperatures between 10 and $275 \mathrm{~K}$, with temperature differences from 6 to $16 \mathrm{~K}$ and gas pressure below $10^{-3} \mathrm{~Pa}$. These aerogels samples, mixtures of aerogel silica powders and black carbon powders are shown in table 2.1. The measurements of aerogels samples were performed with external loads of $1.5 \times 10^{4} \mathrm{~Pa}$ and $10^{5} \mathrm{~Pa}$.

Table 2.1 Characteristics of aerogel samples measured [66]

\begin{tabular}{|l|r|l|l|r|}
\hline & $\begin{array}{l}\text { Amount } \\
\text { of silica } \\
\text { aerogel } \\
\text { (wt \%) }\end{array}$ & $\begin{array}{l}\text { Amount } \\
\text { of } \\
\text { carbon } \\
\text { black } \\
\text { (wt \%) }\end{array}$ & $\begin{array}{l}\text { Aerogel } \\
\text { grain } \\
\text { density } \\
\left(\mathrm{kg} / \mathrm{m}^{3}\right)\end{array}$ & $\begin{array}{l}\text { Powder } \\
\text { density } \\
\left(\mathrm{kg} / \mathrm{m}^{3}\right)\end{array}$ \\
\hline namples 1 & 100 & 0 & 150 & 150 \\
\hline number 1 & 95 & 5 & 150 & 140 \\
\hline number 3 & 90 & 10 & 150 & 125 \\
\hline number 4 & 85 & 15 & 150 & $120-125$ \\
\hline number 5 & 90 & 10 & 210 & $118-123$ \\
\hline
\end{tabular}

The results of the total thermal conductivity measurements are given in figure 2.10. In both cases, sample 1 , with $100 \%$ of silica aerogels, has the highest total thermal conductivity. By adding some carbon black material to aerogel, the total thermal conductivity is reduced. Sample 5, with $10 \%$ of carbon black and least powder density, possesses the lowest total thermal conductivity rating. The error variation in the measured total thermal conductivity is approximately $10 \%$. These errors arise from the uncertainty in sample thickness and the inconsistency of the electrical heating power. 
Errors in electrical heating power are less than $2 \%$ and the uncertainty in the measurement of samples thickness is $\pm 10 \%$.
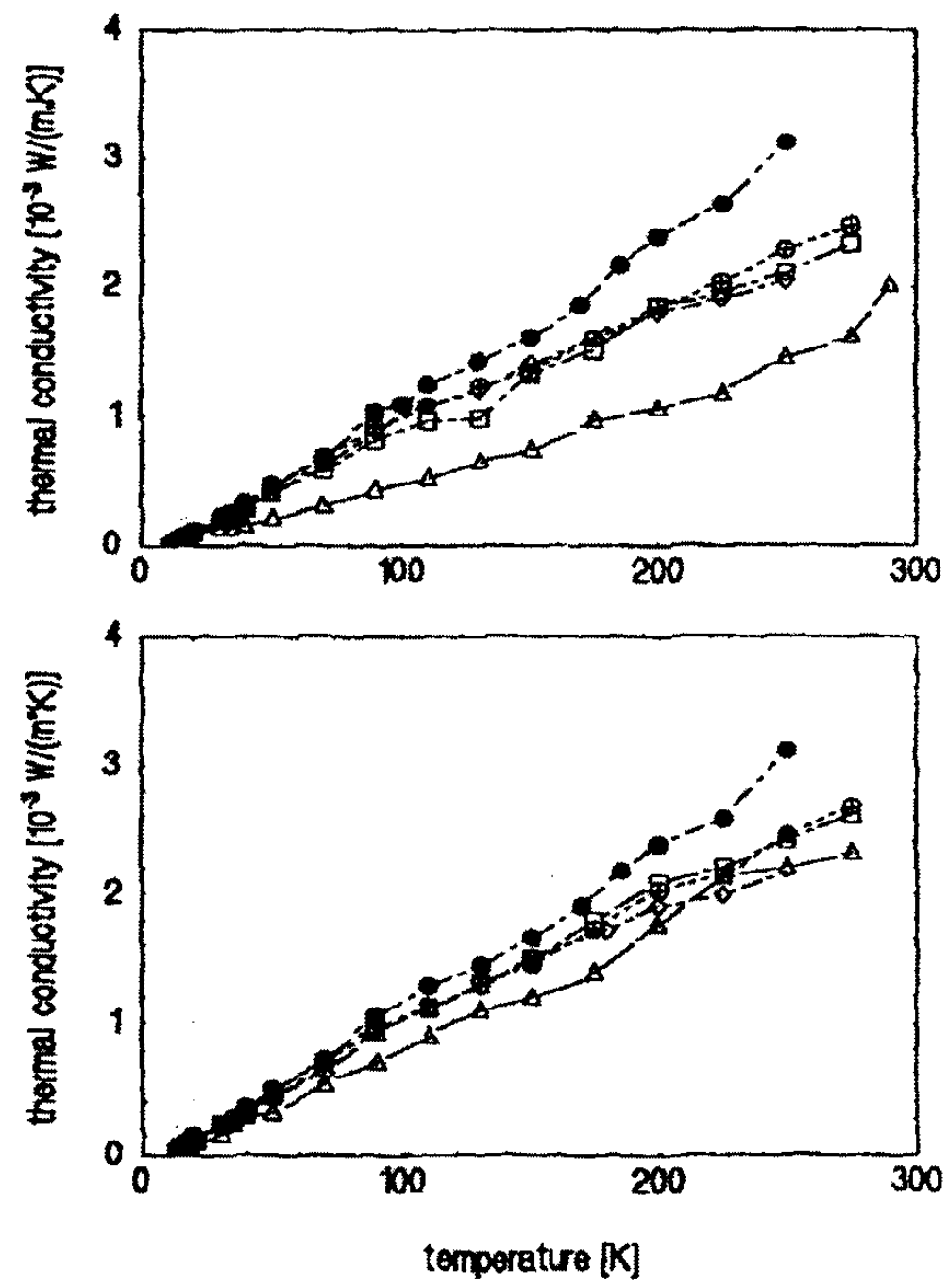

Figure 2.10: Total Thermal Conductivity of Aerogel Powder Samples versus Temperature for an External Load of $1.5 \times 10^{4} \mathrm{~Pa}$ (top) and $10^{5} \mathrm{~Pa}$ (bottom): • ,\#1, $\oplus, \# 2, \square, \# 3, \diamond \# 4, \Delta, \# 5$. [66]

\subsubsection{Thermal Conductivity of an aerogel blanket}

Aerogel blankets are fabricated using a gel after a supercritical drying process and a lofty reinforcement which is infiltrated [25, 45]. The reinforcing systems of aerogel 
composites can be changed by preparing different material of fibers. To study the thermal performance of some insulating materials, five aerogel blankets samples were prepared. Table 2.2 summarizes the chemical composition of those samples. In general, the samples were prepared using lofty polyester or quartz wool from fiber, carbon black dopant and carbon microfiber. In the samples B, C, D and E, the dispersion agent Disperbyk 184 was used.

Table 2.2 Chemical composition of some aerogel blanket samples [45]

\begin{tabular}{|c|c|c|c|c|c|}
\hline Samples & $A$ & $B$ & C & D & $E$ \\
\hline $\begin{array}{l}\text { reinforcing } \\
\text { systems }\end{array}$ & $\begin{array}{l}\prec 2 \\
\text { denier } \\
\text { lofty } \\
\text { polyester }\end{array}$ & $\begin{array}{l}\text { Quartz } \\
\text { wool } \\
\text { from } \\
9 \mu_{m} \\
\text { fibers }\end{array}$ & $\begin{array}{l}\text { Quartz } \\
\text { wool from } \\
9 \mu_{m} \\
\text { fibers + } \\
5 \% \\
\text { carbon } \\
\text { black } \\
\text { dopant } \\
+ \\
3 \% \\
\text { carbon } \\
\text { microfiber }\end{array}$ & $\begin{array}{l}\text { Quartz } \\
\text { wool from } \\
9 \mu_{m} \\
\text { fibers } \\
+ \\
6 \% \\
\text { carbon } \\
\text { black } \\
\text { dopant } \\
+ \\
4 \% \\
\text { carbon } \\
\text { microfiber }\end{array}$ & $\begin{array}{l}\text { Quartz wool from } \\
9 \mu_{m} \text { fibers } \\
+ \\
6 \% \text { carbon black } \\
\text { dopant } \\
+ \\
4 \% \text { carbon } \\
\text { microfiber } \\
+ \\
10 \% \\
\text { polydimethylsilo- } \\
\text { xane dopant }\end{array}$ \\
\hline
\end{tabular}

The carbon microfibers, used in the preparation of these aerogel blankets, have a diameter of 0.1 to $100 \mu_{m}$ and a length of 1 to $2 \mathrm{~mm}$. The measurement of thermal conductivity of these flexible aerogel blankets was performed with the guarded hotplate according to the standard ASTM C-177. Figure 2.11 shows the variation of thermal performance according to the temperature for the samples describes in table 2.2. 


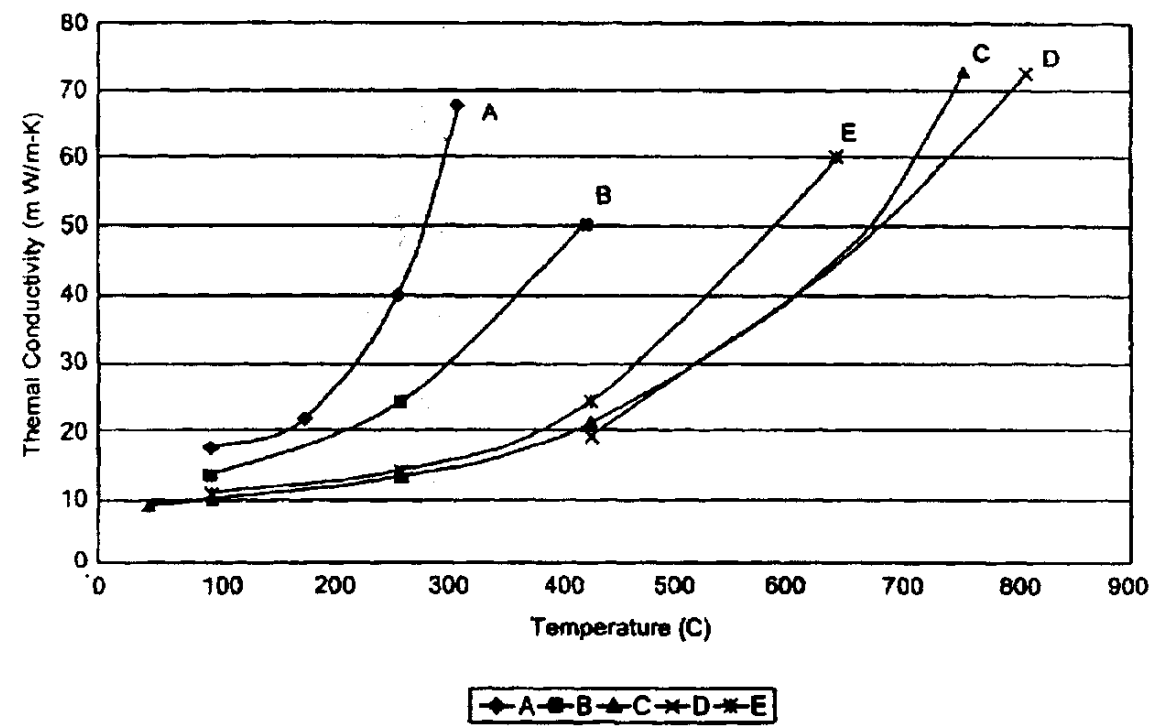

Figure 2.11: Thermal performance of some aerogel blankets samples [45]

Sample A had temperature variations from 100 to 300 degrees and had the highest thermal conductivity. To reduce the thermal conductivity of the blankets, samples B, C, $\mathrm{D}$ and $\mathrm{E}$ were prepared using quartz wool made of $9 \mu_{m}$ fibers with density of $0.005 \mathrm{~g} / \mathrm{cc}$, dried composite of black carbon dopant and carbon microfiber. Sample D had the biggest range of temperature variation (from 50 to 800 degrees) and the lowest thermal conductivity because it contained the largest amount of carbon black dopant and the largest amount of carbon microfiber. One should note that the addition of the polydimethylsiloxane dopant increased the thermal conductivity of sample E beyond that of C and D.

\subsection{Computer simulation of aerogel}

\subsubsection{Computer modeling of mechanical structure property relationship of aerogels}

During the research on aerogels' properties, computer simulation measured the connectivity of the aerogel network in a space. The connectivity of the aerogel network is 
a very important factor for the stiffness of an aerogel. Three computer models were developed: a diffusion-limited aggregation (DLA), a cluster-cluster aggregation (CCA) and a diffusion-limited cluster-cluster aggregation (DLCA). These aggregation models uses cubic lattices and periodic boundary condition and generate a constant number of diffusing particles from random locations on the lattice. DLA models simulate many clusters that grow and randomly walk together from a distant circle or sphere to generate the aggregation process of these particles. In the CCA model, all clusters diffused and grew by sticking at their first contact. Each cluster moved one lattice space in a random direction at each time step [67-69].

Computer simulation of aerogels was useful in understanding the physics behind the scaling relationship between the modulus and the density [70]:

$$
E \sim \rho^{m}
$$

The relative modulus values of aerogel predicted were different from the ones obtained from the DLCA model and the open-foam model proposed by Gibson and Ashby [71]. The main reason for this deviation was the presence of a huge quantity of clusters connected to the backbone of the aerogel network at a point known as "deadends".

A new algorithm called "dangling bond deflection" with DLCA was combined to generate aerogel models with extensive loop structure and negligible dead-ends. The finite element method was used to measure the bulk moduli of the resulting model. The bulk moduli of the aerogel model fell perfectly on a power fit with $\mathrm{m}=3.6$. During the gelation process, the rate of dangling bond deflection was varied but the values of the bulk moduli at the same density and the scaling exponent remained almost 
constant. The bulk moduli of the resulting models were calculated by the finite element method using the strain energy $\varepsilon_{s}$ and the volumetric strain $\alpha$ :

$$
E=\frac{2 \varepsilon_{s}}{V(1+\alpha) \alpha^{2}}
$$

Figure 2.12 illustrates the cumulative contributions of each bond to the total strain energy of the aerogel network for different models. A small fraction of bonds carried most of the strain energy absorbed by the model. Moreover the density of aerogel decreases with the fraction of energy bearing bonds.

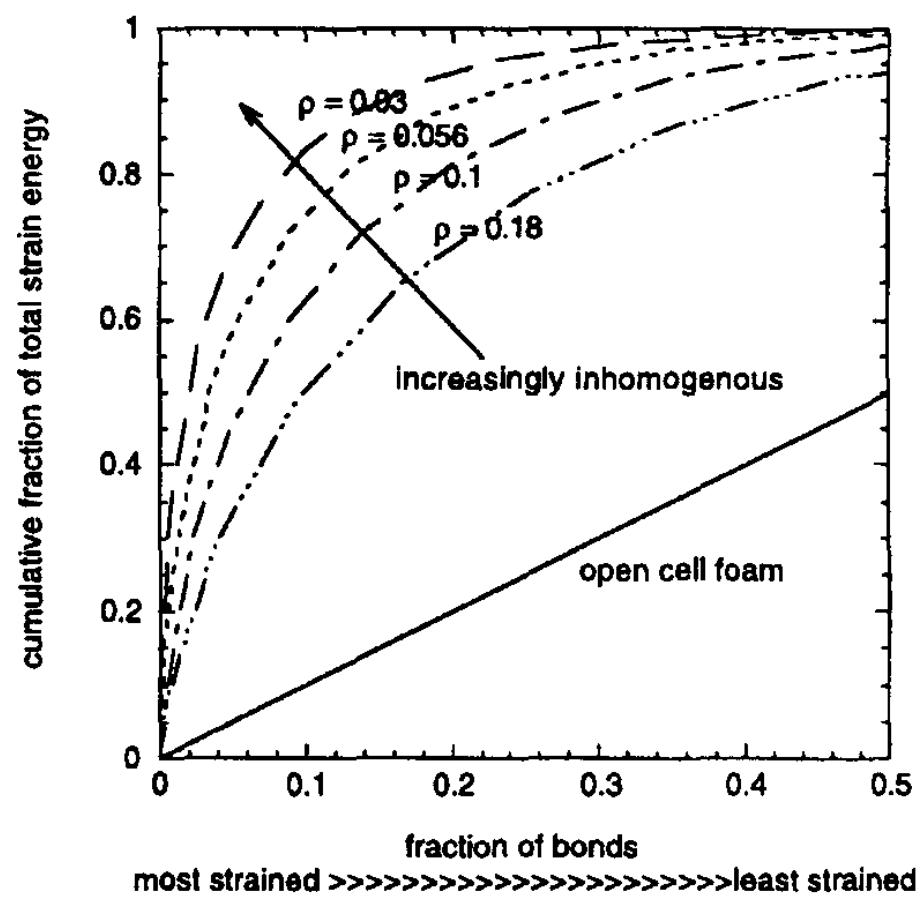

Figure 2.12: Cumulative distribution of strain energy in a bond [72] 


\subsubsection{Finite element analysis of heat transfer in aerogel}

A unit cell model was used to evaluate the heat transfer by conduction inside an aerogel. This model is defined as a single fiber surrounded by a concentric rectangular interstitial void. Three different interstitial voids were used: argon, carbon dioxide and aerogel. In this model, the temperature of the two opposing sides was constant and set to match the temperature of a hot and a cold plate of $273 \mathrm{~K}$ and $243 \mathrm{~K}$ respectively. The remaining sides of the unit cell were insulated. This model was created using SolidWorks software and was imported to COSMOS DesignSTAR software to provide the finite element solution. Three fiber of 6 and 15 denier, as shown in figure 2.13, were evaluated: round fiber with a solid cross section, round fiber with four holes and fiber having a cross section with had deep groves (DG) along the longitudinal axis. 

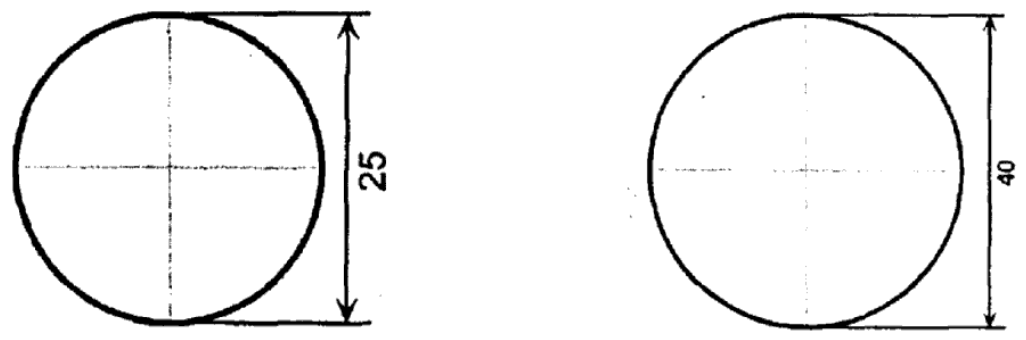

(a)
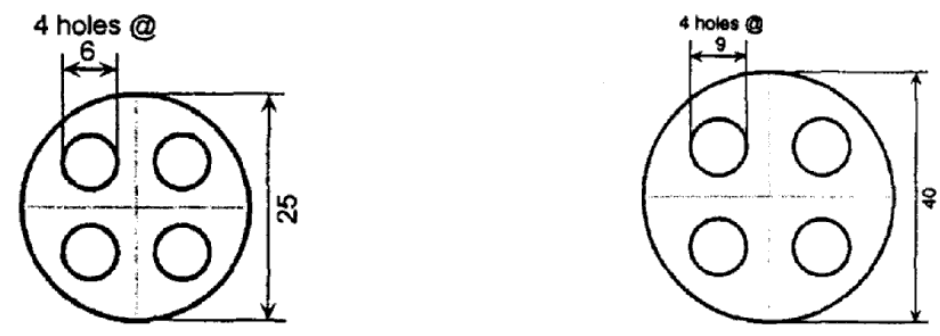

(b)
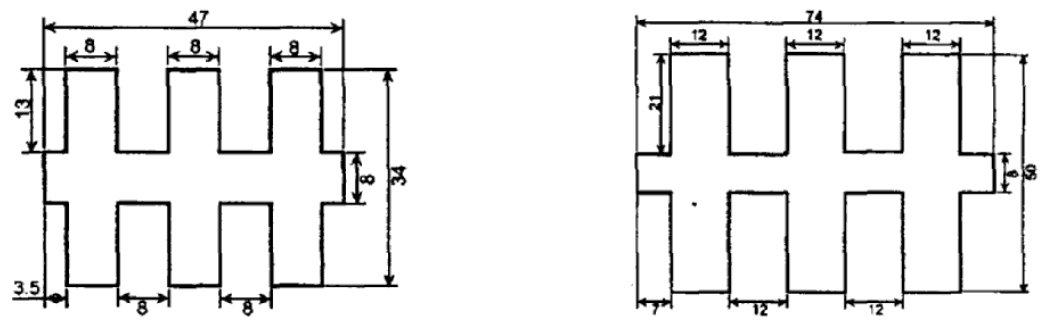

(c)

\section{6 denier is on the left and 15 denier on the right}

Figure 2.13 : Model cross-sectional dimensions of (a) the solid, (b) hollow and (c) 4DG fibers (dimensions in microns) [73].

Effective thermal conductivity was computed for four cases of steady-state conduction at high and low interstitial void fractions for perpendicular and axial orientations of the fiber to the temperature gradient. The results of the effective thermal conductivity for the unit cell insulation models are shown in figure 2.14. 


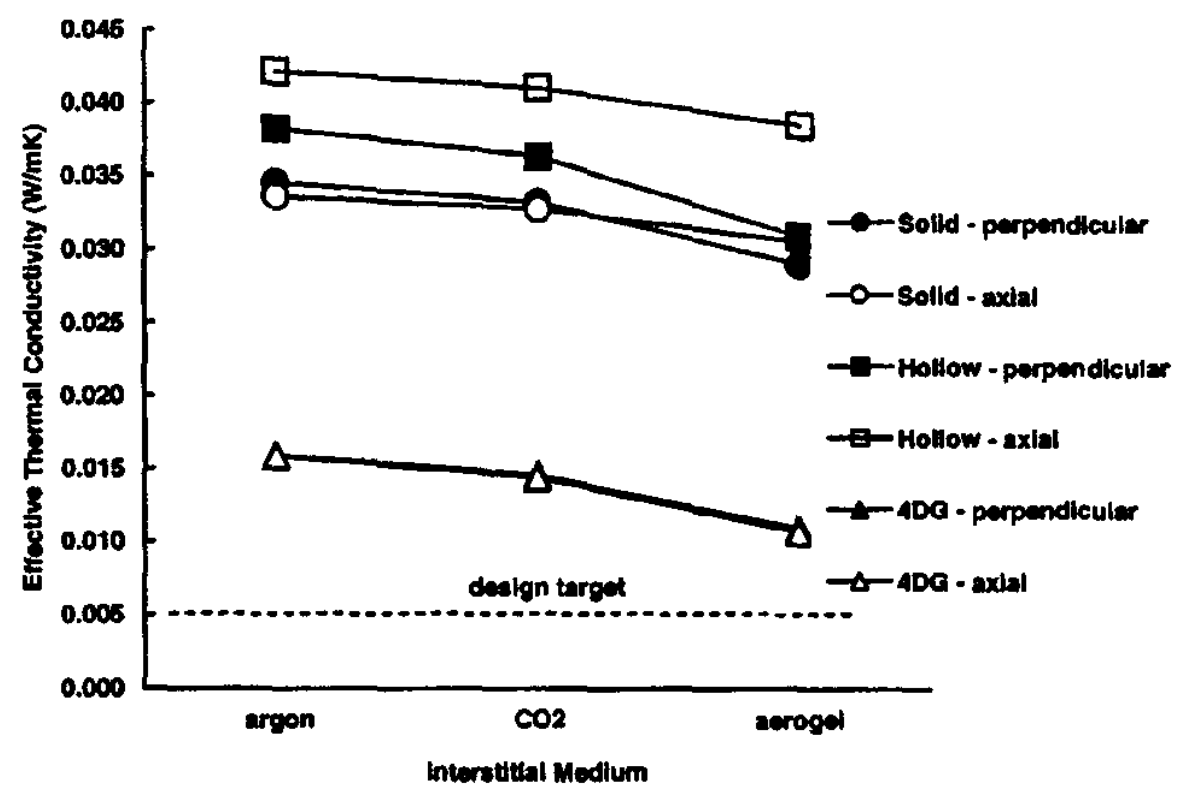

(a)

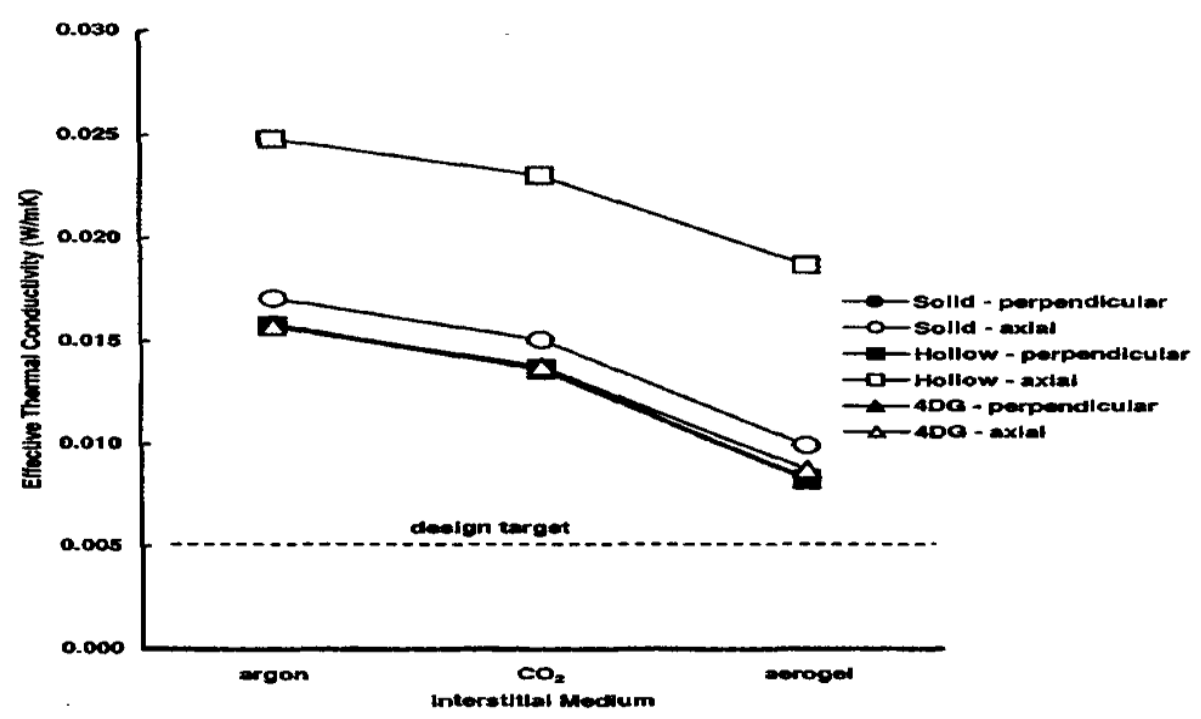

(b)

Figure 2.14: Effective thermal conductivity of insulation materials: (a) low interstitial void fraction (b) high interstitial void fraction [73]

Comparing all the curves, figure 2.13, clearly shows that the fiber orientation to the heat flow is very important. When a fiber is normal to the heat flow, it requires that heat transfer occurs through the interstitial void medium and provides a relatively small conductivity pathway across the unit cell. A fiber aligned axially with the heat flow 
provides a relatively high conductivity pathway across the unit cell and the interstitial void medium. That leads to a reduction of the overall insulating capacity because the interstitial void medium is less conductive than the fiber. The thermal conductivities of all interstitial void media for high void fraction systems are less than low ones. High void fraction systems offer superior insulating properties. At low void fraction, the fiber containing a cross section with deep groves along the longitudinal axis offers more effective overall insulation but it decreases when interstitial void fraction increases. Comparing the thermal performance of the three interstitial void media, aerogels clearly produce a better insulation performance. 


\section{Chapter 3 Experimental Procedures}

\subsection{Description of the Studied Samples}

The flexible aerogel blankets tested in this study were manufactured by ASPEN Aerogels; Inc. Table 3.1 details of some properties of the tested samples.

Table 3.1 Properties of flexible aerogel blanket

\begin{tabular}{|c|c|c|c|c|c|c|}
\hline $\begin{array}{l}\text { Product } \\
\text { name }\end{array}$ & Category & Color & $\begin{array}{l}\text { Silicone } \\
\text { Coated }\end{array}$ & $\begin{array}{c}\text { Nominal } \\
\text { Thickness } \\
{[\mathrm{mm}]}\end{array}$ & $\begin{array}{l}\text { Material } \\
\text { Form } \\
{[\mathrm{mm}]}\end{array}$ & $\begin{array}{l}\text { Maximum } \\
\text { Use } \\
\text { Temperature } \\
\qquad\left[{ }^{\circ} \mathrm{C}\right]\end{array}$ \\
\hline $\begin{array}{l}\text { AR } \\
5400\end{array}$ & PYROGEL & $\begin{array}{l}\text { DARK- } \\
\text { BLUE }\end{array}$ & NO & 6.0 & $150 \times 100$ & 350 \\
\hline $\begin{array}{l}\text { AR } \\
5403 X\end{array}$ & PYROGEL & $\begin{array}{l}\text { DARK- } \\
\text { BLUE }\end{array}$ & $\begin{array}{l}\text { YES } \\
2 \text { Sides }\end{array}$ & 6.0 & $150 \times 100$ & 350 \\
\hline $\begin{array}{l}\text { AR } \\
5401 X\end{array}$ & PYROGEL & $\begin{array}{l}\text { DARK- } \\
\text { BLUE }\end{array}$ & $\begin{array}{c}\text { YES } \\
2 \text { Sides }\end{array}$ & 2.0 & $150 \times 100$ & 350 \\
\hline $\begin{array}{l}\text { AR } \\
5401\end{array}$ & PYROGEL & $\begin{array}{l}\text { DARK- } \\
\text { BLUE }\end{array}$ & NO & 2.0 & $150 \times 100$ & 350 \\
\hline $\begin{array}{l}\text { AR } \\
5223\end{array}$ & PYROGEL & BEIGE & NO & 6.0 & $150 \times 100$ & 650 \\
\hline $\begin{array}{l}\text { AR } \\
3103\end{array}$ & SPACELOFT & WHITE & NO & 6.0 & $150 \times 100$ & 200 \\
\hline $\begin{array}{l}\text { AR } \\
3103 X\end{array}$ & SPACELOFT & WHITE & $\begin{array}{l}\text { YES } \\
2 \text { Sides }\end{array}$ & 6.0 & $150 \times 100$ & 200 \\
\hline
\end{tabular}

Spaceloft and Pyrogel materials, shown in figures 3.1 and 3.2 respectively, are two families of flexible aerogel nanoporous insulation blanket designed for various applications. They are environmentally friendly materials and easy to apply and handle $[74,75]$.

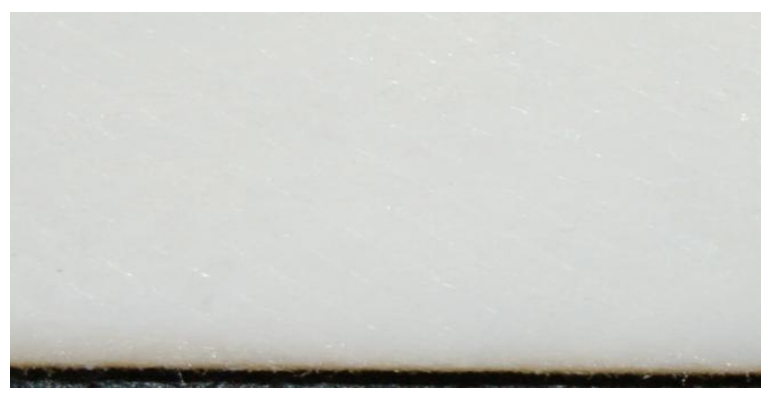

Figure 3.1: Spaceloft AR 3100 series 


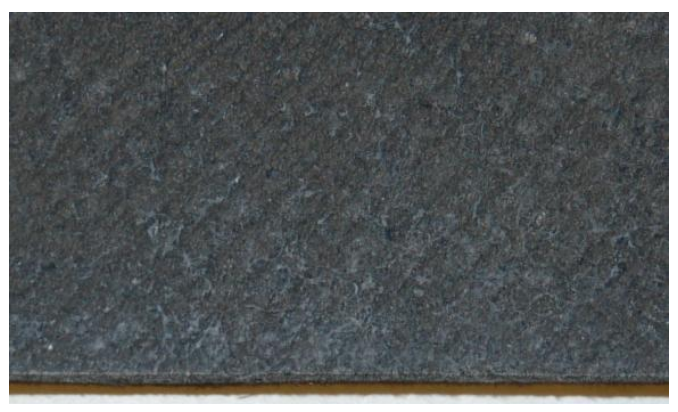

(a)

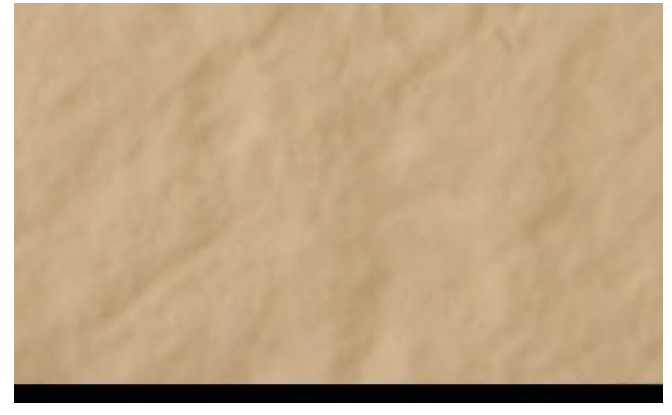

(b)

Figure 3.2: Pyrogel (a) AR 5401, (b) AR 5223

\subsubsection{Spaceloft}

Spaceloft blankets are commercially available flexible aerogel blankets made of silica gel and carbon black or Copolyolefin with an architecture based on poly (ethyleneterephthalate) [60]. Spaceloft materials are used in oil and natural gas industries, building and construction, outdoor gear and apparel [76].

During the transportation of Liquefied Natural Gas (LNG) crudes, wet gas or paraffinic crudes, the oil well and transfer lines are insulated to keep the temperature above that of paraffin crystal or hydrades in order to avoid a flow assurance problem. Spaceloft materials are used to insulate the oil and gas flowlines as well as the subsea pipelines. As a result of Spaceloft insulation, the offshore oil and gas industry conserves energy, saves a lot of money in oil and gas recovery, LNG storage and shipment, by using small pipes at reduced cost [77].

Spaceloft blankets are also used in pipe insulation, making it possible to use an outer pipe at least one standard pipe size smaller, while maintaining the same value of flow rate and thermal conductivity [16]. 
The total energy used in the USA for domestic heating in winter is only

$41 \%$. Of this, about $25-30 \%$ is wasted through unsealed spaces around windows $[78,79]$.

To increase the energy efficiency of windows, flexible aerogel blankets with better thermal performance are used to make the windows an important component of a building's energy and environmental systems [80]. Spaceloft blankets as thermal superinsulators are not only used in a window or door gasketing systems, but also in roofing, exterior walls, sheathing, curtain walls and thermal bridging [81]. For low pressure steam pipes, vessels and equipments, Spaceloft products are very useful in the thermal insulation process. The insulation of a school's hot water pipes in a confined space is shown in figure 3.3.This insulation provides a reduction in heat loss and protects personnel.

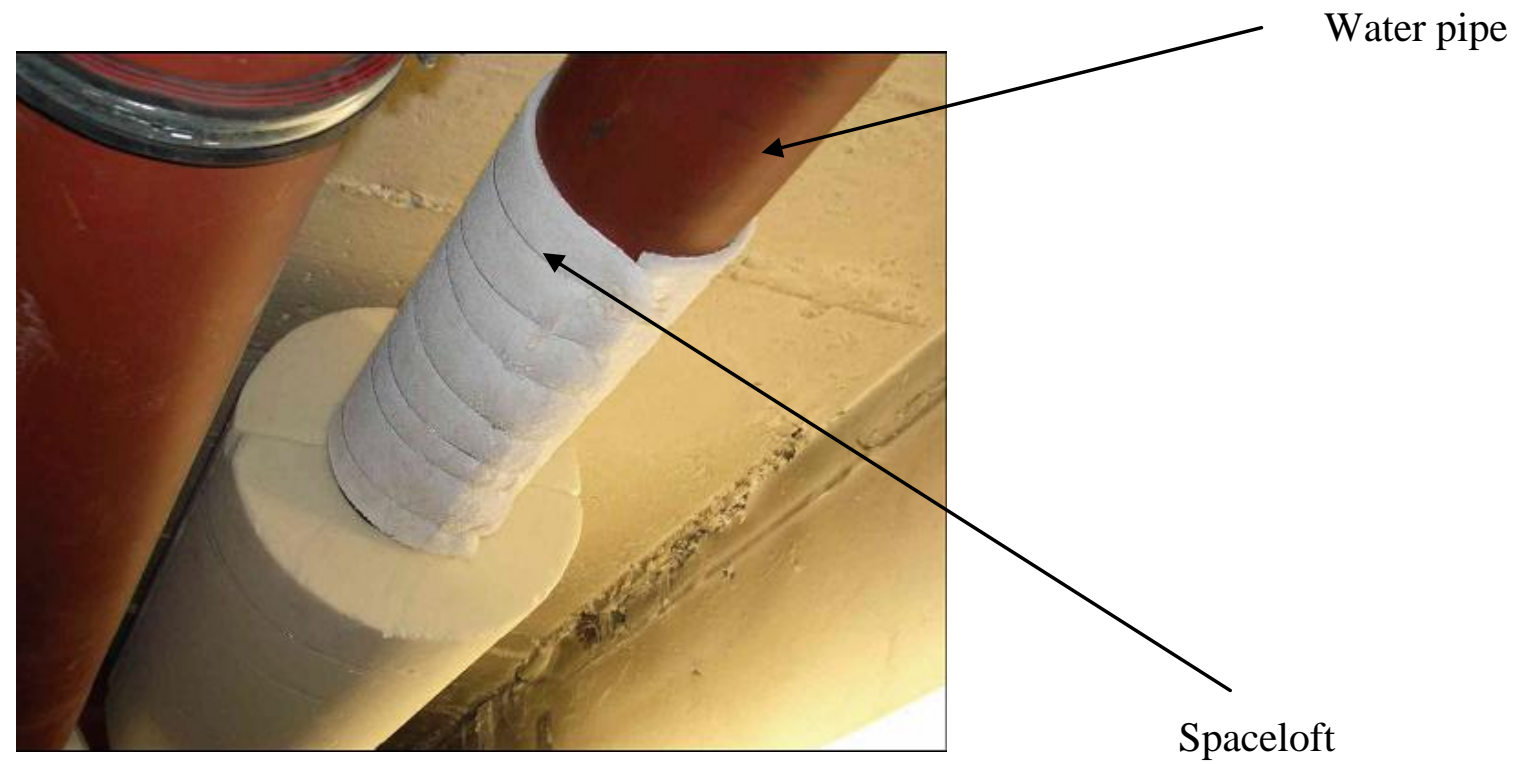

Figure 3.3: Spaceloft material insulation for hot water pipes [82]

Spaceeloft materials are used as thermal insulator in jackets, footwear, gloves, sleeping bags and pads. These flexible blankets are very useful during rain and snow because they withstand the penetration of water [83]. 


\subsubsection{Pyrogel}

The matrix composition of Pyrogel is made by mixing silica gel with oxidized Polyacrylonitrile or silicon dioxide and Zircon [60]. Pyrogel materials can be used as thermal insulation for a wide range of applications such as transportation, oil and gas, aerospace and military. Aerogels have been primarily developed for NASA. For example, during the Mars Pathfinder and the Exploration Rover missions, NASA used aerogels on space suits to minimize the heat lost and to provide adequate insulation [84, 22]. Aerogel based-blankets have also been employed to provide superior thermal insulation for a variety of applications in aircraft and helicopter applications. The use of Pyrogel materials leads to the reduction of thermal signature for hot engines on aircrafts and helicopters. Figure 3.4 shows a helicopter with and without Pyrogel blankets. In this experiment, The IR signature measurements of both helicopters were performed using a Bomem M 254 radiometer. The results obtained exhibit a $40 \%$ reduction of IR signature when the aft portion of the helicopter's engine is attached with an encapsulated Pyrogel blanket [28].
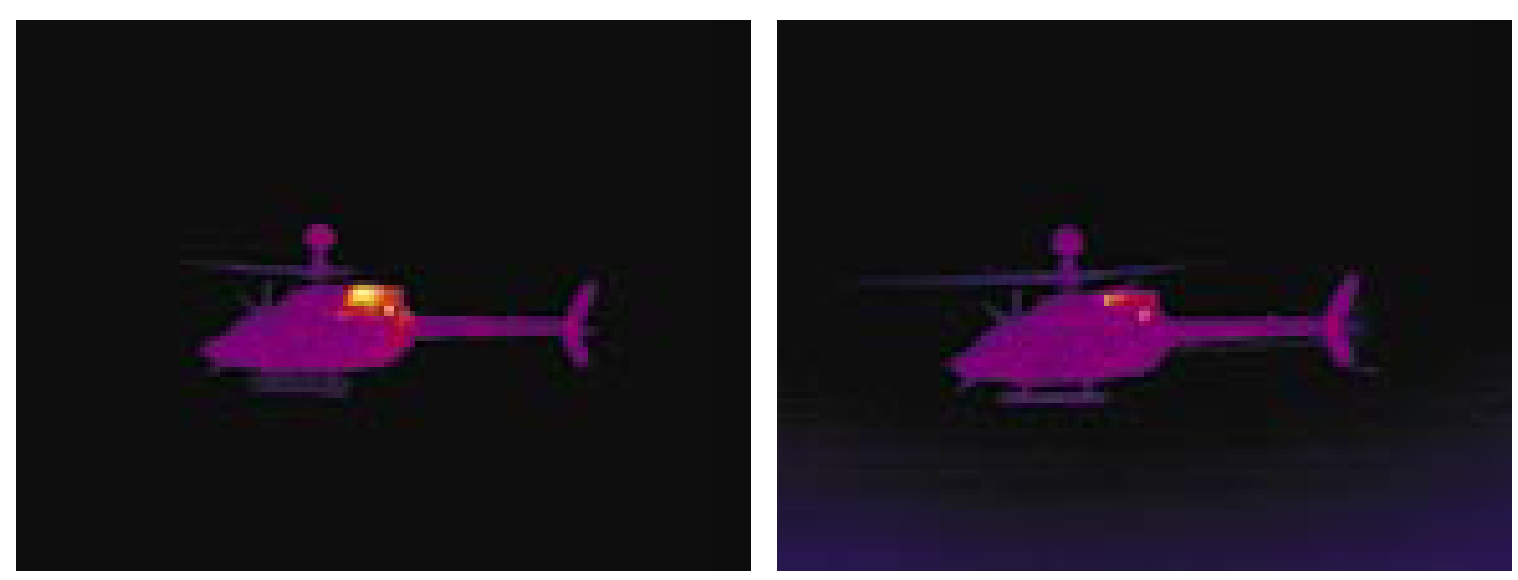

Figure 3.4 : Thermal images of a helicopter: without (left) and with Pyrogel blankets (right) [28] 
In the military market, Pyrogel blankets provide thermal and fire protection for armored vehicles and military shelters. In severe conditions, insulation military shelters saves up to $70 \%$ of the fuel quantity needed to heat or cool them [28].

Another interesting application of Pyrogel materials is for medium and high pressure steam pipes, vessels and equipment applications. Figure 3.5 exhibits a high pressure vessel insulated with Pyrogel materials.

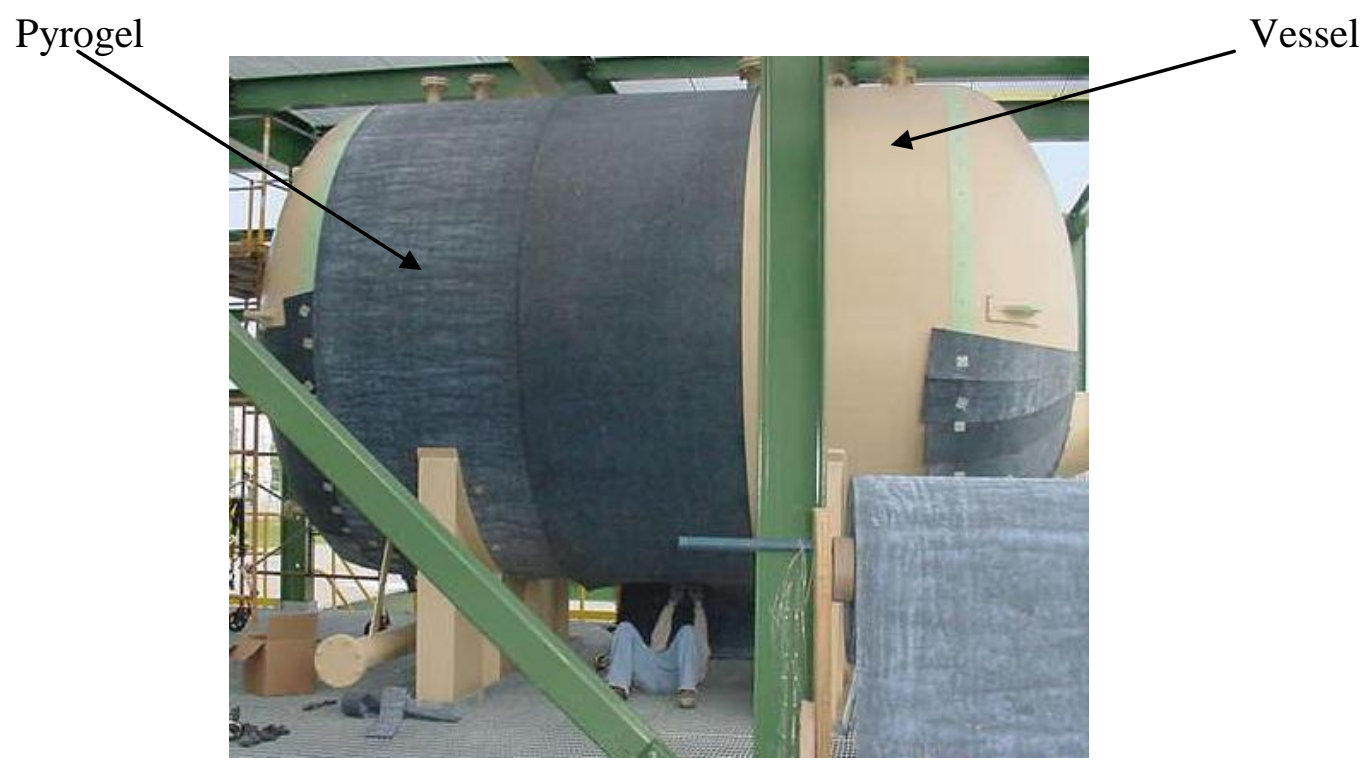

Figure 3.5: Large insulated vessel with Pyrogel thermal insulation [85]

\subsection{Scanning Electron Microscopy (SEM)}

The flexible aerogel blankets samples were viewed under a scanning electron microscope (SEM) which provides the fine details of the interior structures of these blankets. SEM produces highly magnified and stereo three dimensional (3D) images of the surfaces of these blankets. SEM helps to observe features such as microstructure, fracture surfaces and interfaces of the aerogel blanket materials. SEM is by far the most popular technique for fractographic studies of materials. Unlike an optical microscope, 
the SEM uses an electron beam rather than light and outlines the contrast of the created image which is based on a flexible aerogel blanket's compositions variations [86-88].

Figure 3.6 shows the SEM used during this study.
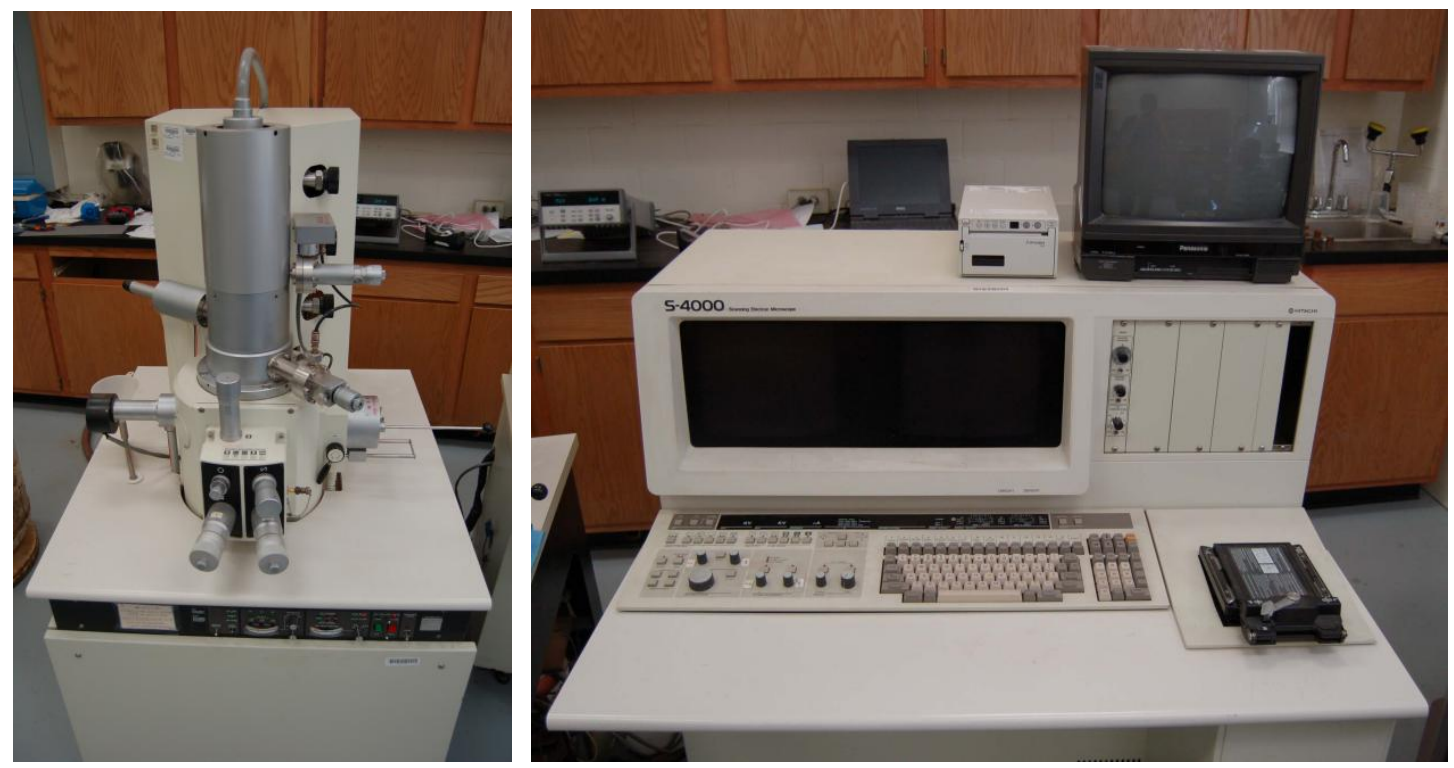

Figure 3.6: Photograph of scanning electron microscope Hitachi S-4000

This SEM instrument is equipped with two main features, the electron column and the electronics console. While the electronics console contains the control panel electronic, the electronic power supplies and the scanning module, the electron source encompasses the field emission gun, the sample vacuum chamber, the magnetic focusing lenses and the stage region located at the bottom of the column.

The flexible aerogel blanket samples were prepared using the conductive coating method. This method was chosen to increase the contrast of the surface images of the blankets and to neutralize the deposit of static electric fields on the sample [89]. The flexible blankets specimens were coated with a conductive thin film of gold (Au) under vacuum in a sputter coater for 2 minutes. The thickness of the thin gold film was $20 \mathrm{~nm}$. 
Before the coating process, the blanket sample was fixed to a stub using carbon paint. After being coated, the stub wass placed in an appropriate holder and tightened firmly with screws. The sample holder was adjusted to match the gauge rod's height. The next step was to remove the protective cover from the vented airlock. Before evacuating the airlock, the specimen exchange rod was placed over the airlock portal. After pulling out the chamber airlock door, the specimen holder was introduced into the specimen exchange chamber. The button "EVAC" was depressed and the specimen exchange chamber put under high vacuum. The specimen exchange rod was unscrewed from the sample holder and pulled back into the airlock. After closing the chamber airlock, air was pushed to vent the airlock and make the specimen vacuum stable [90]. Image acquisition was accomplished through window software.

Mechanical properties of the flexible aerogel blankets were investigated using the SEM in a timely manner. The flexible aerogel blanket materials were viewed under SEM before and after completion of tensile and bending tests. Before the mechanical testing, the blankets were cut into very small sections and observed under SEM. A small section from the middle part of the blanket strip piece that was fractured after the tensile test was also scanned using the SEM. After being subjected to 10000 cycles in the three point bending test, the surface of a small area in the middle part of aerogel blanket pieces was studied using SEM [91].

\subsection{Bending test}

Several bending tests were performed on the aerogel blanket materials to analyze the characteristics of their bending behavior. Three point bending tests were used to study the mechanical properties of flexible aerogel blankets [92]. Before starting the 
experiment, the testing machine was calibrated so that the initial load and the initial displacement was zero. The width and the thickness of a strip of an aerogel blanket were measured at several locations.

The dimensions of the strip were $60 \mathrm{~mm}$ X $20 \mathrm{~mm}$. The rectangular crosssectional specimen was placed on two parallel supporting pins. Indeed, the sample was aligning midway between the supports. In the middle part of the specimen, a loading force was applied by a loading pin. The supporting and loading pins needed to be mounted in such way that:

- Their free rotation around the axis parallel to the pin axis and around the axis parallel to the specimen axis was assured

- The loading of the specimen was uniform

- There was no friction between the specimen and the supporting spins

After selecting the testing mode (flexural), the samples parameters were successfully processed to the software and the "start test" button pushed. After the specimen had made one (1) cycle, the stop button was pressed. The bending tests were conducted at room temperature $25^{\circ} \mathrm{C}$. The crosshead speed was $1 \frac{\mathrm{mm}}{\mathrm{min}}$ and the fulcrum span was $40 \mathrm{~mm}$. The three point bending experiments were conducted on an INSTRON Model 4411 (Instron Corporation, Norwood, MA) universal testing machine as shown in figure 3.7 . 


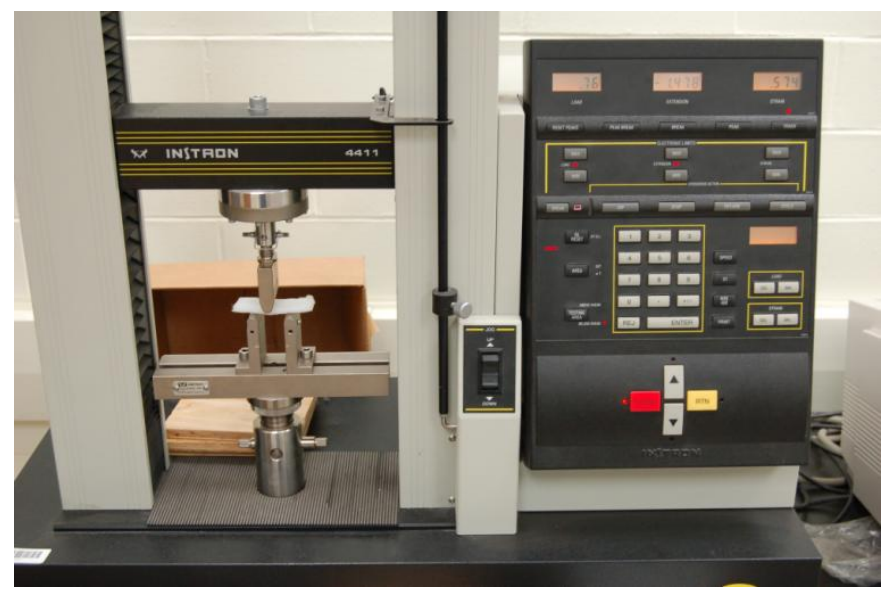

Figure 3.7: Universal testing machine INSTRON 4411

During the three point bending test, the loads acted perpendicular to the longitudinal axis of the insulating blanket. Therefore, the flexible aerogel blanket sample could be modeled as a beam, as shown in figure 3.8(a). Figure 3.8 (b) shows the deformation of the beam axis into a part of a circle of radius $\rho$.

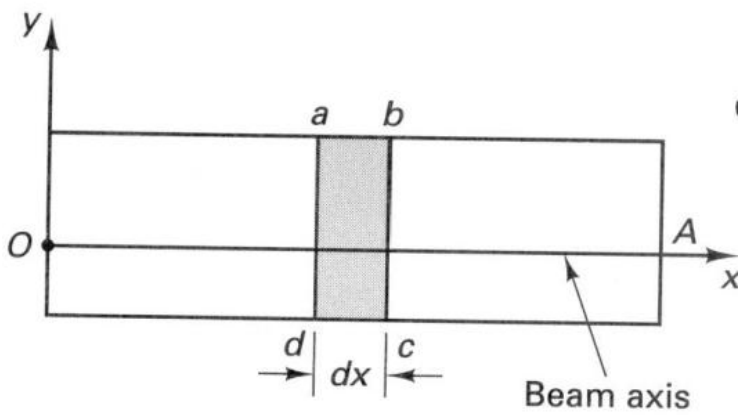

(a)

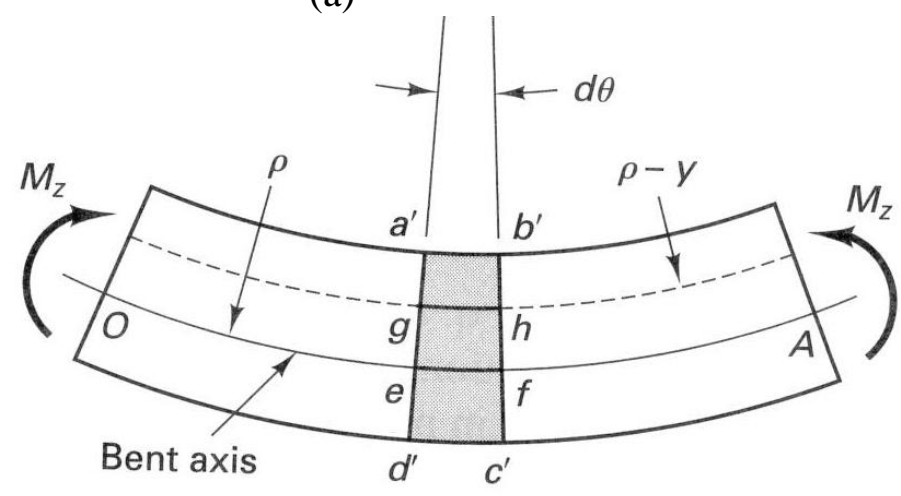

(b)

Figure 3.8: Behavior of an elastic beam in bending 
For an element having a fiber length of e-f and an infinitesimal angle $d \theta$, the beam axis can be expressed as follows:

$$
d s=\rho . d \theta \quad \text { Hence, } \quad \frac{d \theta}{d s}=\frac{1}{\rho}=k
$$

A more general expression of the curvature is:

$$
k=\frac{d^{2} y / d x^{2}}{+(d y+d x)^{2}-}
$$

The slope $\mathrm{dy} / \mathrm{dx}$ is very small and its square even smaller and can be neglected. With these simplifications, equation 3.2 becomes:

$$
k=\frac{d^{2} y}{d x^{2}}
$$

Identically, the fiber length $\mathrm{g}$-h located on a radius $\rho-y$ is given as:

$$
\hat{d \hat{u}}=(\rho-y) d \theta-\rho_{d} \theta=-y d \theta
$$

By dividing equation 3.4 by ds and approximating $d \hat{u} / d s$ by $d u / d x$, the normal strain is obtained:

$$
\varepsilon_{x}=-y \cdot k
$$

By using Hooke's law, the normal longitudinal stress is obtained as:

$$
\sigma_{x}=E \cdot \varepsilon_{x}=-E \cdot y \cdot k
$$

To solve the beam flexure problem, two nontrivial equations of equilibrium must be brought in. The sum of all forces at a section in the $\mathrm{x}$-direction and the sum of the resisting moments must vanish, one has 
$\sum F_{x}=\int \sigma_{x} d A=0 \quad \sum M_{0}=M_{Z}-\int E \cdot k \cdot y \cdot d A \cdot y=0$

Equation 3.7

A

Recognizing that both $\mathrm{E}$ and $\mathrm{K}$ are constant in pure bending of beams, the external

moment would read:

$$
M_{Z}=k \cdot E \cdot \int_{A} y^{2} d A=k \cdot E \cdot I_{z}=\frac{d^{2} y}{d x^{2}} \cdot E \cdot I_{z}
$$

Equation 3.8

This equation can be used to derive an expression of $\mathrm{k}$ (kappa) which is then substituted into equation 3.6, this yields

$$
\sigma_{x}=-\frac{M_{Z}}{I_{Z}} y \quad \text { or } \quad \sigma_{x}=+\frac{M_{y}}{I_{y}} Z
$$

The expression for the maximum normal stress with the aid of equation 3.7 can be rewritten as:

$$
\sigma_{\max }=\frac{M}{I} \cdot\left|y_{\max }\right|=\frac{M c}{I}=\frac{M}{I / c}=\frac{M}{S}
$$

The centroid of the gray section in figure 3.7 (a) lies at the intersection of the two axes of symmetry. Here it is convenient to take dA as the length of the beam dy. Hence,

$$
I_{x}=I_{0}=\int_{A} y^{2} d A=\int_{-h / 2}^{+h / 2} y^{2} b d y=\frac{b h^{3}}{12}
$$

The section modulus for a rectangular section in algebraic form is: 


$$
S=\frac{I}{c}=\frac{b h^{3}}{12} \frac{2}{h}=\frac{b h^{2}}{6}
$$

Equation 3.12

The flexure formula becomes:

$$
\sigma_{\text {max }}=\frac{6 \cdot M}{b h^{2}}
$$

Equation 3.13

The maximum value of the bending moment is given as:

$$
M=\frac{F \cdot L}{4}
$$

The center deflection can be determined from the differential equation for the elastic curve given in equation 3.8:

$$
D=\frac{F \cdot L^{3}}{48 \cdot E \cdot I}
$$

The modulus of elasticity can be determined by rearranging equation 3.13 and equation 3.9:

$$
E_{B}=\frac{F \cdot L^{3}}{4 \cdot b \cdot h^{3} \cdot D}=\frac{a \cdot L^{3}}{4 \cdot b \cdot h^{3}}
$$

where a, the slope of the tangent to the initial straight line portion of the load deflection curve is defined as:

$$
a=\frac{F}{D}
$$

The bending tests were also performed by repeating the loading and unloading processes on the aerogel blanket strips [93]. The cyclic loading was 10 cycles, 100 cycles, 1000 cycles, 2000 cycles, 5000 cycles, 8000 cycles and 10000 cycles with 
a crosshead speed of $15 \frac{\mathrm{mm}}{\mathrm{min}}$. Before and after each cycle, the sample was removed from the INSTRON machine and placed in a laboratory balance to measure the mass.

\subsection{Tensile test}

To measure the basic mechanical properties of the flexible aerogel blankets materials, the tensile test was widely used [94]. During the tensile test, the aerogel blankets were cut into strips of $35 \mathrm{~mm}$ by $6 \mathrm{~mm}$ and pulled until they fractured. The samples were placed between the lower and upper grips of the tensile tester, which were then firmly tightened.

Before starting the tensile experiment, the length of the samples between the edges of the grips was measured to determine the grip separation or the gauge length. After recording the geometrical parameters of the sample in the series IX software, the tensile experiments of the blankets were performed. Like the bending test, the tensile tests were conducted at room temperature and on an INSTRON Model 4411.

The crosshead speed was $1 \frac{\mathrm{mm}}{\min }$. In this test, the sample elongation and applied load were acquired using the series IX data acquisition software. Stress and strain were calculated from these values using the following formulas:

$\sigma=\frac{F_{i}}{w \times \delta} \quad$ Engineering stress

Equation 3.19

$\varepsilon=\frac{l_{i}-l_{0}}{l_{0}} \quad$ Engineering strain

Equation 3.20

The stress-strain values obtained were used to construct a stress-strain curve. 


\subsection{Measurement of thermal resistance}

To evaluate the thermal resistance of the flexible aerogel blanket materials, the bottom surface of each specimen was exposed to a hot plate and the top surface to air [95]. The hot plate used is shown in figure 3.9.

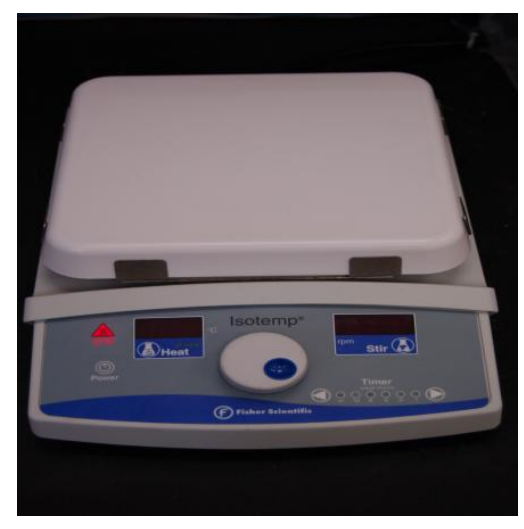

Figure 3.9: Fisher Scientific Isotemp Digital hotplate

The temperature of the top and the bottom surfaces of the tested blanket were measured in the middle section of the specimen using two $\mathrm{J}$ type thermocouples, one for each surface. The data from the thermocouples were obtained using a data logger interface with VEE software (agilent technologies). The"Heat" button was pressed and the main button turned clockwise until the selected temperature was obtained. Then, the "heat" button was pressed again. When the hot plate reached the selected temperature, it became stable and steady.

The first thermal resistance measurement of the flexible aerogel blankets was performed at an unsteady state. The initial and final temperatures of the hot plate were set at $25^{\circ} \mathrm{C}$ and $125^{\circ} \mathrm{C}$ respectively. While the temperature of the hot plate was changing, the temperature of the top and the bottom surface of the flexible aerogel blankets were 
measured at intervals of time.

The second category was thermal resistance measurement of the insulation blanket at a steady state. The hot plate was set up for the following temperatures: $60{ }^{\circ} \mathrm{C}, 80{ }^{\circ} \mathrm{C}, 100^{\circ} \mathrm{C}, 125{ }^{\circ} \mathrm{C}$ and $150{ }^{\circ} \mathrm{C}$. For each increase, the temperature of the top and the bottom surfaces of the aerogel blankets were determined at a steady state. The temperature difference across the sample and the normalized relative temperature drop [95] were calculated using the following formulas respectively:

$$
\begin{aligned}
& \Delta T=T_{h . s .}-T_{c . s .} \\
& \Delta T_{\text {normalized }}(\text { Drop })=\frac{T_{c . s .}-T_{h . s .}}{T_{h . s .}}
\end{aligned}
$$

The thermal resistance of the flexible aerogel blankets was also related to their mechanical properties. Thermal resistance tests were performed and linked with tensile and bending tests. A sample with the same dimension as the ones tested during the bending test was placed on the hot plate and its top and bottom surface temperatures measured. Then, the sample was subjected to 1 cycle of three point bending test. Finally, the sample was removed from the testing machine and its surface temperatures measured again. This procedure was repeated for 10, 100, 1000, 2000, 5000, 8000 and 10000 cycles. For the tensile test, the thermal resistance of the flexible aerogel blanket was measured before and after the following displacements: $0 \mathrm{~mm}, 0.25 \mathrm{~mm}, 0.75 \mathrm{~mm}$, $1.25 \mathrm{~mm}, 1.75 \mathrm{~mm}, 2.25 \mathrm{~mm}$ and $2.8 \mathrm{~mm}$. In these experiments, the hot plate temperature was set to a maximum of $150^{\circ} \mathrm{C}$. 


\subsection{Thermogravimetric analysis investigations}

The weight loss measurements and the kinetics of the thermal degradation were performed using a Perkin Elmer thermogravimetric analyzer, Pyris 1 TGA. This TGA analyzer has four sections which are the main frame, the console, the pressure/flow controller and the computer system. The support stand, the elevator, the furnace and pressure balance constitutes the main frame. The console consists of a visual display of temperature and weight which are then recorded and stored in the computer system. The pressure/flow controller maintains the flow rates of the gases. The TGA test starts by placing the aerogel blanket sample in the aluminium pan and then hanging down the pan and make the furnace to move up and heat the pan.

Two independent experiments were set. The first experiment was devoted to measure the weight loss of flexible aerogel blankets in relation to temperature changing from room temperature to $850{ }^{\circ} \mathrm{C}$ at a heating rate of $10{ }^{\circ} \mathrm{C} / \mathrm{min}$. In the second experiment, the flexible aerogel blanket specimens were kept at a constant temperature $T=375^{\circ} \mathrm{C}$ during 450 minutes. For the two sets of experiments, the TGA was performed under (pressurized) air and nitrogen atmosphere separately.

\subsection{Contact angle measurements}

Deionized water drops were placed on the surface of each aerogel blanket and a photo was taken from the side using a travelling microscope. The angle between the water droplet and the surface of the blanket was measured from the microscopy images of the water drops. This angle is also called the contact angle, $\theta_{0}$, and can be obtained using the following formula [96]: 


$$
\theta_{0}=2 \tan ^{-1}\left[\frac{2 h_{0}}{d_{0}}\right]
$$

Equation 3.23

The height of the droplet, $h_{0}$, and the base length of the droplet, $d_{0}$, are obtained using the microscopy images.

Alternatively, the contact angle can be obtained using image $\mathrm{j}$ software by downloading a package called "drop_analysis.zip". This package was developed by Stadler [97] and is used for measuring with high accuracy contact angles based on the Bspline snakes (active contours) and the low bond asymmetric drop shape analysis (ADSA approach) methods. In the B-spline snake approach, the whole drope shape is used to unify the aspects of locality of the contact angle and provide global information about the contact angle measurement. Derived from a first order perturbation solution of the Laplace equation for axisymmetric drops, the low bond asymmetric drop shape analysis method used the whole drop profile to measure the contact angles.

\subsection{Numerical modeling using input from experimental observations}

The numerical experiments were performed by finite element analysis using the ANSYS software package for computer simulation. The input data for the finite element method simulation were selected in connection with experimental results. Examination of flexible aerogel blankets with an SEM provided a wealth of information about their various components, and their geometry (shape), distribution and orientation inside the blanket. The mechanical and thermal properties of each constituent the flexible aerogel blanket were added as part of the finite element analysis component. The volume fraction of the constituent the flexible aerogel blanket, an important parameter of the finite 
element method, was obtained using the thermogravimetric and SEM image analysis results. Tensile testing, three point bending and fatigue damages evolution was monitored by the aid of the SEM. The results provided a deeper understanding of the causes and mechanisms of deformation and degradation of the blankets. The finite element model was developed using initial and boundary conditions provided during the thermal testing. 


\section{Chapter 4 Experimental Results and Discussions}

\subsection{SEM Results}

\subsubsection{Untested aerogel blankets}

More detailed information related to the microstructure of untested flexible aerogel

blankets were obtained from SEM images. SEM photographs of Pyrogel materials are shown in figure 4.1 .

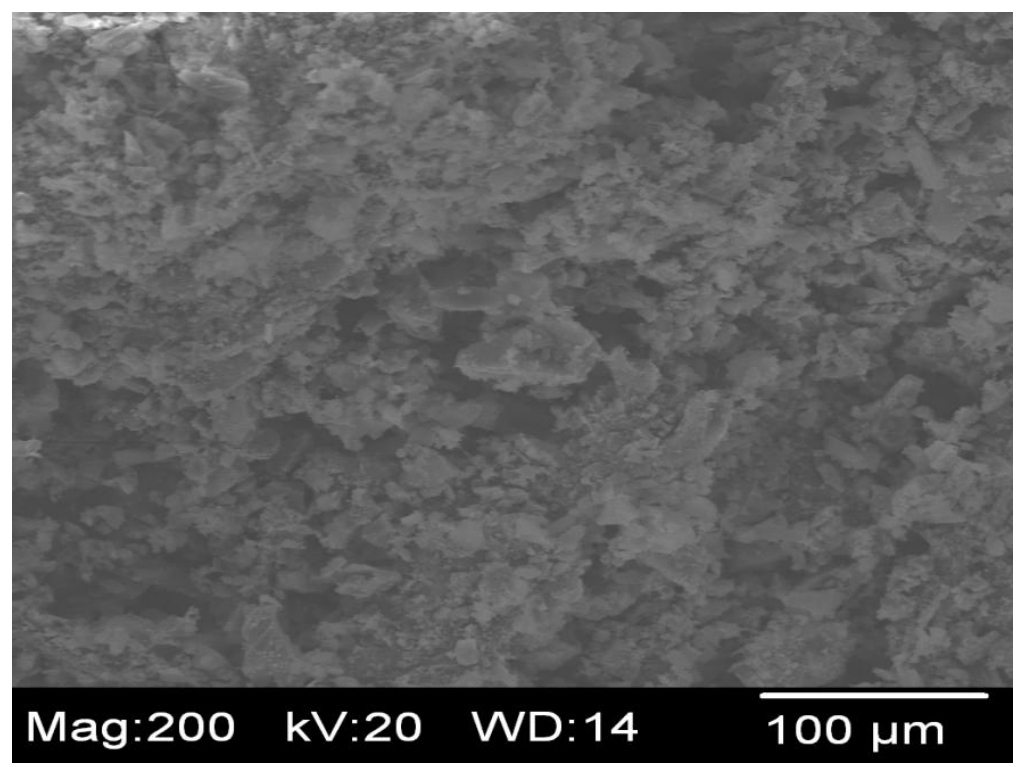

(a)

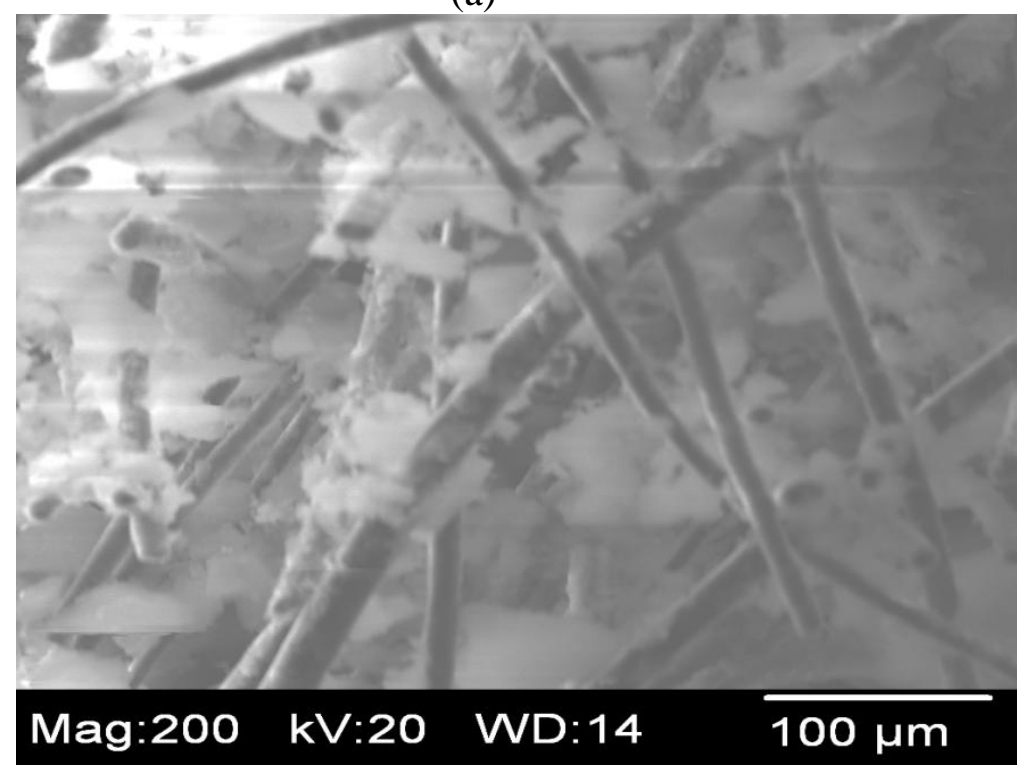

(b) 


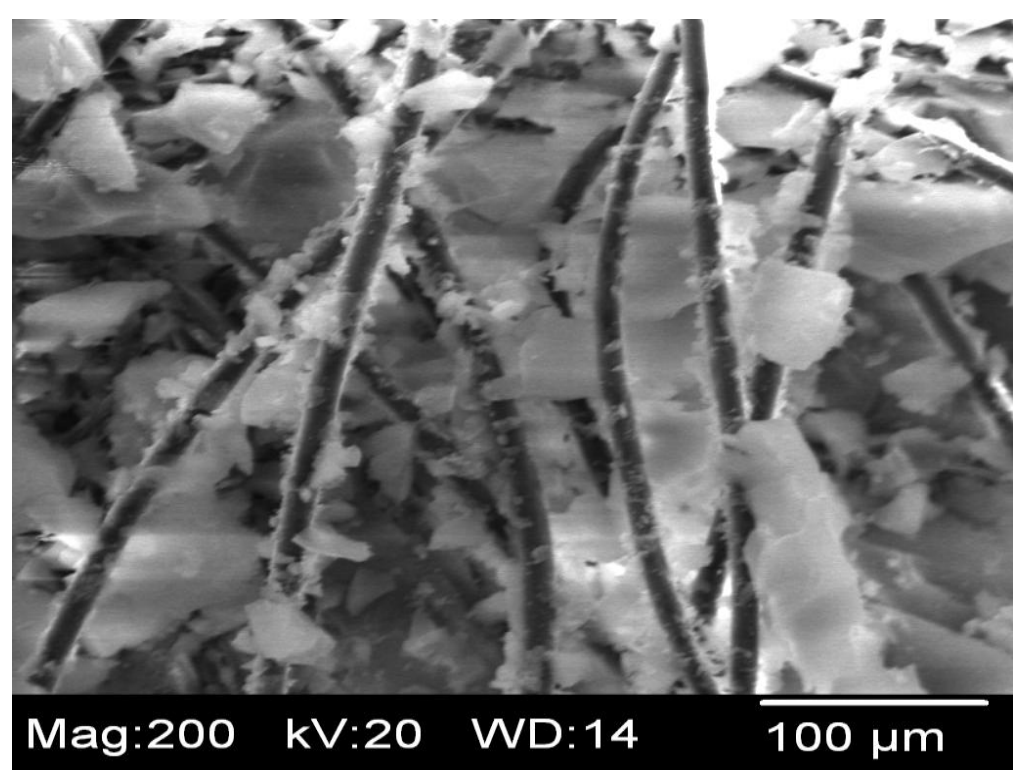

(c)

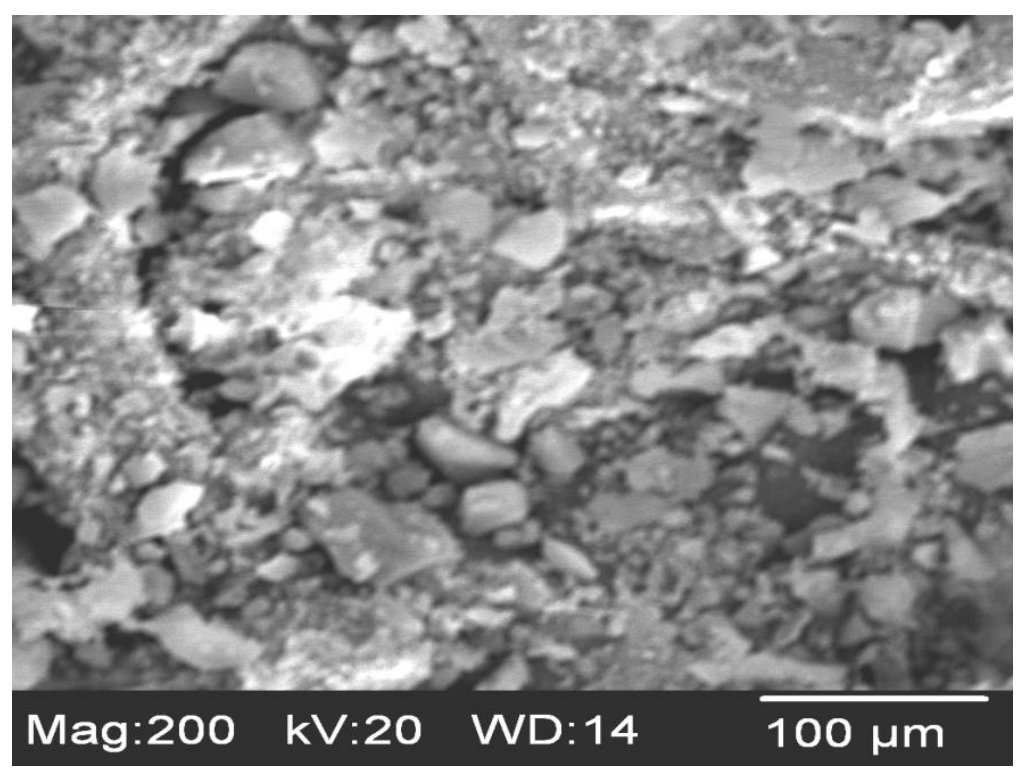

(d) 


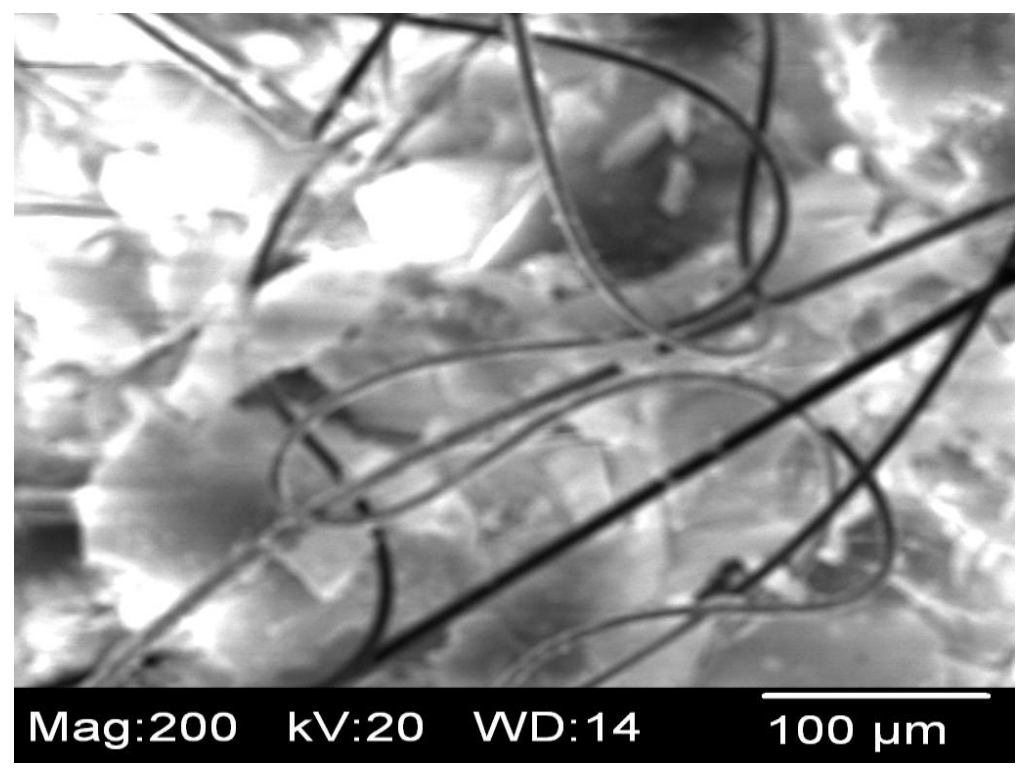

(e)

Figure 4.1: SEM images of untested Pyrogel blanket materials (a) AR 5403X; (b) AR 5400; (c) AR 5401; (d) AR 5401X; (e) AR 5223

Figure 4.2 shows the microscopy images of Spaceloft material surfaces.

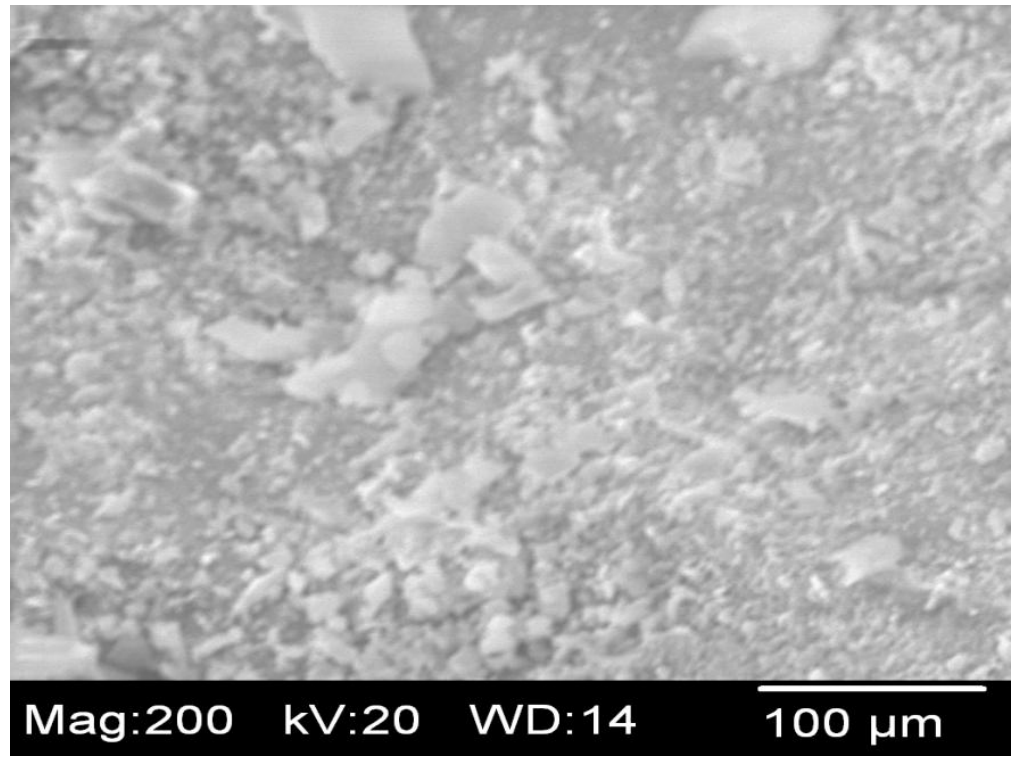

(a) 


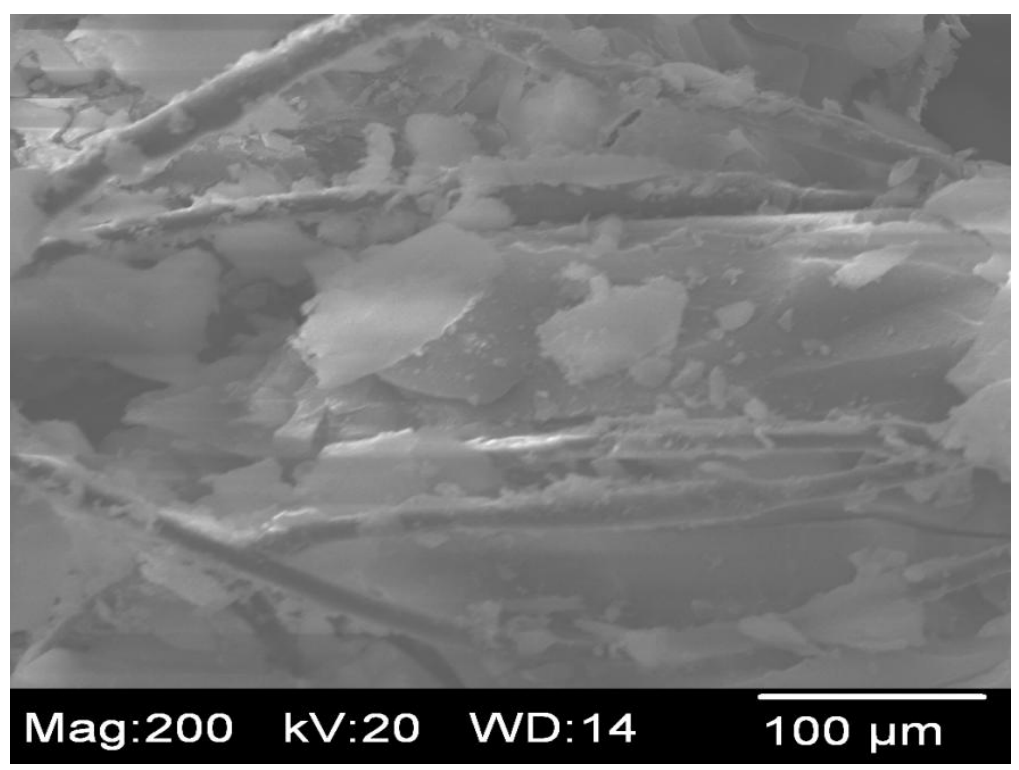

(b)

Figure 4.2: SEM images of untested Spaceloft blanket materials

(a) AR 3103X; (b) AR 3103;

In the SEM images of Pyrogel and Spaceloft blankets, fibers, the air pockets and aerogel particles can be identified. During the incorporation of fibers into the silica aerogels or during the manufacturing of aerogel blankets, air or other volatiles are trapped in the blanket material. The existence of the trapped air or other volatiles in the perfect blanket is in the form of pockets or microvoids which maybe visible without destroying the blanket.

\subsubsection{Bending}

The SEM photographs of pyrogel blankets were taken after being tested to a three point bending test can be seen in figure 4.3 . 


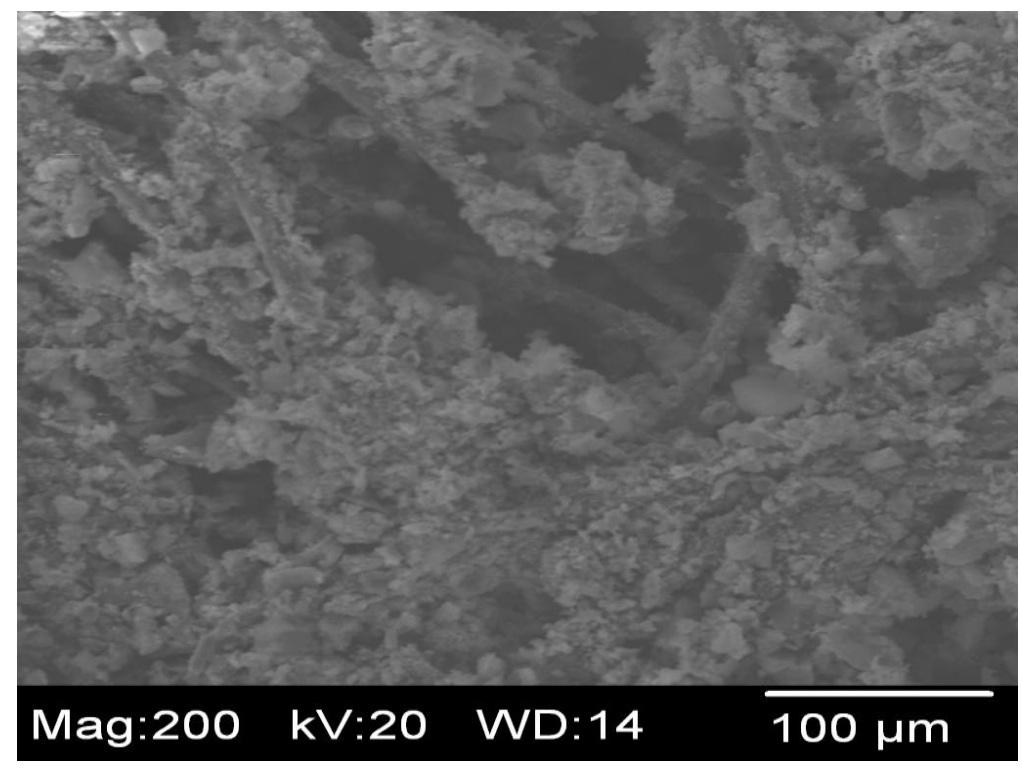

(a)

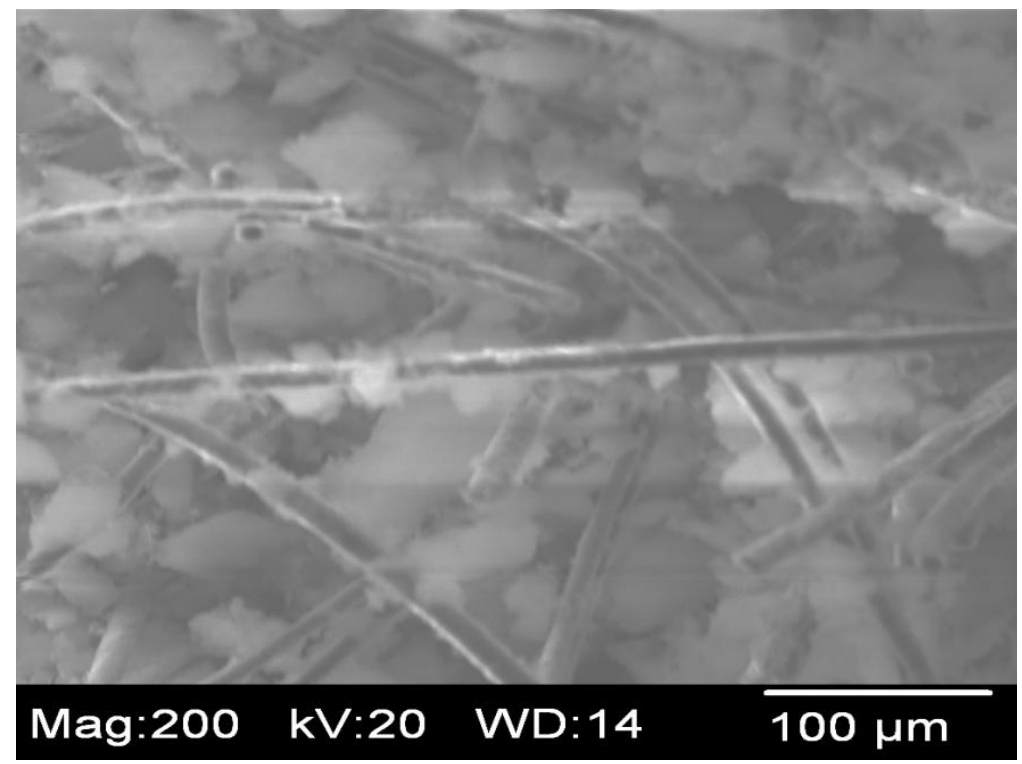

(b) 


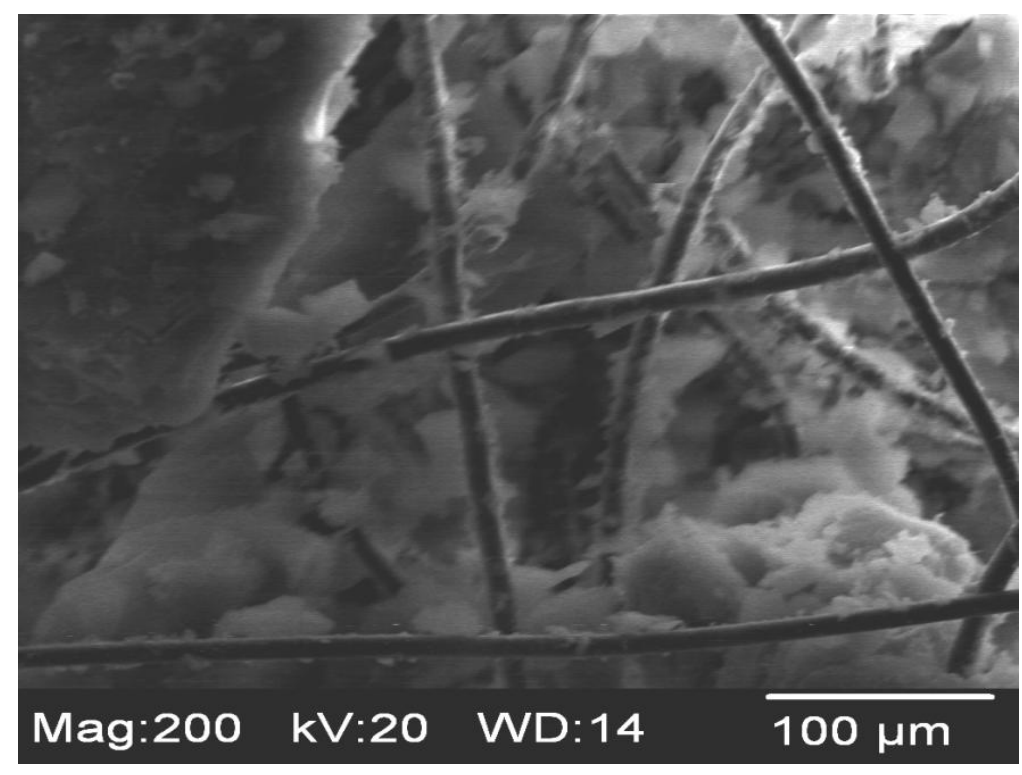

(c)

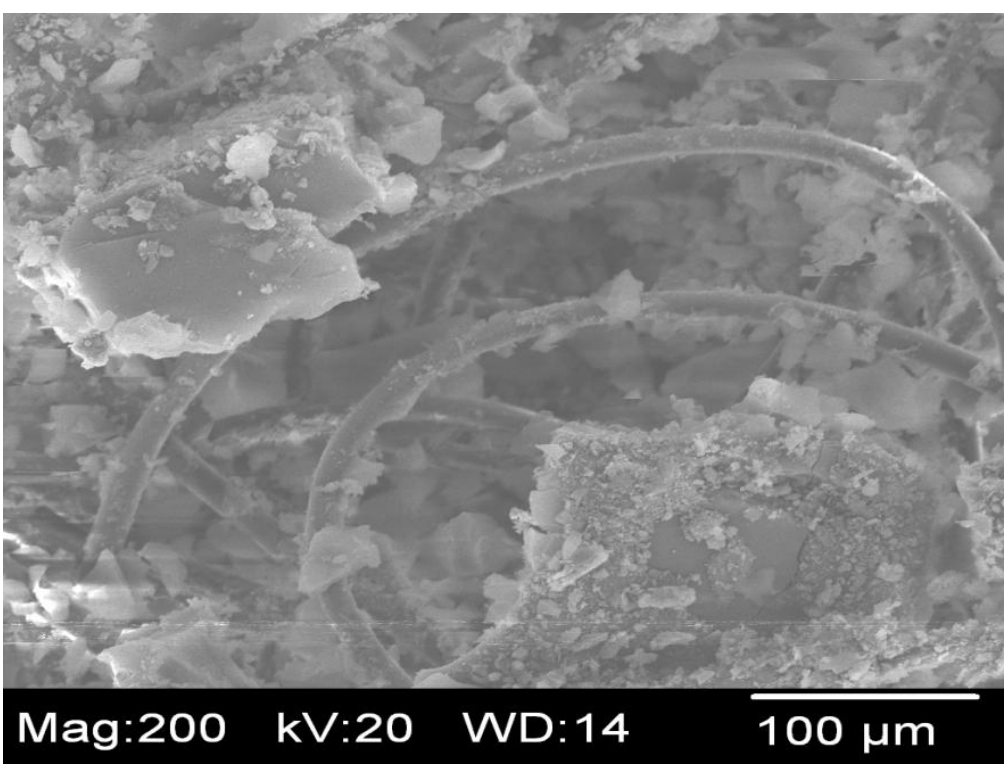

(d) 


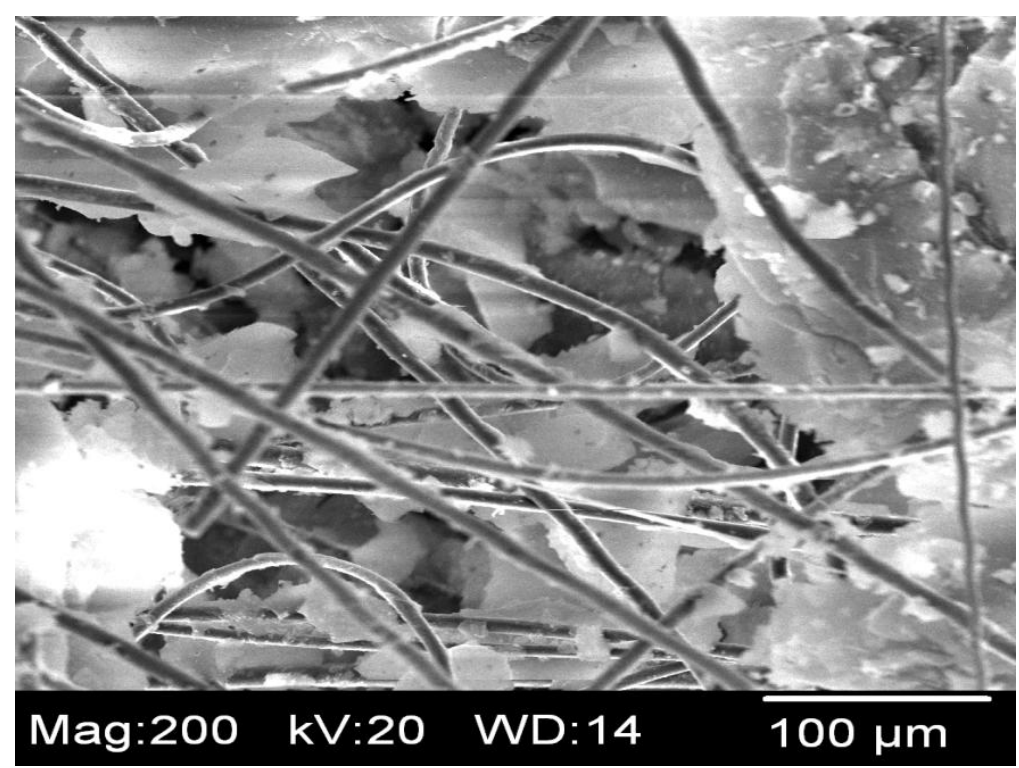

(e)

Figure 4.3: SEM images of three points bending Pyrogel material surfaces (a) AR 5403X; (b) AR 5400; (c) AR 5401; (d) AR 5401X; (e) AR 5223

Figure 4.4 showed the SEM micrographs of spaceloft blankets after being subjected to a cyclic uniaxial three point bending load (10,000 cycles).

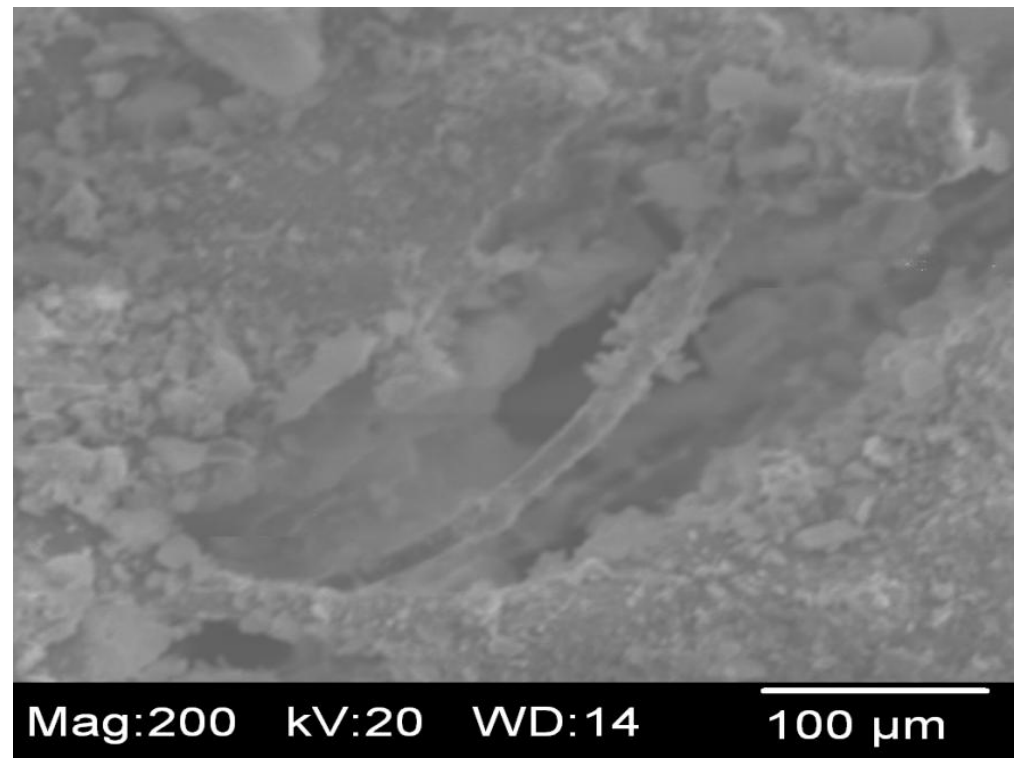

(a) 


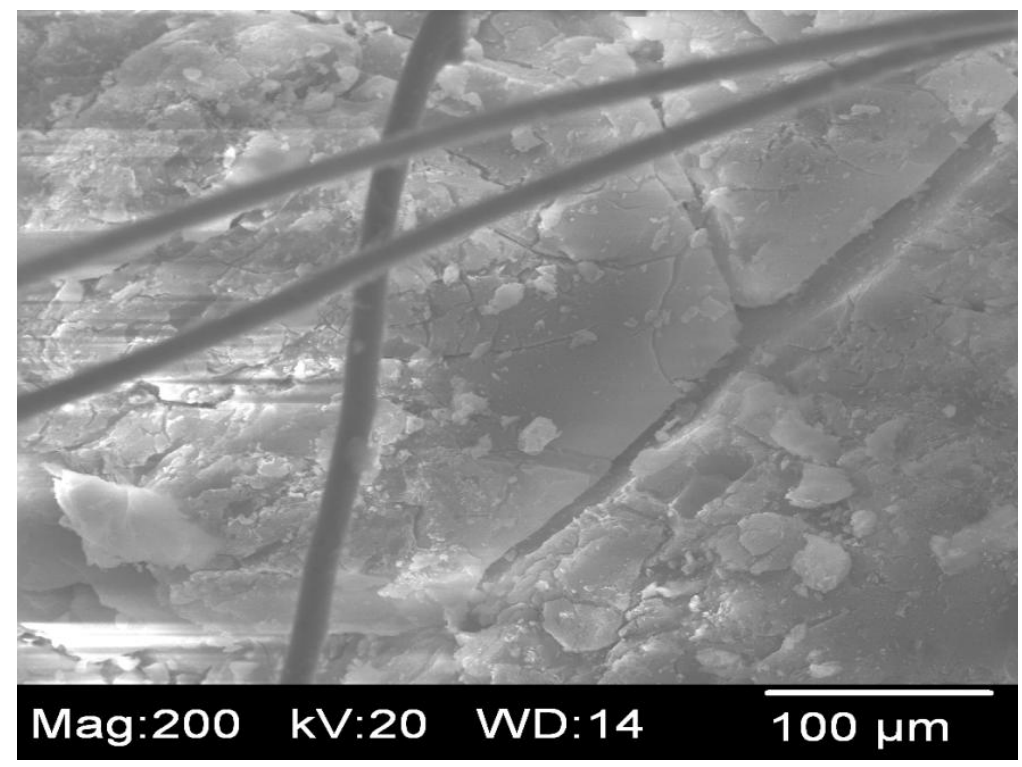

(b)

Figure 4.4: SEM images of three points bending Pyrogel material surfaces (a) AR 3103X; (b) AR 3103

\subsubsection{Tensile}

Figure 4.5 and 4.6 show SEM pictures of a flexible aerogel blanket after undergoing a tensile test.

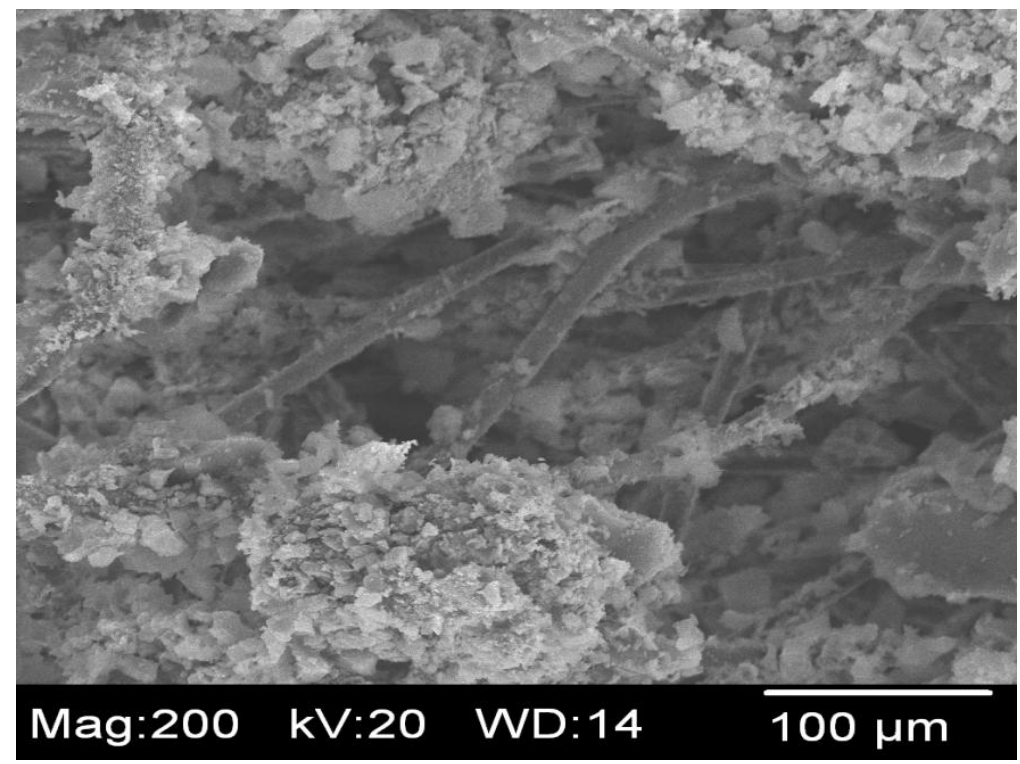

(a) 


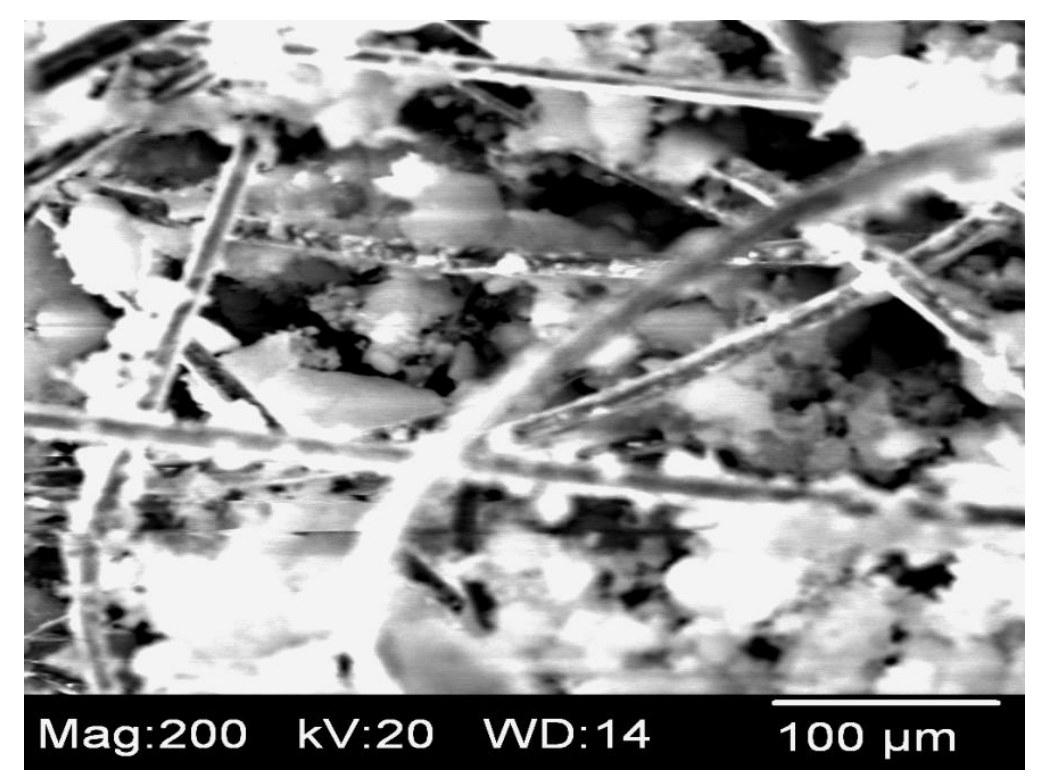

(b)

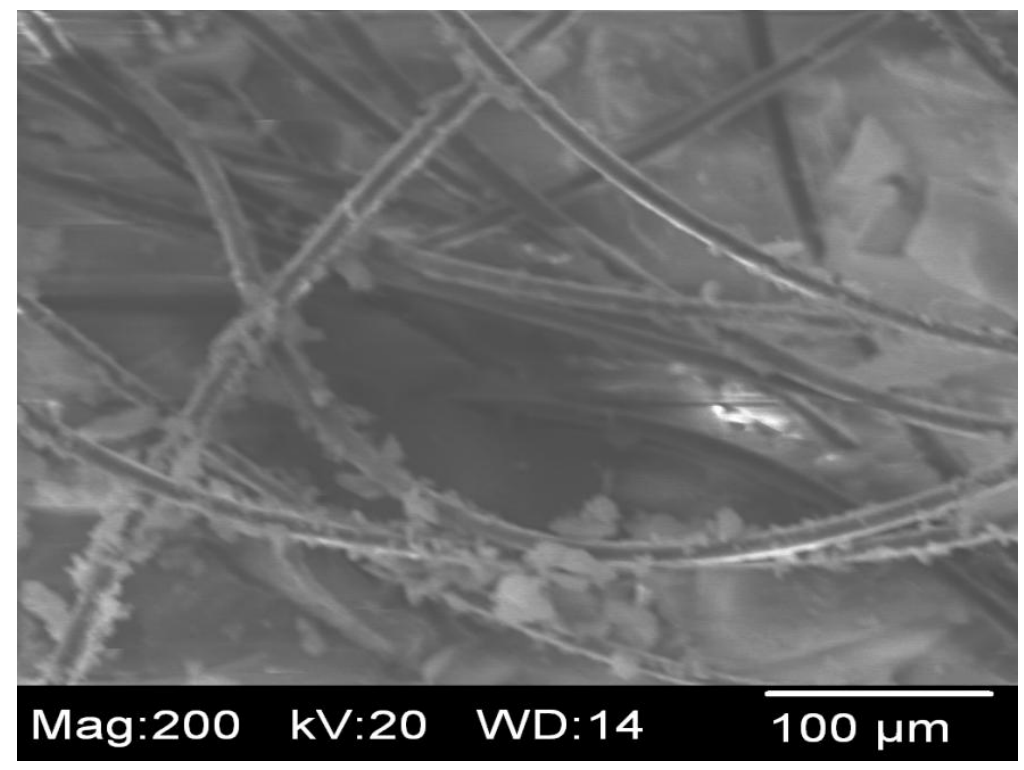

(c) 


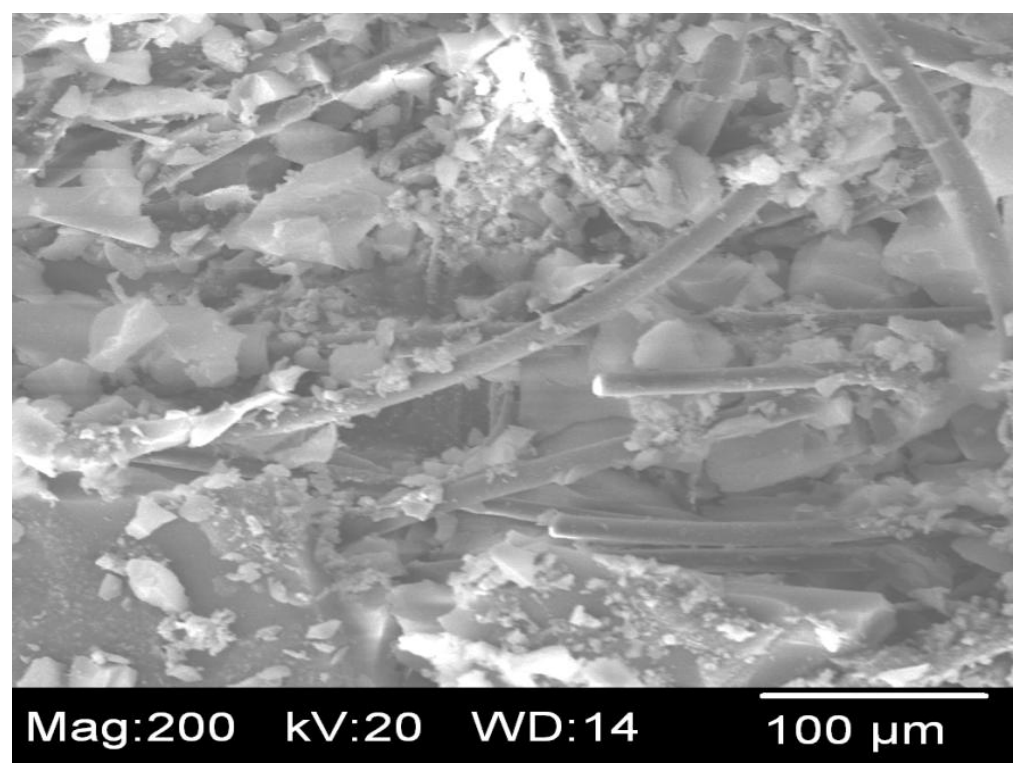

(d)

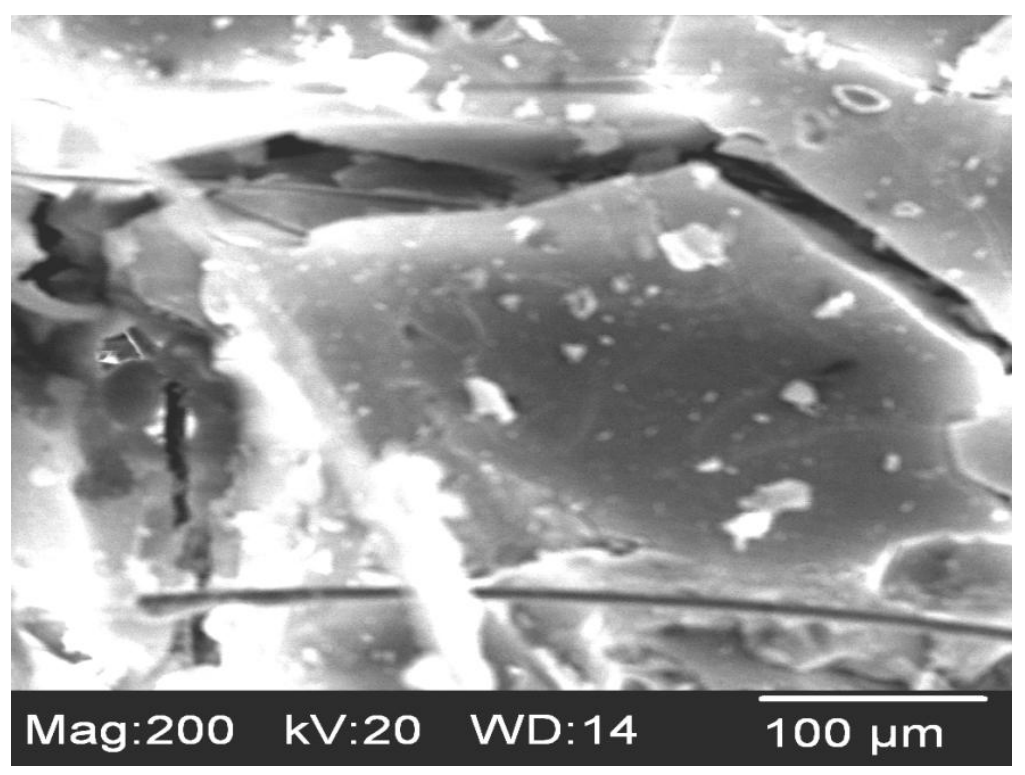

(e)

Figure 4.5: SEM images of tensile Pyrogel material surfaces (a) AR 5403X; (b) AR 5400; (c) AR 5401; (d) AR 5401X; (e) AR 5223 


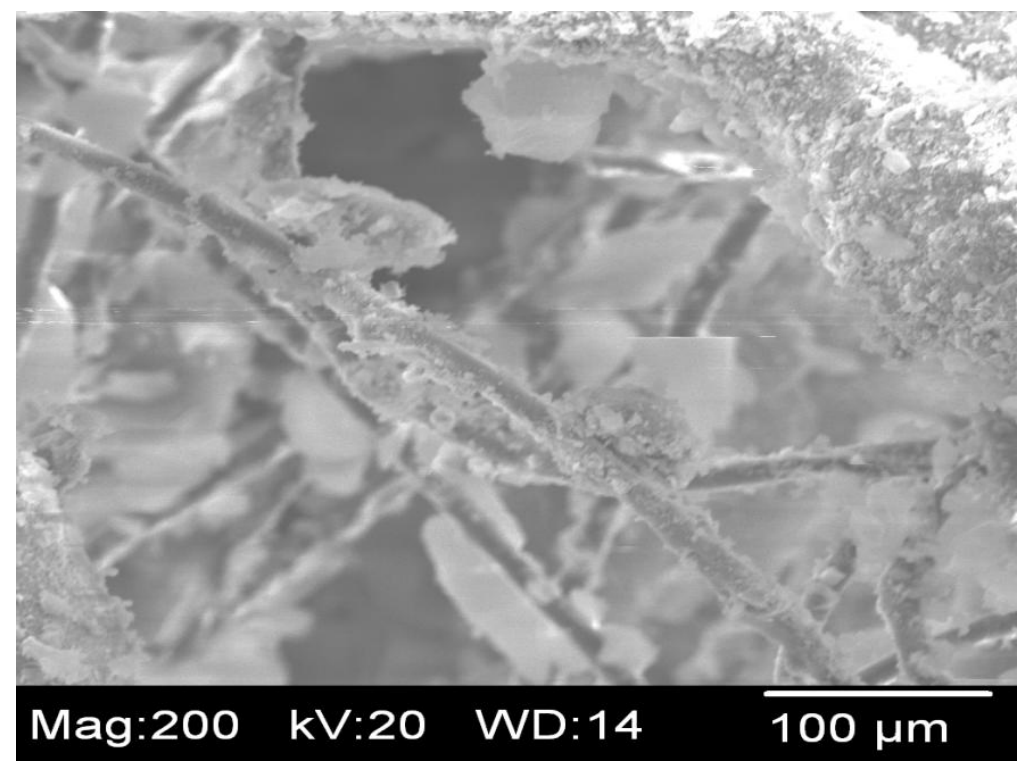

(a)

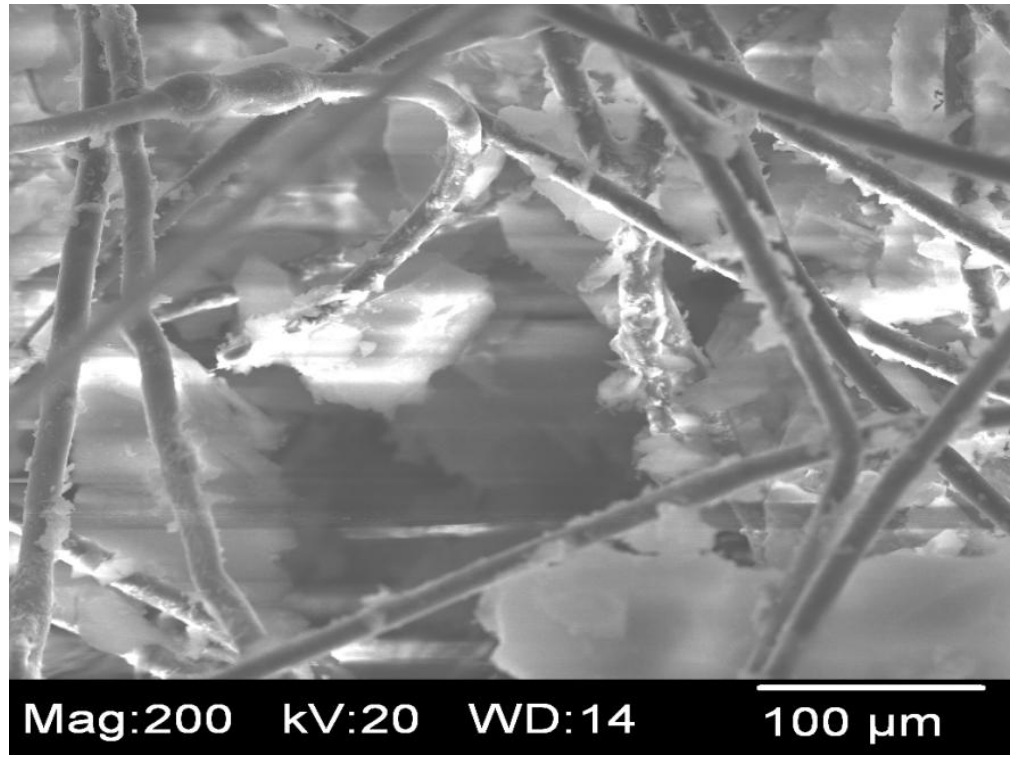

(b)

Figure 4.6: SEM images of tensile Spaceloft material surfaces

(a) AR 3103X; (b) AR 3103 


\subsection{Microscopy Discussions}

SEM results were carried out to exhibit the microstructure and the failure process of Pyrogel and Spaceloft materials.These flexible aerogel blankets are fiber-reinforced composite materials containing fiber-based thermal insulation impregnated with aerogels. The microstructure of aerogel blanket materials is characterized by the existence of many fibers and various aerogel particles of different sizes. In fact, the fibers are surrounded by many aerogels particles. There are also some aerogel particles along the surface of the fibers. The difference between the microstructure of Spaceloft and Pyrogel materials can be explained by factors such as the preparation conditions and the choice of the precursors [60].The average diameter of the fiber for the Spaceloft and the Pyrogel materials is approximately $10 \mu_{m}$. Aerogel networks are formed from open colloidal particles. These particles are interconnected in a highly porous, dendritic- like structure. The structure of aerogel is three dimensional, including many pores, whose diameter ranges from $10 \mathrm{~nm}$ to $15 \mathrm{~nm}$. The fibers are randomly oriented and are incorporated into aerogel blanket matrices. The fibers' contacts points are bonded together. The strength of the blanket materials is determined by the strength of the fibers, the strength of the bonding between the fibers, the strength of the aerogel network and the form of loading. Consequently, the deformation of the fibers will limit the strength of the insulation materials.

In the earliest stage of cracks development, the fibers remain intact. The failure process of fibers usually begins with the development of cracks and the value of crack stress. Indeed, under tension, as the applied stress is increased, more and more cracks will appear in the damaged component aerogel, until finally the fiber component is also 
fractured. During the interval between the appearance of the first crack in the aerogel and the final fracture, a larger strain will be produced by the applied stress. The yield stress of the composite is considered to be the stress at which the first crack appear to the aerogel. The speed of crack propagation will slow when the crack approaches a fiber. This fiber will fail, when a certain value of stress intensity is reached. After this, crack propagation continues to be delayed by subsequent fibers. Fibers prevent the crack propagation in broken blankets throughout the entire composite material. The fibers act as a barrier halting the advance of a crack. Even when there is a complete failure of the insulation blanket, the fibers remain whole and continue to carry the load. The fibers can only fail when the limiting value of deformation of the fibers is reached due to the presence of opening cracks [98-101].

A broken aerogel blanket generates a ductile flow that makes the curved longitudinal fibers of the grid straight. These will not fail and necks will be formed on them. Where there is insufficient bonding strength of the bond between the fibers, there is a partial pulling away from the blanket [102].

The mechanical properties of flexible aerogel blankets can be explained by the transformation of the blanket structure. When the flexible aerogel blankets are characterized or are submitted to mechanical tests, they are susceptible to damage. Damage, in these insulation materials, may occur in the form of micro-cracks and voids. Voids can occur in the matrix of aerogel blanket materials on both sides of a grid. The main reason for the occurrence of a void is the subsequent separation of the grid from the matrix. Voids create a zone of stress concentration, which leads to the failure of a single layer of the blanket. Under mechanical load and weakened coatings, microcracks 
will appear and grow in the fibers until they tear apart and cause the failure of the flexible aerogel blanket [103]. Moreover, the air pocket content increases leading to the reducing the fatigue resistance, increasing the susceptibility to water diffusion and increasing variation in mechanical properties of the blankets.

During the flexural tests, the expansion and contraction rates of the fibers are much different to the aerogel particles. This leads to a disruption of the bonding between the fibers and the aerogel particles and to a premature failure of the blankets. The failure process of aerogel is different from that of the fibers due to the aerogel's porous nature . Compared to most brittle materials, aerogel's structure has more distance between two consecutives bonds that slows down the propagation speed of the stress from one broken bond to another. The pressurized air coming from the broken network of an aerogel will absorb a large amount of the energy from impact. Thus, the speed of failure in aerogel will slow down [104]. The failures of the flexible aerogel blankets are mostly due to accumulation of damage rather than from the growth of a single crack.

The occurrence of cracks in aerogel's structure is determined by the methods used to prepare them. During aerogel fabrication, microcracks may appear in the alcogel and then cracks in the aerogel. Cracking in aerogel depends on the distribution, the shape and the size of the pores of gel and also the chemical linking of sol particles. When cracking starts, a neck forms in the bond between the colloidal particles of aerogel. As the load increases, the neck will grow and the bond between the colloidal particles of aerogel will breakdown. This fracture implies the cleavage of siloxane bonds. With further crack propagation, the pearl string structure of silica beads will also break down. Then, aerogel particles are reduced to sub-micrometer sizes under dispersion conditions. 
When aerogel is fully broken, it changes into micro dust. Fully broken aerogel leads to a macroscopic loss of stiffness and strength in the flexible aerogel blankets [105-107].

\subsection{Flexural Test Results and Discussions}

The results of a three point bending test of Pyrogel and Spaceloft materials are shown in figures 4.7 ( $\mathrm{a}$ and $\mathrm{b}$ ) and 4.8 respectively.

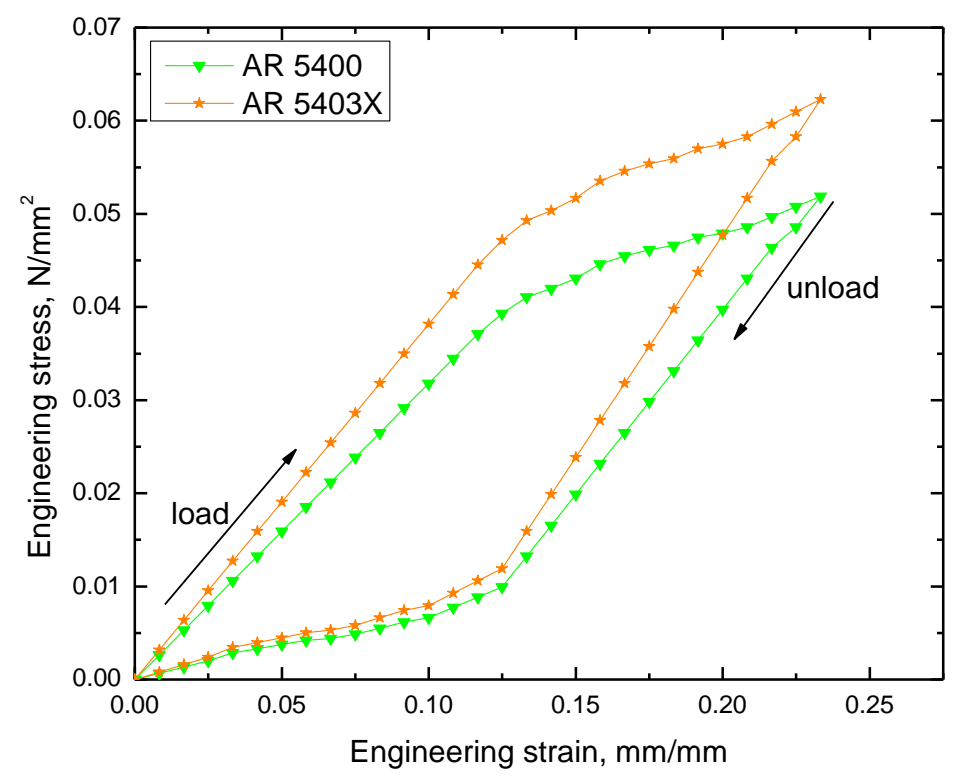

(a) 


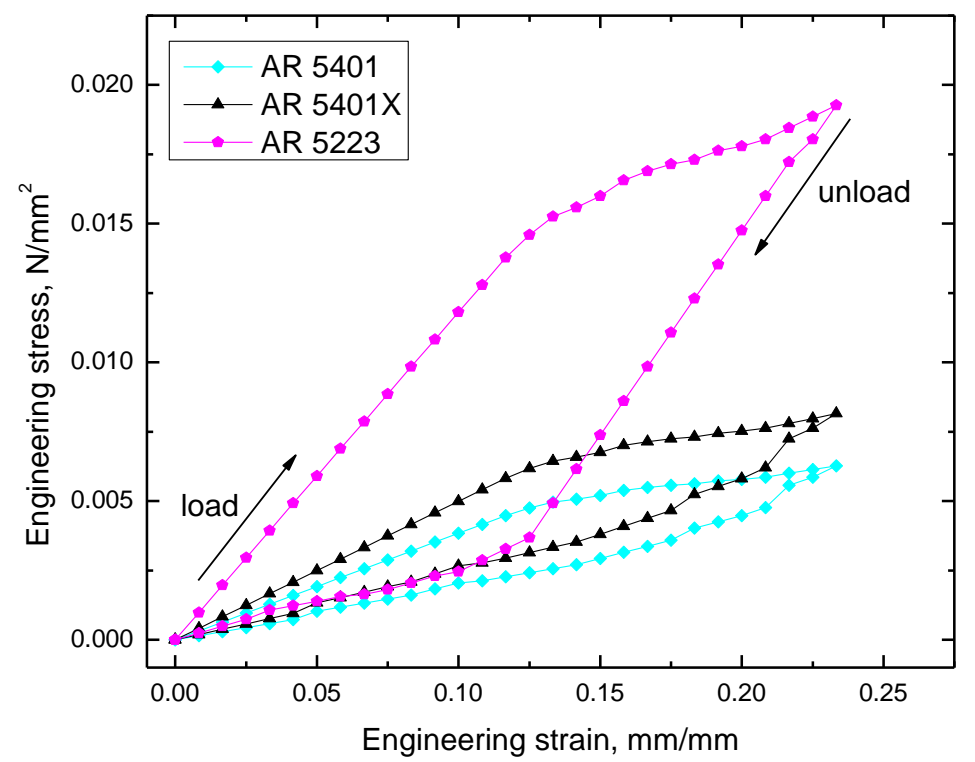

(b)

Figure 4.7: Engineering stress-strain curves of Pyrogel specimens

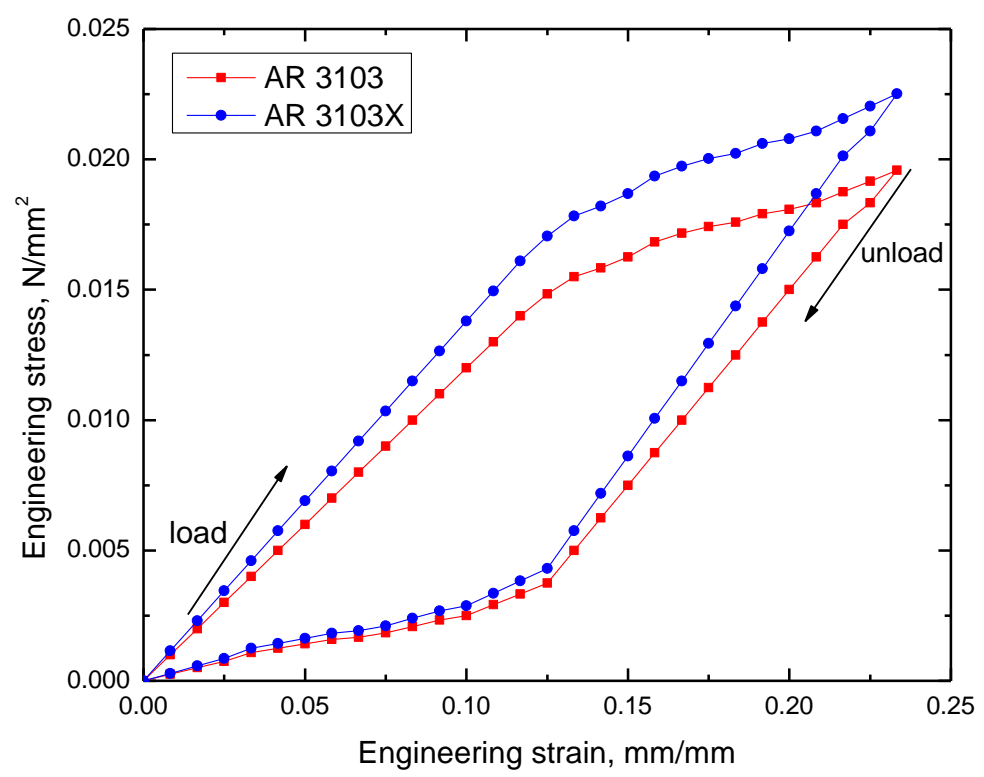

Figure 4.8: Engineering stress-strain curves of Spaceloft specimens

The numerical values of important parameters related to the flexural test of the flexible aerogel blankets are listed in table 4.1 . 
Table 4.1 Three point bending data of testing flexible aerogel blankets

\begin{tabular}{|l|l|l|l|l|l|}
\hline & $\begin{array}{l}\text { Maximum } \\
\text { Load } \\
\text { Materials }\end{array}$ & $\begin{array}{l}\text { Maximum } \\
\text { Bending } \\
\text { Moment } \\
\text { N.mm }\end{array}$ & $\begin{array}{l}\text { Maximum } \\
\text { Stress } \\
\mathrm{N} / \mathrm{mm}^{2}\end{array}$ & $\begin{array}{l}\text { Slope } \\
\mathrm{N} / \mathrm{mm}\end{array}$ & $\begin{array}{l}\text { young's } \\
\text { modulus } \\
\mathrm{N} / \mathrm{mm}^{2}\end{array}$ \\
\hline AR 5400 & 5.29 & 79.35 & 0.051 & 0.529 & 1.359 \\
\hline AR 5403X & 6.23 & 93.45 & 0.06 & 0.623 & 1.453 \\
\hline AR 5223 & 1.92 & 28.8 & 0.018 & 0.0192 & 0.711 \\
\hline AR 5401X & 0.85 & 12.75 & 0.0725 & 0.085 & 0.68 \\
\hline AR 5401 & 0.75 & 11.25 & 0.0675 & 0.075 & 0.60 \\
\hline AR 3103X & 2.70 & 40.5 & 0.0225 & 0.270 & 1.0 \\
\hline AR 3103 & 2.35 & 35.25 & 0.0196 & 0.235 & 0.87 \\
\hline
\end{tabular}

The stress-strain curves consist of a loading portion followed by an unloading one and each portion includes a linear and non-linear part. The linear part of the curve corresponds to a linear elastic deformation and small displacement of the insulation blanket, whereas the non-linear part corresponds to the combined additive effect of material and geometry nonlinearities [108]. During the loading process, there is an increasing flexural load from zero to maximum at maximum displacement. The upper surface of the flexible aerogel blanket is compressed under the loads. The unloading process starts with a decrease in the maximum flexural load to zero and a reverse of the load into tension along the bottom surface of the sample. The flexural strength in compression is about 50\% higher than in tension. A continuous change takes place from maximum tensile stress on one surface through the thickness to maximum compressive stress on the other.

The first cracks always occurred in the center of the specimens and continued to increase with increasing displacements due to the stiffness degradation of the 
specimens. During the loading process, the damage growth in the insulation blankets is characterized by the formation of additional cracks throughout their length.

The load-displacement curves demonstrated a clear dependence of flexural forces on the thickness of aerogel composite materials. The thinner the specimen, the lower the values of corresponding loads in Pyrogel AR 5401/AR 5401X materials. They had the lowest bending strength and lowest stiffness because they were the thinnest. Their bending strength was six and seven times lower than that one of Pyrogel AR 5403X and AR 5400 respectively. For flexible aerogel blankets of equal thickness in the three point bending tests, Spaceloft composites displayed significantly lower flexural loads for failure and lower bending moment values than Pyrogel AR 5400 and AR 5403X.

Another criterion used to evaluate the mechanical performance of the flexible aerogel blanket was the area under the load-displacement curve. This area defined the energy required to fracture the critical cross section under the load point. Pyrogel AR 5400 and AR 5403X exhibited the highest bending energy.

To quantify the dusting process during the flexural test, the curves of mass loss of the Pyrogel and Spaceloft specimens were plotted in figure $4.9(a, b)$ and figure 4.10 respectively. 


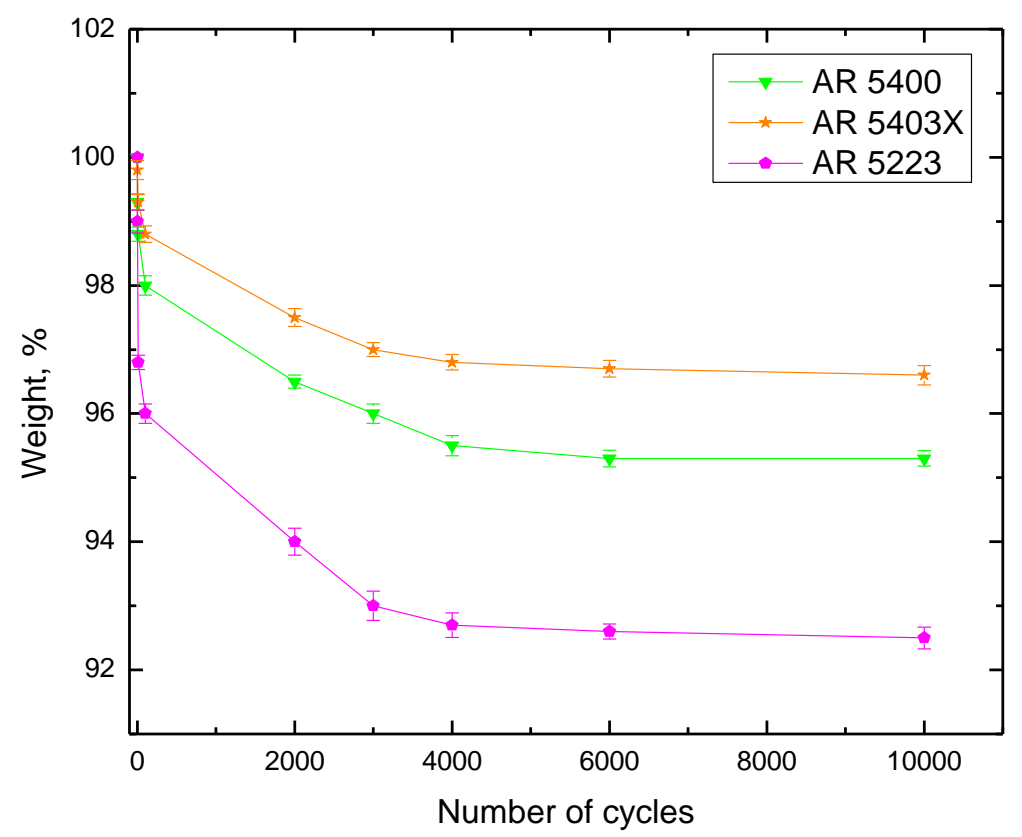

(a)

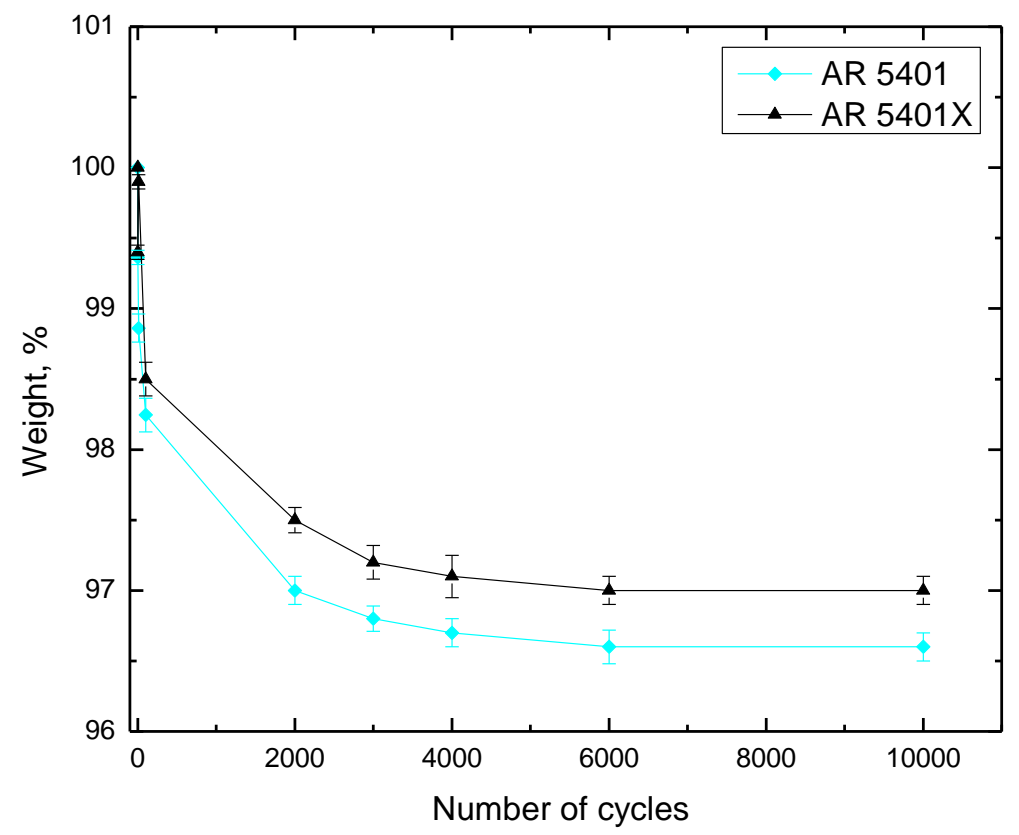

(b)

Figure 4.9: Graph of weight loss versus cycles for Pyrogel specimens 


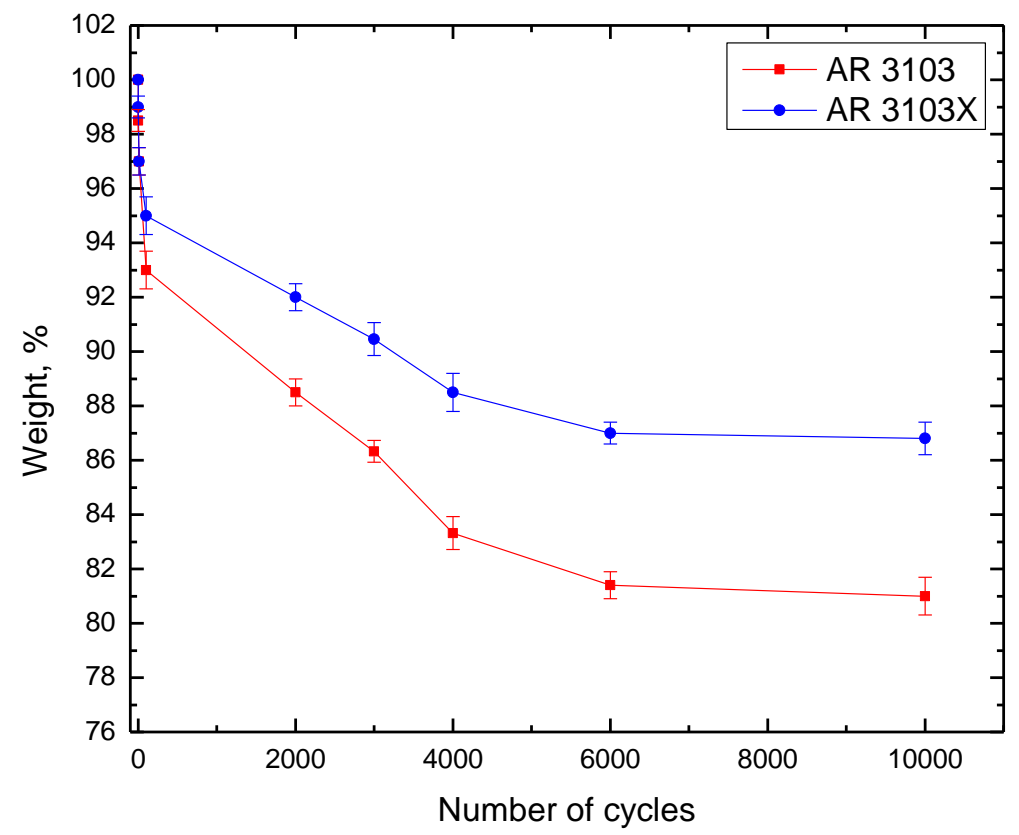

Figure 4.10: Graph of weight loss versus cycles for Spaceloft specimens

The trends in the variation of the mass loss for three point bending test exhibited similar behavior for all flexible aerogel blankets. Increase in the rate of weight loss in line with increased cycles can be attributed to the increased rate of stress concentration on the surface of the blankets. In fact, during the repeated load process, the stress concentration caused deformation leading to a small crack in the blankets. When a sample is loaded in the same way multiples times, eventually, the crack will grow [109].

Different damage mechanisms occurred during cyclic loading including aerogel cracking, fiber fracture, fiber/aerogel debonding and delamination. The first damage mechanism is aerogel cracking initiating in the zone free of fibers. As the number of cycles increase, the second and third damage mechanisms occur which are debonding at the fiber aerogel interface and delamination between the layers respectively. The final damage stage is the failure of fibers. 
Although Pyrogel samples AR 5401X and AR 5401 had less flexural strength than Pyrogel AR 5400, AR 5223 and AR 5403X, their mass loss was small because of their thinness. With Pyrogel and Spaceloft specimens having the same thickness, their weight losses were proportional to their flexural strength. In a more explicit way, Spaceloft AR 3103 has three times less flexural strength than Pyrogel AR 5400 and consequently will show approximately $15 \%$ mass loss more.

The materials with coated surfaces lost less weight than their counterparts. Pyrogel AR 5401X lost an average of $0.5 \%$ less than Pyrogel AR 5401. After 10000 cycles of loading, Pyrogel AR 5400 retained almost $97 \%$ of its weight. Among the flexible aerogel blanket materials tested, Spaceloft AR 3103 lost the most weight. The maximum mass loss in this experiment was more than 19\%. The average difference between Pyrogel AR 5400 and Pyrogel AR 5223 was 5\%. This confirmed that the mechanical loading of the insulation blankets had a very significant effect on their dusting process.

\subsection{Tensile Test Results and Discussions}

The engineering stress and engineering strain of Pyrogel and Spaceloft materials obtained under tensile load are plotted in figure 4.11 and figure 4.12 


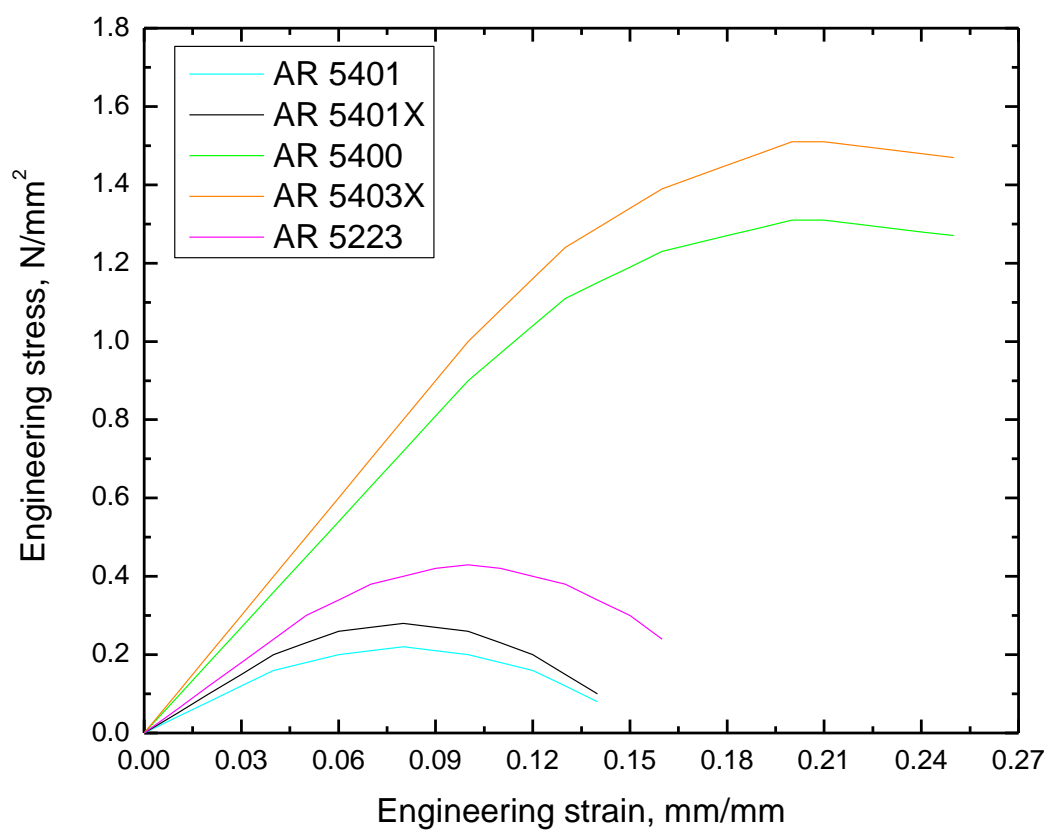

Figure 4.11: Engineering stress-strain curves of Pyrogel specimens

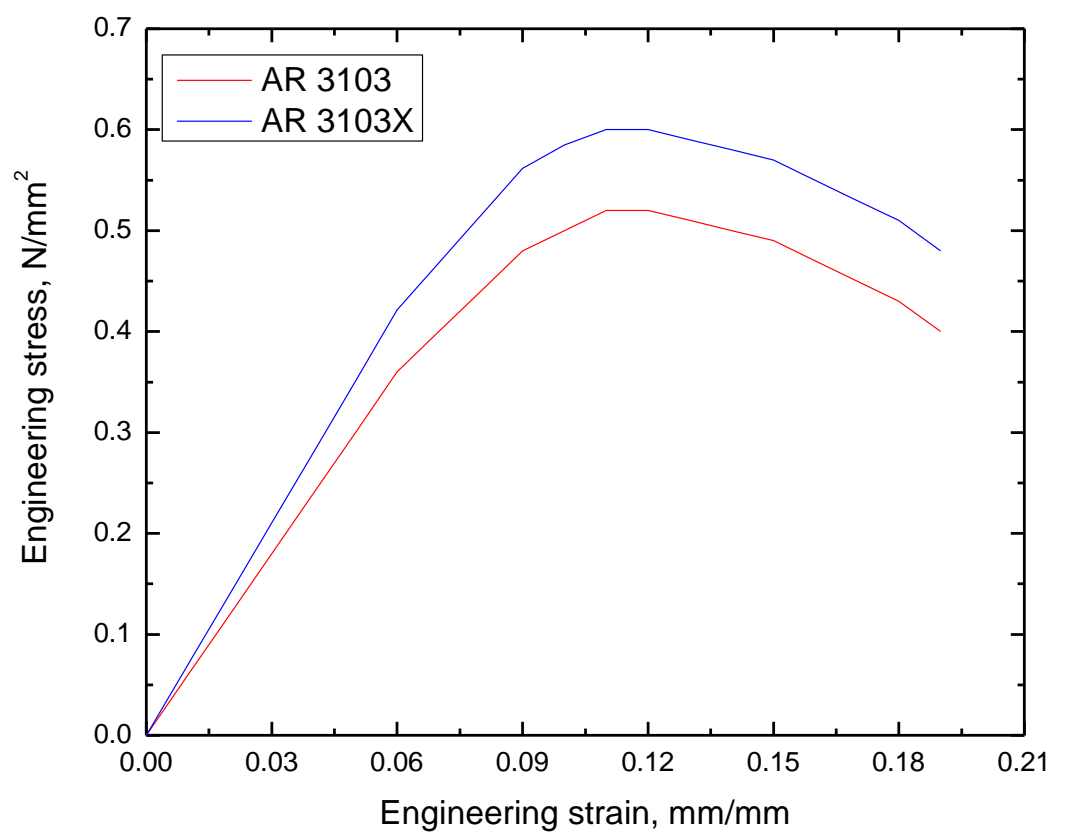

Figure 4.12: Engineering stress-strains curves of Spaceloft specimens 
Data from tensile test were used to determine the parameters shown in table 4.2

Table 4.2 Tensile data of testing flexible aerogel blankets

\begin{tabular}{|c|c|c|c|c|c|c|c|}
\hline Materials & 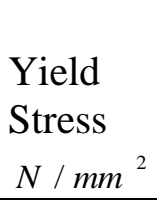 & $\begin{array}{l}\text { Yield } \\
\text { Strain } \\
\mathrm{mm} / \mathrm{mm}\end{array}$ & $\begin{array}{l}\text { Young's } \\
\text { modulus } \\
\mathrm{N} / \mathrm{mm}^{2}\end{array}$ & $\begin{array}{l}\text { Ultimate } \\
\text { Tensile } \\
\text { Stress } \\
N / \mathrm{mm}^{2}\end{array}$ & $\begin{array}{l}\text { Ultimate } \\
\text { Tensile } \\
\text { Strain } \\
\mathrm{mm} / \mathrm{mm}\end{array}$ & $\begin{array}{l}\text { Rupture } \\
\text { Stress } \\
\mathrm{N} / \mathrm{mm}^{2}\end{array}$ & $\begin{array}{l}\text { Rupture } \\
\text { Strain } \\
\mathrm{mm} / \mathrm{mm}\end{array}$ \\
\hline AR 5400 & 1.10 & 0.11 & 10 & 1.26 & 0.20 & 1.2 & 0.25 \\
\hline AR 5403X & 1.18 & 0.10 & 11.8 & 1.5 & 0.20 & 1.4 & 0.25 \\
\hline AR 5223 & 0.32 & 0.05 & 6.4 & 0.4 & 0.1 & 0.5 & 0.28 \\
\hline AR 5401 & 0.19 & 0.045 & 4.22 & 0.25 & 0.07 & 0.10 & 0.13 \\
\hline AR 5401X & 0.20 & 0.045 & 4.44 & 0.28 & 0.07 & 0.12 & 0.13 \\
\hline AR 3103 & 0.35 & 0.055 & 6.36 & 0.48 & 0.12 & 0.38 & 0.19 \\
\hline AR 3103X & 0.38 & 0.055 & 6.91 & 0.58 & 0.12 & 0.5 & 0.19 \\
\hline
\end{tabular}

The engineering stress-strain curves of the flexible aerogel blanket materials can be divided into Four (4) regions. The first region is the elastic region where the material returned to its original length when the load was removed. It is defined as an area between the origin point and the elastic limit point. This region is typically represented by a linear relationship between the stress and the strain. The Young's modulus was used to define the slope of the straight line portion of the stress-strain diagram. Comparing the Spaceloft materials, one can see that AR 3103X had higher stiffness than AR 3103 since its elastic modulus was about 13\% higher. Pyrogel materials AR 5400 and AR $5403 \mathrm{X}$ had the highest stiffness based on the value of their modulus.

The second region is the strain hardening region, which was defined between the yield point and the ultimate strength. In this region, stress increased until it reached its maximum value. The Ultimate Tensile Stress (UTS) of AR 3103X was 16\% more than that of AR 3103. Pyrogel materials AR 5400 and AR 5403 X also had approximately four 
and five times the tensile strength of Pyrogel materials AR 5223 and AR 5401 respectively at a strain of $0.20 \mathrm{~mm} / \mathrm{mm}$.

After passing the UTS, the cross-sectional area of the tested flexible blankets decreased because of the presence of plastic flows. Consequently, the engineering stress decreased slowly and moved toward a limit value. The insulation material underwent a period of necking, in which it broke down. During the necking period, the values of stress dropped in line with increasing strain value. The flexible aerogel blanket materials withstood very low strains before the specimen rupture. The last region occurred when a flexible aerogel blanket had broken into two separate parts.

The fracture energy or the energy absorbed (per unit volume) of the flexible aerogel blankets was defined by the area under the stress-strain curves. Spaceloft AR 3103X had greater fracture energy than AR 3103 and AR 5401 had the lowest of the Pyrogel samples.

Under the action of tensile load, the aerogel composites were extended and the dimensions of cracks became larger [110]. Then, failure began with a peak retentive value followed by a rapid decrease in stress. The mechanical measurements under tensile loads showed that Pyrogel materials AR 5400 and AR 5403 X decayed at a much slower rate than Spaceloft materials AR 3103 and AR 3103X, due mainly to their physical nature and chemical composition.

ASTM D30369 (1995) [111] was used to describe and identify the failure modes, areas and locations of flexible aerogel blankets under tension. Three different types of failure modes and locations were observed during the tensile test of different blanket specimens. Some specimens failed as lateral, others as angled while the remaining failed 
as middle. For the first group of failure, the fracture occurred at one side of the specimen and then moved to the other side across the width. For the second group of failure, the failure occurred in the area close to the center of the samples. For the last group of failure type, the failure was in the middle of the specimens and a line perpendicular to the longitidunal direction was used to describe the fracture. Consequently, the specimens are divided in two equal parts and the failure is sudden and catastrophic.

During the tensile test, when the Pyrogel samples were expanded at a constant rate, they lost their mass. Figures 4.13 and 4.14 exhibit the mass loss of the Pyrogel and Spaceloft specimens under tensile load respectively.

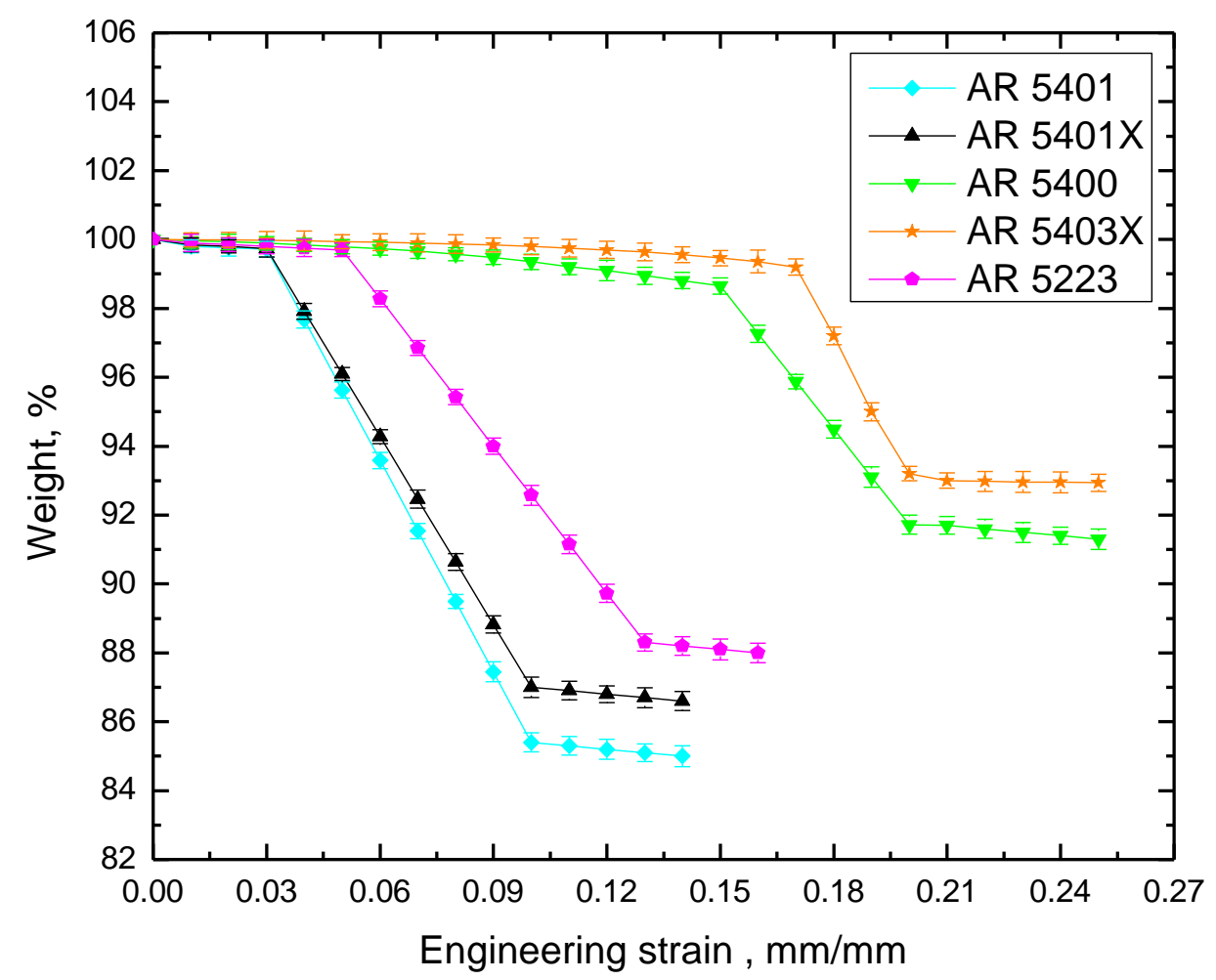

Figure 4.13: Graph of weight loss versus displacement of Pyrogel specimens 


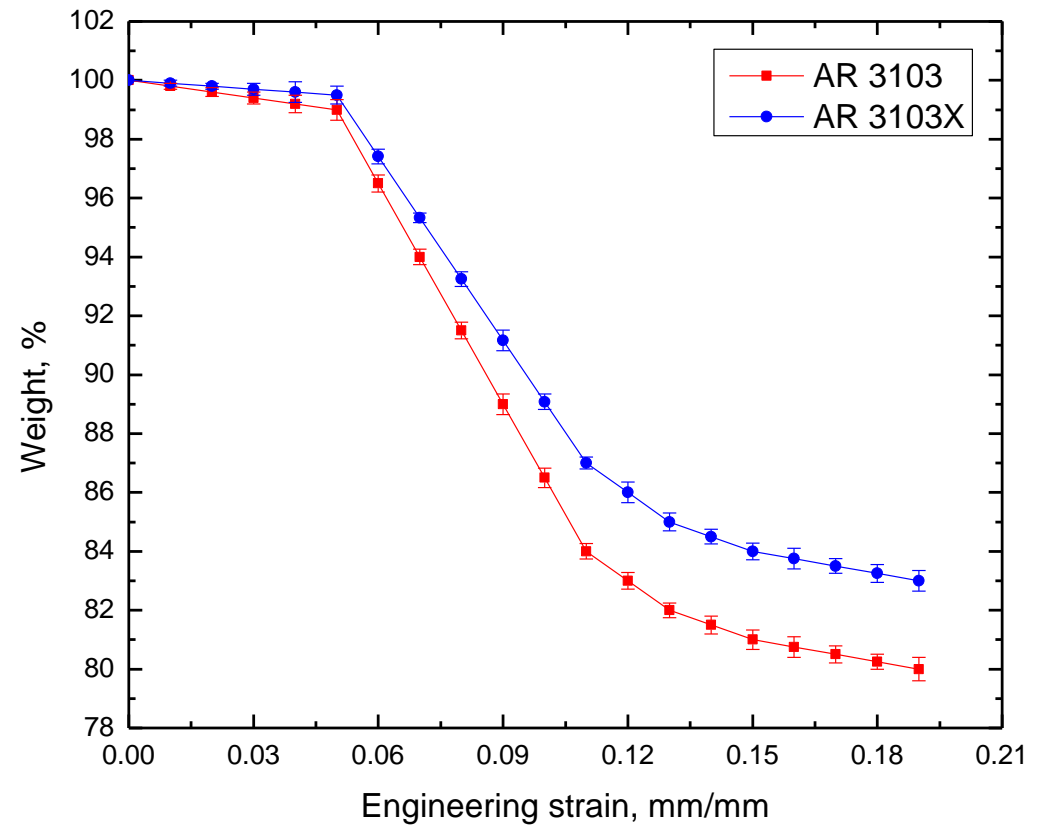

Figure 4.14: Graph of weight loss versus displacement of Spaceloft specimens

Similar to the flexural test, Pyrogel AR 5223 and Spaceloft AR 3103 lost more weight than the others. One can see that the reduction in the blankets' mass was higher under tensile load than the flexural test. The difference of mass loss for Pyrogel AR 5223 and Spaceloft AR 3103 during the tensile and flexural tests were $8 \%$ and $10 \%$ respectively. The blanket samples were more damaged during the tensile test than the flexural one.

\subsection{Results of Thermal Resistance Measurements}

Thermal measurements data collected at $125^{\circ} \mathrm{C}$ for Pyrogel and Spaceloft blanket material specimens are shown in figures 4.15 and 4.16 respectively. 


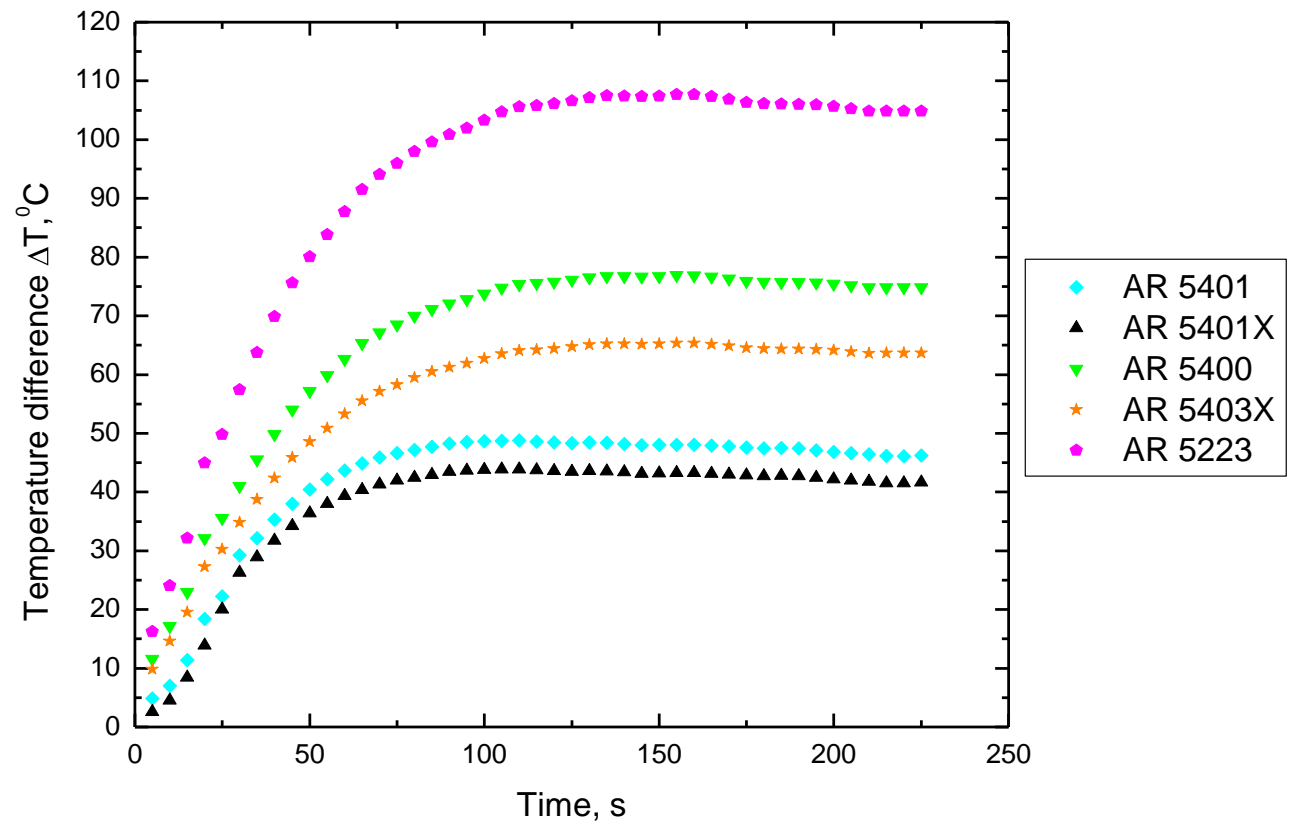

Figure 4.15: Test of Pyrogel blankets at $125^{\circ} \mathrm{C}$

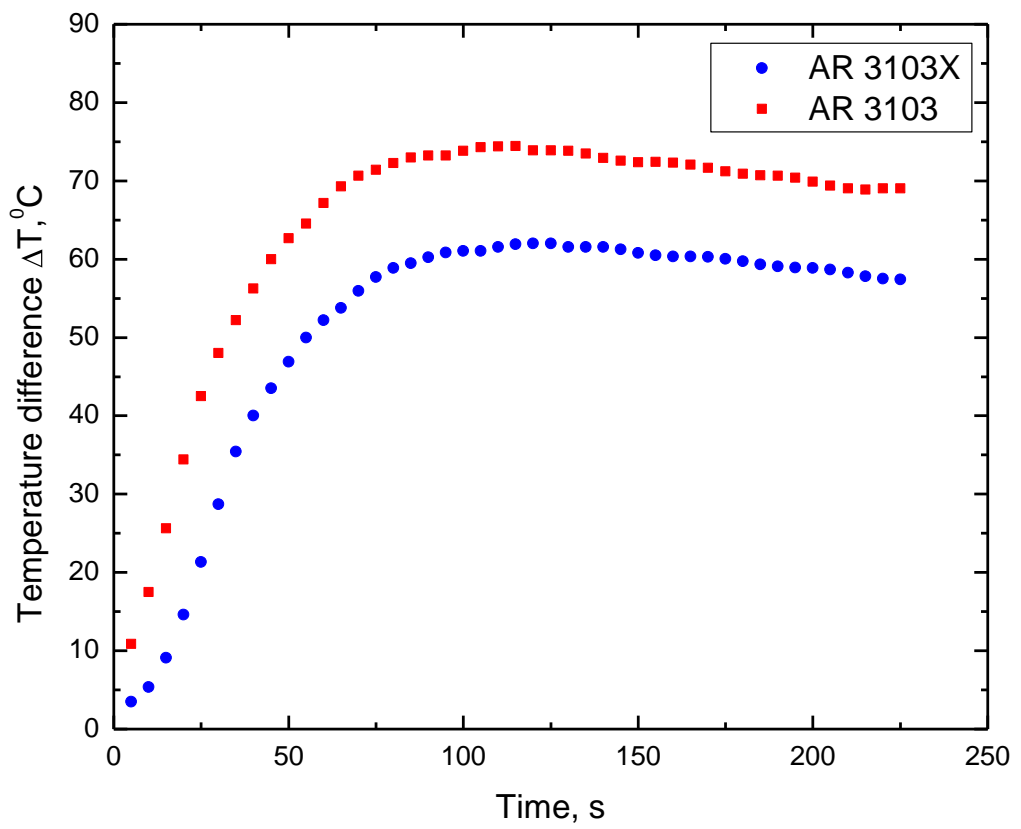

Figure 4.16: Test of Spaceloft blankets at $125^{\circ} \mathrm{C}$ 
When the hot plate was set up to $125^{\circ} \mathrm{C}$, its temperature was equalled to the room temperature, $25^{\circ} \mathrm{C}$, and reached the selected temperature within 225 seconds. It is interesting to note that the temperature differences across the samples increased as the temperature of the hot plate increased. The values of the temperature difference across the insulation materials were proportional to the thickness and the chemical composition of their material surfaces. The smallest temperature difference across the samples were obtained for Pyrogels AR 5401 and AR5401X because of their thinness. The values of the temperature difference across the Pyrogel and Spaceloft materials with coated surfaces were lower than those with a non- coated surface.

The steady state temperature for top surface of the flexible aerogel blankets, at six different temperatures of the hot plate, are shown in figures 4.17 and 4.18.

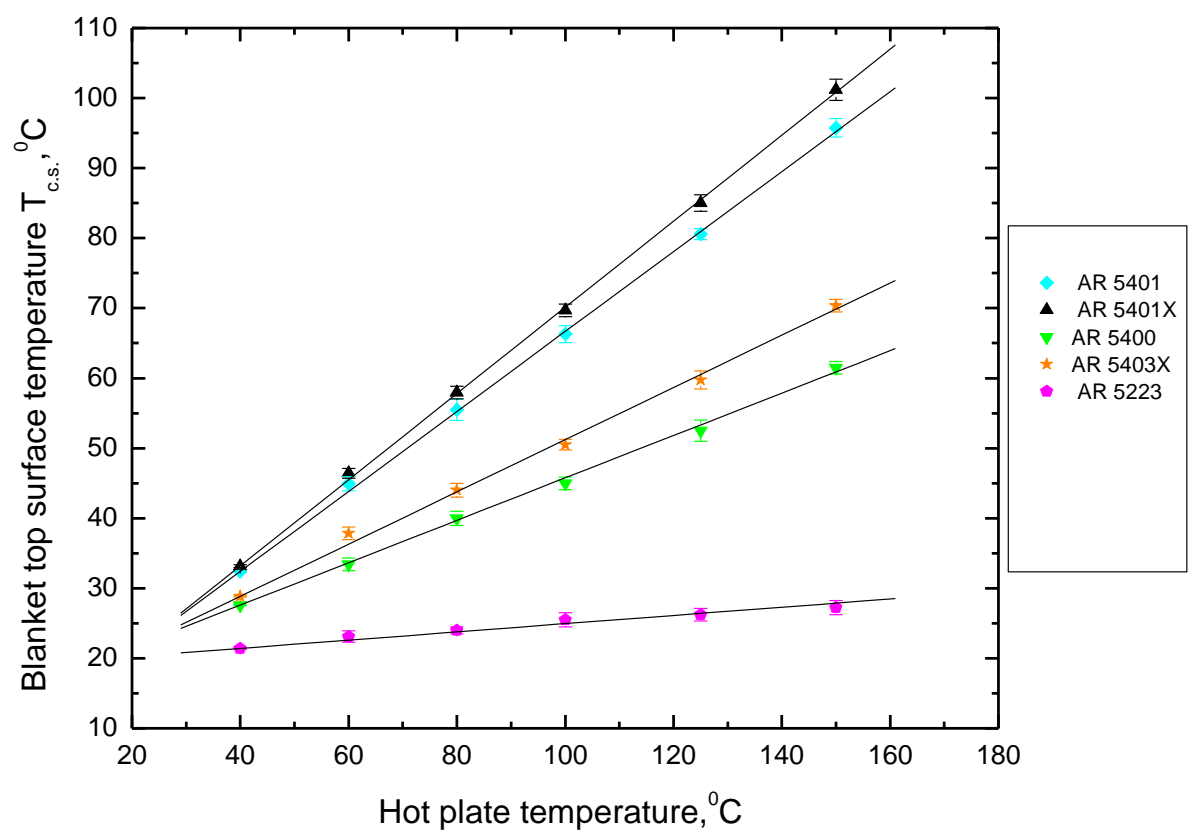

Figure 4.17: Thermal test at steady temperatures of Pyrogel blankets 


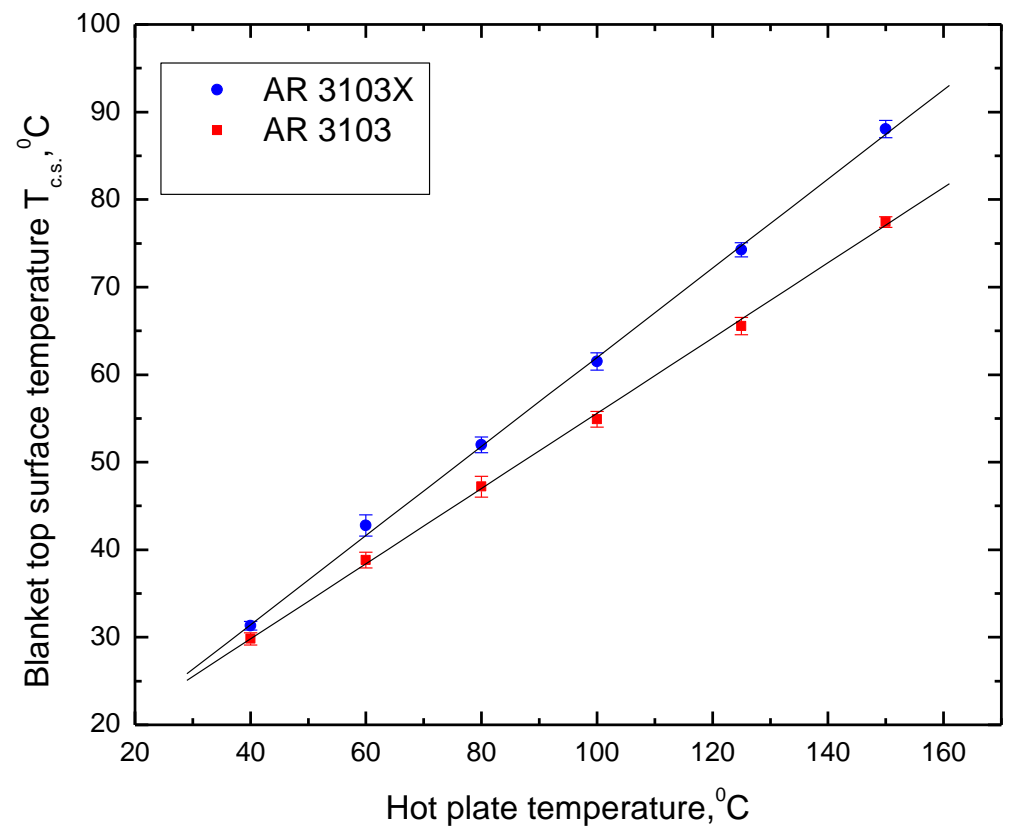

Figure 4.18: Thermal test at steady temperatures of Spaceloft blankets

The temperature of the top surface of all the flexible aerogel blankets increase linearly with increasing temperature of the hot plate. It has been considered that the blankets with the lowest top surface temperature have the highest thermal resistance and the highest thermal insulation. Pyrogel blanket AR 5223 exhibits the highest thermal resistance and insulation whereas Pyrogel blanket AR 5401/5401X exhibit the lowest thermal resistance and insulation due to their thinness. The heat flow reachs much faster the surface temperature of blanket materials with smaller thickness and small number of aerogel layers.

Using equation 3.5, the results of the normalized relative temperature drop of the flexible aerogel blanket for different temperatures of the hot plate are plotted in figure 4.19 and 4.20 . 


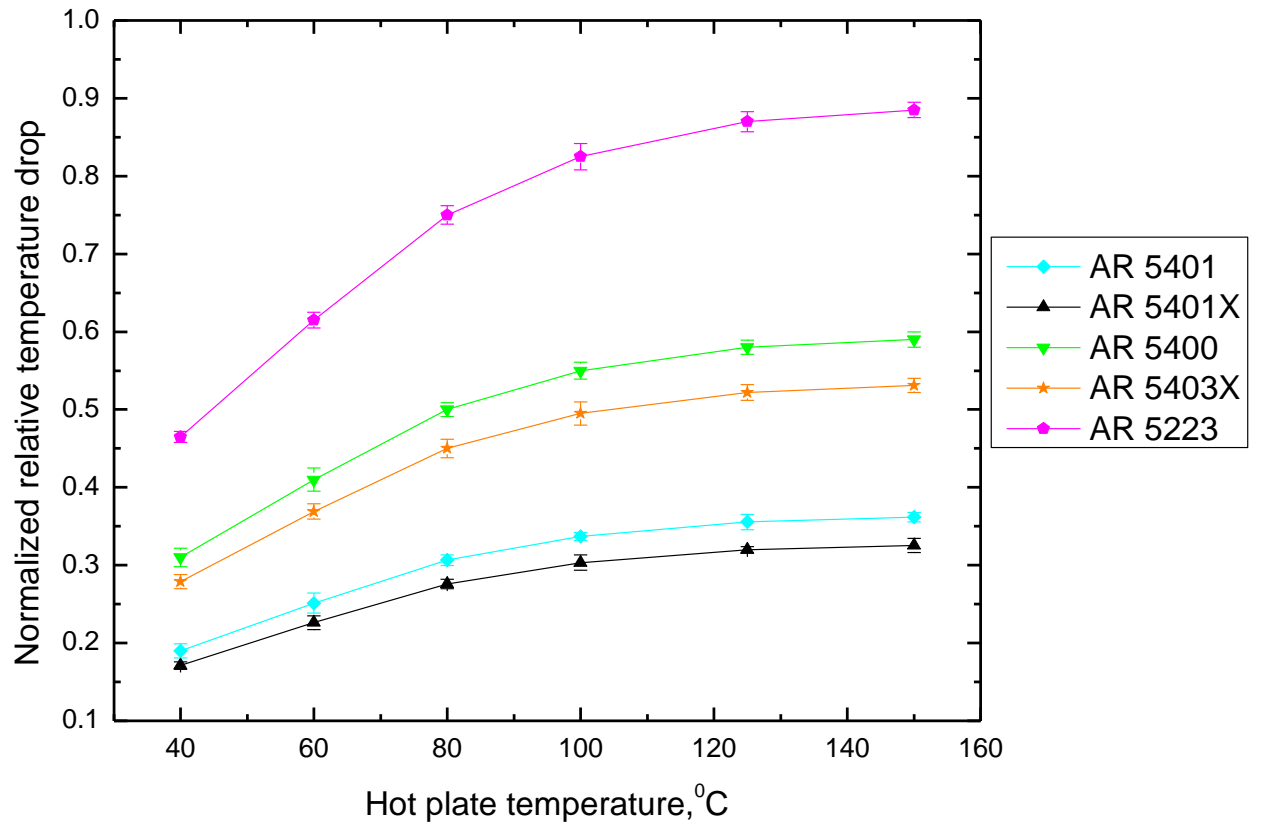

Figure 4.19: Normalized relative temperature drop of Pyrogel blankets

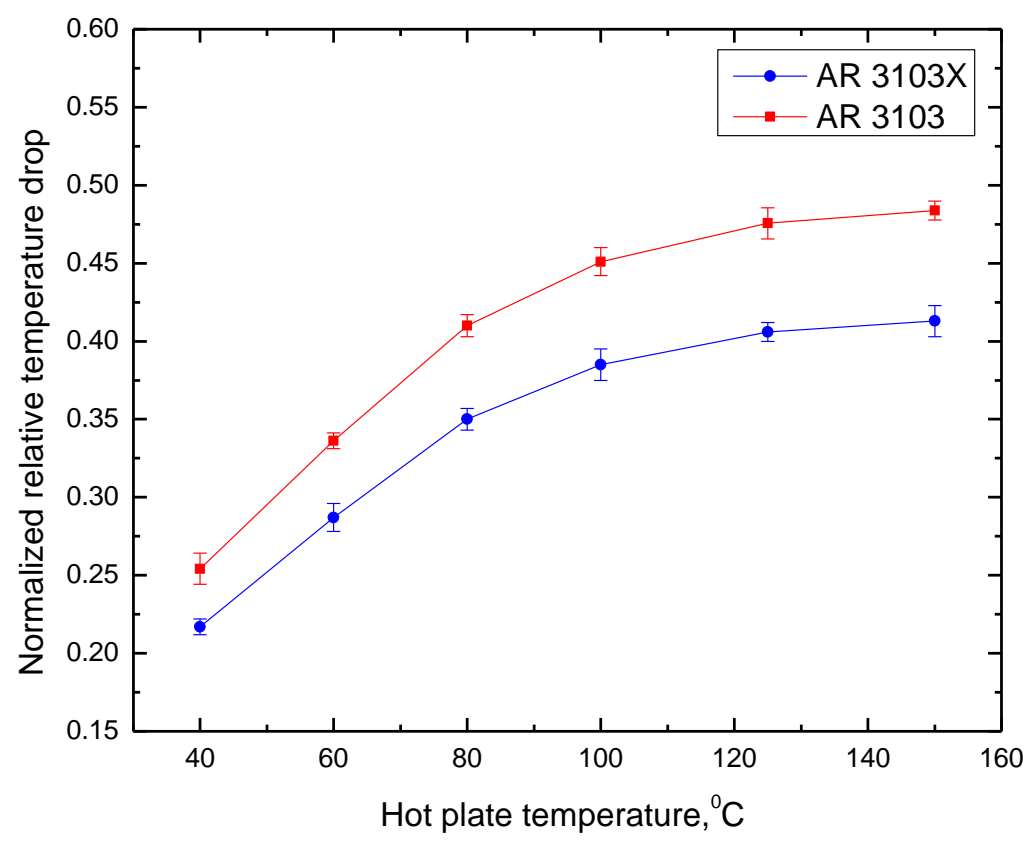

Figure 4.20: Normalized relative temperature drop of Spaceloft blankets 
The relative normalized drop temperature reached its highest value at higher hot plate temperature. Both Pyrogel and Spaceloft materials show higher thermal performance at higher hotplate temperature. Pyrogel AR 5401/5401X had the lowest normalized relative temperature drop because of their thicker geometry. Comparing flexible aerogel blankets of the same thickness, Pyrogel materials exhibit higher normalized relative temperature drop than Spaceloft with an average of approximately 10\%. So, Pyrogel can be used for high temperature applications whereas Spaceloft materials are more suitable for low temperature applications.

The temperature difference between the top and the bottom surfaces of flexible aerogel blankets when undergoing a three point bending test are shown in figure $4.21(\mathrm{a}$ and $\mathrm{b})$ and 4.22 .

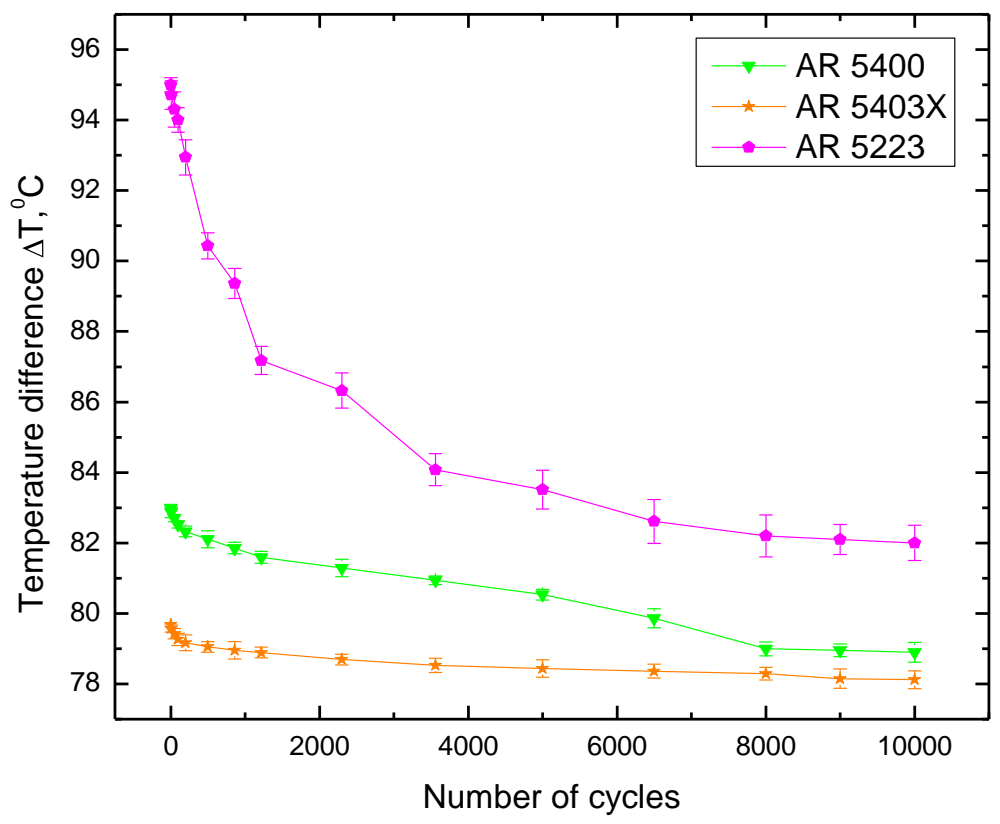

(a) 


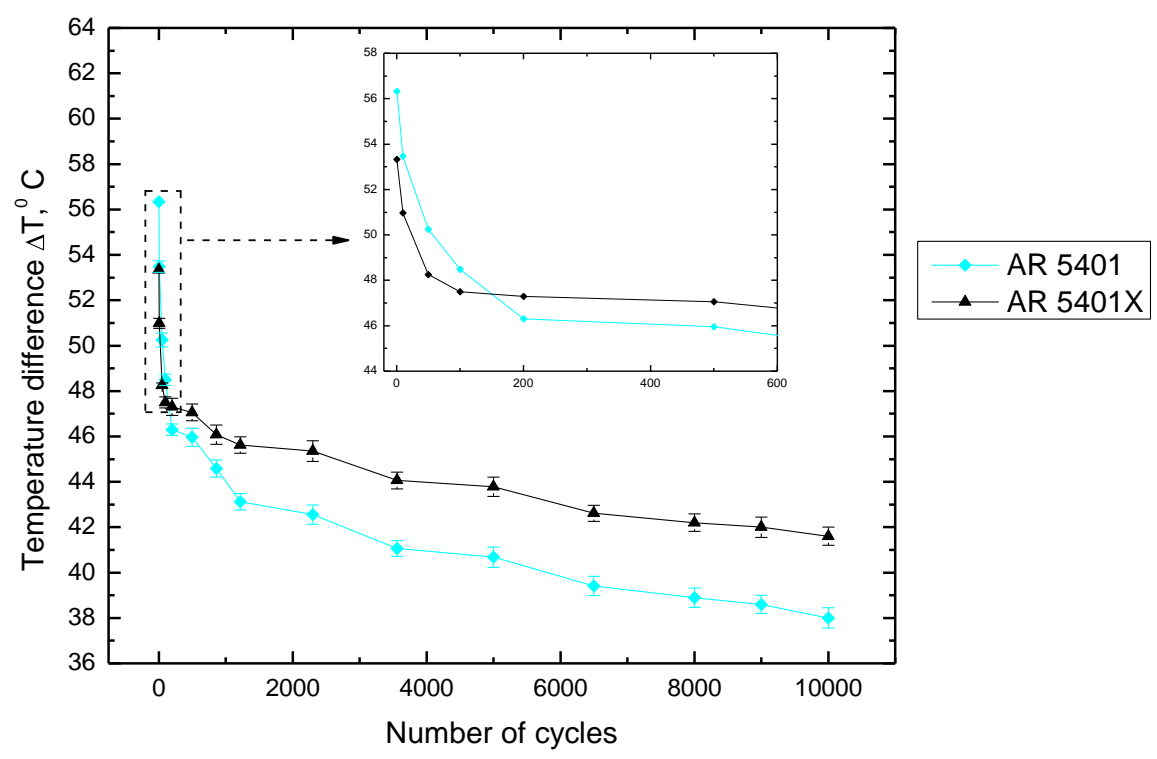

(b)

Figure 4.21: Temperature difference across Pyrogel blankets at $T_{\text {hotplate }}=150{ }^{\circ} \mathrm{C}$ under the flexural test

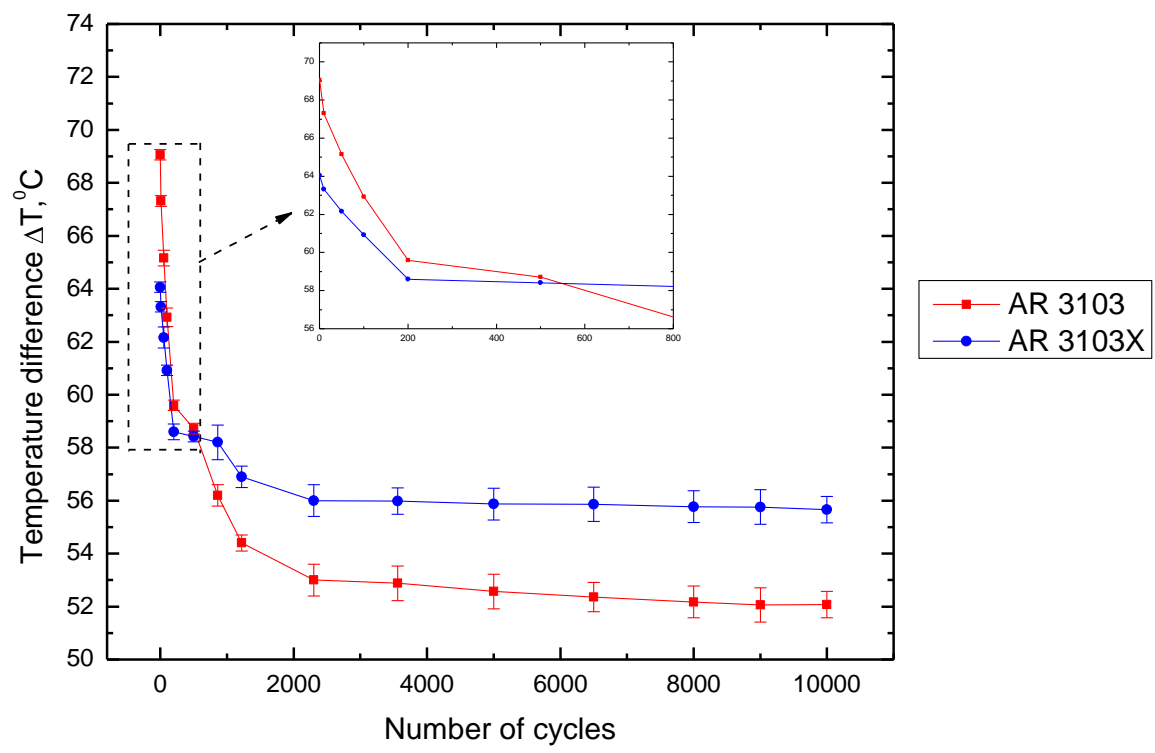

Figure 4.22: Temperature difference across Spaceloft blankets at $T_{\text {hotplate }}=150{ }^{\circ} \mathrm{C}$ under the flexural test 
Figures 4.23 and 4.24 illustrate the temperature differences across the insulation blankets associated with the displacements measurements along the specimens when a tensile load was applied to the flexible aerogel blanket.

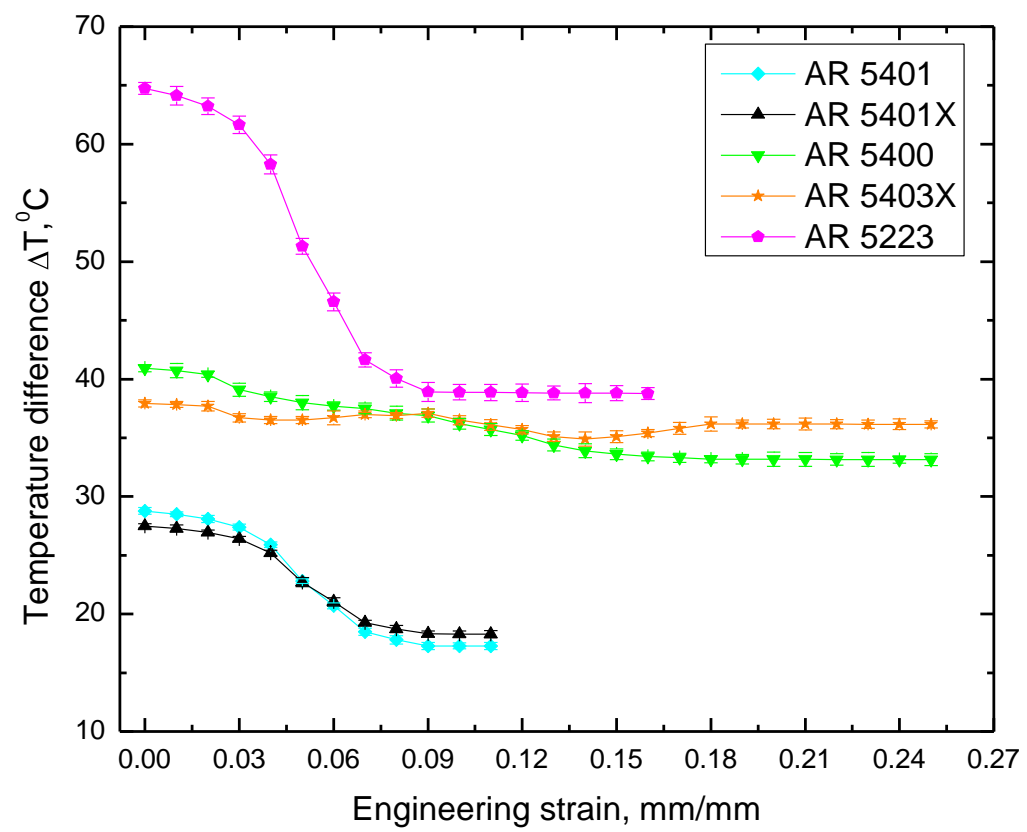

Figure 4.23: Temperature difference across Pyrogel blankets at $T_{\text {hotplate }}=75^{\circ} \mathrm{C}$ under tensile test 


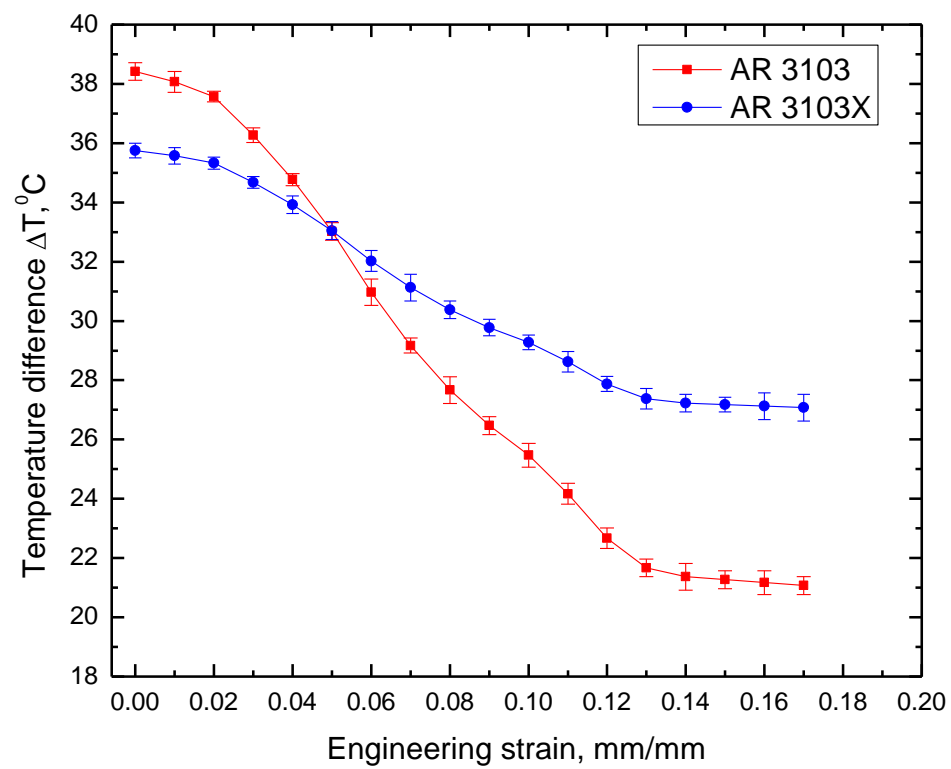

Figure 4.24: Temperature difference across Spaceloft blankets at $T_{\text {hotplate }}=75^{\circ} \mathrm{C}$ under tensile test

These figures show the effect of the mechanical loading of flexible aerogel blankets on their thermal insulation performance. The temperature differences across the surfaces of the flexible aerogel blankets decreased gradually with either the increasing number of cycles during the flexural test or the increasing crosshead displacement under tensile loads until a stabilized value was reached. The stabilized value of the temperature differences corresponded in the tensile test to the displacement where UTS value of the sample was reached and in the three point bending test to the permanent deformation of the sample. The temperature differences were higher in tensile test than in three- point bending tests. These results indicate higher degradation of thermal properties of aerogel insulation blankets under tensile tests. Pyrogel AR 5223 and Spaceloft AR 3103 showed a reduction of their temperature difference by $10^{\circ} \mathrm{C}$ and $17^{\circ} \mathrm{C}$ respectively after 10000 cycles during the flexural test and by $25{ }^{\circ} \mathrm{C}$ and $18{ }^{\circ} \mathrm{C}$ respectively during the 
tensile test. There was also a difference in temperature changes between coated and noncoated materials. At lower values of displacement or cycles, the non-coated flexible aerogel blanket AR 3103 and AR 5401 showed higher thermal performance than the coated AR 3103X and AR 5401X but at higher displacement and cycle values, the thermal performance of non-coated blankets was smaller. The coating helped the blanket surfaces to resist material degradation, although its presence initially reduced their thermal properties. Pyrogel AR 5401/5401X exhibited the lowest temperature difference during the mechanical testing because of their thinness. In general, Pyrogel materials showed higher thermal resistance than Spaceloft materials. Of all flexible aerogel blankets, Pyrogel materials AR 5400 and AR 5403X had considerable resistance to mechanical degradation and keept their thermal properties best.

\subsection{TGA results and discussions}

The thermal stability and weight loss rates of the flexible aerogel blanket materials were evaluated with TGA. Figures 4.25 and 4.26 showed the TGA 
thermograms of the investigated aerogel blanket materials heated under air.

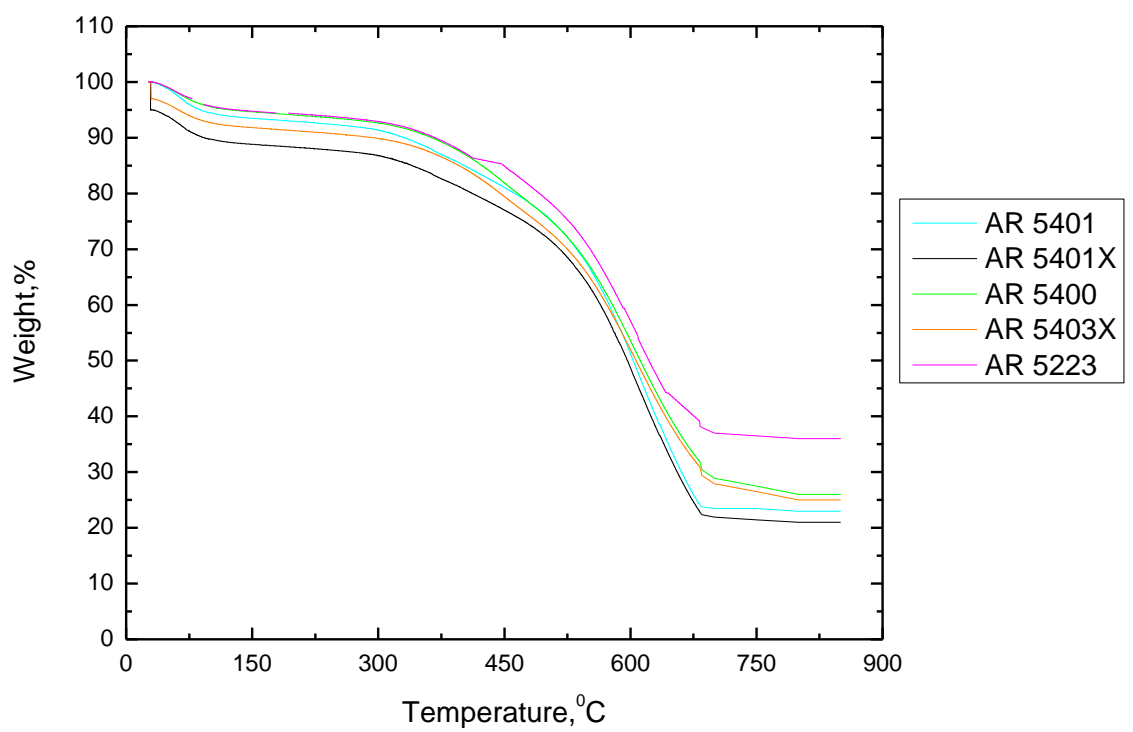

Figure 4.25: TGA thermograms of Pyrogel blanket materials in air

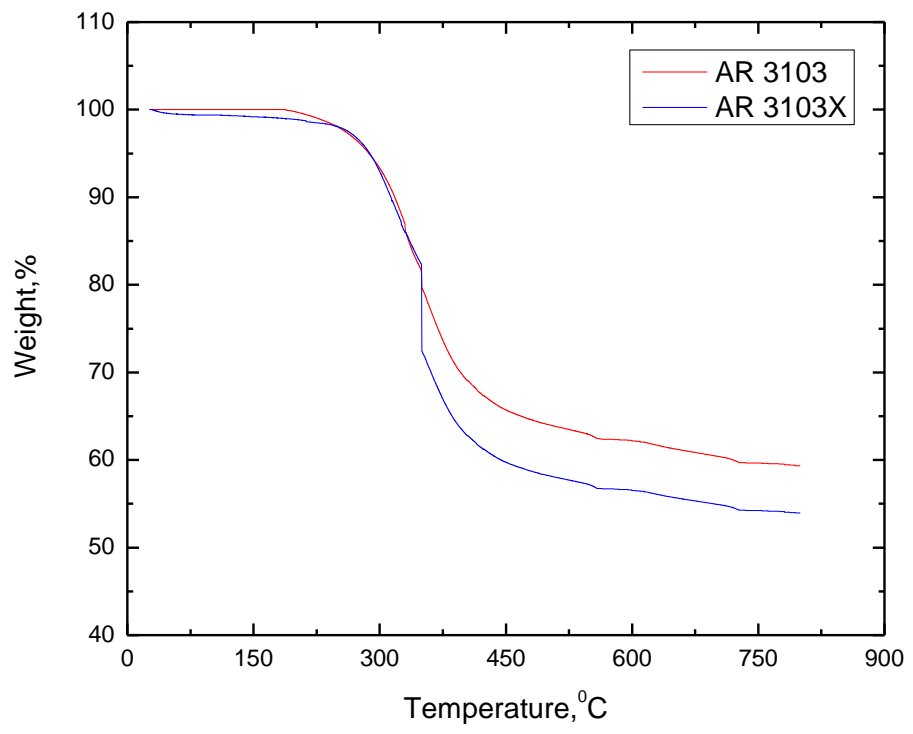

Figure 4.26: TGA thermograms of Spaceloft blanket materials in air

TGA thermograms of the investigated aerogel blanket materials heated under nitrogen are shown in figure 4.27 and 4.28 . 


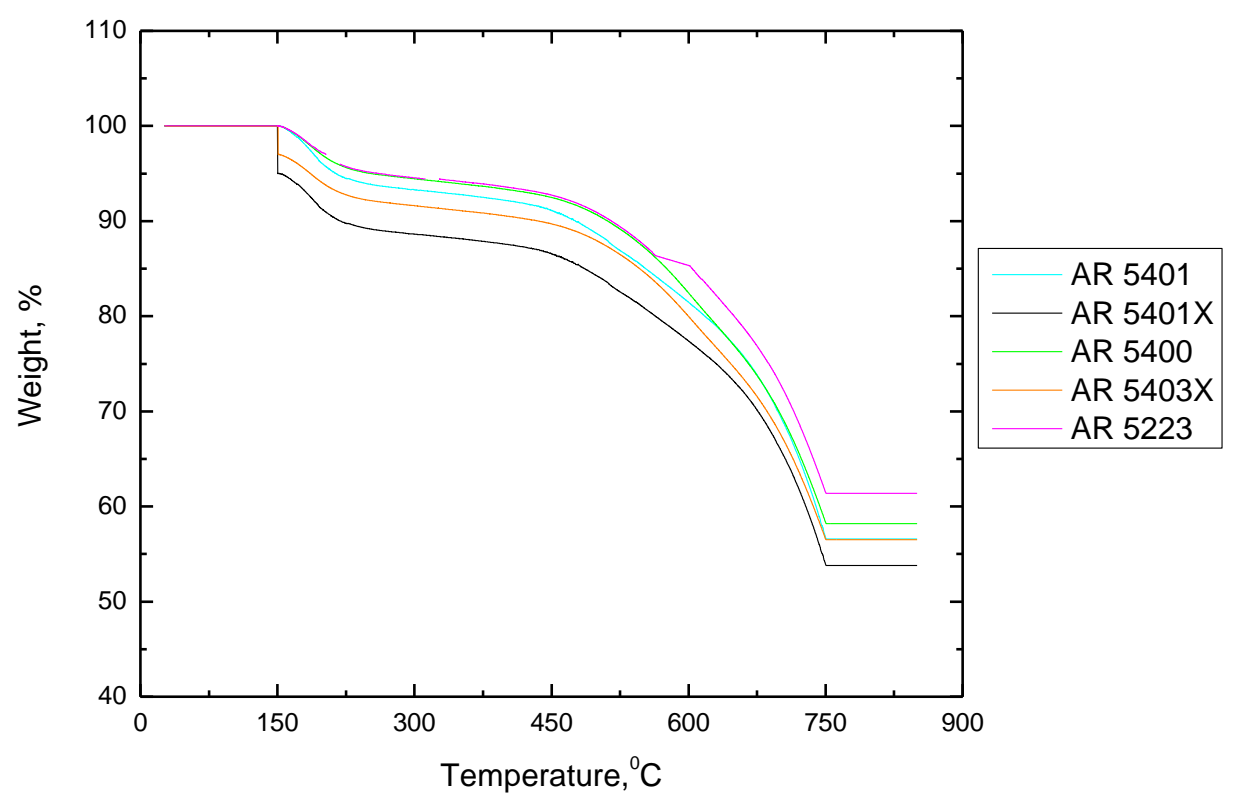

Figure 4.27: TGA thermograms of Pyrogel blanket materials in nitrogen

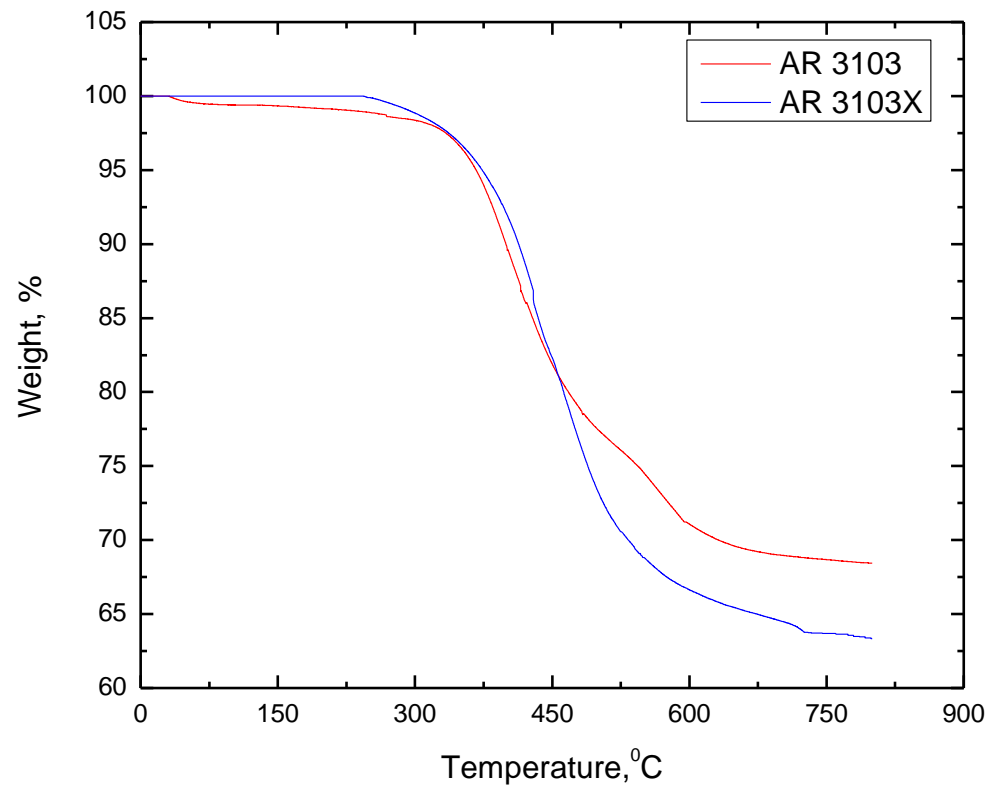

Figure 4.28: TGA thermograms of Spaceloft blanket materials in nitrogen 
From figures 4.26-4.28, it can be seen that the loss of weight of flexible aerogel blankets is continuously decreasing as the temperature is raised at constant rate under atmospheric air and nitrogen. All the blankets display three steps decomposition behavior under air and nitrogen. For Spaceloft materials, in air and nitrogen, the first step of weight loss occurs at approximately $300^{\circ} \mathrm{C}$ and $400^{\circ} \mathrm{C}$ respectively. The second weight loss occurs at approximately at $375^{\circ} \mathrm{C}$ in air and $500^{\circ} \mathrm{C}$ in nitrogen. For Pyrogel materials, in air and nitrogen, the first step of weight loss occurs at approximately $150^{\circ} \mathrm{C}$ and $200^{\circ} \mathrm{C}$ respectively. The second weight loss occurs at approximately at $650^{\circ} \mathrm{C}$ in air and $750^{\circ} \mathrm{C}$ in nitrogen. The first weight loss region corresponds to the removal of residuals PET from the blanket surface and the second weight loss period is associated with the removal of residual fiberglass and opacifiers in the aerogel blanket samples. The remaining material after the TGA was completed with silica gel. Using the weight loss in the temperature range and knowing the temperature of combustion or evaporation of the different ingredient of the investigated aerogel blanket, the percentage of their components can be determined [112].

The thermogravimetric curves for pyrogel blankets differ significantly from those for the spaceloft blankets. The difference in the weight loss profiles between the two groups of aerogel blankets can be attributed to their surface coverage of different composite materials. The surface coverage of PET for Pyrogel blankets whereas the surface coverage of zirconium for Spaceloft blankets. The comparison of the thermogravimetric curves reveals much higher weight loss for Pyrogel blankets corresponding to the phase decomposition of PET for which is not observed for pyrogel blankets . Spaceloft blankets had similar TGA curves around $350-500^{\circ} \mathrm{C}$, suggesting that 
the decomposition of amorphous silica hydroxides followed by the formation of silica oxides. The main weight loss for spaceloft blanket AR 3103X is mainly due to the decomposition and evaporation of coating layer [113]. Moreover, the weight loss for AR3103 was $5 \%$ higher than that of the coated one blanket AR3103X. This might be due to the presence of polymer on the surface of silica oxide nanoparticles. Thicker aerogel blankets have higher values in the thermogravimetric curve pattern is due to the thickness factor. The thickness factor has a main effect on the thermal stability and weight loss of the aerogel blankets because the transfer of the heat produced during the chemical reaction increases the temperature gradient over such small distances. Consequently, smaller and lager particles will have different heating rate.

The TGA investigations in air and nitrogen show different degradation behavior of the flexible aerogel blankets. The onset temperature and the weight percentage of the remaining sample is mainly higher in nitrogen than in air due to the presence of oxygen and the formation of some additional products of the autooxidation in air. With the insertion of oxygen into the macromolecular of the samples, thermal effect will increase. Oxygen thermally induced the scission of the polymer chain inside the sample followed by a process of depolymerisation of the materials. The products of the autooxidation enhance the degradation process. This result can be explained by a clear correlation between the ingredients of the aerogel blanket in air and nitrogen atmosphere and the resistance to higher temperature can be found. Moreover, the degradation in air is followed by the development of some multi mechanisms favoring degradation such as oxidation, thermal and acidolysis [114]. 
The thermal stability and weight loss rates of the blanket materials were also measured with TGA isothermal tests with a constant heating rate. TGA isothermal was used to estimate the thermal decomposition rate of flexible aerogel blankets in the long period of time applications (450 minutes). Figure 4.29 and 4.30 showed the TGA thermograms of the flexible aerogel blankets heated at $375{ }^{\circ} \mathrm{C}$ under air.

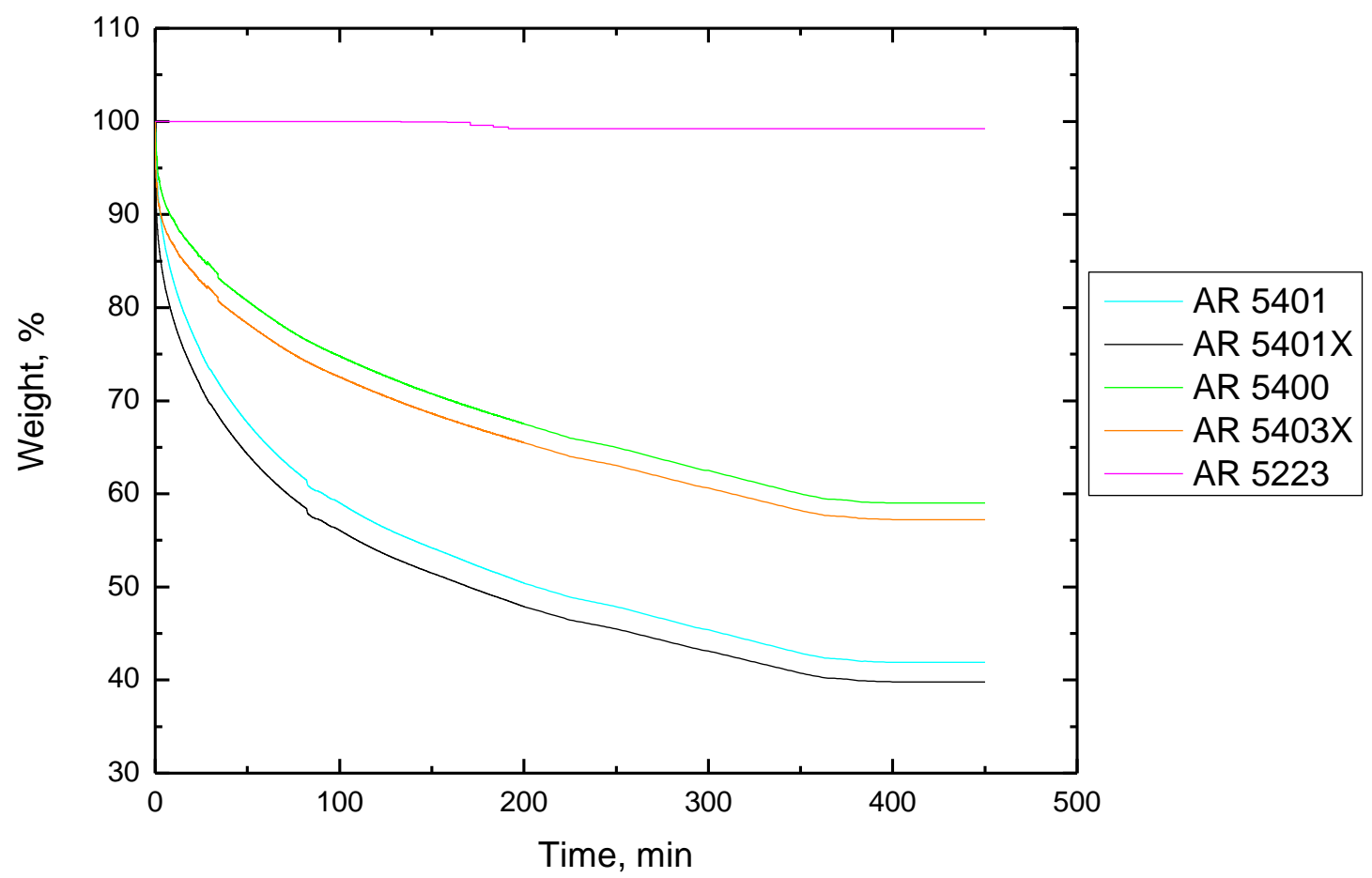

Figure 4.29: Isothermal TGA thermograms of Pyrogel blanket materials in air at $T=375^{\circ} \mathrm{C}$ 


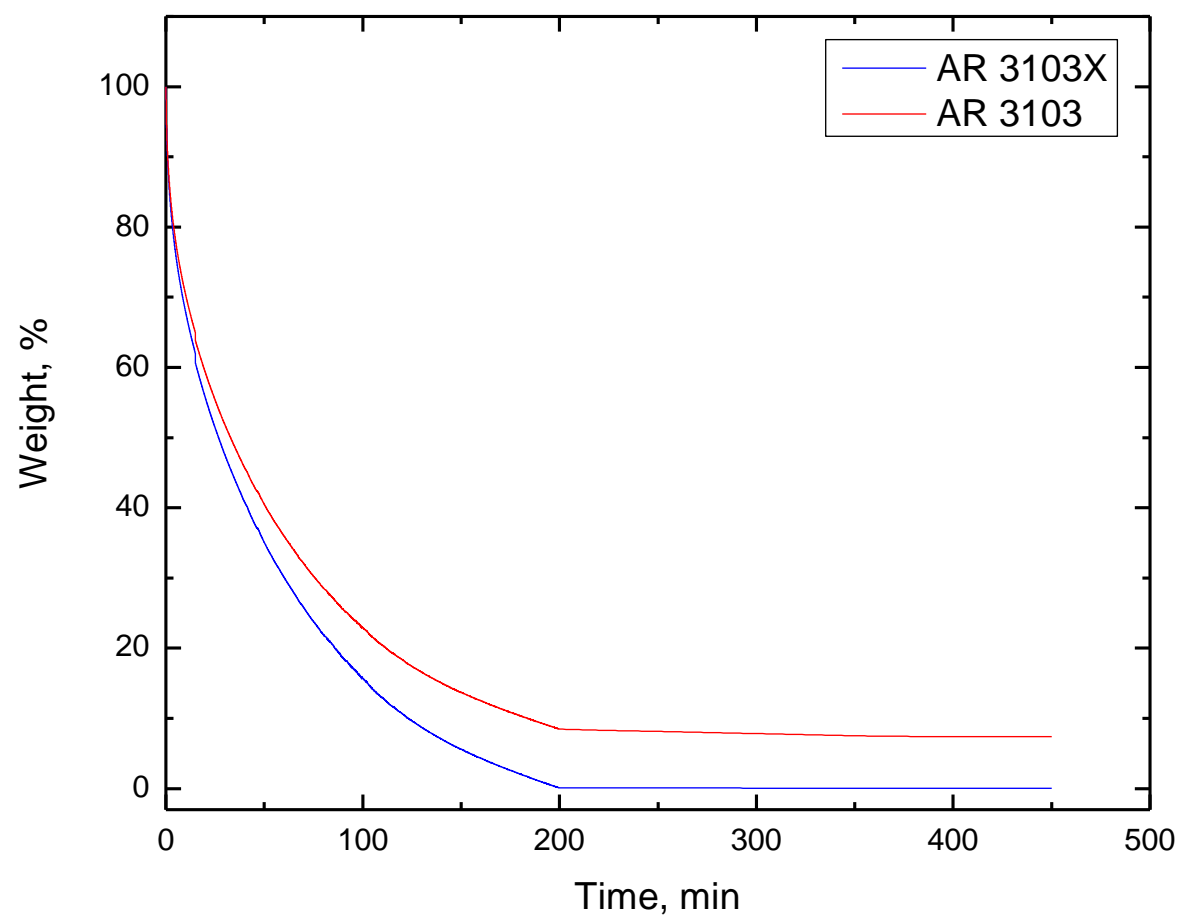

Figure 4.30: Isothermal TGA thermograms of Spaceloft blanket materials in air at $T=375^{\circ} \mathrm{C}$

TGA thermograms of the flexible aerogel blanket materials heated at $375{ }^{\circ} \mathrm{C}$ under nitrogen are shown in figure 4.31 and 4.32 . 


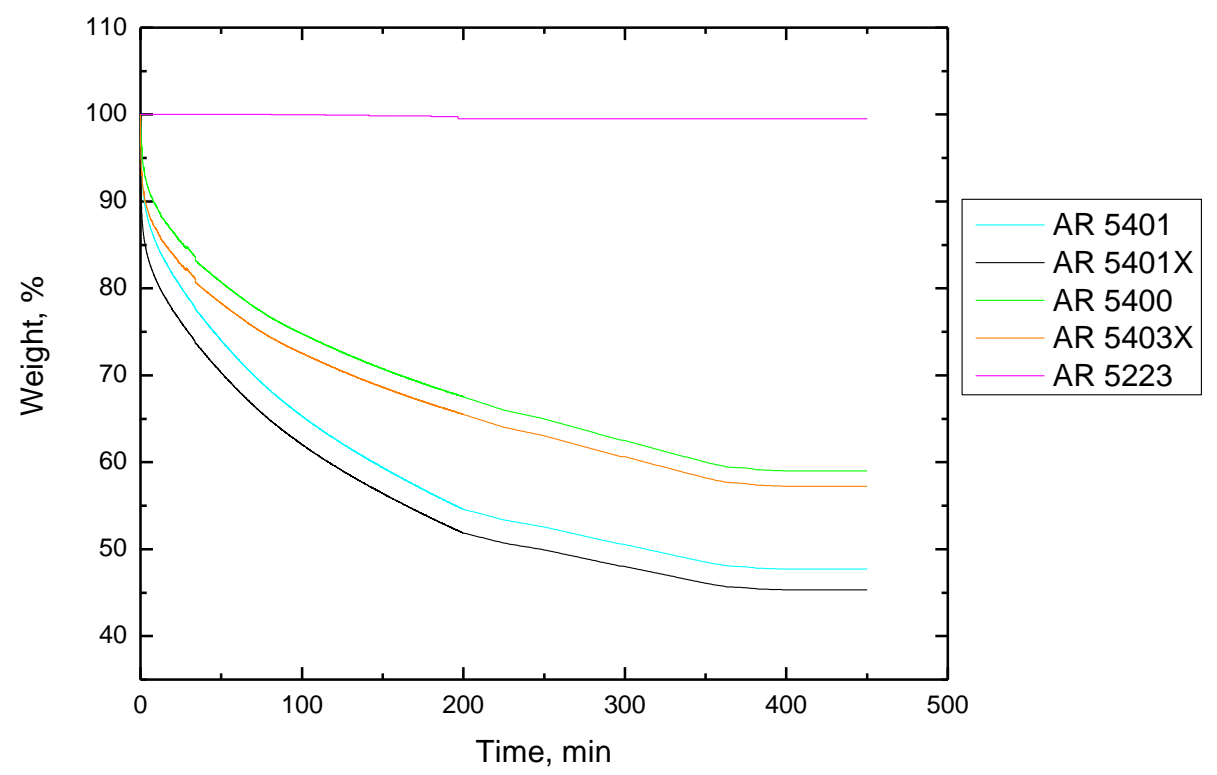

Figure 4.31: Isothermal TGA thermograms of Pyrogel blanket materials in nitrogen at $T=375^{\circ} \mathrm{C}$

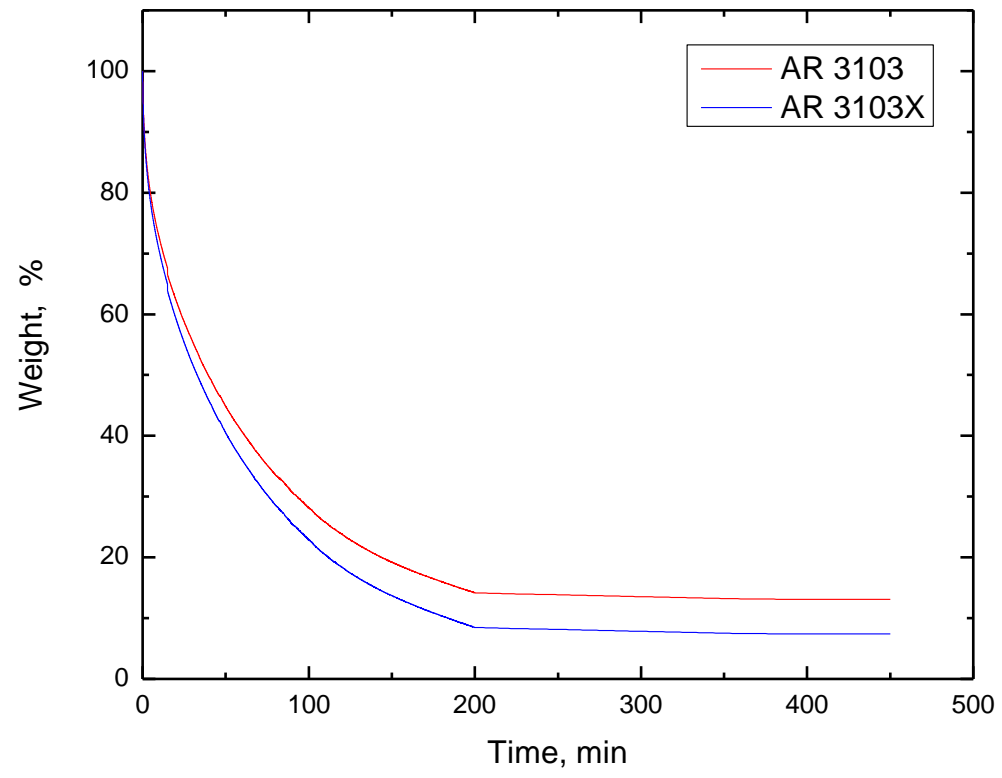

Figure 4.32: Isothermal TGA thermograms of Spaceloft blanket materials in nitrogen at $T=375^{\circ} \mathrm{C}$ 
Pyrogel blankets have higher thermal stability and lower decomposition rates than the Spaceloft blankets in either nitrogen or air. For the same amount loss of weight of materials, it is reached with larger amount of time in nitrogen. Consequently, all the flexible aerogel blankets lost less weigh in nitrogen than in air. The degradation time for coated aerogel blankets are higher than that the ones with naked surface. The results were in good agreement with the results obtained from the heating scanning TGA experiments. This large weight loss of Spaceloft blanket 3 and 4 could be correlated to the poor initial thermal stability of their chemical compound. These TGA curves are made of two major parts. The first region occurs from 0 to 200 minutes and corresponds to the major weight loss. In the second region, 200-450 minutes, the curves leveled off and the weight loss rate is slower.

\subsubsection{Volume percent of fiber and aerogel determination by TGA}

Assuming that there were no voids in the flexible aerogel blanket composites, and they only consists of fibers plus silica aerogel particles, the blanket weight can be determined using the following equation:

$$
W_{f a b}=W_{\text {fiber }}+W_{\text {aerogel }}=f_{\text {fiber }} W_{f a b}+f_{\text {aerogel }} W_{f a b} \quad \text { Equation 4.1 }
$$

The weight percentage of the fiber $f_{\text {fiber }}$ and the silica aerogel particles $f_{\text {aerogel }}$ can be obtained using the thermogram curves in figures 4.34-4.38.

The volume percent of the fiber and the silica aerogel would be:

$$
\begin{array}{ccc}
\% V_{\text {fiber }}=\frac{V_{\text {fiber }}}{V_{\text {fiber }}+V_{\text {aerogel }}}=\frac{W_{\text {fiber }} / \rho_{\text {fiber }}}{\left(W_{\text {fiber }} / \rho_{\text {fiber }}\right)+\left(W_{\text {aerogel }} / \rho_{\text {aerogel }}\right)} & \text { Equation 4.2a } \\
\% V_{\text {fiber }}=\frac{f_{\text {fiber }} / \rho_{\text {fiber }}}{\left(f_{\text {fiber }} / \rho_{\text {fiber }}\right)+\left(f_{\text {aerogel }} / \rho_{\text {aerogel }}\right)} & \text { Equation 4.2b }
\end{array}
$$




$$
\begin{array}{ccc}
\% V_{\text {aerogel }}=\frac{V_{\text {aerogel }}}{V_{\text {fiber }}+V_{\text {aerogel }}}=\frac{W_{\text {aerogel }} / \rho_{\text {aerogel }}}{\left(W_{\text {fiber }} / \rho_{\text {fiber }}\right)+\left(W_{\text {aerogel }} / \rho_{\text {aerogel }}\right)} & \text { Equation 4.3a } \\
\% V_{\text {aerogel }}=\frac{f_{\text {aerogel }} / \rho_{\text {aerogel }}}{\left(f_{\text {fiber }} / \rho_{\text {fiber }}\right)+\left(f_{\text {aerogel }} / \rho_{\text {aerogel }}\right)} & \text { Equation 4.3b }
\end{array}
$$

\subsubsection{Void content, fiber and aerogel volume determination by TGA}

From the electron miscroscopy images of the flexible aerogel blanket, one can see that the blanket composites contain some volume voids. Let the void content be defined as:

Void content $=\frac{V_{v}}{V_{f a b}}=\frac{V_{f a b}-\left(V_{f i b e r}+V_{\text {aerogel }}\right)}{V_{f a b}}$

The theoretical density of the aerogel blanket composite, $\rho_{\text {fab }}^{\circ}$, is defined as the density of the composite in the absence of voids:

$$
\rho_{\text {fab }}^{\circ}=\frac{W_{f a b}}{\left(V_{\text {fiber }}+V_{\text {aerogel }}\right)}=\frac{W_{f a b}}{\left(W_{\text {fiber }} / \rho_{\text {fiber }}\right)+\left(W_{\text {aerogel }} / \rho_{\text {aerogel }}\right)}
$$

Rearinging equation 4.5 and pluging it into equation 4.4 , we have:

$$
\text { Void content }=\frac{\left(W_{f a b} / \rho_{f a b}\right)-\left(W_{f a b} / \rho_{f a b}^{\circ}\right)}{W_{f a b} / \rho_{f a b}^{\circ}}=\frac{\rho_{f a b}-\rho_{f a b}^{\circ}}{\rho_{f a b}^{\circ}}
$$

Equation 4.6

The actual volume percent of the fiber and the silica aerogel would be:

$$
\begin{gathered}
\% V_{\text {fiber }}=\frac{V_{\text {fiber }}}{V_{\text {fiber }}+V_{\text {aerogel }}+V_{v}}=\frac{f_{\text {fiber }}}{\rho_{\text {fibeer }}} \rho_{\text {fab }} \\
\% V_{\text {aerogel }}=\frac{V_{\text {aerogel }}}{V_{\text {fiber }}+V_{\text {aerogel }}+V_{v}}=\frac{f_{\text {aerogel }}}{\rho_{\text {aerogel }}} \rho_{\text {fab }}
\end{gathered}
$$

The density of the Pyrogel and Spaceloft blankets were taken from the ASPEN Aerogels 
product data sheet to be 0.18 and $0.15 \mathrm{~g} / \mathrm{cm}^{3}$ respectively. The density of the fiber and aerogel materials were taken to be 0.24 and $0.09 \mathrm{~g}_{ } \mathrm{cm}^{3}$ respectively. The volume percent of the fiber, aerogel and voids calculated from the weight using equations 4.5-4.8 on flexible aerogel composites is shown in table 4.3 and 4.4.

Table 4.3 Pyrogel blanket components volume fraction determination

\begin{tabular}{|l|c|l|l|l|l|l|}
\hline & $\begin{array}{l}\text { Weight } \\
\text { percentage } \\
\text { of aerogel } \\
\%\end{array}$ & $\begin{array}{l}\text { Weight } \\
\text { percentage } \\
\text { of fiber } \\
\%\end{array}$ & $\begin{array}{l}\text { Theoretical } \\
\text { aerogel } \\
\text { blanket } \\
\text { density } \\
\mathrm{g} / \mathrm{cm}^{3}\end{array}$ & $\begin{array}{l}\text { void } \\
\text { content } \\
\%\end{array}$ & $\begin{array}{l}\text { Actual } \\
\text { aerogel } \\
\text { volume }\end{array}$ & $\begin{array}{l}\text { Actual } \\
\text { fiber } \\
\text { volume }\end{array}$ \\
\hline AR 5223 & 38 & 62 & 0.1469 & 22.5 & 48.08 & 29.42 \\
\hline AR 5400 & 28 & 72 & 0.1636 & 10.0 & 45.81 & 44.18 \\
\hline AR 5403X & 27 & 73 & 0.1655 & 8.75 & 45.31 & 45.94 \\
\hline AR 5401 & 25 & 75 & 0.1684 & 6.25 & 44.11 & 49.63 \\
\hline AR 5401X & 23 & 77 & 0.1735 & 3.75 & 42.67 & 53.57 \\
\hline Average & 28.2 & 71.8 & 0.1638 & 10.25 & 45.20 & 44.55 \\
\hline $\begin{array}{l}\text { Standard } \\
\text { Deviation }\end{array}$ & 5.807 & 5.807 & 0.0101 & 7.256 & 2.015 & 9.20 \\
\hline
\end{tabular}

Table 4.4 Spaceloft blanket components volume fraction determination

\begin{tabular}{|c|c|c|c|c|c|c|}
\hline Sample & $\begin{array}{l}\text { Weight } \\
\text { percentage } \\
\text { of aerogel } \\
\%\end{array}$ & $\begin{array}{l}\text { Weight } \\
\text { Percentage } \\
\text { of fiber } \\
\%\end{array}$ & $\begin{array}{l}\text { Theoretical } \\
\text { aerogel } \\
\text { blanket } \\
\text { density } \\
\mathrm{g} / \mathrm{cm}^{3}\end{array}$ & $\begin{array}{l}\text { void } \\
\text { content } \\
\%\end{array}$ & $\begin{array}{l}\text { actual } \\
\text { aerogel } \\
\text { volume }\end{array}$ & $\begin{array}{l}\text { Actual } \\
\text { fiber } \\
\text { volume }\end{array}$ \\
\hline AR 3103 & 55 & 45 & 0.1252 & 19.79 & 62.19 & 18.83 \\
\hline AR 3103X & 49 & 51 & 0.1321 & 13.54 & 61.38 & 24.27 \\
\hline Average & 52 & 48 & 0.12865 & 16.665 & 61.785 & 21.55 \\
\hline $\begin{array}{l}\text { Standard } \\
\text { Deviation }\end{array}$ & 4.243 & 4.243 & 0.005 & 4.419 & 0.573 & 3.847 \\
\hline
\end{tabular}




\subsection{Contact angle measurement results and discussions}

Figure 4.33 and 4.34 show the microscopy images of the water drop on the Pyrogel and Spaceloft blanket surfaces respectively.

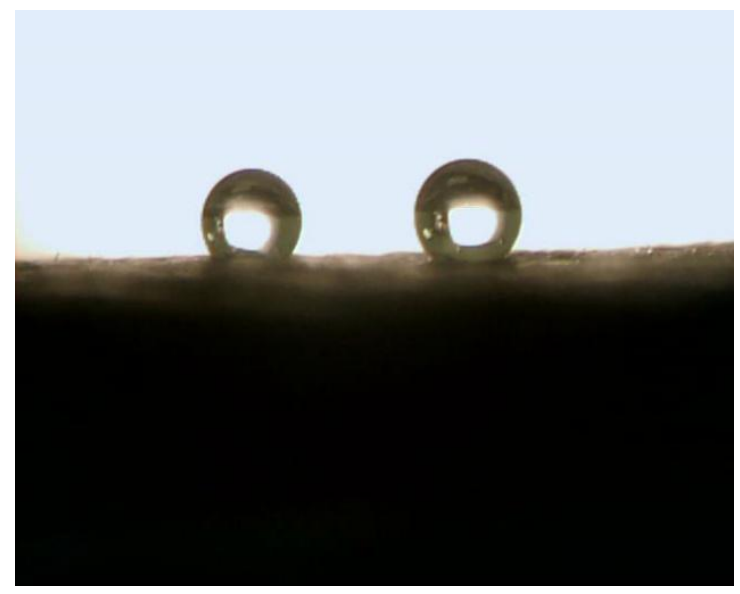

(a)

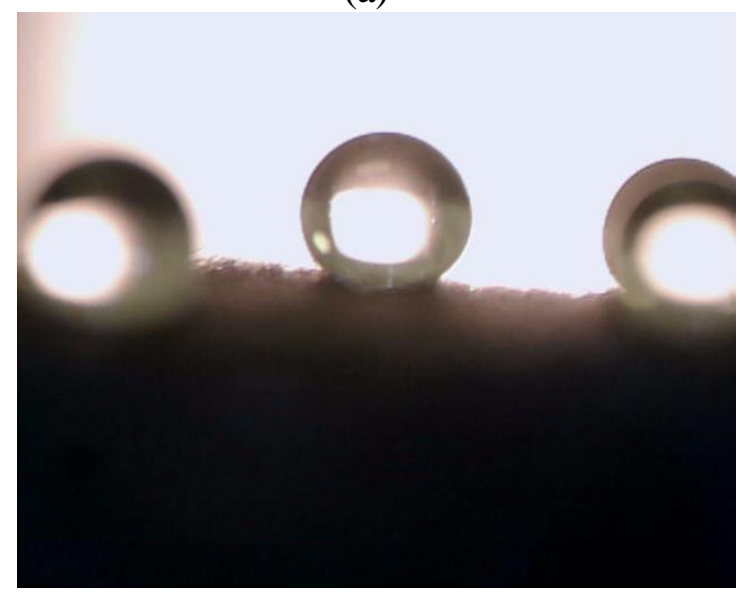

(c)

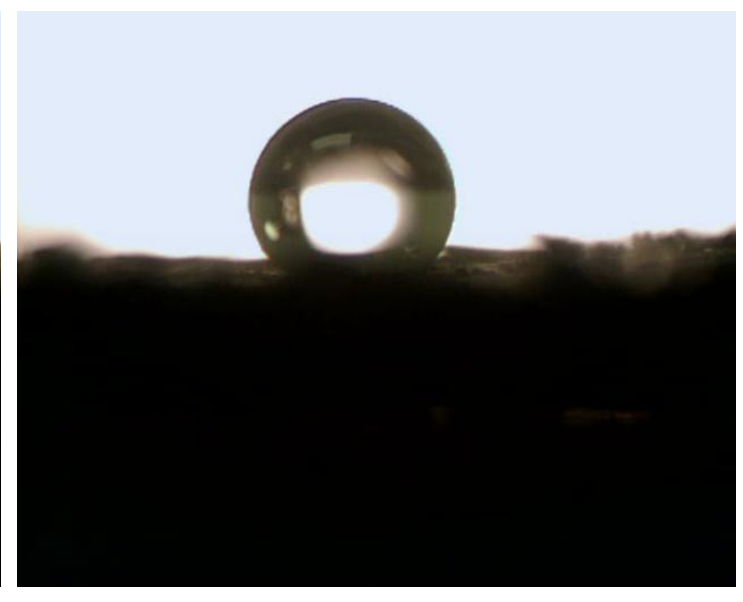

(b)

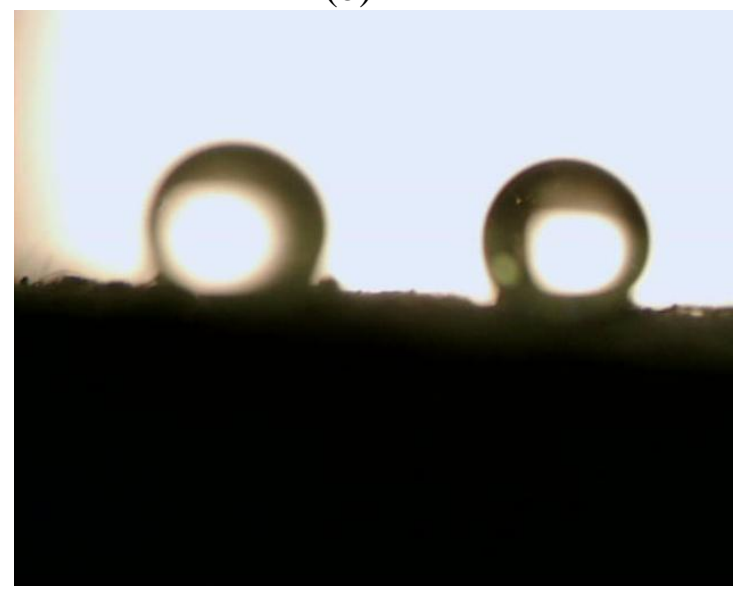

(d) 


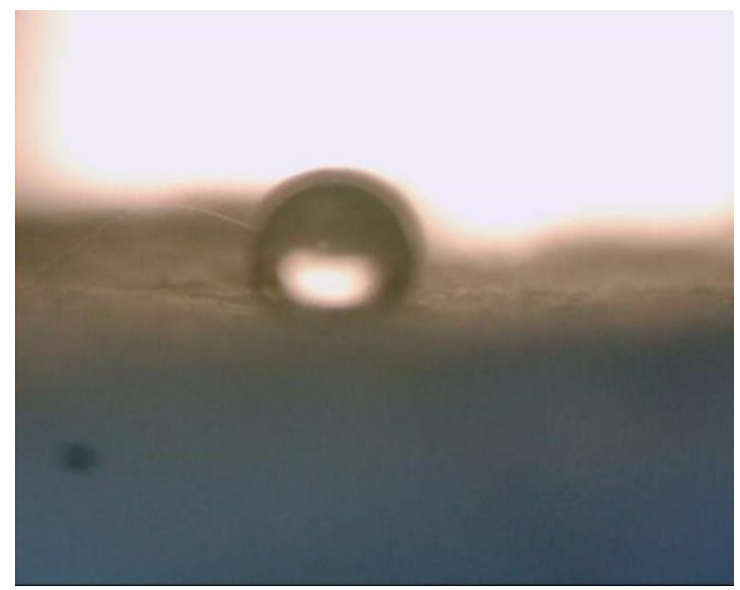

(e)

Figure 4.33: Water drop on Pyrogel blanket surfaces: (a) AR 5401, (b) AR 5401X, (c) AR 5400, (d) AR 5403X, (e) AR 5223

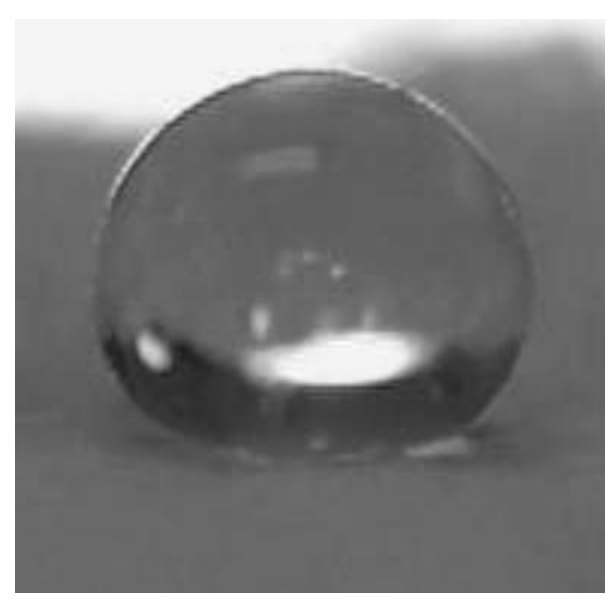

(a)

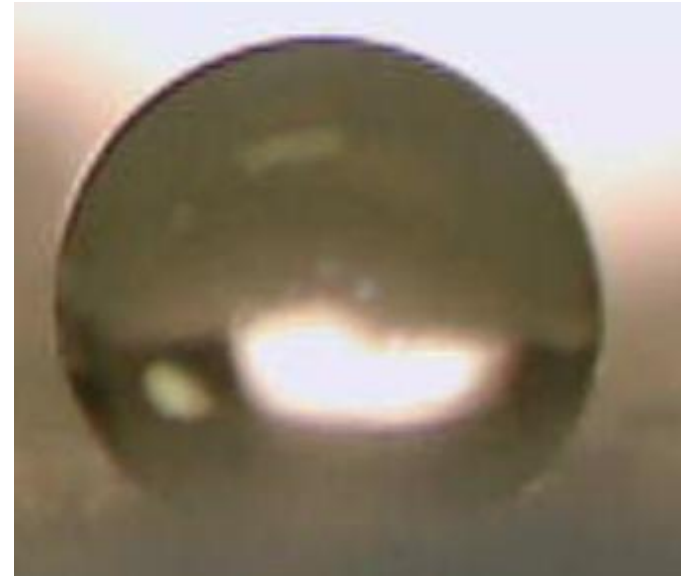

(b)

Figure 4.34: Water drop on Spaceloft blanket surfaces: (a) AR 3103, (b) AR 3103X

Table 4.5 gives the average and standard deviation of contact angles. 
Table 4.5 Contact angle between water drop and aerogel blanket surfaces

\begin{tabular}{|l|l|l|c|}
\hline Sample & $\begin{array}{l}\text { Number of } \\
\text { drops }\end{array}$ & $\begin{array}{l}\text { Contact angle, } \\
\text { Degrees }\end{array}$ & $\begin{array}{c}\text { Standard deviation, } \\
\text { Degrees }\end{array}$ \\
\hline AR 3103 & 4 & 132.423 & \pm 3 \\
\hline AR 3103 X & 4 & 134.103 & \pm 2 \\
\hline AR 5223 & 5 & 134.983 & \pm 3 \\
\hline AR 5403X & 5 & 137.23 & \pm 4 \\
\hline AR 5400 & 5 & 135.166 & \pm 3 \\
\hline AR 5401 & 4 & 134.98 & \pm 2 \\
\hline AR 5401X & 4 & 133.361 & \pm 3 \\
\hline
\end{tabular}

The measurement of the contact angle was used to determine the hydrophobicity of aerogel blankets. In general, it has been considered that the larger the contact angle value, the more the hydrophobicity of the investigated aerogel blanket materials. From the data presented in table 1, it appears that the contact angles are similar for all blankets and their high values indicate that the flexible aerogel blankets investigated are hydrophobic and are unaffected by the moisture. The hydrophobic property of the flexible aerogel blankets is a major requirement for their long term use in the thermal insulating systems. The silica aerogel component of the flexible aerogel blanket is responsible for the hydrophibic nature of the blankets. Although aerogel structures contain the $\mathrm{Si}-\mathrm{OH}$ hydroxyl groups which promote the absorbtion of water, they become hydrophobic after the replacement of $\mathrm{Si}-\mathrm{OH}$ hydroxyl groups by hydrolytically stable $\mathrm{Si}-\mathrm{R}\left(\mathrm{R}=\mathrm{CH}_{3}\right)$ groups thanks to the incorporation of certain hydrophobic reagent such as TMOS in the alcosol itself. Moreover, the surface chemical modification of aerogels using gaseous reagents makes them hydrophobic. 


\section{Chapter 5 Theoretical Models of Effective Thermal Conductivity of Flexible Aerogel Blanket}

\subsection{Background}

Numerous theoretical and numerical approaches have been proposed to solve the problem of predicting the effective thermal conductivity of composite materials. Effective thermal conductivity is defined as a parameter used to measure the heat that flows through a composite material having dimensions much bigger than the scale of its inhomogeneities [115]. Maxwell [116] developed a non-interactive model of spheres dispersed in a continuous matrix by solving Laplace's equation to obtain the effective thermal conductivity of materials containing two components. The model proposed by Russell [117] to calculate the effective thermal conductivity of heterogeneous materials used an Ohm's law method to develop a relationship between the thermal conductivity of composite materials, its component thermal conductivity and their volume fractions. The geometry of Russell's model was made from two cases: one having a continuous split phase with same-sized isolated cubes and the other a continuous porous phase with discontinuous same-sized cubes. A brief description of some theoretical approaches for predicting the effective thermal conductivity of particle filled composite materials were reviewed by Kumlutas et al.[118] who mentioned the Tsao's probalistic models [119], the Cheng and Vachon models [120] and the Lewis and Nielsen semi-theoretical models [121].

Over several years, the prediction of effective thermal conductivity models has been extended to inorganic composites by Progelhof and co-workers [122], to soils by Farouki [123] and to foods by Murakami and Okos [124]. On comparing their 
experimental results, it was found that these models were inaccurate.

The determination of the effective thermal conductivity of heterogeneous materials first requires a microscopic characterization of the materials. A representative microscopic image of the untested flexible aerogel blanket Pyrogel AR 5400, shown in figure 5.1, was introduced to propose predictive models of the effective thermal conductivity of the aerogel composite.

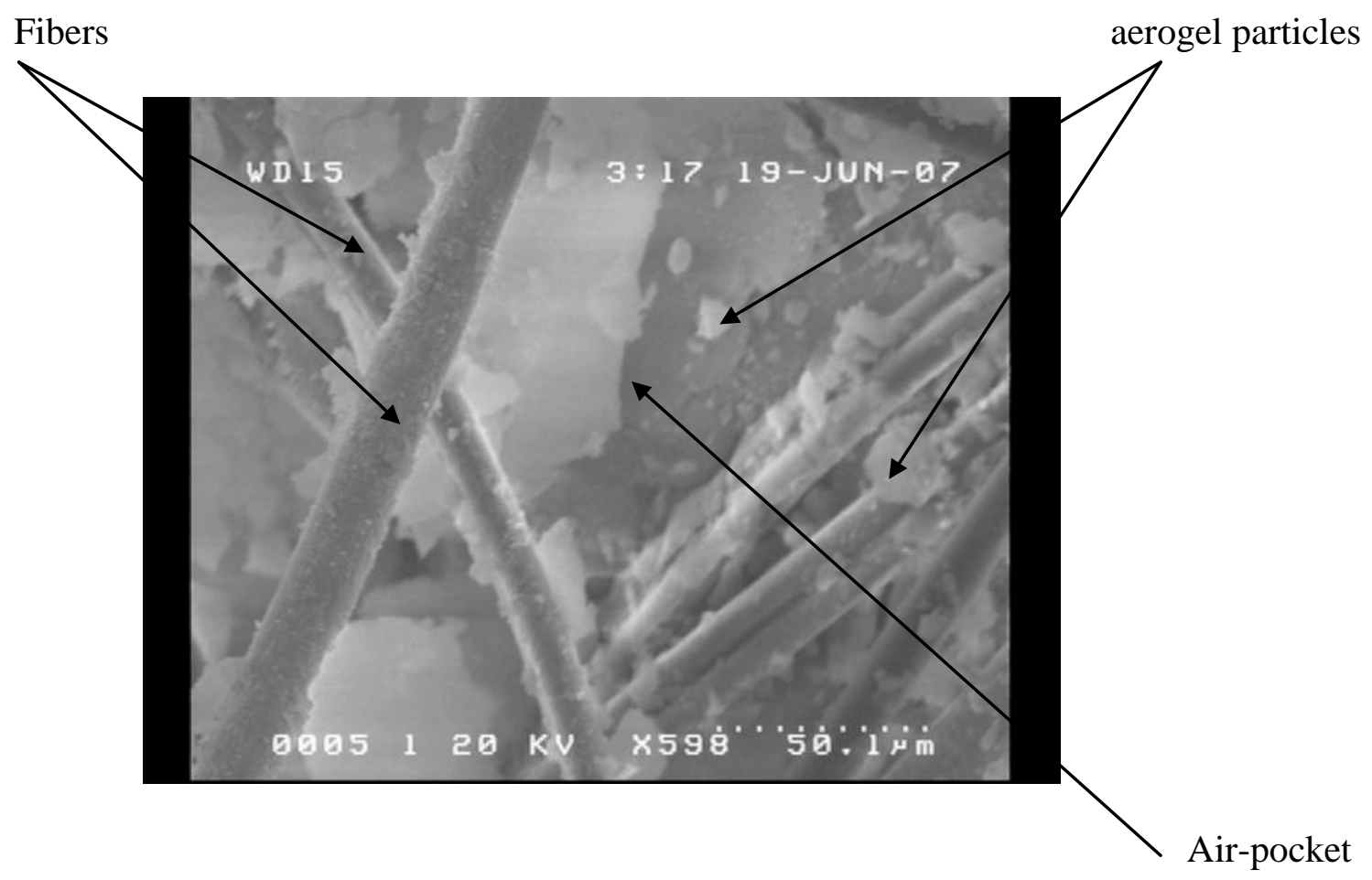

Figure 5.1: SEM image of a flexible aerogel blanket AR 5400

To analyze this SEM image, Nothern Eclipse software was used to count the objects and calculate their size, their area and their percentage of the total area. Nothern Eclipse is a powerful image acquisition, processing, analysis, automation and deconvolution software created and developed by Microscopy Vision and Image Analysis, Inc. (MVIA). Figure 5.2 outlines the fibers, the air-pockets and aerogel 
particles inside the flexible blanket for the image analysis.

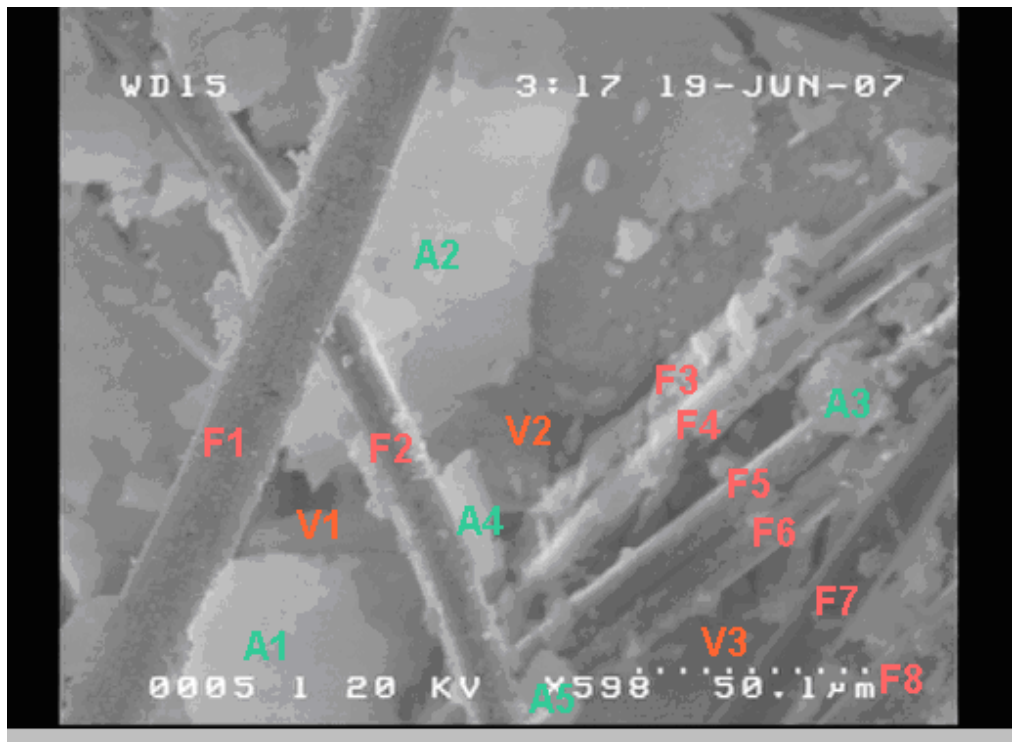

F represents fibers; A represents aerogel particles; V represents air-pockets

Figure 5.2: Quantitative analysis of an SEM image of a flexible aerogel blanket AR 5400

A summary of the particles counted and areas measured is shown in table 5.1

Table 5.1 Data windows of SEM image

\begin{tabular}{|c|c|c|c|}
\hline & \multicolumn{3}{|c|}{ FIBERS } \\
\hline \# Fibers: & 8 & Total Area & 11463.00 \\
\hline Obj\# & Area & $\begin{array}{l}\text { Percent } \\
\text { Area }\end{array}$ & $49.45 \%$ \\
\hline $\mathrm{F} 1$ & 3232.497 & & \\
\hline F2 & 2323.837 & & \\
\hline F3 & 890.6303 & & \\
\hline F4 & 1440.798 & & \\
\hline F5 & 1224.64 & & \\
\hline F6 & 958.7608 & & \\
\hline F7 & 915.1118 & & \\
\hline F8 & 476.7235 & & \\
\hline TOTAL: & 11463.00 & & \\
\hline
\end{tabular}

\begin{tabular}{|c|r|l|r|}
\hline & & $\begin{array}{l}\text { AlR } \\
\text { POCKETS }\end{array}$ & \\
\hline $\begin{array}{l}\text { \# Air- } \\
\text { pockets: }\end{array}$ & 6 & Total Area & 2600.09 \\
\hline Obj\# & Area & $\begin{array}{l}\text { Percent } \\
\text { Area }\end{array}$ & $11.21 \%$ \\
\hline V1 & 987.7969 & & \\
\hline V2 & 718.8808 & & \\
\hline V3 & 614.3129 & & \\
\hline$\ldots$ & & & \\
$\ldots$. & 279.0994 & & \\
$\ldots .$. & & & \\
$\ldots .$. & & & \\
\hline TOTAL: & 2600.09 & & \\
\hline
\end{tabular}




\begin{tabular}{|c|r|l|l|}
\hline & \multicolumn{3}{|c|}{ AEROGELS } \\
\hline \# Aerogels: & 20 & Total Area & 9118.66 \\
\hline Obj\# & Area & $\begin{array}{c}\text { Percent } \\
\text { Area }\end{array}$ & $39.33 \%$ \\
\hline A1 & 1628.299 & & \\
\hline A2 & 4260.716 & & \\
\hline A3 & 316.7403 & & \\
\hline A4 & 178.5815 & & \\
\hline A5 & 149.5454 & & \\
\hline$\ldots$. & 2584.778 & & \\
$\ldots .$. & & & \\
\hline TOTAL: & 9118.66 & & \\
\hline
\end{tabular}

All sizes are in micrometers.

The quantitative phase analysis results of the SEM image are used to provide input for the theoretical and numerical modeling. As shown in figure 5.1, the microstructure of the flexible aerogel blankets consists of polyethylene (PET) fibers, aerogel particles and air-pockets. Therefore, predicting the effective thermal conductivity of this composite material requires not only the determination of the values of the thermal conductivities of each constituent but also the volume fractions, shapes, sizes and orientations. The volume fraction of the flexible aerogel blanket is given by:

$$
V_{f a b}=V_{\text {fiber }}+V_{\text {aerogel }}+V_{\text {air }}=1 \quad \text { Equation 5.1 }
$$

There are inherent difficulties in the theoretical prediction of the effective thermal conductivity of composite materials. Several simplifications have to be made to reach simple and accurate theoretical prediction models. 


\subsection{Assumptions}

Theoretical models, used for predicting thermal conductivity of composite materials may be distinguished by the nature of the assumptions. The following common assumptions are shared by almost all the theoretical approaches [125]:

- Macroscopic homogeneity of the flexible aerogel blankets

- The fibers, the aerogel particles and the air-pockets are locally homogeneous and isotropic

- The fibers, the aerogel particles and the air-pockets are in direct contact and bonded so that slip can’t occur at a phase interface

- Completely absence of thermal contact resistance between the phase surfaces

- There are no cracks in the fibers

- The overall average thermal conductivity of the material will be the main parameter rather than localized variations of the thermal conductivity in the material.

\subsection{Thermal Conductivity of the Constituents of the Aerogel Composites}

The basic law of heat conduction using indicial notation is:

$$
q_{i}=-k_{i j} \frac{d T}{d x_{j}}
$$

In this equation, thermal conductivity is a second-rank tensor which is not symmetric in the most general case except in the most crystal systems. For isotropic materials, it is likely that $k_{i j}$ can be considered as a scalar number $\mathrm{k}$ [126]. 
The thermal conductivity of the fibers and the aerogel particles were evaluated as function of the heat flow direction. When these materials are oriented in the direction perpendicular to the heat flow, their thermal conductivity is:

$$
\begin{aligned}
& k_{\text {fiber } \perp}=\frac{V_{\text {fiber }}}{k_{\text {fiber }}} \\
& k_{\text {aerogel } \perp}=\frac{V_{\text {aerogel }}}{k_{\text {aerogel }}}
\end{aligned}
$$

Equations 5.3

In the axial direction, the thermal conductivity of the fibers and the aerogel particles can be written as the product of the thermal conductivity of a single particle times the volume fraction of the constituent material:

$$
\begin{gathered}
k_{\text {fiber // }}=k_{\text {fiber }} V_{\text {fiber }} \\
k_{\text {aerogel // }}=k_{\text {aerogel }} V_{\text {aerogel }}
\end{gathered}
$$

For air particles, the thermal conductivity will be related to its volume fraction and to the direction of the heat flow. Therefore, the thermal conductivity of air particles will be computed by:

$$
\begin{array}{ll}
k_{\text {air } \perp}=\frac{V_{\text {air }}}{k_{\text {air }}} & \text { in the transverse direction } \\
k_{\text {air // }}=k_{\text {air }} V_{\text {air }} & \text { in the axial direction }
\end{array}
$$

The thermal properties of the constituent materials $\left(T=200{ }^{\circ} \mathrm{C}\right)$ are given in table 5.2. 
Table 5.2 Values of properties of the constituent materials [127]

\begin{tabular}{|l|l|l|l|l|}
\hline & & & & \\
Constituent & Material & $\begin{array}{l}\text { Thermal } \\
\text { conductivity } \\
\text { W/m-K }\end{array}$ & $\begin{array}{l}\text { Density } \\
\mathrm{kg} / \mathrm{m}^{3}\end{array}$ & $\begin{array}{l}\text { specific } \\
\text { heat } \\
\text { capacity } \\
\mathrm{kJ} / \mathrm{kg} \cdot \mathrm{K}\end{array}$ \\
\hline Fiber & PET & 0.24 & ------ & ----- \\
\hline $\begin{array}{l}\text { main } \\
\text { particles }\end{array}$ & aerogel & 0.003 & 0.18 & 0.703 \\
\hline $\begin{array}{l}\text { Secondary } \\
\text { particles }\end{array}$ & air & 0.0386 & 0.746 & 1.026 \\
\hline
\end{tabular}

\subsection{Thermal Conductivity Models}

To define the range of the effective thermal conductivity predictions of the flexible aerogel blanket, parallel and series structures of its constituent's thermal conductivity with respect to heat flow were used. Figure 5.1(a) and 5.1(b) show a lower and upper bond of the thermal conductivity which are given when materials are arranged in parallel and in series respectively.

\begin{tabular}{|l|l|}
\multicolumn{1}{c|}{} & FIBERS \\
Heat Flow & AEROGEL PARTICLES \\
\cline { 2 - 2 } & AIR-POCKETS \\
\hline
\end{tabular}

(a) 


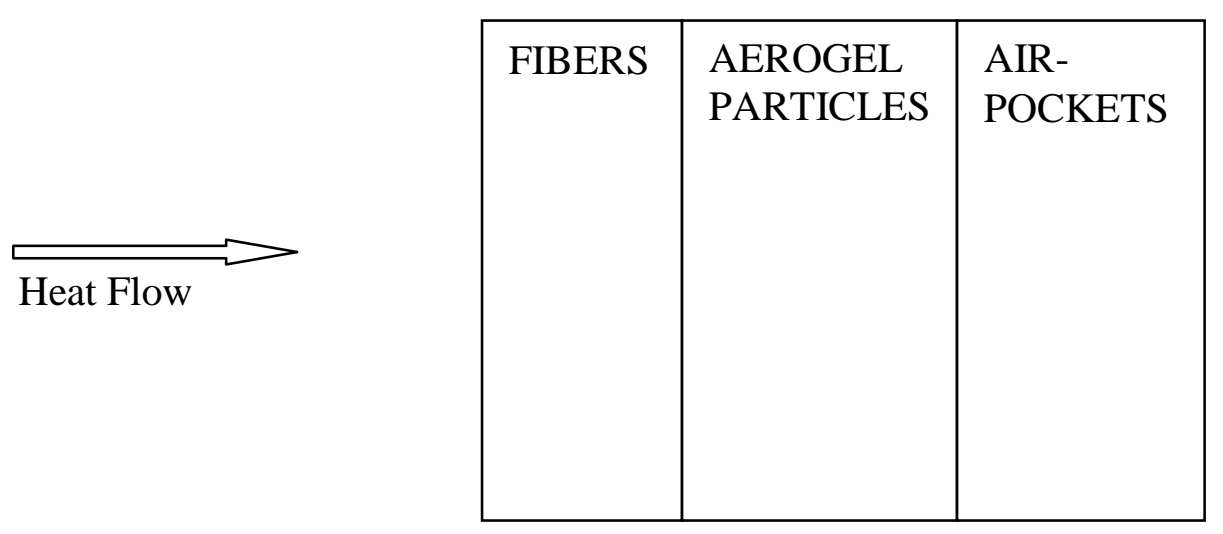

(b)

Figure 5.3: Flexible aerogel blanket (a) parallel and (b) series structures

When the flexible aerogel blanket is thermally in parallel in the direction of heat flow, the rule of mixtures holds for the thermal conductivity as:

$$
\begin{array}{ll}
k_{f a b}=\sum_{i=1}^{3} k_{i} V_{i} & \text { Equation 5.6a } \\
k_{f a b}=k_{\text {fiber }} V_{\text {fiber }}+k_{\text {aerogel }} V_{\text {aerogel }}+V_{\text {air }} k_{\text {air }} & \text { Equation 5.6b } \\
k_{\text {fab }}=\left(k_{\text {fiber } / /}+k_{\text {air }}-k_{\text {air }} V_{\text {fiber }}\right)-V_{\text {aerogel }}\left(-k_{\text {aerogel }}+k_{\text {air }}\right) & \text { Equation 5.6c }
\end{array}
$$

Likewise, when the aerogel composites are thermally in series in the direction of heat flow, their effective thermal conductivity is given by:

$$
k_{f a b}=\left[\sum_{i=1}^{3} \frac{V_{i}}{k_{i}}\right]^{-1}
$$


$k_{\text {fab }}=\left[\left(k_{\text {fiber } \perp}+\frac{1-V_{\text {fiber }}}{k_{\text {air }}}\right)+V_{\text {aerogel }}\left(\frac{k_{\text {air }}-k_{\text {aerogel }}}{k_{\text {air }} \cdot k_{\text {aerogel }}}\right)\right]^{-1}$

Equation 5.7b

The geometric mean model of effective thermal conductivity of the aerogel blanket is obtained from:

$$
\begin{aligned}
& k_{f a b}=\prod_{i=1}^{3} k_{i}^{V_{i}} \\
& k_{f a b}=k_{\text {fiber }}^{V_{\text {fiber }}} \cdot k_{\text {aerogel }}^{V_{\text {aerogel }}} \cdot k_{\text {air }}^{1-V_{\text {fiber }}-V_{\text {aerogel }}} \\
& k_{\text {fab }}=k_{\text {air }}\left(\frac{k_{\text {fiber }}}{k_{\text {air }}}\right)^{V_{\text {fiber }}}\left(\frac{k_{\text {aerogel }}}{k_{\text {air }}}\right)^{V_{\text {aerogel }}}
\end{aligned}
$$

Equation $5.8 \mathrm{~b}$

Equation 5.8c

An attempt has been made to extend Agari and Uno's model [128] for filled

polymers with the aerogel blanket system. With this view, the effective thermal conductivity of the flexible is given by:

$\log k_{f a b}=\sum_{i=1}^{3} C_{i} V_{i} \log k_{i}$ Equation 5.9a

$\log k_{\text {fab }}=C_{\text {fiber }} V_{\text {fiber }} \log k_{\text {fiber }}+C_{\text {aerogel }} V_{\text {aerogel }} \log k_{\text {aerogel }}+$ $C_{\text {air }}\left(1-V_{\text {fiber }}-V_{\text {aerogel }}\right) \log k_{\text {air }}$ Equation 5.9b

$\log k_{\text {fab }}=\left(C_{\text {fiber }} \log k_{\text {fiber }}-C_{\text {air }} \log k_{\text {air }}\right) V_{\text {fiber }}+$ $\left(C_{\text {aerogel }} \log k_{\text {aerogel }}-C_{\text {air }} \log k_{\text {air }}\right) V_{\text {aerogel }}+C_{\text {air }} \log k_{\text {air }}$ Equation 5.9c

In equation 5.9, $C_{\text {fiber }}, C_{\text {aerogel }}$ and $C_{\text {air }}$ are measuring the effect of particles on the 
secondary structure of the composite and the ease to the particles to form conductive chains. They are experimentally determined and their values are closer to 1 [128].

Defined as a weighted harmonic combination of the series and parallel models, the Krischer's model [129] offers a very good contribution to the prediction of the effective thermal conductivity of the flexible aerogel blanket. This model yields the following formulation

$k_{f a b}=\left\lceil Z\left(\sum_{i=1}^{3} \frac{V_{i}}{k_{i}}\right)+\frac{1-Z}{\sum_{i=1}^{3} k_{i} V_{i}}\right\rfloor^{-1}$ Equation 5.10a

Substituting the parallel and series models components by their final expression derived in equations 5.6 and 5.7, the Krischer's model will be reduce to

$k_{f a b}=\left[Z \aleph_{f a b(\text { series } \bmod e l)}+\frac{1-Z}{k_{f a b(\text { parallel } \bmod e l)}}\right]^{-1}$

Equation 5.10b

The weighting parameter $\mathrm{Z}$, also called the distribution factor, can take any values from 0 to 1 . The highest and the lowest values of the range must be excluded to avoid the matching results between two alternative models. In one side, the Krischer model and the series model when $Z=1$, in another side, the Krischer model and the parallel model when $\mathrm{Z}=0$.

The effective medium theory (EMT) [130] can also be used to calculate the effective thermal conductivity of the aerogel composite materials. A general EMT model is formulated as: 


$$
\sum_{i=1}^{3} V_{i} \frac{k_{i}-k_{f a b}}{k_{i}+2 k_{f a b}}=0
$$

The derivation of equation 5.19 was based on the assumption that the temperature gradient within the material is uniform due to the complete random distribution of the embedded components. For materials which don't fulfill the above assumption, the EMT model was modified by the introduction of the so-called "flexible" factor $\phi$ into equation 5.19, which yields the following formulation:

$\sum_{i=1}^{3} V_{i} \frac{k_{i}-k_{f a b}}{k_{i}+(0.5 \phi-1) k_{f a b}}=0$

The dependency of the effective thermal conductivity to the microstructure of a particular composite material is expressed through the "flexible" factor $\varphi$. The determination of factor $\phi$ for a specific composite material requires some numerical modeling or some physical experiments. By simplification, the values of this factor will be selected in the present study.

To express explicitly $k_{\text {fab }}$ from equation 5.12, two steps were used.

The first step was the computation of $k_{e 1}$, the effective thermal conductivity of two components of the flexible aerogel blankets which are the aerogel particles and the fibers. $k_{e 1}$ is obtained from:

$$
k_{e 1}=\frac{1}{\phi-2}\left[\begin{array}{l}
{\left[\left(0.5 \phi \frac{V_{\text {aerogel }}}{1-V_{\text {air }}}-1\right) k_{\text {aerogel }}+\left(0.5 \phi \frac{V_{\text {fber }}}{1-V_{\text {air }}}-1\right) k_{\text {fber }}\right.} \\
\left.++\sqrt{\left[\left(0.5 \phi \frac{V_{\text {aerogel }}}{1-V_{\text {air }}}-1\right) k_{\text {aerogel }}+\left(0.5 \phi \frac{V_{\text {fiber }}}{1-V_{\text {air }}}-1\right) k_{\text {fiber }}\right]^{2}+(2 \phi-4) k_{\text {aerogel }} k_{\text {fber }}}\right]
\end{array}\right.
$$


Then, the EMT model was extended to air-pockets, the third component of the blanket.

The effective thermal conductivity for these three components is calculated by:

$$
k_{f a b}=\frac{1}{\phi-2}\left[\begin{array}{l}
\left(0.5 \phi V_{a i r}-1\right) k_{a i r}+\left(0.5 \phi\left(V_{\text {aerogel }}+V_{f i b e r}\right)-1\right) k_{e 1} \\
+\sqrt{\left(0.5 \phi V_{a i r}-1\right) k_{a i r}+\left(0.5 \phi\left(V_{\text {aerogel }}+V_{\text {fiber }}\right)-1\right) k_{e 1} 2^{2}+(2 \phi-4) k_{a i r} k_{e 1}}
\end{array}\right]
$$

Equation 5.14

\subsection{Results and Discussions}

The results of the variation of the thermal conductivity of the flexible aerogel blanket with the volume fraction of aerogel particles for the models described above are plotted in figure 5.4

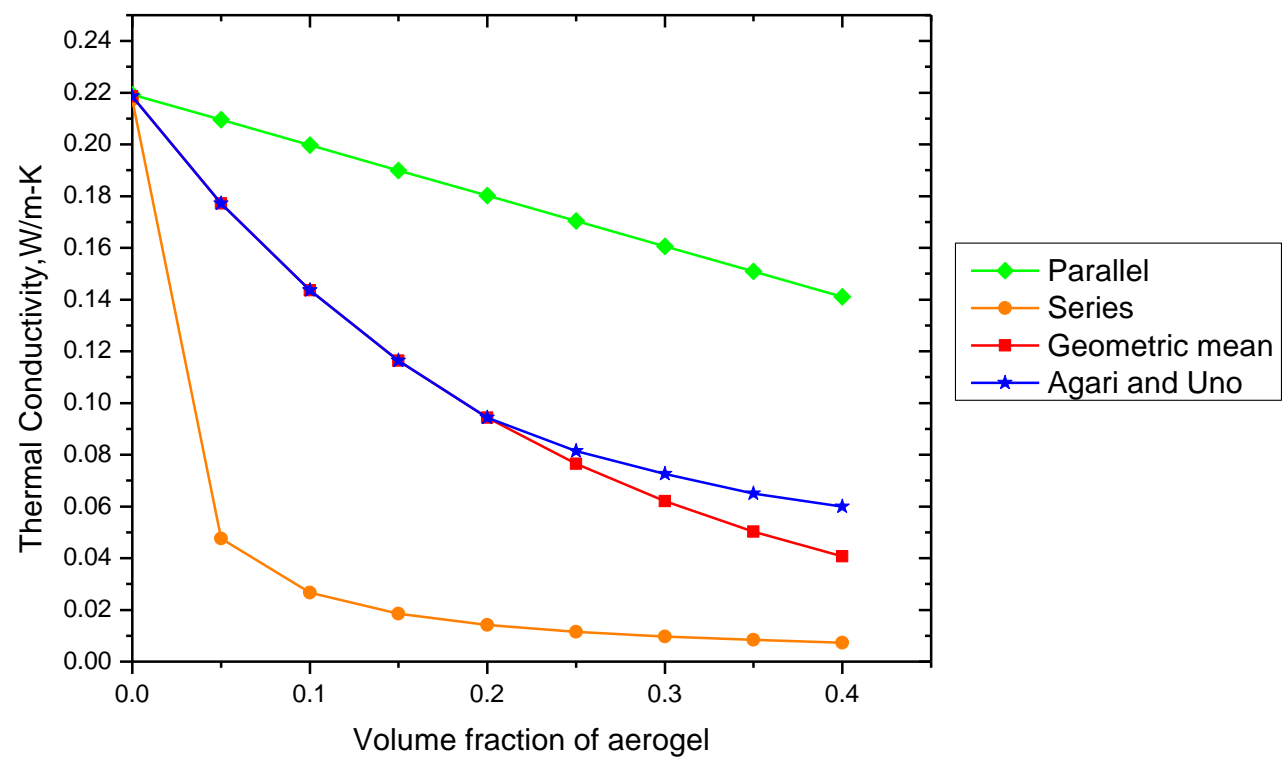

Figure 5.4: Predicted thermal conductivity values for flexible aerogel blankets 
The effect of flexible aerogel blanket orientation with respect to the heat flow vector is apparent by comparing the multiple curves in figure 5.4. It can be seen from this figure that the curves of the effective thermal conductivity of flexible aerogel blankets with respect to the aerogel volume fraction are obtained from the parallel, the series, the geometric mean and the Agari and Uno models. For all of these models, the effective thermal conductivity of the aerogel composite decreased, as the aerogel volume fraction increased. The series model assumed that heat transfer was perpendicular to alternate layers of the aerogel composite, whereas the parallel model assumed that the components of the insulation blankets were parallel to the heat transfer. In the parallel model, the aerogel blankets aligned axially to the heat flow provided a relatively high conductivity pathway across the blanket's components that were less conductive, thereby compromising its overall insulating capacity. When the flexible aerogel blankets were normal to the heat flow as defined by the series model, they provided no such direct conduction pathway and required heat transfer to occur primarily through the blanket's components. Consequently, the series model gave the lowest value for the effective thermal conductivity of the flexible aerogel blankets whereas the parallel model gave the highest value. Models based on the perpendicular heat flows produced more accurate results than the parallel model because the heat flowed through the composite's parts one after another. This provided only one path to the heat flow and allowed the majority of heat transfer to occur through the composite components [73].

The geometric mean and the Agari and Uno models predicted values in between the parallel and series models. Agari and Uno introduced coefficients $C_{i}$ to the geometry 
mean model in order to obtain results more accurate than the geometric mean model.

These coefficients were used to adjust the model to match the experimental and/or the numerical data. The geometric mean and the Agari and Uno models provided significantly better predictions of the thermal conductivities of the flexible aerogel blanket throughout the whole range. There was a reasonable agreement between the geometric and Agari and Uno models

In the Krischer model, heat transfer is assumed to take place by a combination of parallel and perpendicular heat flow. Figure 5.5 shows the variation of the effective thermal conductivity with the volume fraction of aerogel for different values of the distributor factor.

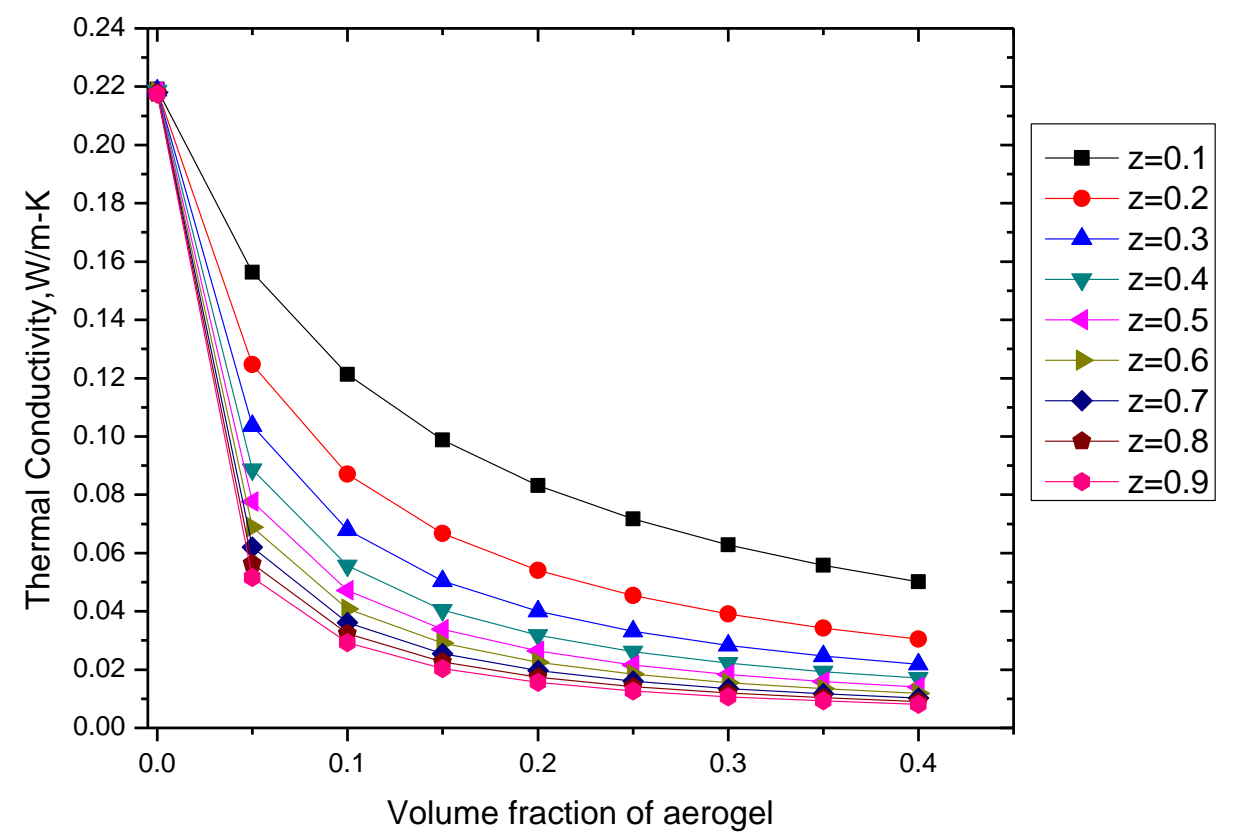

Figure 5.5: Krischer model predicted thermal conductivity values for flexible aerogel blankets

It is noted that the values of thermal conductivities of the aerogel composites for a defined value of the aerogel volume fraction increased gradually with increasing value of 
the distributor factor. The values of the distribution factor are related to the contribution of series and parallel arrangement to the heat transfer mechanism in the actual spatial distribution. In fact, the distribution factor represents the spatial distribution of the components of the flexible aerogel blanket.

The distribution factor was calculated by Murakami and Okos [124] using a third degree polynomial in terms of the volume fraction $\mathrm{V}$ as follow:

$$
Z=A_{1}+A_{2}(V-0.4)+A_{3}(V-0.4)^{2}+A_{4}(V-0.4)^{3}
$$

Where the coefficients $A_{i}$ are defined as:

$$
\begin{aligned}
& A_{i}=a+b \xi+c \xi 2+d \xi 3 \\
& \xi=X \quad \text { for } \mathrm{i}=1,2 \\
& \text { Where } \quad \xi=(X-0.25) \quad \text { for } \mathrm{i}=3 \\
& \xi=(X-0.1627) \quad \text { for } \mathrm{i}=4
\end{aligned}
$$

The values of $\mathrm{a}, \mathrm{b}, \mathrm{c}$ and $\mathrm{d}$ depend on the particular $A_{i}$ and are tabulated.

Figure 5.6 plots the calculated effective thermal conductivity as a function of the volume fraction of aerogel and the flexible factor according to the EMT model. Two values for the flexible factors $\phi$ were selected: $\phi=4$ and $\phi=6$. For $\phi=6$, the original EMT model expressed in equation 5.18 is obtained from equation 5.19. Similar to the trend observed from the results of previous models, the effective thermal conductivity of the flexible aerogel blanket decreased along with aerogel volume fraction. $\phi=4$ was selected to make the EMT model more accurate within the whole composition range of the volume fraction of aerogel. 


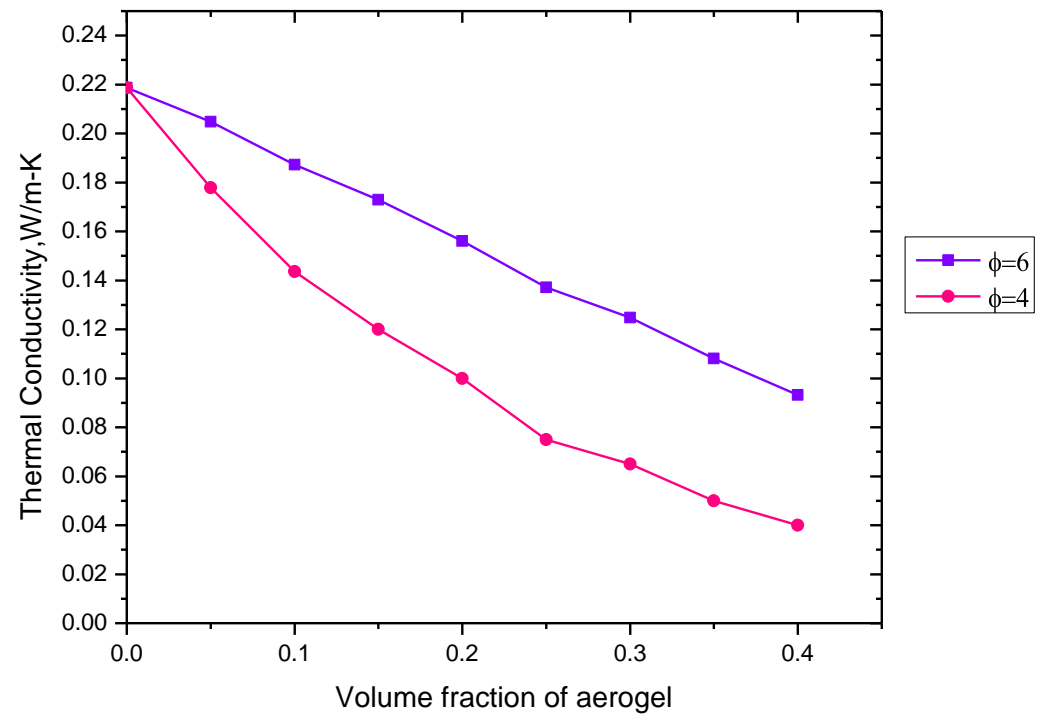

Figure 5.6: EMT model predicted thermal conductivity values for flexible aerogel blankets

Figure 5.6 shows the deviation between the EMT curves with the flexible factor $\phi=6$ and the EMT curve with $\phi=4$ reached its highest value for volume fraction of aerogel $V_{\text {aerogel }}=0.25$. In general, the EMT model gave more accurate effective thermal conductivity results for composite systems in which there was an internal network inside their structure. This model assumed a completely random dispersion of the components inside the material and an average value for the effect of the local distortions to the temperature distribution caused by individual inclusions [130]. 


\section{Chapter 6 Finite Element Analysis (FEA)}

\subsection{Introduction to ANSYS}

Finite Element Methods (FEM) are widely used in many fields of engineering analysis such as stress, deformation, heat transfer, fluid flow, electromagnetic, etc. using commercial as well as special purpose computer codes. Commercial finite element analysis software package ANSYS version 11.0, one of the most versatile, was used for the simulation of thermal property of flexible aerogel blankets. ANSYS software suite has been developed over 30 years by ANSYS Inc. to deal with the simulation needs of all industries. It is a comprehensive advanced software package which contains a suite of powerful engineering simulation programmes, based on the FEM, which can solve a broad range of problems from simple analysis to the most complex non linear simulations. A complete ANSYS analysis usually consists of three distinct stages [131]:

Preprocessing, Solving and Postprocessing.

During the Preprocessing phase, the FEM model is created by selecting the following items:

- The type of element used in assembling the model

- Element geometric property

- Element material properties

- The nodes, their locations, their connections with elements and their boundary conditions

- The loadings, their magnitudes, locations and directions. 
The Solving step is performed using the systems of equations are solved to give the unknowns values at the nodes. These systems of equations can be a set of linear or non-linear equations. The Postprocessing stage display and evaluate the results of the FEA.

\subsection{Finite Element Model}

The effective thermal conductivity of particle and fiber reinforced composites were also predicted using a 3D numerical finite difference and a finite element method. Jones and Pascal [132] computed the thermal conductivity of multi-component materials using a 3-D numerical finite difference scheme. This model analyzed the effect of the quantity and the distribution of each component on the effective thermal conductivity of the material. The applicability of the FEM in predicting the effective transverse thermal conductivity of fiber reinforced composites was investigated by Islam and Pramila[125]. Their FE solutions for composite materials with cracks and composites materials with interfacial thermal barrier resistance were compared to the results of the effective thermal conductivity from experimental and analytical studies. They concluded that the periodicity realized by prescribed temperatures provides the most accurate results with up to $50 \%$ of fiber volume fraction. Floury and co-workers [115] developed a 3-D finite element simulation using the COSMOL Multiphysics software package coupled with MATLAB to calculate the effective thermal conductivity of theoretical composite materials having a dispersed phase of rods or cubes within a continuous matrix. They found that their effective thermal conductivity model provides more accurate values for low conductivity inclusions, with errors ranging from $-3.8 \%$ to $7.6 \%$ than the one for 
high conductivity inclusions. This result is explained by the increase of effective thermal conductivity due to the second phase random form heat bridges which occurs for higher conductivity inclusions.

In this study, a FEM model was proposed to predict the effective thermal conductivity of the flexible aerogel blankets. This method is based on 3-D models used to simulate the microstructure of the insulation blanket for various volume fraction of aerogel. The 3-D models consist of spheres in a rectangular paraleppiped lattice array as shown in figure 6.1. Figure 6.1 depicts the structure of an aerogel blanket made of aerogel particles, air pockets and fiber. The aerogel particles and the air pockets are modeled as spherical while the fiber is modeled as a parallepiped.

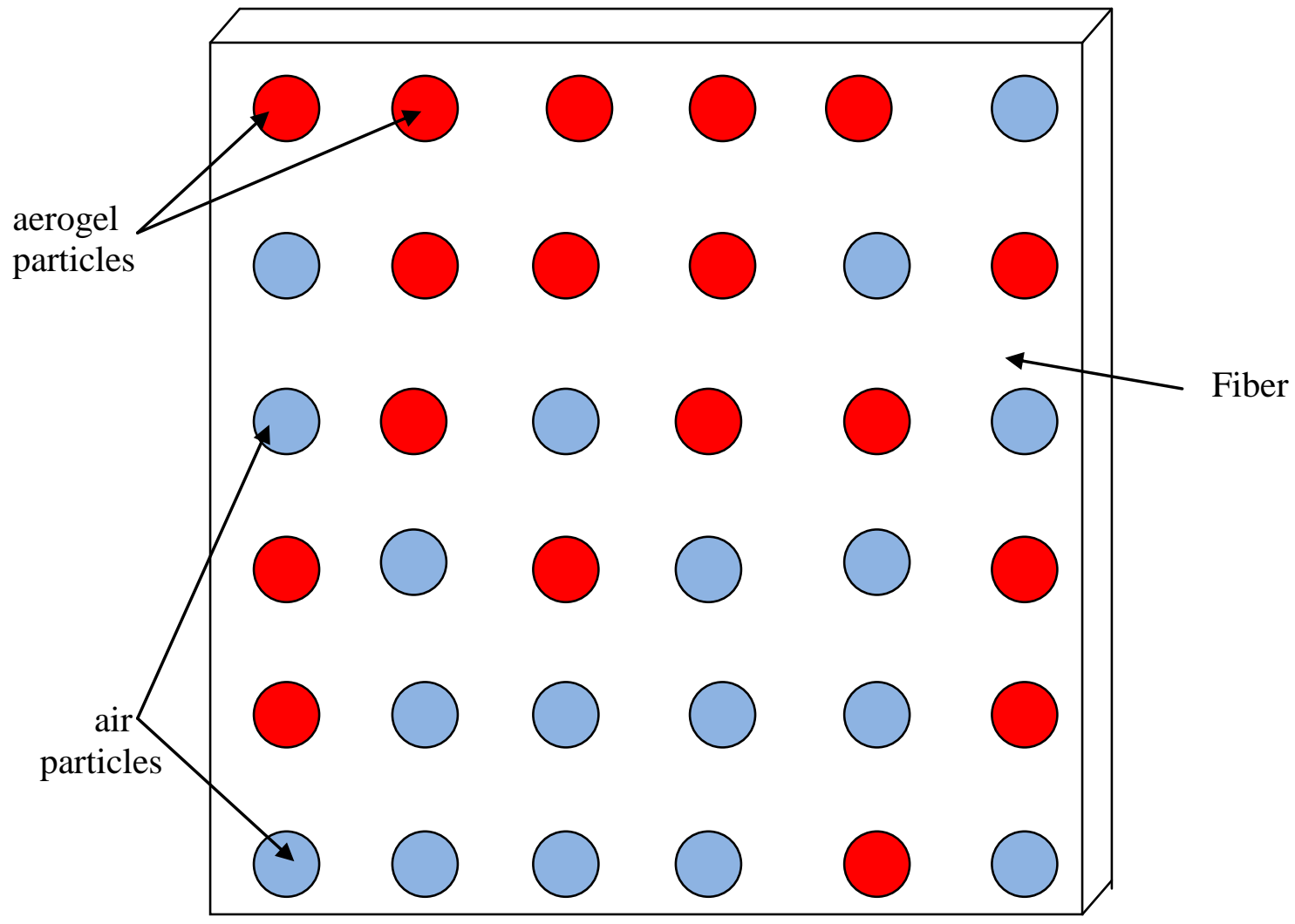

Figure 6.1: Schematic of the simplified flexible aerogel blanket structure 
For the purpose of this analysis, all the fibers of the aerogel composites were grouped together as a bulk of a volume connected in parallel.Under this simplification, the details of the distribution of the fibers through the flexible aerogel blanket, as well as the shape of the fibers does not enter in this simple finite element model [133].

\subsection{Finite Element Formulation}

The Fourier heat conduction equation was solved to evaluate the temperature distribution for the aerogel composite during the heating process and was given by:

$$
\rho C_{p} \frac{\partial T}{\partial t}=\left(k_{x} \frac{\partial^{2} T}{\partial x^{2}}\right)+\left(k_{y} \frac{\partial^{2} T}{\partial y^{2}}\right)+\left(k_{z} \frac{\partial^{2} T}{\partial z^{2}}\right)
$$

Equation 6.1

The relation between the heat flux per unit area vector and the temperature gradient:

$$
\left\{\begin{array}{l}
q_{x} \\
q_{y} \\
q_{z}
\end{array}\right\}=-\left\{\begin{array}{lll}
k_{x} & 0 & 0 \\
0 & k_{y} & 0 \\
0 & 0 & k_{z}
\end{array}\right]\left\{\begin{array}{l}
\partial_{T} / \partial_{x} \\
\partial_{T} / \partial_{y} \\
\partial T / \partial_{z}
\end{array}\right\} \quad \text { or } \quad q=-K \nabla_{T}
$$

The average value of the effective thermal conductivity of the flexible aerogel blankets from the heat conduction was obtained from three simulations from the different directions of the heat flux in unit cell [115]:

$$
k_{\text {cond }}=\frac{k_{x}+k_{y}+k_{z}}{3}
$$

Equation 6.3

The governing equations are solved using a FEM in 3D as well. Using the variational formulation, the Fourier equation can be rewritten as:

$$
\int_{V}^{[}\left[\frac{\partial T}{\partial t} \delta_{T}-\frac{\partial T}{\partial x} \frac{\partial \delta_{T}}{\partial x}-\frac{\partial T}{\partial y} \frac{\partial \delta_{T}}{\partial y}-\frac{\partial T}{\partial_{z}} \frac{\partial \delta_{T}}{\partial_{z}}\right] d V+\oint_{\Gamma_{e}} \delta T d S=0 \quad \text { Equation } 6.4
$$

In finite element modeling, it is convenient to use a natural coordinate system to 
represent the location of each node, orientation of each element and to apply loads and boundary conditions [134]. Natural coordinates are local coordinates in a dimensionless form which offer the convenience of having -1 to 1 for the limits of integration. The Cartesian coordinates $\mathrm{x}, \mathrm{y}, \mathrm{z}$ are related to the natural coordinates $\xi, \eta, \zeta$ through the shape function $N_{i}$ as the following:

$$
\begin{aligned}
x & =\sum_{i=i}^{I} N_{i}(\xi, \eta, \zeta) x_{i} \\
y & =\sum_{i=i}^{I} N_{i}(\xi, \eta, \zeta) y_{i} \\
z & =\sum_{i=i}^{I} N_{i}(\xi, \eta, \zeta) z_{i}
\end{aligned}
$$

where the shape function $N_{i}$ associated with each node can be expressed in terms of the product of three functions $F_{1}, F_{2}$ and $F_{3}$ :

$$
N_{i}=F_{1}(\xi, \eta, \zeta) \cdot F_{2}(\xi, \eta, \zeta) \cdot F_{3}(\xi, \eta, \zeta)
$$

Equation 6.6

For an eight node brick element, the shape function and the Jacobian matrix are expressed as the following:

$$
N_{i}=\frac{1}{8}\left(1+\xi_{i} \xi\right) \cdot\left(1+\eta_{i} \eta\right) \cdot\left(1+\zeta_{i} \zeta\right)
$$

Equation 6.7

where $\mathrm{i}=1,2, \ldots, 8$.

$$
J=\left[\begin{array}{lll}
\frac{\partial x}{\partial \xi} & \frac{\partial y}{\partial \xi} & \frac{\partial z}{\partial \xi} \\
\frac{\partial x}{\partial \eta} & \frac{\partial y}{\partial \eta} & \frac{\partial z}{\partial \eta} \\
\frac{\partial x}{\partial \zeta} & \frac{\partial y}{\partial \zeta} & \frac{\partial z}{\partial \zeta}
\end{array}\right]
$$


According to the equations 6.7 and 6.8 , the Jacobian matrix can be rewritten as:

$$
J=\left\{\begin{array}{lll}
\sum_{j=1}^{8} \frac{\partial N_{j}}{\partial \xi} x_{j} & \sum_{j=1}^{8} \frac{\partial N_{j}}{\partial \xi} y_{j} & \sum_{j=1}^{8} \frac{\partial N_{j}}{\partial \xi} z_{j} \\
\sum_{j=1}^{8} \frac{\partial N_{j}}{\partial \eta} x_{j} & \sum_{j=1}^{8} \frac{\partial N_{j}}{\partial \eta} y_{j} & \sum_{j=1}^{8} \frac{\partial N_{j}}{\partial \eta} z_{j} \\
\sum_{j=1}^{8} \frac{\partial N_{j}}{\partial \zeta} x_{j} & \sum_{j=1}^{8} \frac{\partial N_{j}}{\partial \zeta} y_{j} & \sum_{j=1}^{8} \frac{\partial N_{j}}{\partial \zeta} z_{j}
\end{array}\right\}
$$

Equation 6.9

The variational formulation leading to a finite element formulation after introduction of the shape factor $N_{j}(x, y, z)$ with nodal values $T_{j}$. The spatial variation of a dependent variable such as temperature may be expressed in terms of shape factors by:

$T \ll, y, z=\sum_{i=1}^{8} N_{j}(x, y, z) T_{j}$

Equation 6.10

The heat capacity, the thermal conductivity and the nodal vector of external heat fluxes matrices can be approximated for an arbitrary element by [135]:

$$
\begin{aligned}
& C^{(e)}=\int_{-1}^{1} \int_{-1}^{1} \int_{-1}^{1} N^{T} N|J| d \xi d \eta d \zeta \\
& K^{(e)}=\int_{-1}^{1} \int_{-1}^{1} \int_{-1}^{1} B|J| d \xi d \eta d \zeta \\
& F^{(e)}=\int_{-1}^{1} \int_{-1}^{1} q N^{T}|J| d \xi d \eta
\end{aligned}
$$

where $B=\left\{\frac{\partial}{\partial x}, \frac{\partial}{\partial y}, \frac{\partial}{\partial z}\right\}^{T} N \mathbb{\&}, y, z=J^{-1}\left\{\frac{\partial}{\partial \xi}, \frac{\partial}{\partial \eta}, \frac{\partial}{\partial \zeta}\right\}^{T} N \boldsymbol{\in}, \eta, \zeta_{-}^{-}$

In the finite element software, the conductivity equation becomes:

$$
C\{T\}+K\{T\}=\{F\}
$$

Equation 6.14 
where $\mathrm{C}$ is the heat capacity matrix, $\mathrm{K}$ the thermal conductivity matrix and $\mathrm{F}$ the nodal vector of external heat fluxes.

Galerkin approximation formula [136] can be used to approximately determine the time derivative of temperature $\{T\}$. This gives the possibility to rewrite the matrix equation as follow:

$$
\begin{aligned}
& \bar{K}=C_{n}+\frac{2}{3} \Delta_{t_{n+1}} K_{n+1} \\
& \{\bar{F}\}=\left\lceil C_{n}-\frac{1}{3} \Delta_{t_{n+1}} K_{n}\right]\{T\}+\Delta_{t_{n+1}}\left[\frac{2}{3}\{F\}_{n+1}+\frac{1}{3}\{F\}_{n}\right] \\
& \bar{K}\{T\}_{n+1}=\{\bar{F}\}_{n, n+1}
\end{aligned}
$$

The terms of the know solution at time $t_{n}$ is used to express, the solution at $t=t_{n+1}$. Knowing the initial conditions at $\mathrm{t}=0$, the solutions of the matrix equation can be found marching in time.

\subsection{Initial and Boundary conditions}

For transient processes of heat conduction and heat convection, it is necessary to define the initial distribution of temperature and heat transfer coefficient in the flexible aerogel blanket samples. The initial value of the temperature field of the finite size of the blanket in orthogonal Cartesian coordinate system is given by:

$$
\left.T(\vec{r}, t)\right|_{t=0}=T(\vec{r}, 0)=T_{0}(\vec{r})
$$

Equation 6.16

Where $\vec{r}$ the radius vector of some points is defined as: $\vec{r}=x \vec{r}+y \vec{j}+z \vec{k}$ The initial heat transfer coefficient of the aerogel composite sample is reported in the 
following:

$\left.h(\vec{r}, t)\right|_{t=0}=h(\vec{r}, 0)=h_{0}(\vec{r})$

Equation 6.17

Consider a domain $\Omega$ in 3-D space on the a surface of an aerogel blanket sample having a boundary $\Gamma$ which consist of disjoint portions $\Gamma_{i}$ such that:

$\Gamma=\Gamma_{1} \cup \Gamma_{2} \cup \Gamma_{3}$

Equation 6.18

In general, the boundary conditions can be classified in three types[137]. The first is the Dirichlet or essential boundary conditions for which the temperature values are given on surface $\Gamma_{1}$ as:

$\left.T(\vec{r}, t)\right|_{\Gamma_{1}}=T_{\Gamma}(\vec{r}, t)$

Equation 6.19

The second type is the Newman or natural boundary condition which is expressed in the following:

$\left.n \cdot q(\vec{r}, t)\right|_{\Gamma_{2}}=-\left.n \cdot K(\vec{r}) \cdot \nabla T\right|_{\Gamma_{2}}=q_{\Gamma}(\vec{r}, t)$

Equation 6.20

Where $q_{\Gamma}(\vec{r}, t)$ is the heat flux per unit area on $\Gamma_{2}$ surface and $\mathrm{n}$ is the normal unit vector to the surface.The last type is the conditions of Cauchy or Riehmann which is expressed as:

$$
\left.n \cdot q(\vec{r}, t)\right|_{\Gamma_{3}}=-\left.n \cdot K(\vec{r}) \cdot \nabla T\right|_{\Gamma_{3}}=\left.h_{\Gamma}(\vec{r}, t) \overrightarrow{\mathbf{l}}\right|_{\Gamma_{3}}-T_{\infty}(\vec{r}, t) \text { - }_{-}
$$

Equation 6.21

These initial and boundary conditions are used to supplement the governing model equations mentioned in the previous section of this chapter. 


\subsection{Conduction Elements used by ANSYS}

ANSYS offers a number of 3-D thermal solid elements with the ability to conduct heat between their nodes. These solid elements include PLANE78, SOLID 70, SOLID 87 and SOLID 90. To analyze 3-D heat conduction problems, SOLID 70 and SOLID 90, as shown in figure $6.2(a, b)$, are commonly used.

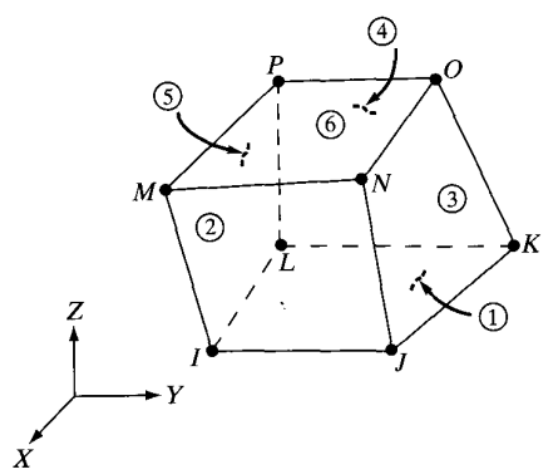

(a)

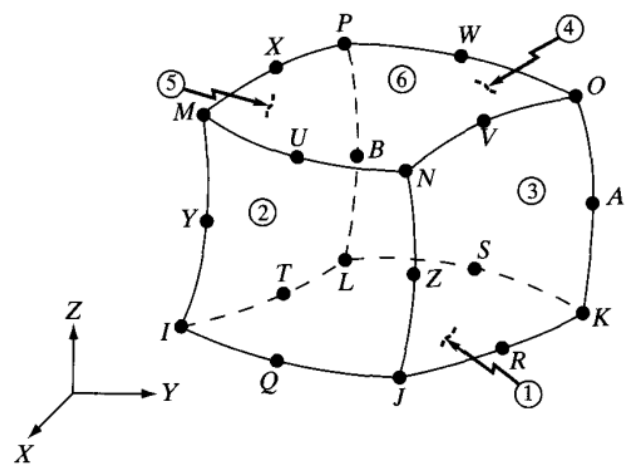

(b)

Figure 6.2: (a) Solid 70; (b) Solid 90 [134]

SOLID 70 is an eight node brick element that has at each node, a degree of freedom, which is temperature. Heat generation rates can also be applied at the nodes. Convection and heat fluxes at the element's face are the required input information. In figure 6.2, the element's faces are shown by circled numbers. Output data include nodal temperatures and element data, such as thermal gradient and thermal fluxes. SOLID 90 is defined by its twenty nodes quadrilateral thermal solid element. It is more accurate than the SOLID 70 but requires more time solution. The computational process of SOLID 90 element is more complex than that of the SOLID 70 element because of the large amount of numerical integration of elemental matrices involved. In addition, SOLID 90 is well suited for the analysis of heat transfer problems with curved boundaries. Each node of this element uses temperature as a single degree of 
freedom. Input and output data for this element are similar to the data format of SOLID 70 elements.

\subsection{Mesh Design}

Before initiating the meshing process, the element attributes and element size need to be specified. The element attributes provide information related to the element type(s), real constants and material properties. The element size is defined by either a global element size or by specifying the number of element divisions along a boundary line.

The desired size level of the mesh ranges from 1 (fine) to 10 (coarse), defaults to 6 . Two methods were used to generate the mesh of the geometry under consideration: Free or automatic meshing and mapped meshing [134].

The free meshing concept is valid in 1-D, 2-D and 3-D problems. The solid model entities meshed with this option use either mixed area element shapes or all triangular area elements. After the selection of the line number, areas are used as input and the mesh is generated automatically. The desired mesh density and mesh shape can be selected. The mapped meshing concept is valid only for models utilizing 2-D and 3-D elements. Although mapped meshing uses all quadrilateral area elements and all hexahedral volume elements, not every area can be mashed meshed. For an area to be mapped meshed. It has to be "regular". i.e. it needs to have 3 or 4 sides, equal number of elements on opposite sides and even numbers of elements for three sided areas. To mapped mesh irregular areas, it is mandatory to reduce the number of sides to the required numbers. This can be achieved by combining many sides into a single side. This process is called "concatenating" lines. 
The mesh density can be controlled by using the following tools:

- Smart sizing, Global element sizing and Default sizing on a global level

- Keypoint sizing, Lines sizing and Area sizing on a local level

Figure 6.3 shows the finite element discretization of a fiber of a flexible aerogel blanket.

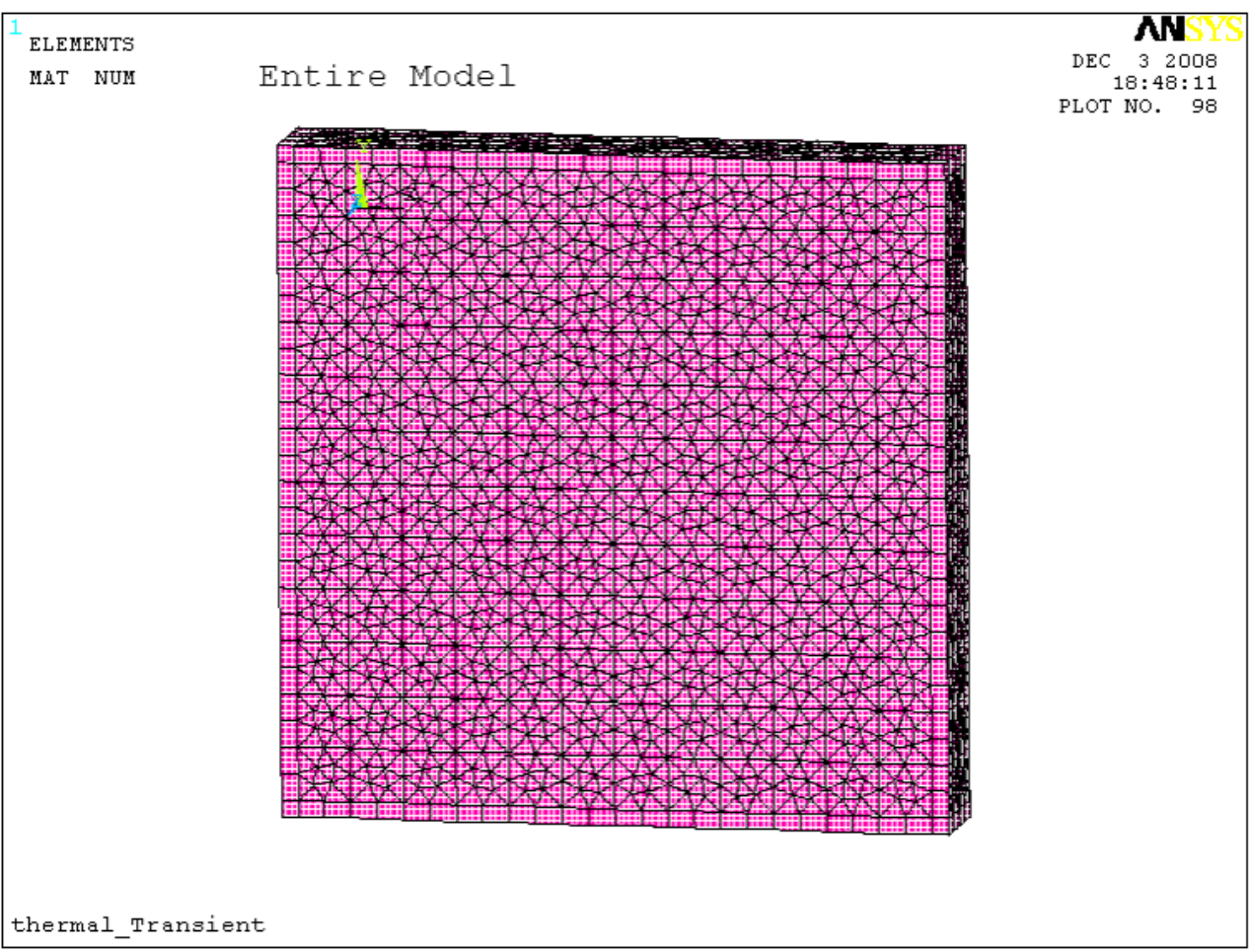

Figure 6.3: FEM mesh of an aerogel blanket

\subsection{Modeling the heat transfer of flexible aerogel blanket}

A computational model using the FEM implemented with ANSYS software was used to study the heat transfer of flexible aerogel blankets [138-140]. In this model, the flexible aerogel blanket loses aerogel particles as a result of degradation. As the degradation process of the aerogel composite occurs, the aerogel particles are replaced by air particles. When the insulation blanket reaches its final stage of degradation, it will be 
surrounded by air particles only. The evolution of the flexible aerogel blanket degradation was studied using the influence of the aerogel particle volume fraction. Five cases, in which the volume fraction of the aerogel particles varies from 0 to 0.4 , are investigated. The volume fraction of aerogel particles has the following value:

- 0 for the first case

- 0.05 for the second case

- 0.15 for the third case

- $\quad 0.25$ for the fourth case

- 0.4 for the fifth case

This volume fraction of the aerogel particles range was selected based on results obtained in the quantative analysis of the SEM pictures of the flexible aerogel blanket in chapter 5 . In this FEM, the heat flow is assumed to be 1-D. The bottom surface of the insulation blanket is exposed to a hot plate and its top surface to air. The temperature of the hot plate is set up to $200^{\circ} \mathrm{C}$ and the room temperature $25^{\circ} \mathrm{C}$. The bottom and top surface thermal loads of the flexible aerogel blanket are shown in figure 6.4 ( $a$ and $b$ ).

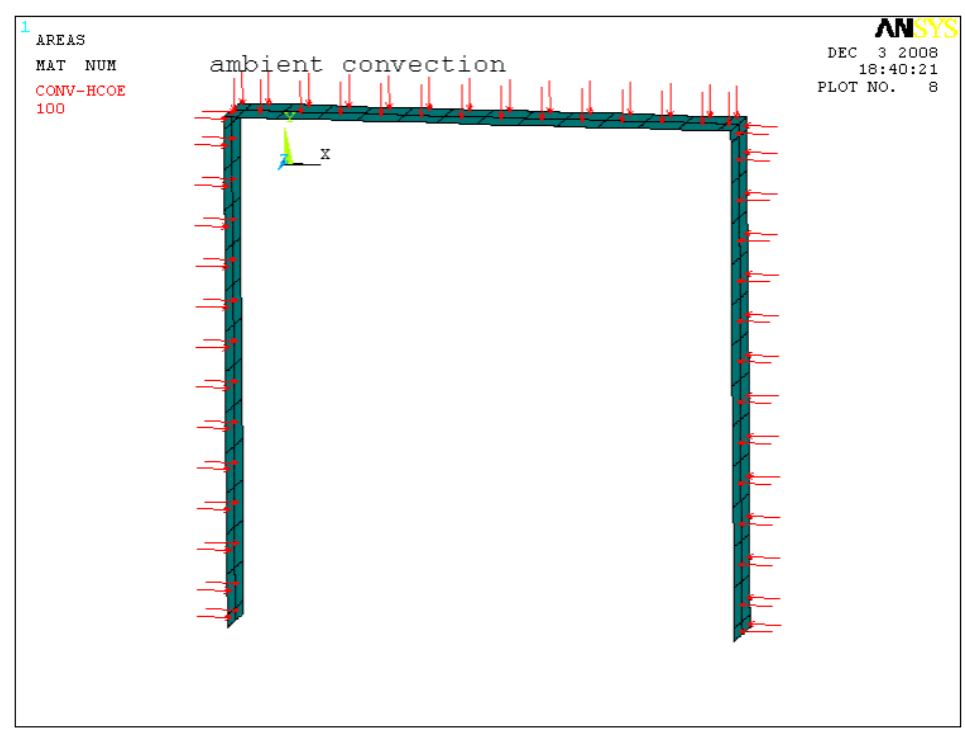

(a) 


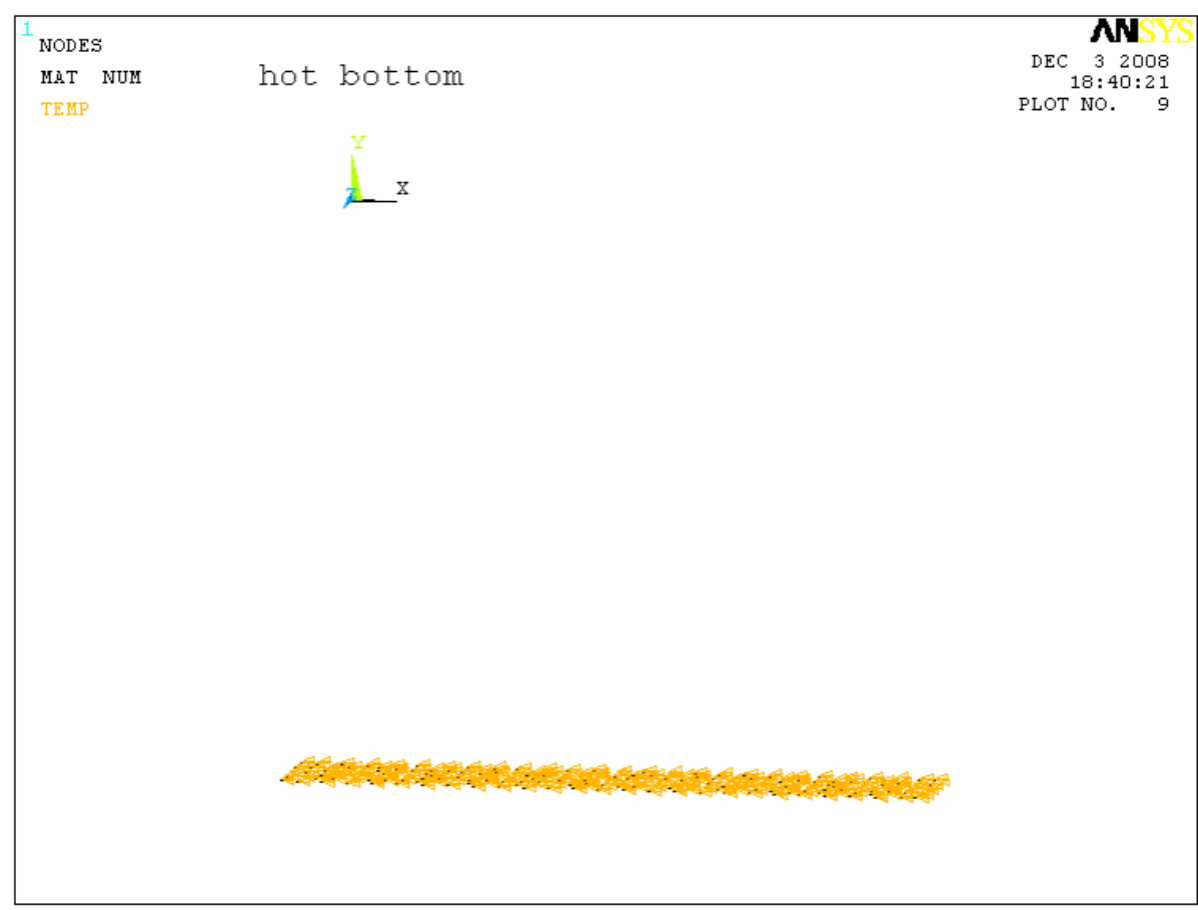

(b)

Figure 6.4: Thermal loads of an aerogel blanket
(a) Top surface (b) Bottom surface

\subsection{Results and Discussions of the FEM Model}

For the complete degradation of the flexible aerogel blanket (fiber with no aerogel particles), the FE model discretization of the fiber with air particles, the temperature distribution and the vector plot of nodal heat flux over the matrix are shown in figures 6.5 ( $\mathrm{a}$ and $\mathrm{b}$ ), 6.6, and 6.7 respectively.

The FE model stimulation results which include the meshed fiber and particles, the temperature and heat flux distributions for different aerogel particle volume fractions (from case 2 to case 5) are shown in figures 6.9-6.20. 


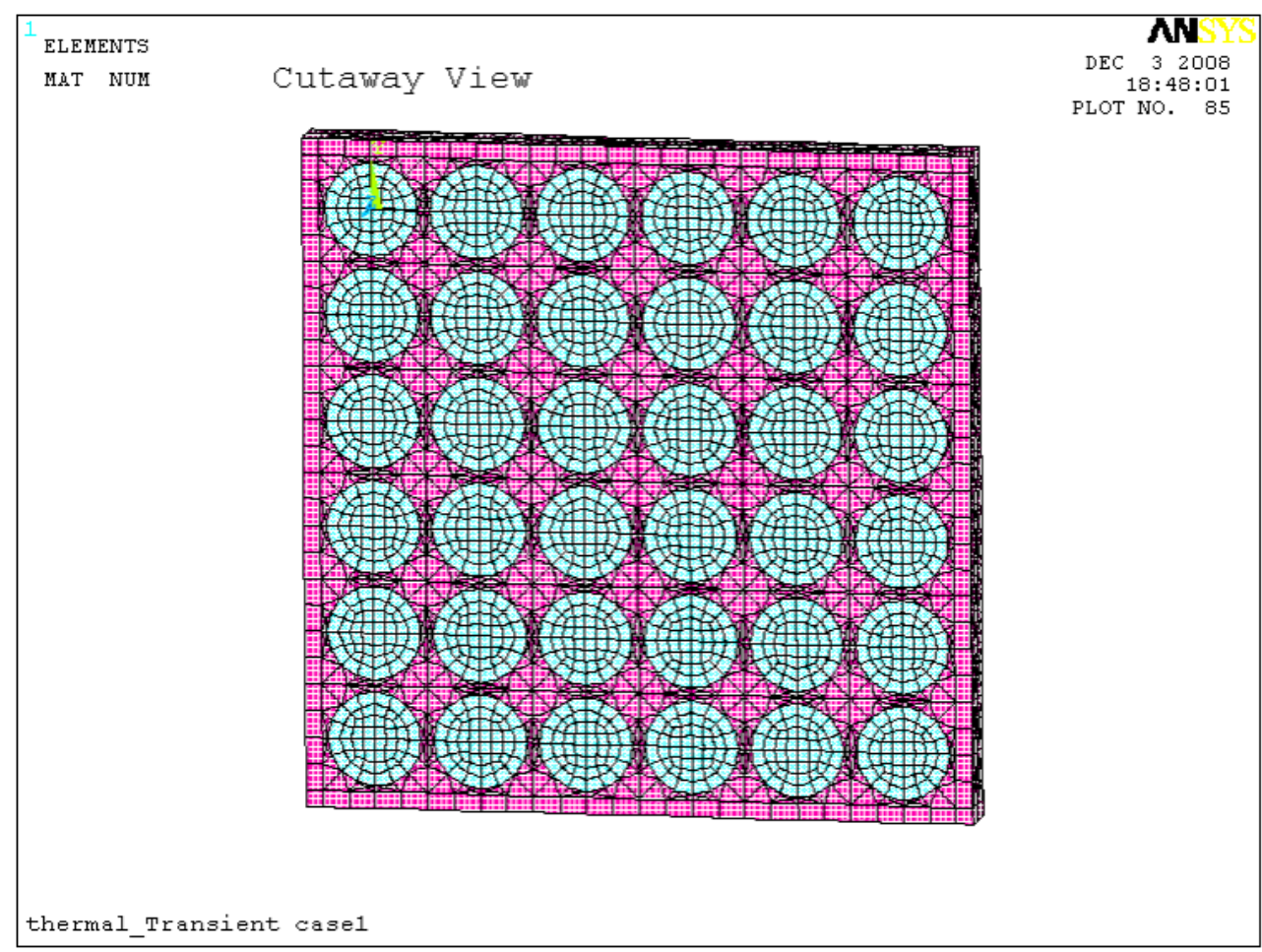

(a)

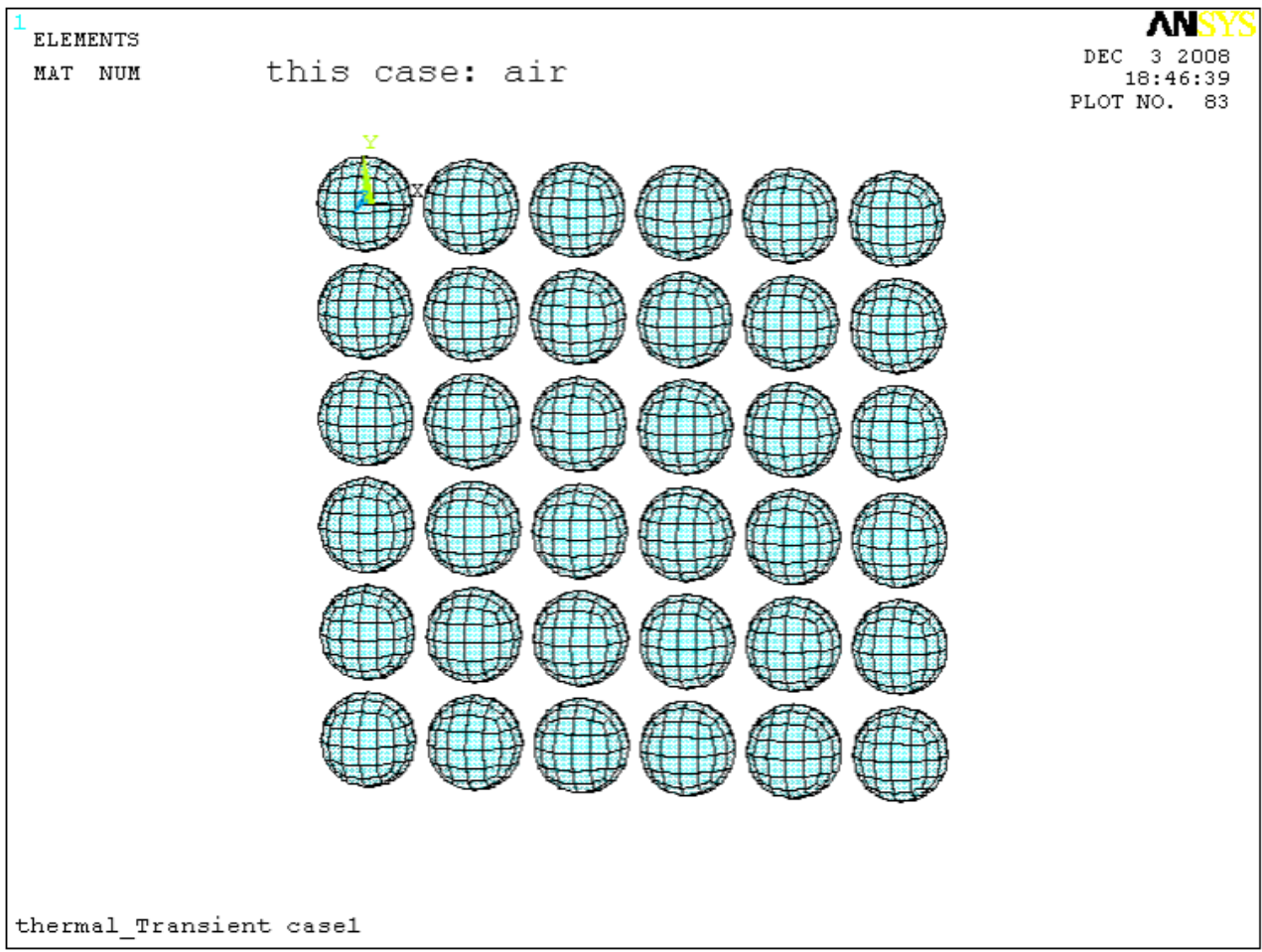

(b)

Figure 6.5: FEM mesh of: (a) fiber with no aerogel and (b) air particles 


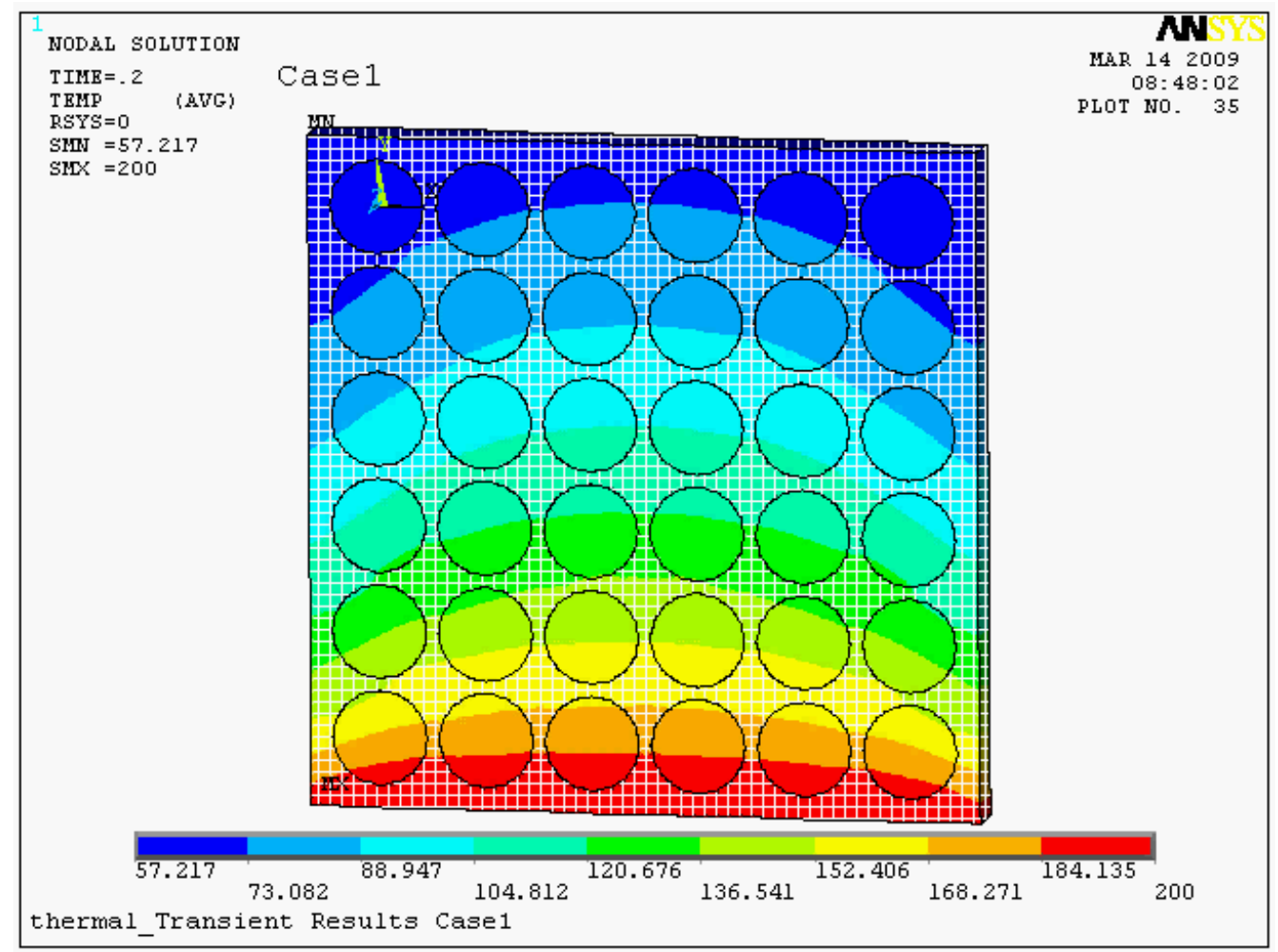

Figure 6.6: Distribution of temperature over fiber with no aerogel

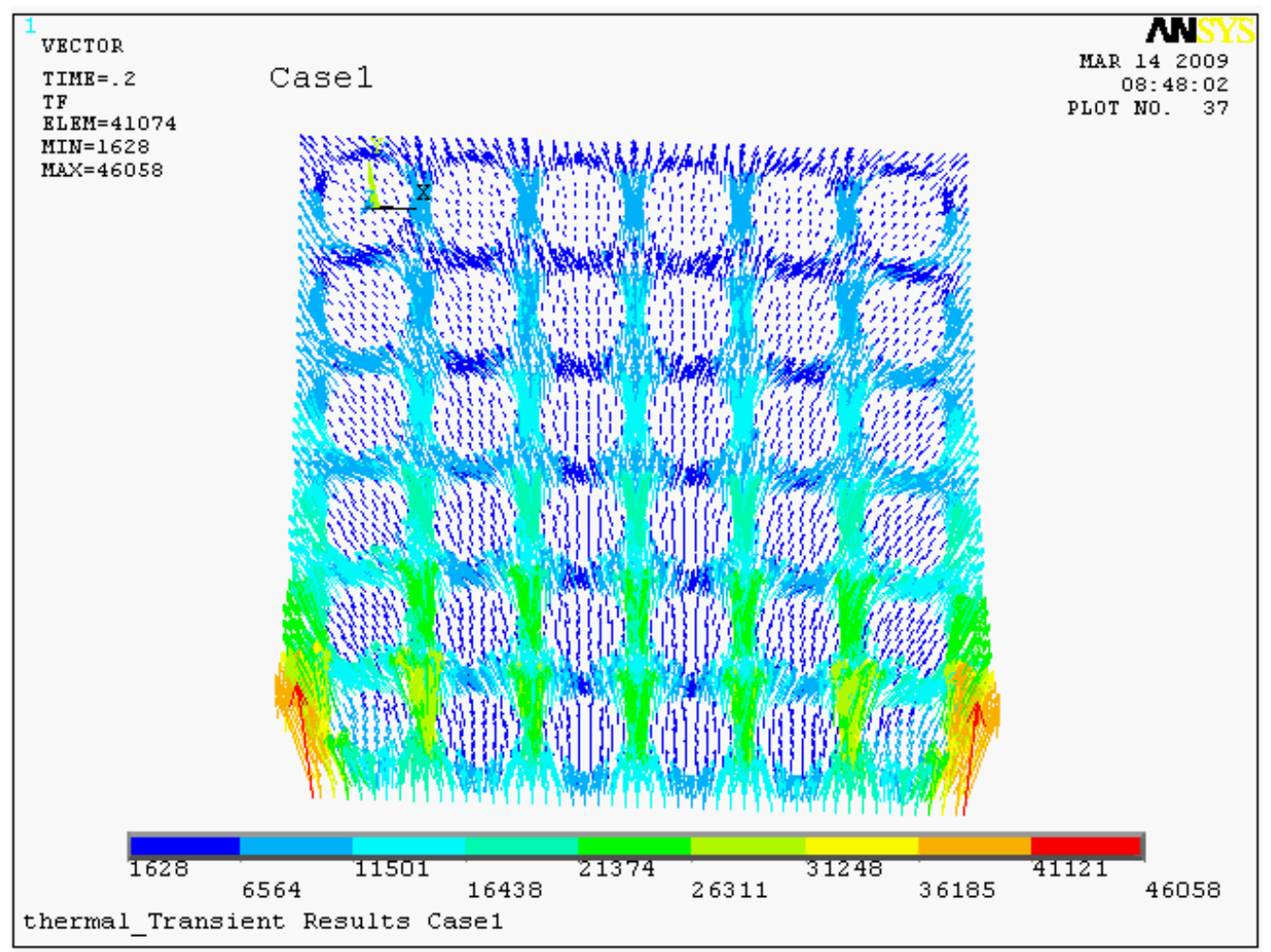

Figure 6.7: Distribution of heat flux over fiber with no Aerogel 


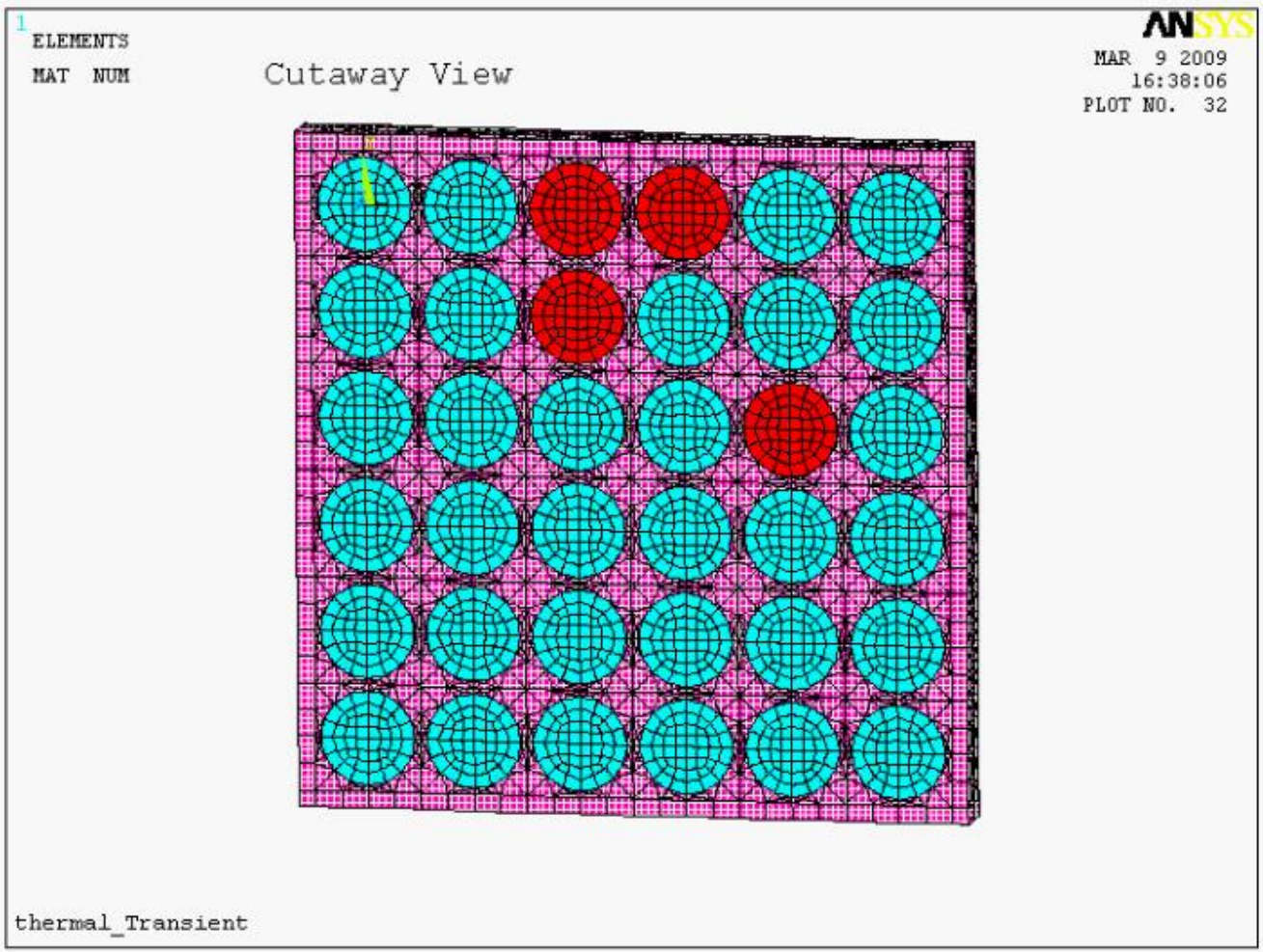

(a)

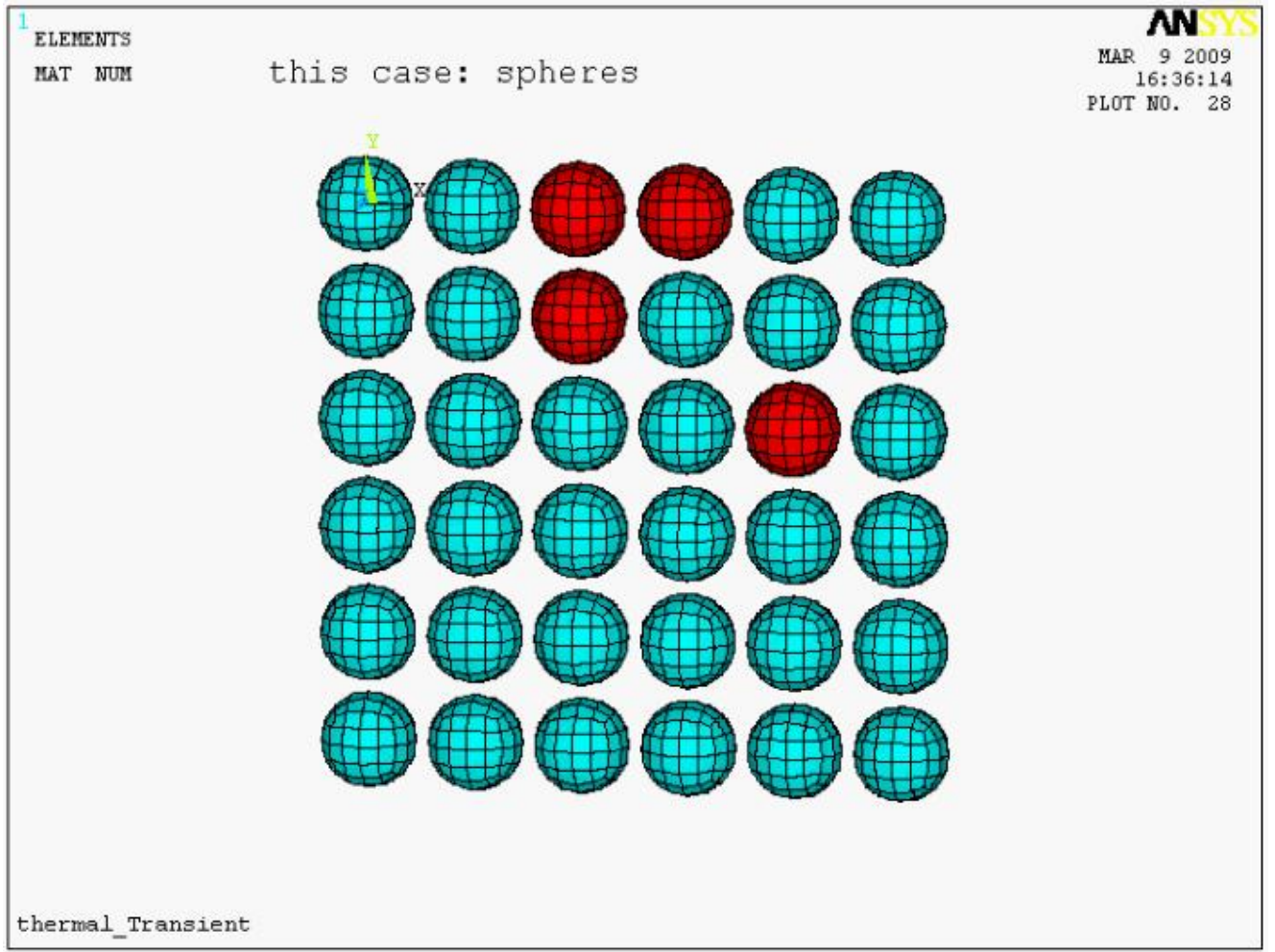

(b)

Figure 6.8: FEM mesh of: (a) fiber with 0.05 VF Aerogel

(b) aerogel (red) and air (blue) particles 


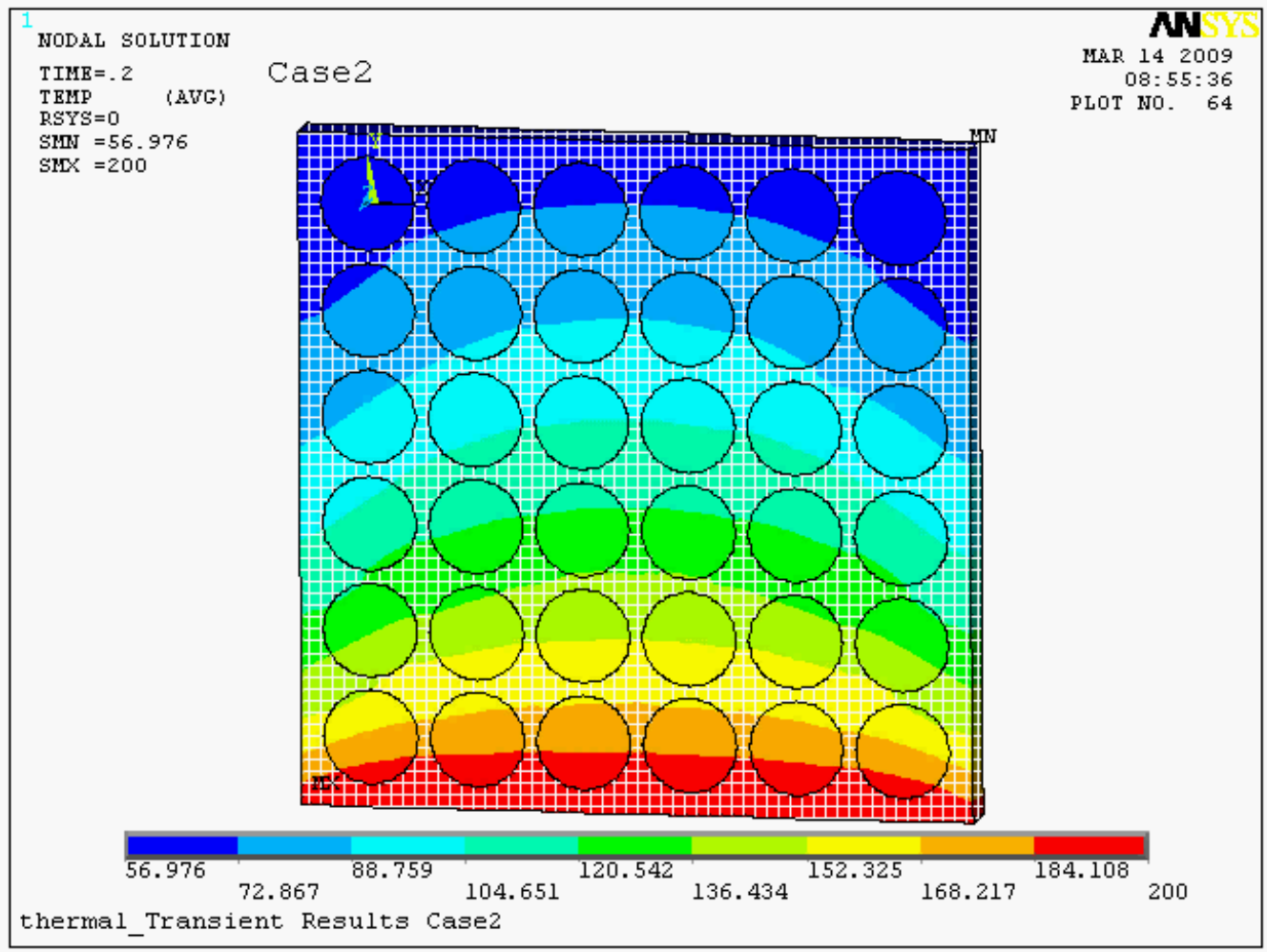

Figure 6.9: Distribution of temperature over fiber with $0.05 \mathrm{VF}$ aerogel

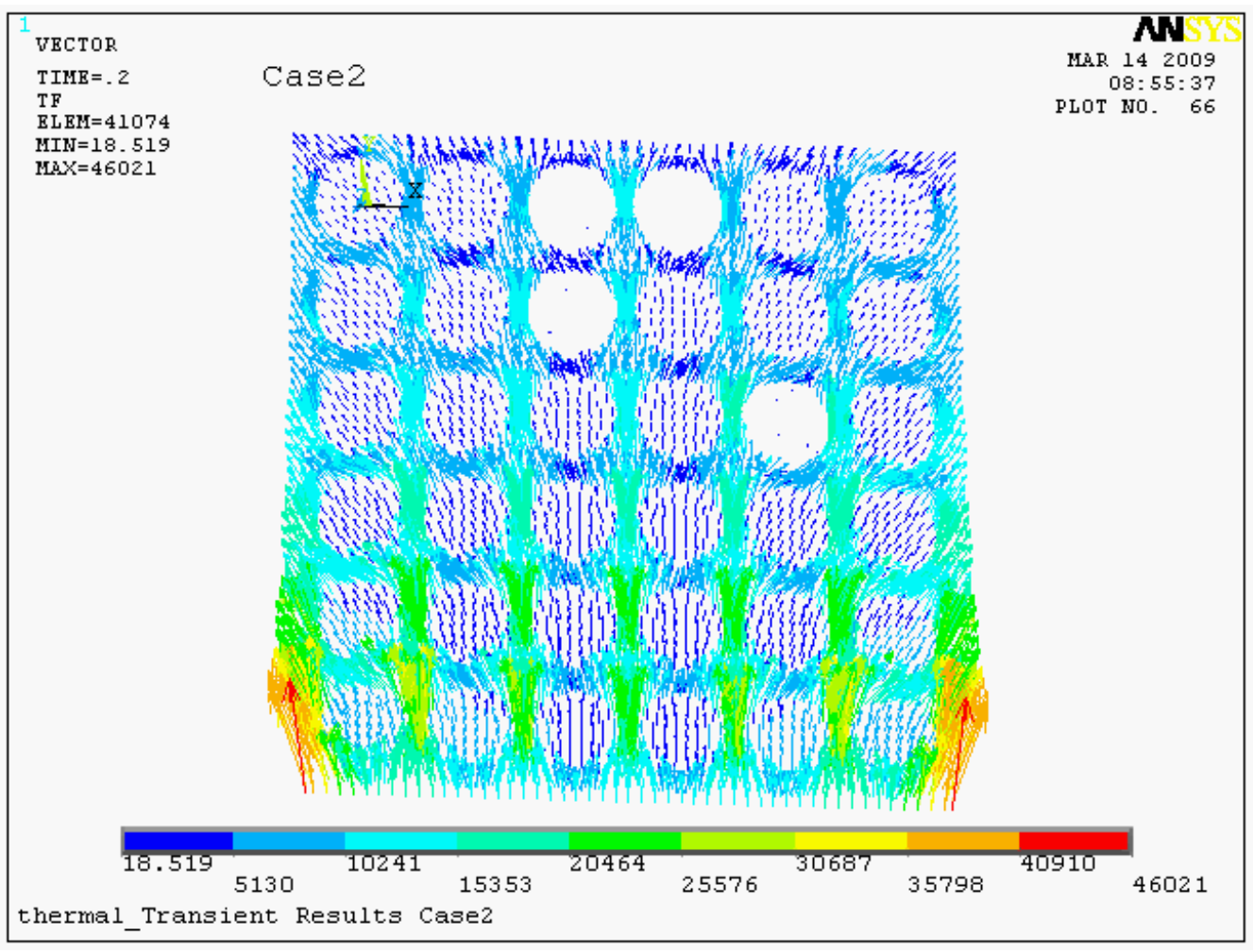

Figure 6.10: Distribution of heat flux over fiber with 0.05 VF Aerogel 


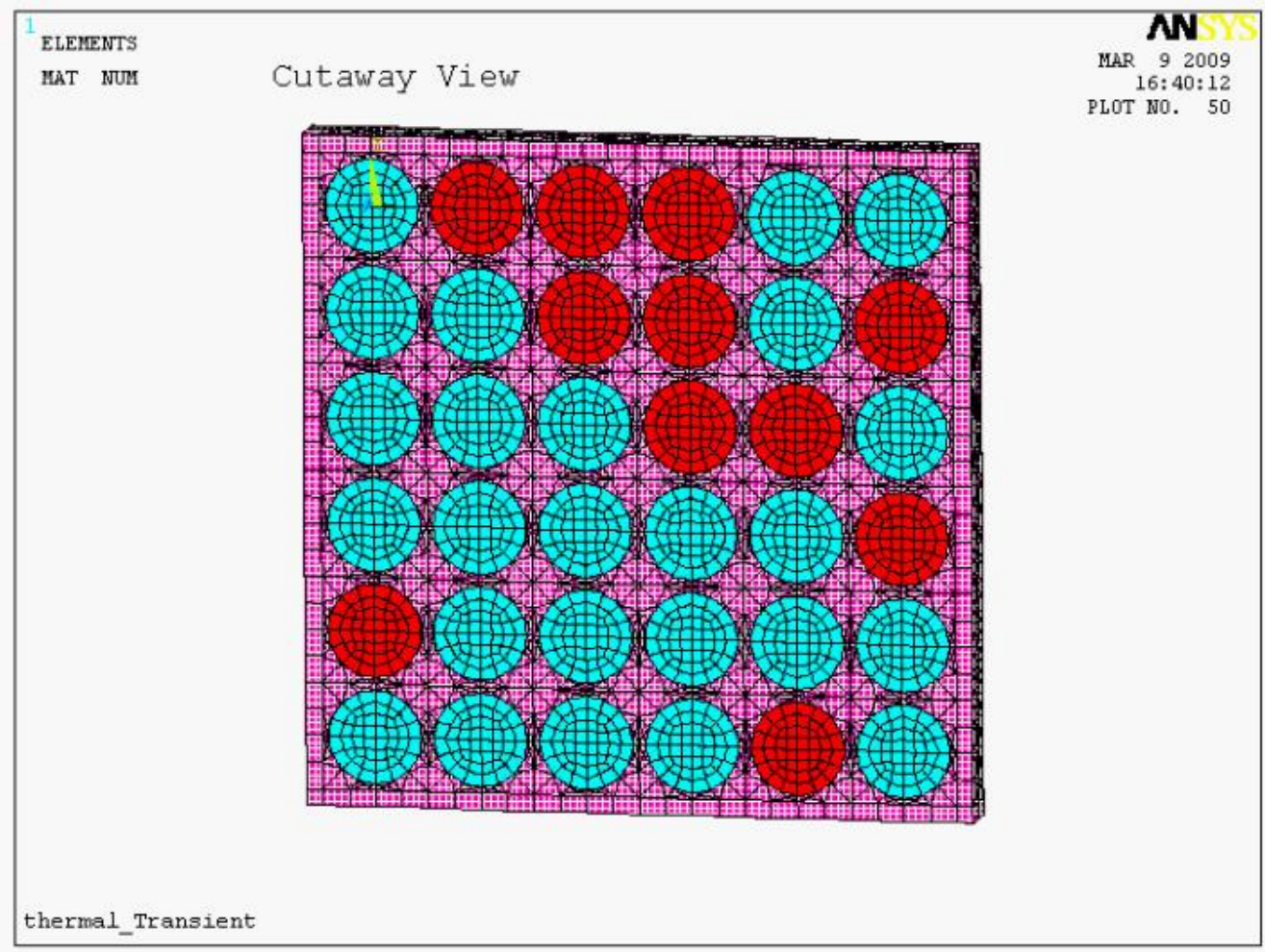

(a)

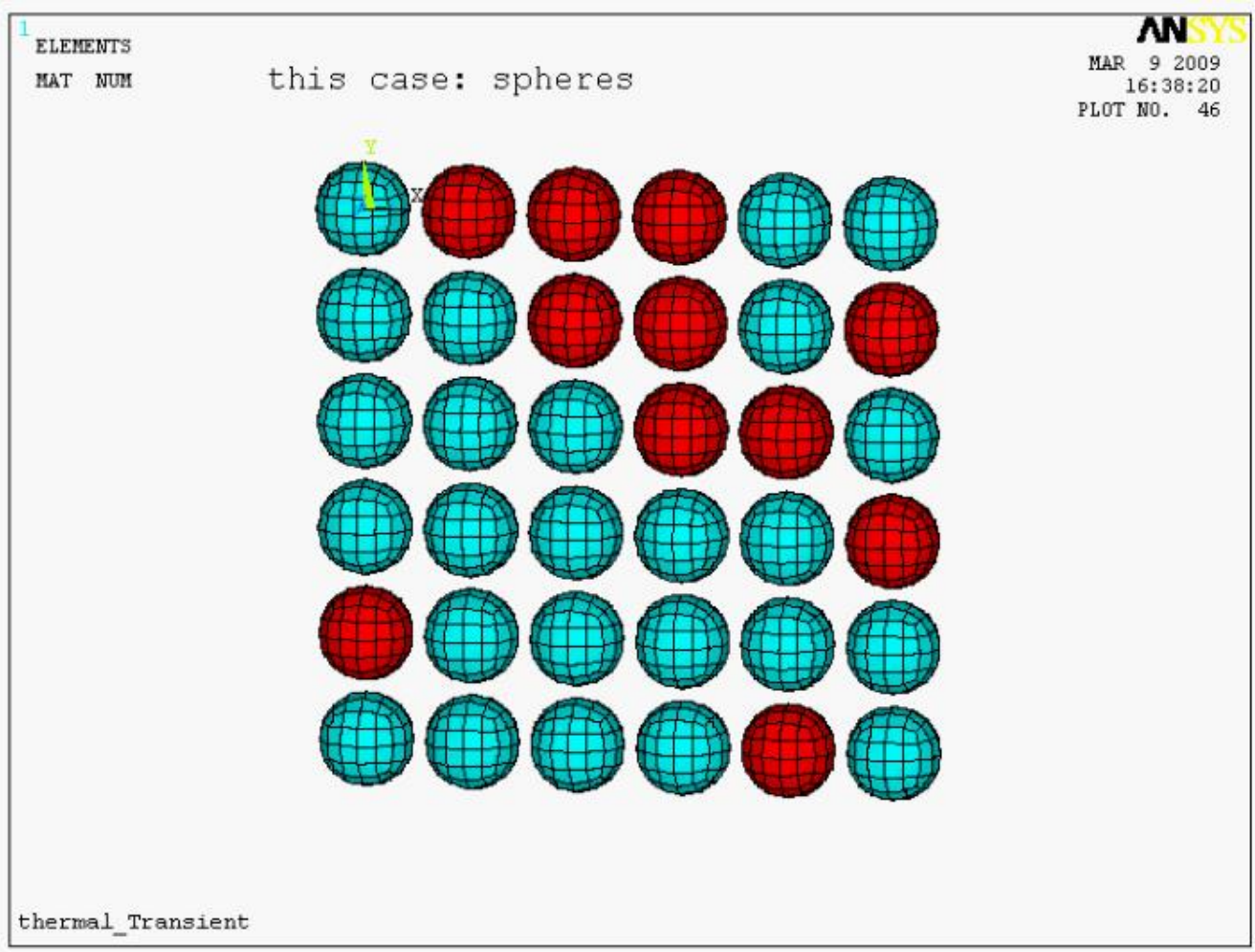

(b)

Figure 6.11: FEM mesh of: (a) fiber with $0.15 \mathrm{VF}$ aerogel

(b) aerogel (red) and air (blue) particles 


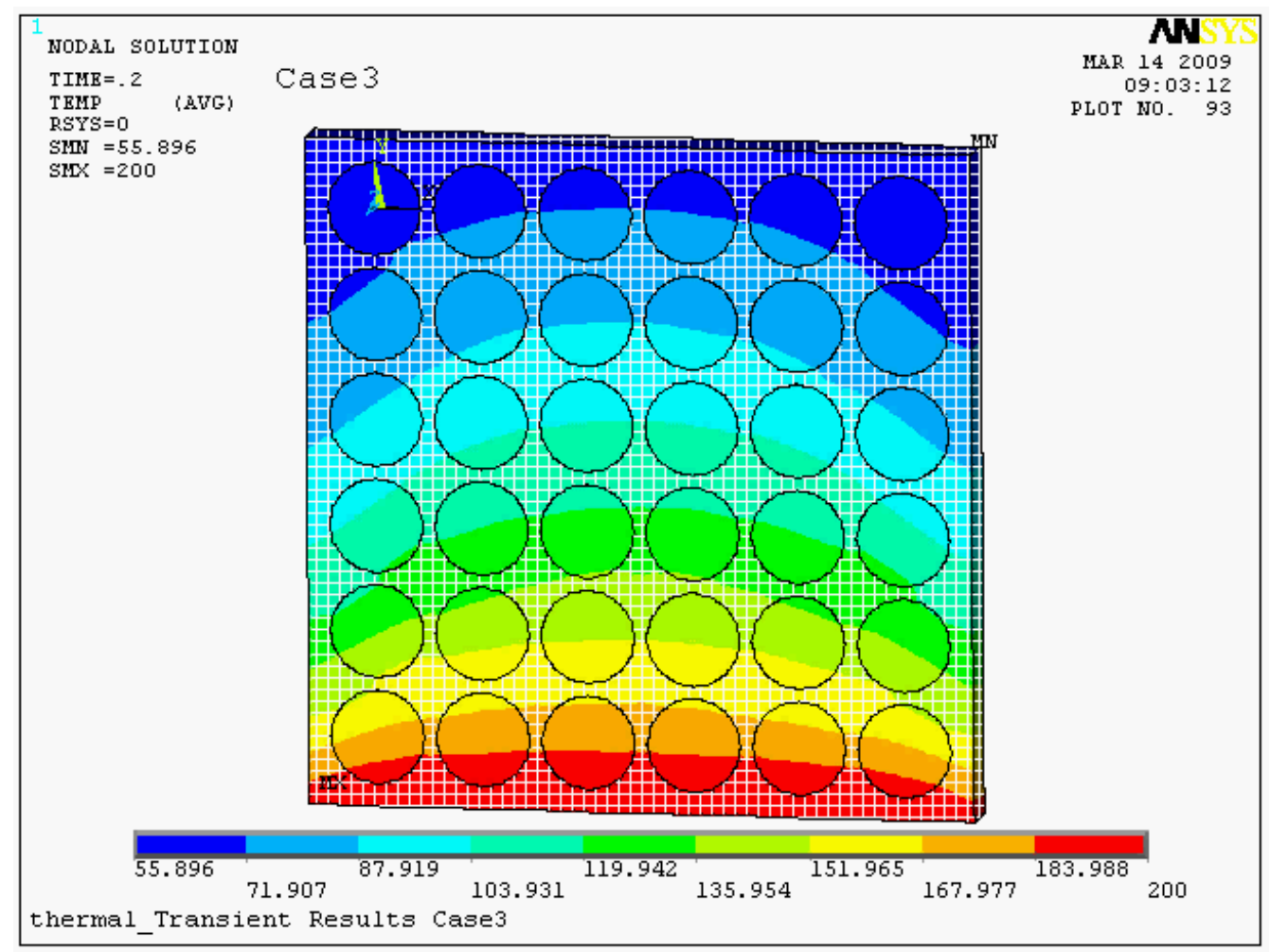

Figure 6.12: Distribution of temperature over fiber with $0.15 \mathrm{VF}$ aerogel

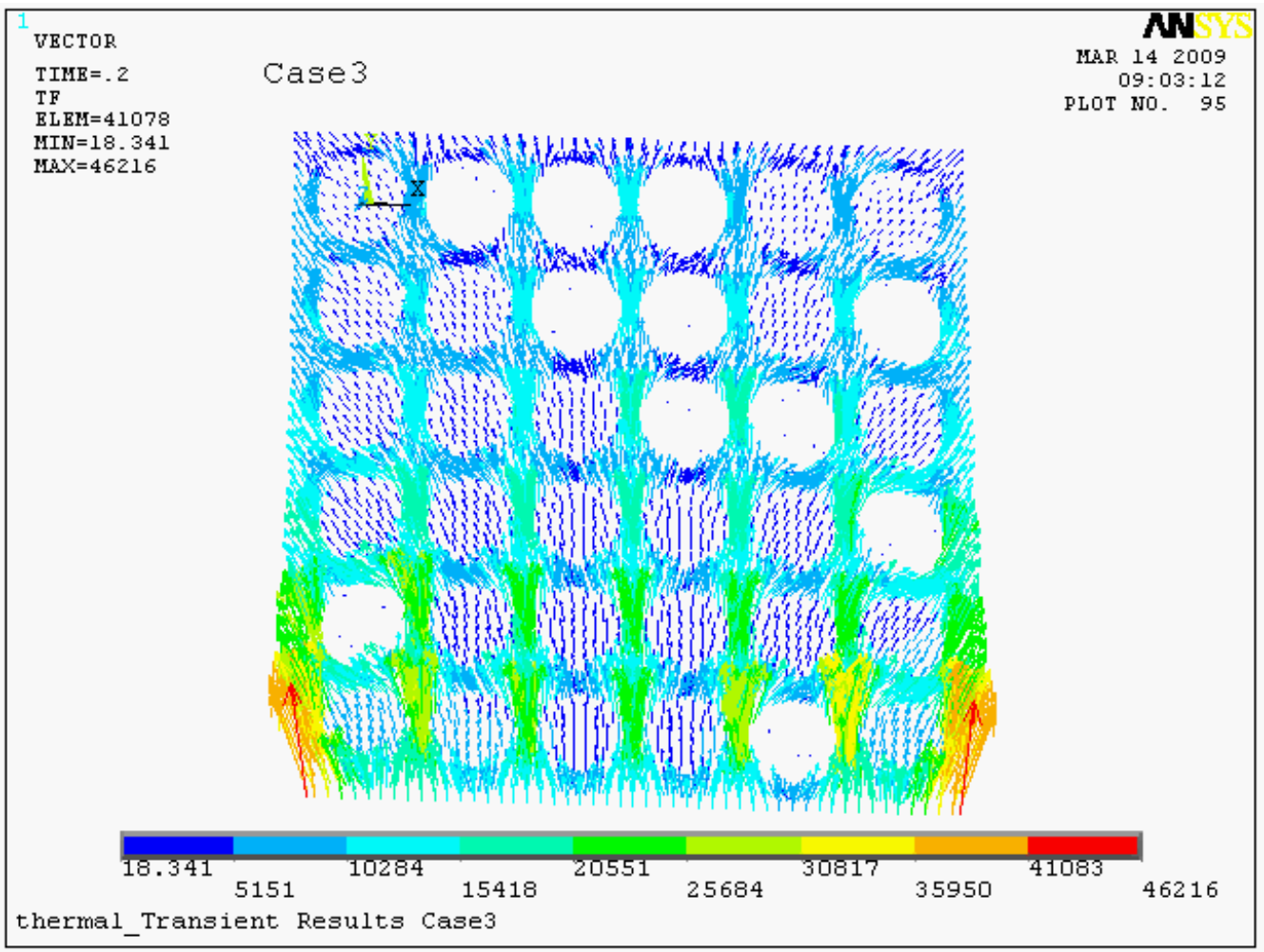

Figure 6.13: Distribution of heat flux over fiber with 0.15 VF Aerogel 


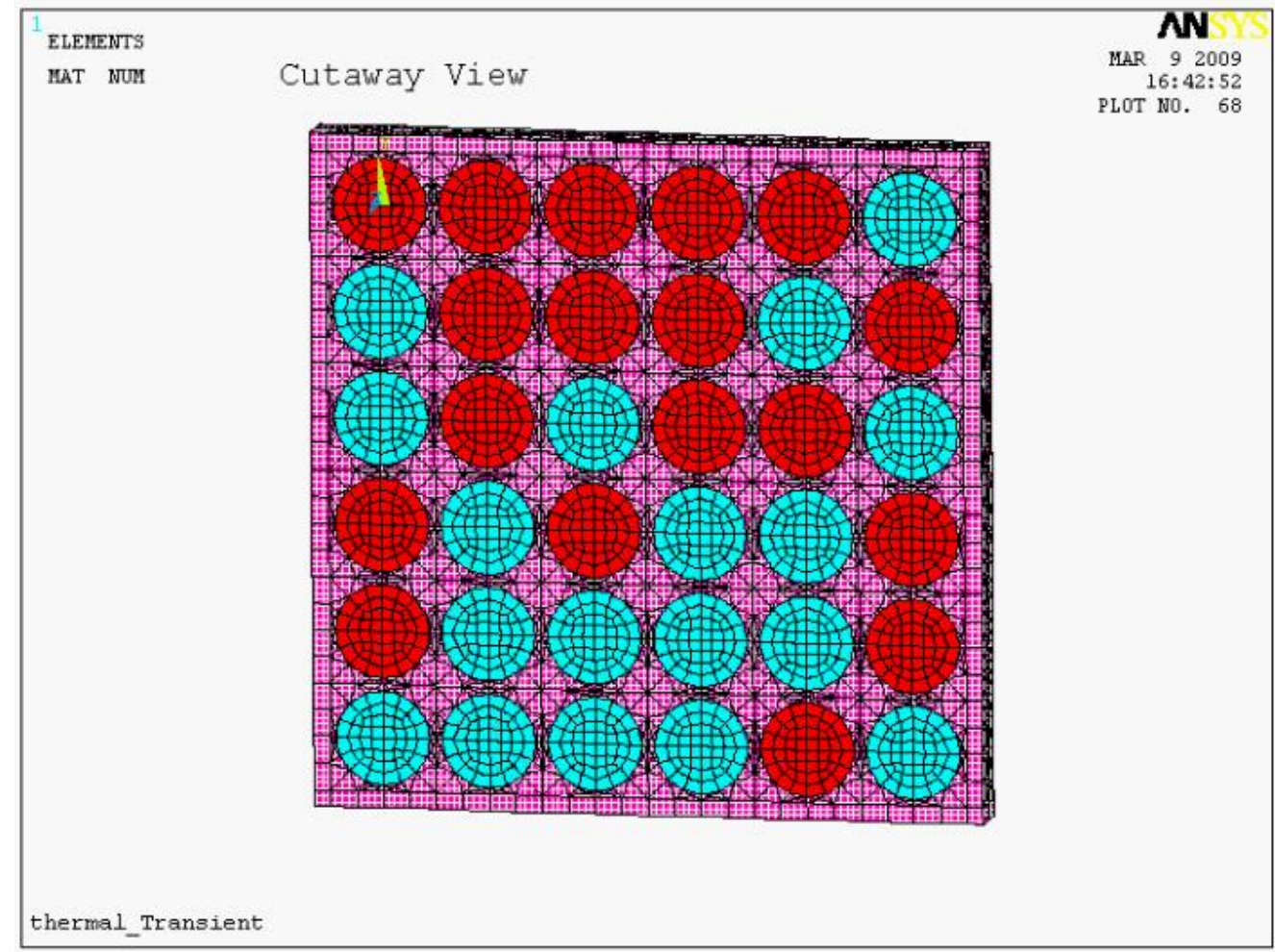

(a)

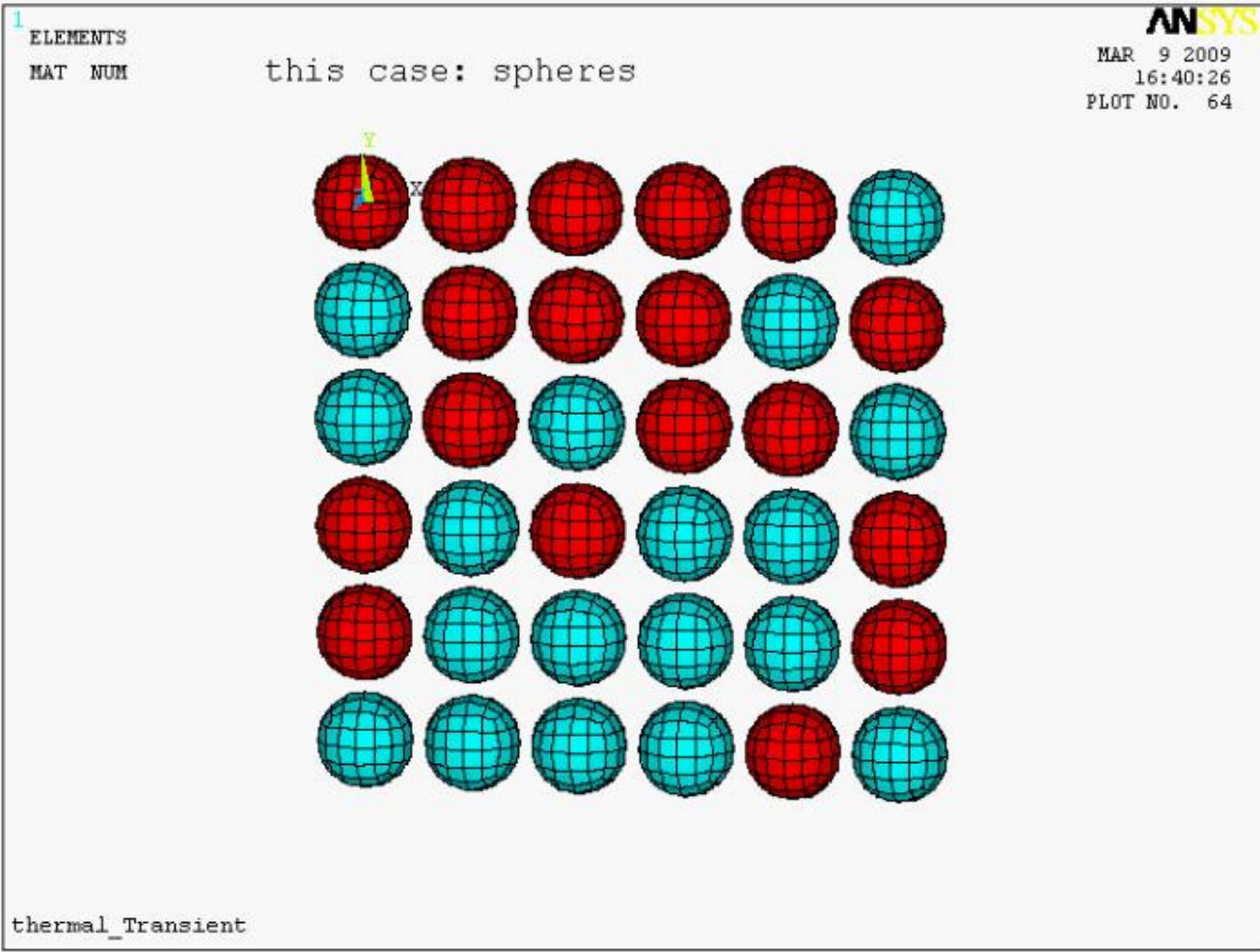

(b)

Figure 6.14: FEM mesh of: (a) fiber with $0.25 \mathrm{VF}$ aerogel

(b) aerogel (red) and air (blue) particles 


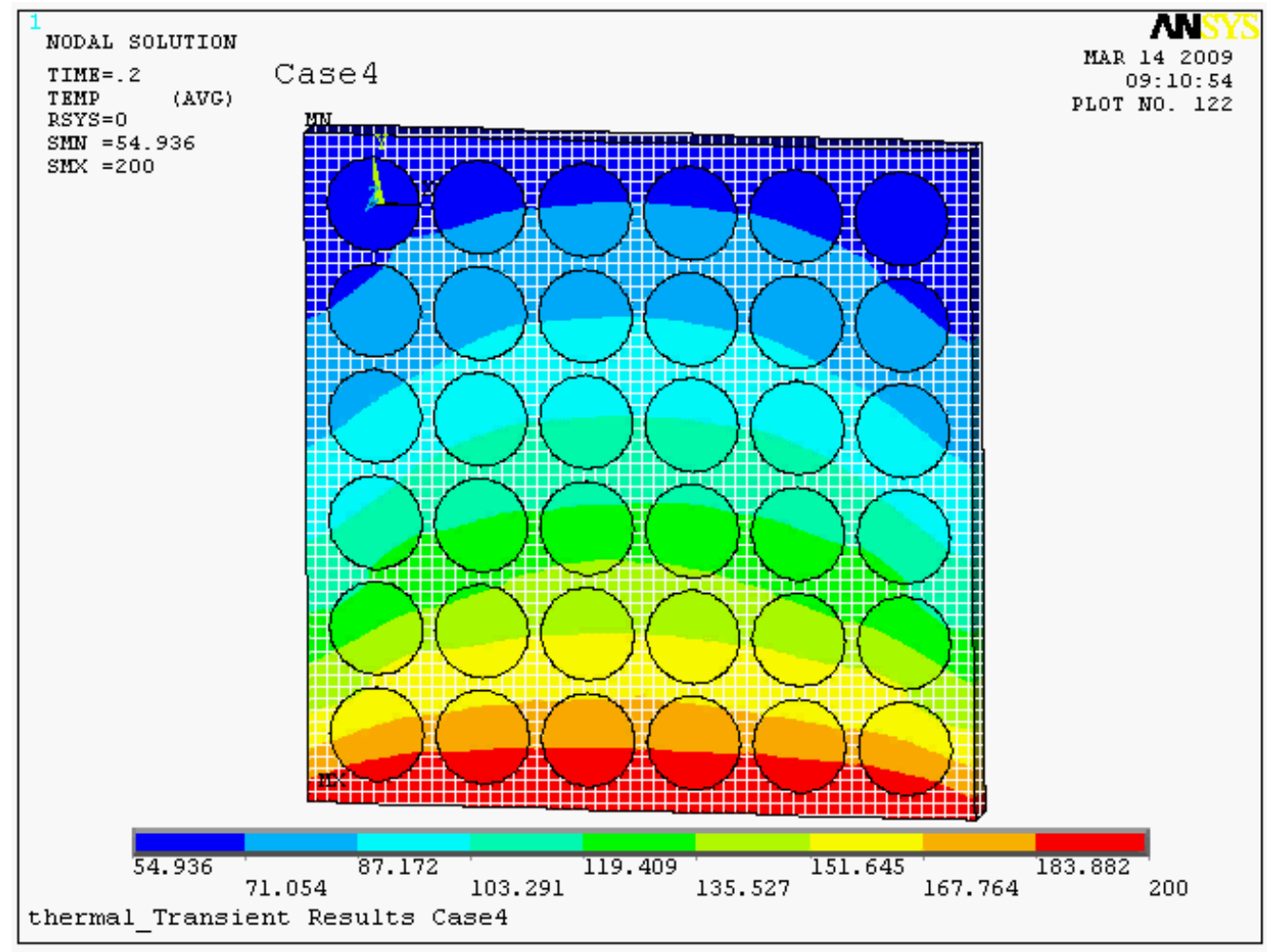

Figure 6.15: Distribution of temperature over the fiber with $0.25 \mathrm{VF}$ aerogel

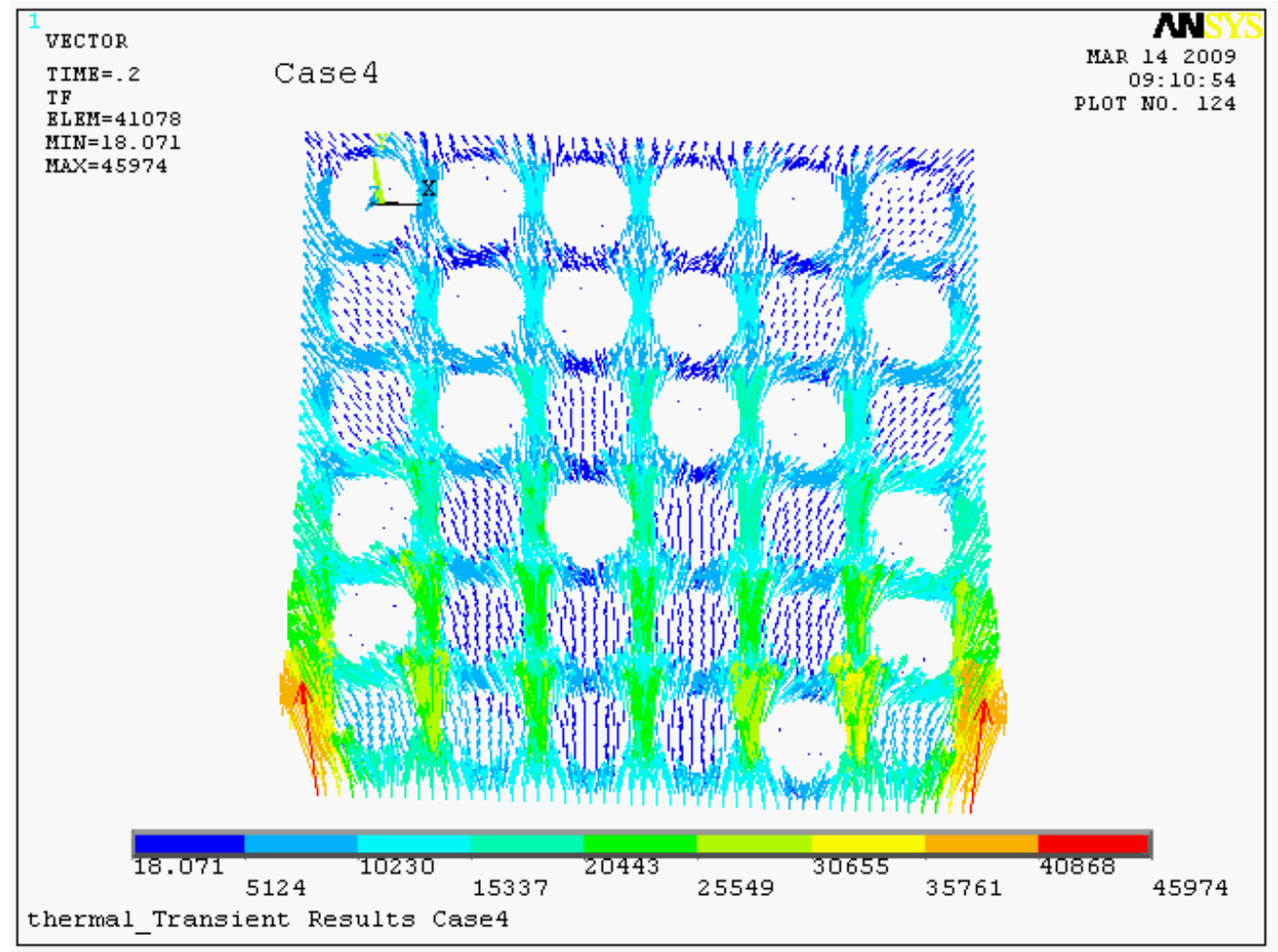

Figure 6.16: Distribution of heat flux over fiber with $0.25 \mathrm{VF}$ aerogel 


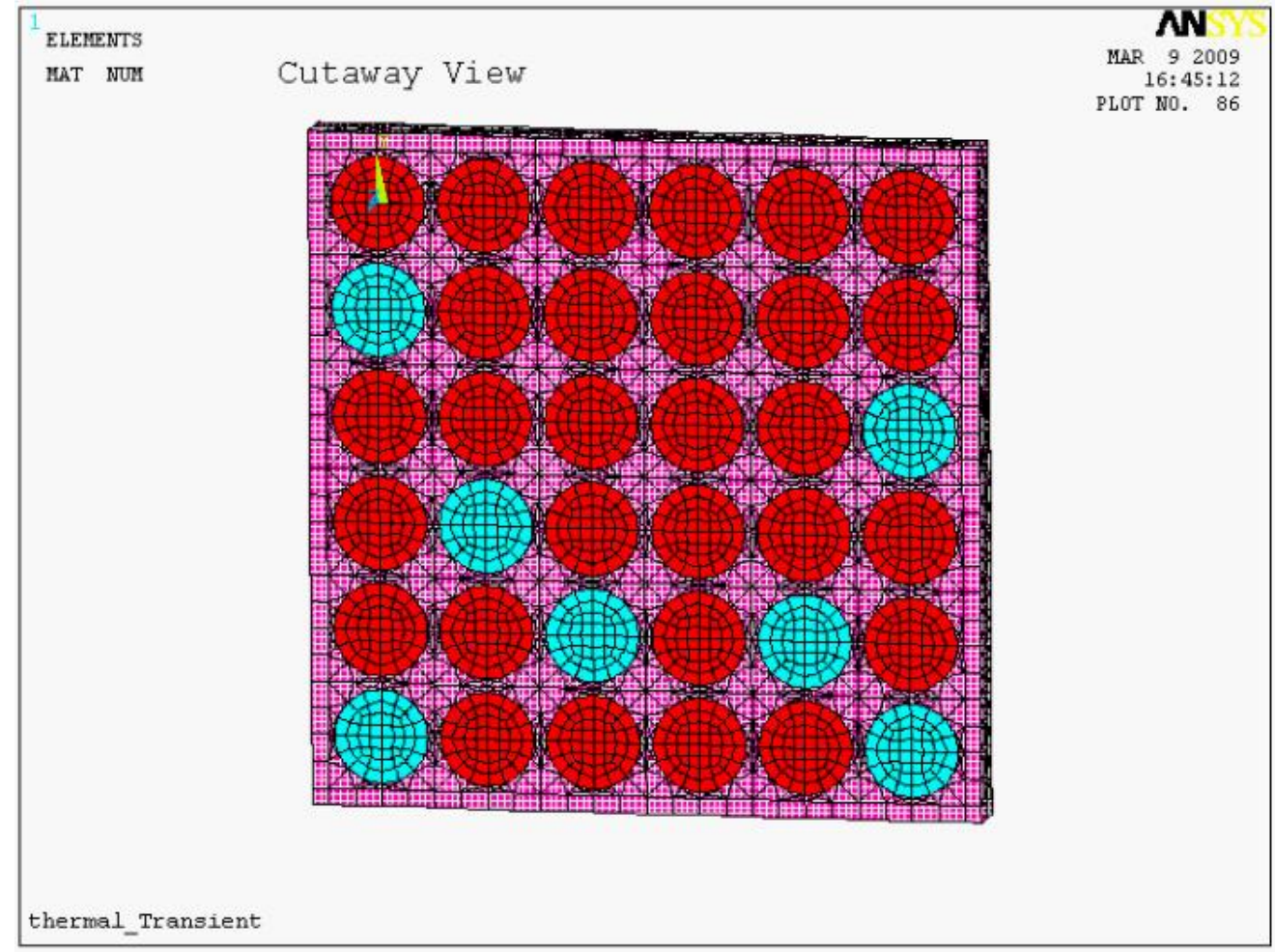

(a)

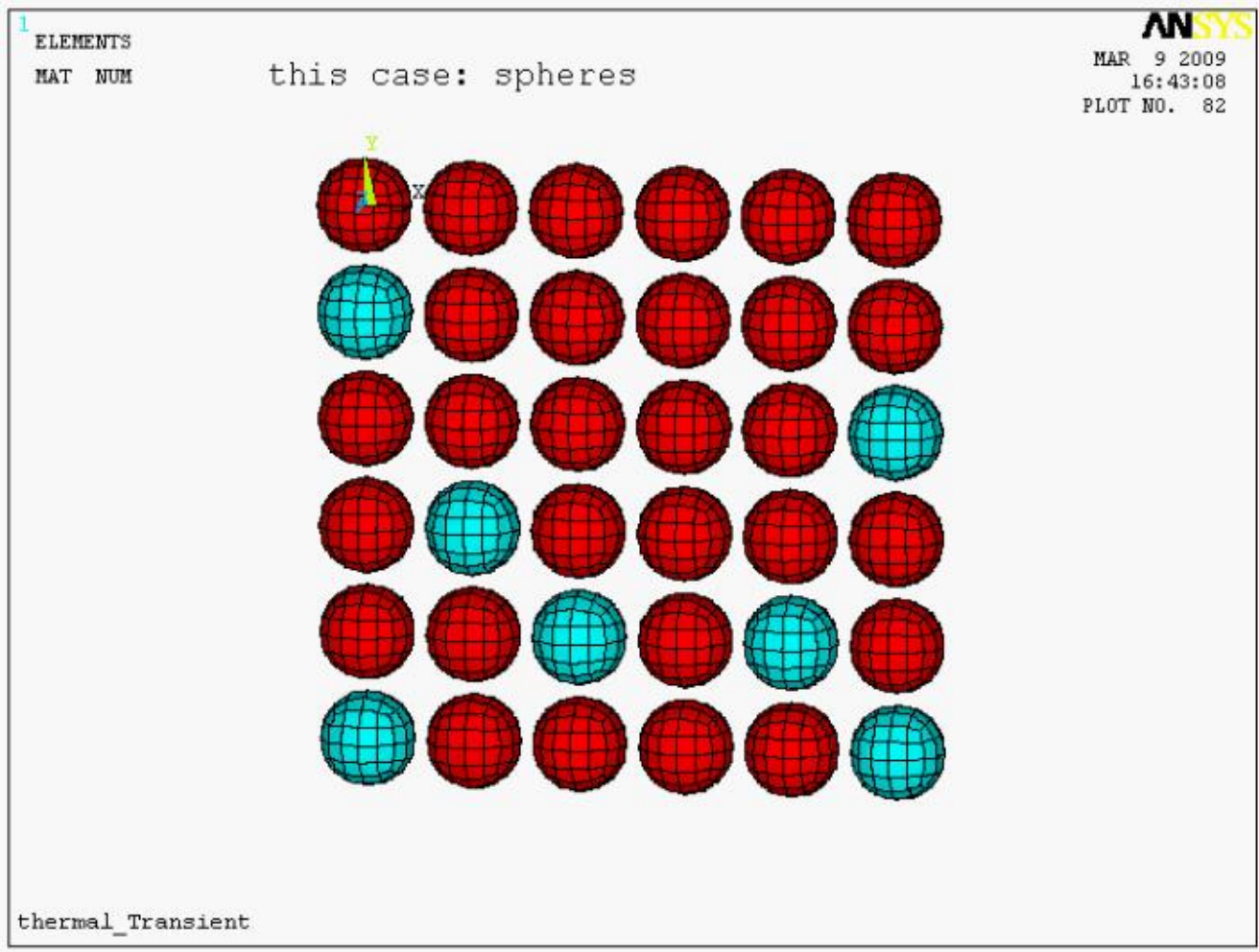

(b)

Figure 6.17: FEM mesh of: (a) fiber with $0.4 \mathrm{VF}$ aerogel and (b) aerogel particles 


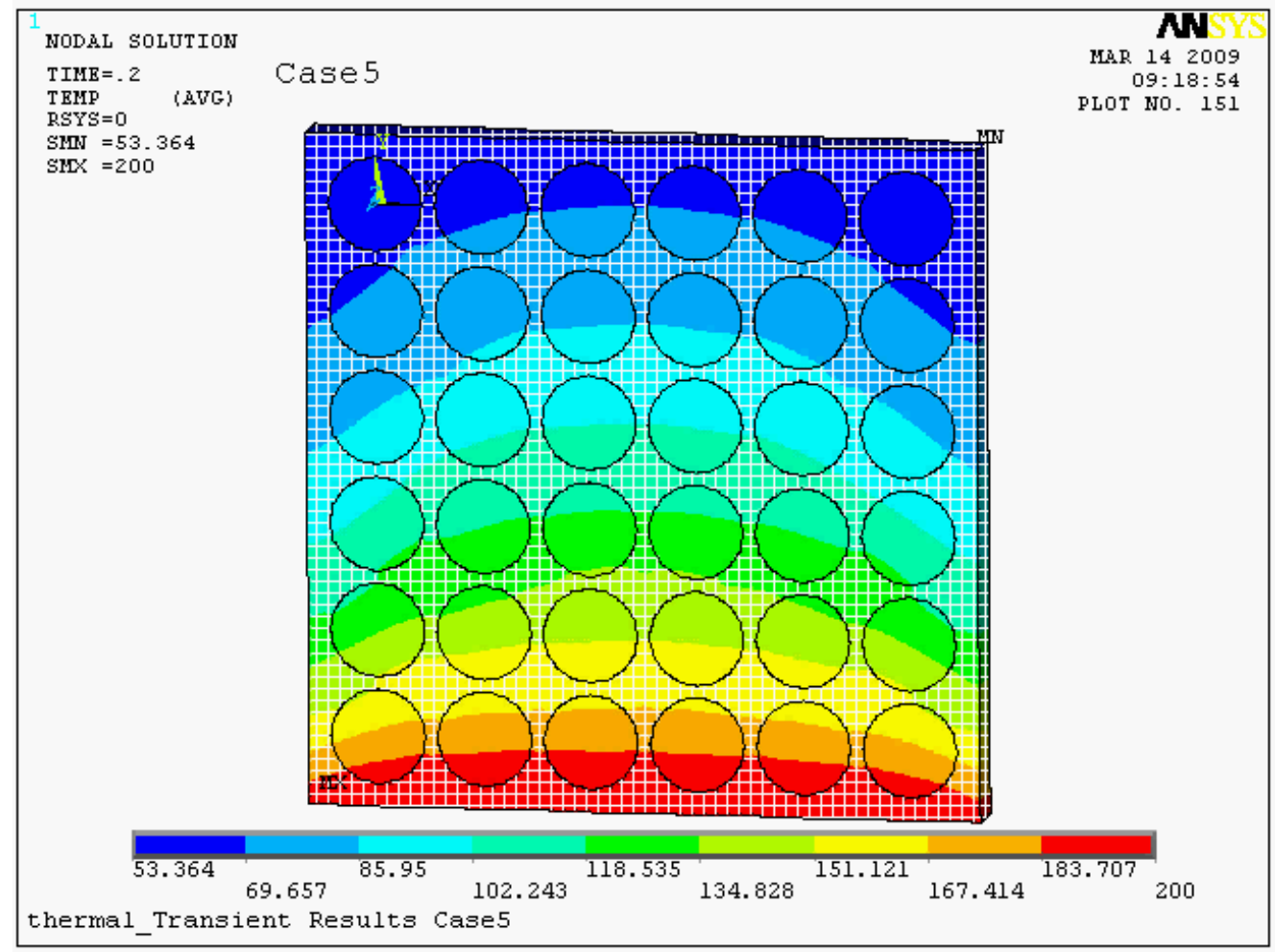

Figure 6.18: Distribution of temperature over fiber with $0.4 \mathrm{VF}$ aerogel

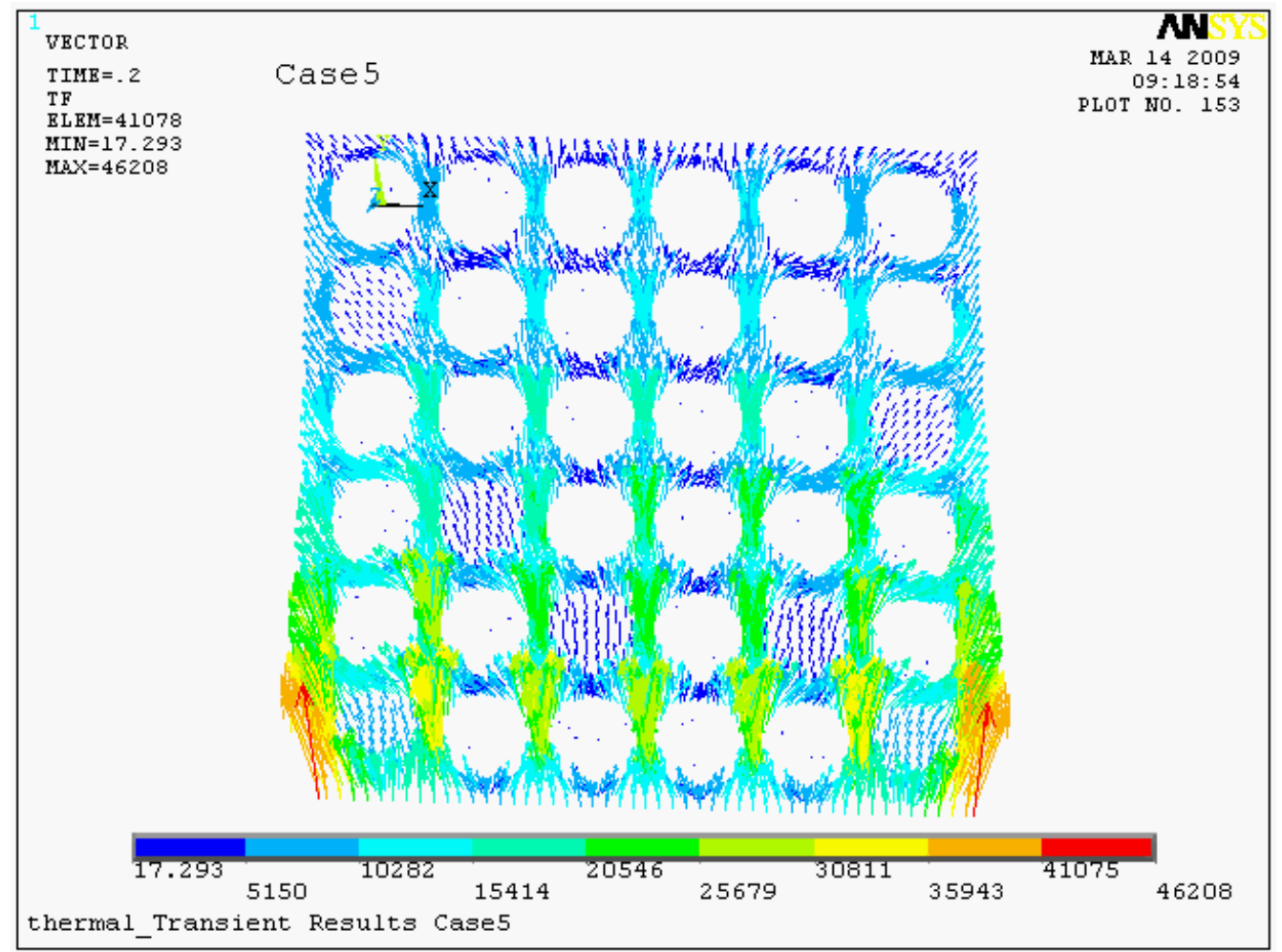

Figure 6.19: Distribution of heat flux over fiber with $0.4 \mathrm{VF}$ aerogel 
For each case of this computer modeling, a value of effective thermal conductivity was calculated along the y direction. This value was obtained by averaging nodal heat flux values and it's given by:

$$
q=\frac{Q}{A}
$$

The effective thermal conductivity is calculated then from:

$$
k_{y}=q \cdot\left(\frac{\Delta_{y}}{\Delta T}\right)
$$

One of the most important results of this finite element study is the variation of the thermal conductivity with the volume fraction of aerogel particles shown in figure 6.20 .

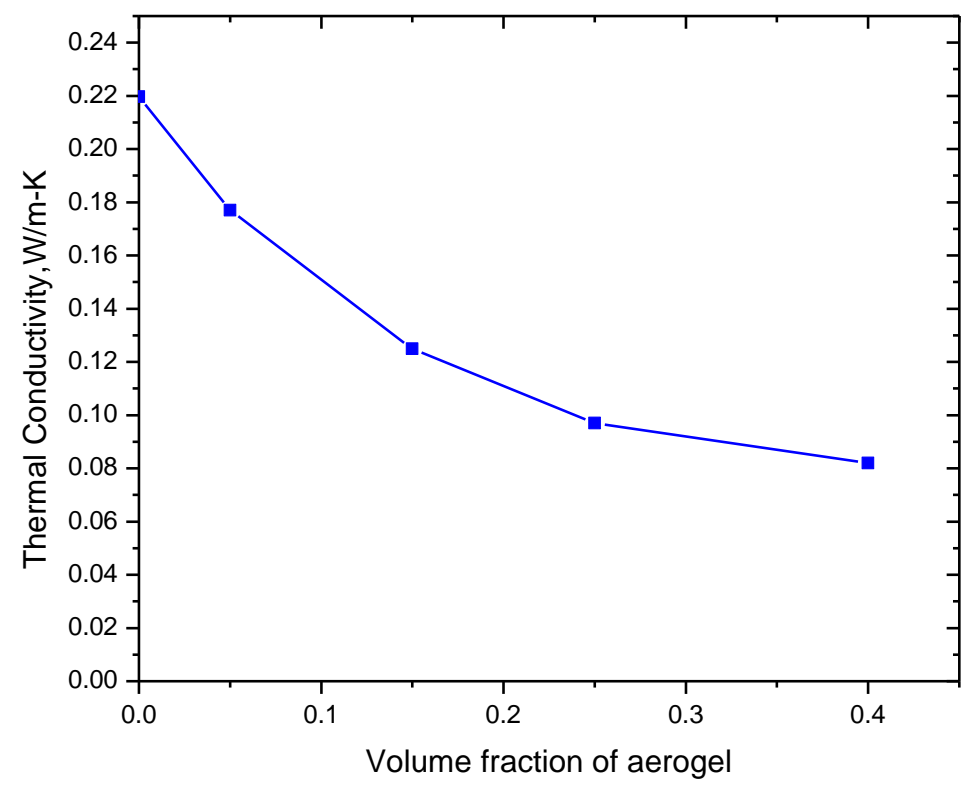

Figure 6.20: Variation of thermal conductivity with VF of aerogel 
FEA was performed to evaluate the cumulative effects of the mechanical degradation of the insulation blanket on their thermal transport properties and to investigate the influence of aerogel materials and their volume fraction on unsteady state heat transfer through the flexible blanket insulation model.

The visualization of the thermal and the heat flux distributions obtained by the FE modeling simulations during the heating process of the flexible blanket as shown in figures 6.9-6.20 give a better understanding of the status of structural and thermal temperature dependence. For all cases, the insulating blanket temperature decreases from its reference value at the bottom surface to a lower value at the top surface. However, the cooling rate is different for each case, increasing with aerogel volume fraction. The cooling rate, as a processing parameter, is implicitly defined using the temperature difference between the bottom and the top surfaces of the flexible aerogel blanket parameter. For case $1, \Delta_{T} \approx 142.8^{\circ} \mathrm{C}$ and increases up to $3.75 \%$ for case 5 . It can be seen that as the aerogel volume fraction increases, the temperature difference between the bottom and the top surfaces of the sample also increases and the heat flux decreases, which demonstrate the exceptional insulating properties of aerogel materials. Aerogel is a better insulator than air. This FEA also provides the capability for the modeling and prediction of the thermal conductivity of the flexible aerogel blankets. By comparing the thermal performance in the five cases, it is clear that the sample with more aerogel particles (case 5) offered superior insulating properties. The obvious basis for this advantage is the lower thermal conductivity of aerogel than air. These results clearly show the insulation effect of aerogel particles in the flexible blankets.

Figure 6.20 presents the variation of effective thermal conductivity of the flexible 
aerogel blanket with the volume fraction of aerogel. High volume fraction of aerogel clearly has better insulation performance than low volume fraction, as would be expected since the thermal conductivity of aerogel materials are less than air and fiber. As the fraction of low thermal conductivity material in the FE model increases, so does the insulating capability. At low volume fraction, where the aerogel particles occupy a very small portion of the flexible blanket, it offers worse insulation.

\section{9 Comparison of Numerical and Theoretical Models}

In figure 6.21, the effective thermal conductivity of the flexible aerogel blanket obtained from the FEM model is compared with the more accurate theoretical models obtained in this study.

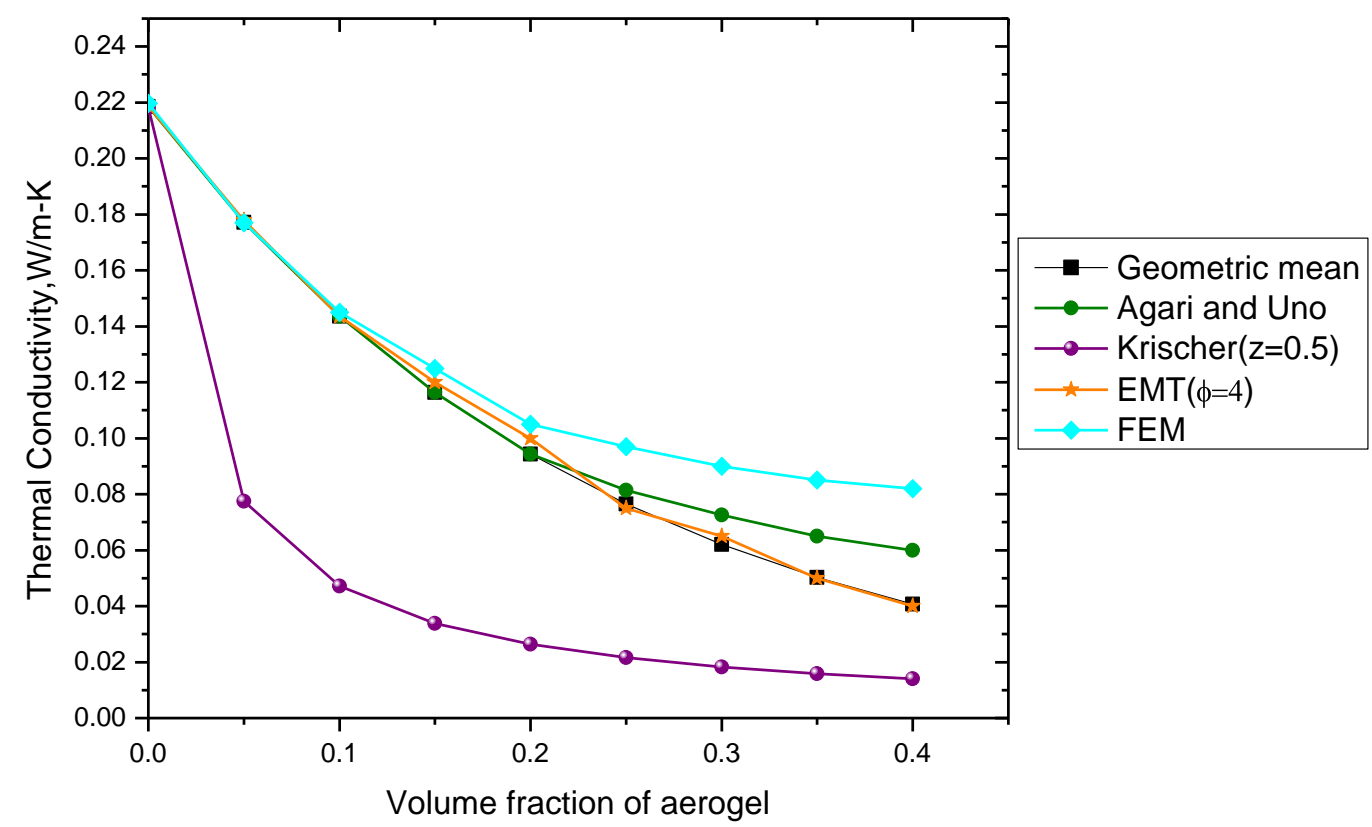

Figure 6.21: Comparison of the FEM and theoretical results

As can be seen from this figure, the effective thermal conductivity values of the flexible 
aerogel blankets obtained from the theoretical models were found to be significantly lower than the numerical model values. For $V_{\text {aerogel }} \leq 0.1$, the numerical and the theoretical models, except the Krischer model, perfectly predicted the same value for effective thermal conductivity. As the volume fraction of aerogel increased, the differences between the theoretical and the numerical values of thermal conductivity become much greater ranging from 0.01 to $0.05 \mathrm{w} / \mathrm{m}-\mathrm{K}$. EMT, Geometric mean and Agari and Uno and FEM models predicted thermal conductivity values fairly accurately up to $10 \%$ of aerogel particles content, whereas beyond $10 \%$ of aerogel particles content, all models predicted different thermal conductivity values. It was suggested that the discrepancy was due to the uniformity in the shape of aerogel and air pocket particles, which increased the values for the effective thermal conductivity obtained from the FEM model. In contrast to other theoretical models, the Krischer model, widely used in food engineering, was not able to provide a good overall agreement to the FEM results. 


\section{Chapter 7 Conclusion}

The response of flexible aerogel blankets to tensile and three-point bending loads was examined. Analysis of stress-strain curves for tensile tests, load displacement curves for the three point bending tests and correlations with scanning electron microphotographs revealed the failure and deformation mechanisms of the insulation blankets.

Before degradation, the surface of the flexible aerogel blankets was smooth, and became rough and took on a wavy appearance after degradation. From the microscopy images of an unloaded specimen, the network structure of the aerogel was intact, but a cavity was present after displacement. Morphological changes seen in three point bending loaded specimens were similar to those in tensile loaded ones, but more marked in each subsequent test. At the final stage of the mechanical tests, almost all of the network structure of aerogel had been completely destroyed. The degradation behaviors of all the insulation blankets were similar.

Under the action of tensile loads, aerogel composites were extended and the dimensions of the cracks became larger. Failure began with a peak retentive value followed by a rapid decrease in stress. The mechanical measurements under tensile loads showed that Pyrogel materials AR 5400 and AR 5403 X decayed at a much slower rate than Spaceloft materials AR 3103 and AR 3103X, due mainly to their physical nature and chemical composition. Pyrogel materials AR 5400 and AR 5403 X also had approximately four and five times more tensile strength than Pyrogel materials AR 5223 and AR 5401 respectively at a strain of $0.20 \mathrm{~mm} / \mathrm{mm}$. As expected, Pyrogel materials AR 5400 and AR 5403 X had the highest stiffness based on the value of their modulus. 
Understanding the mechanics of three-point bending was crucial for evaluating the flexural strength of flexible aerogel materials. Spaceloft composites in three- point bending tests had significantly lower flexural load failure than the Pyrogel ones. A comparison between the blankets specimens yields a reduction of $86 \%$ in their moment capacity and a reduction of $50 \%$ in their stiffness. Under the three point bending tests, all blanket samples recovered to their original thickness. Flexible aerogel blankets under three point bending loads degraded slower than that under tensile loads.

During the mechanical testing of the flexible aerogel blankets, their weight loss continuously increased. When the cyclic bending test was performed, the variation in weight loss percentage increased with each cycle, displacement and thickness of the sample. Although Pyrogel samples AR 5401X and AR 5401 had less flexural strength than Pyrogel AR 5400, AR 5223 and AR 5403X, their weight loss was small because of their thinness. In Pyrogel and Spaceloft specimens of the same thickness, the weight loss was proportional to their flexural strength. In a more explicit way, Spaceloft AR 3103 had three time less flexural strength than Pyrogel AR 5400 and consequently has approximately 7\% more weight loss than Pyrogel AR 5400. Aerogel composites with coated surfaces underwent less weight loss than those not coated. Pyrogel specimen AR 5401X had 1\% less weight loss than Pyrogel specimen AR 5401 and Spaceloft materials AR 3103X had 2\% less mass loss than AR 3103. The use of a metalloid adhesive (silicon) on the surface of the insulation blankets significantly increased the bending failure and decreased the mass loss.

During the hot-cold measurements, both Pyrogel and Spaceloft materials showed higher thermal performance at higher temperatures. Spaceloft materials reached their 
steady state faster which means they had higher thermal diffusvity and thermal stability.

The mechanical properties of flexible aerogel blankets can be improved significantly by coating the surface of these insulating blankets, but this reduces their thermal properties. The relative temperature drop increased as the hot plate temperature increases. In the project, a correlation between the thermal and the mechanical studies of flexible aerogel blankets was presented. It showed how that the changes in the microstructure brought about by mechanical degradation significantly affected the thermal performance of flexible aerogel blankets. To determine the effects of tensile and three-point bending loads on the thermal performance of flexible aerogel blankets, the temperature difference between the bottom and the top surface of the blankets was needed as a function of displacement. Experiments were therefore developed and carried out to measure these properties up to high displacements.

The three-point bending loads and the tensile loads up to breaking were found to have very different effects on the thermal properties of the studied Pyrogel and Spaceloft blanket materials. Higher loads led to lower temperature differences between the bottom and the top surface of all the flexible aerogel blanket specimens. The temperature difference between the bottom and the top surface of the flexible aerogel blankets decreased with the increase in cycles and tensile displacements until their minimum value was reached. For all the flexible aerogel specimens, the temperature difference between the bottom and the top surfaces showed a similar trend in line with the number of cycles and displacements. These results displayed by the flexible aerogel materials were attributed to the thermal softening of the materials that preceded the production and the propagation of cracks leading to ultimate failure. The temperature 
differences displayed by the specimen stabilized when the plastics deformation (in threepoint bending) and the fracture (tensile) occurred. Pyrogel AR5400 and AR5403 X presented superior thermal performance due to a much smaller temperature difference changes under mechanical loads. Thus, Pyrogel AR5400 and AR5403 X materials are useful for higher thermal resistance applications.

The theoretical and numerical heat transfer models provided the ability to study the heat transfer effect in flexible aerogel blankets from their structural characteristics, including the blanket structure, the heat flow orientation, the volume fraction and the thermal properties of each of their constituents, i.e, fiber, aerogel and air. The theoretical model results predicted an estimation of the effective thermal conductivity of the flexible aerogel blanket in a $40 \%$ range of the aerogel content.

The numerical model results gave a better understanding of the heat transfer process that over the surfaces of the insulation blanket. This model was developed to predict the temperature distribution in a composite exposed to heat flux. Flexible aerogel blankets with higher aerogel volume fractions exhibited higher heat dissipation resulting in a faster cooling rate. Aerogel materials formed an excellent thermal barrier. The FE model results revealed that thermal conductivity decreased with aerogel content in the composite material.

The results of the FE modeling of the effective thermal conductivity obtained compared fairly well with the theoretical results. These models showed very interesting predictions concerning the effects of air-pocket and aerogel particles volume fraction. For low aerogel particles content (less than 10\%), the results of the theoretical and the numerical models showed good agreement but discrepancies were observed at high 
aerogel particles content. Such a fundamental approach to studying heat transfer issues in flexible aerogel blankets has numerous practical applications, including the optimization of the flexible aerogel blanket configurations and their processing methods in order to keep their insulating and structural capacities. 


\section{Chapter 8 Recommendations for Future Work}

This study showed that when degradation process of the flexible aerogel blanket occured, the fiber deterioration was neglected. This study assumed perfectly bonded aerogel particles/fiber interfaces. Further research could study the effect of fiber cracking in combination with interfacial debonding on the effective thermal conductivity of flexible aerogel blankets and investigate configurations with random aerogel and air particles distribution. More aerogel particles could be included in the FE model, e.g., using an $\mathrm{m} X \mathrm{n}$ model with $\mathrm{m}>6$ and $\mathrm{n}>6$. These particles could also be arranged in a different pattern, e.g., in the form of hexagon. Studies of these cases are readily achievable with the FE model and will be left for the future. The FE model is recommended to be simulated for cases which involve partial or complete fracture of aerogel particles. The presence of some opacifying agents like carbon black inside the flexible aerogel blanket may introduced the influence of radiative heat transfer mode determined by the radiative heat exchange between the surfaces of fiber and aerogel or air particles, which was neglected in this analysis.

In the course of this research project, additional thermo-mechanical characterizations were identified as targets for further research. These characterizations include Dynamic Mechanical Analysis (DMA) and Thermo Mechanical Analysis (TMA). They supply valuable information about structural phase transitions that a material can undergo. The Perkin Elmer Dynamic Mechanical Analyzer DMA7e can be used to run the DMA and TMA of flexible aerogel blanket experiments. The DMA analysis can be employed in either a three-point bend mode or a tensile mode by heating the samples from $-20^{\circ} \mathrm{C}$ to $260^{\circ} \mathrm{C}$ at a frequency of $1 \mathrm{~Hz}$, a strain level of $0.1 \%$ and a heating rate of 
$2^{\circ} \mathrm{C} / \mathrm{min}$. In DMA, a complex modulus, an elastic modulus and an imaginary modulus can be obtained as a function of temperature each time a sine wave is applied to the sample, allowing us to sweep across a temperature or frequency range.

So, if these different moduli were found, the ability of flexible aerogel blankets to either store or lose energy could be examined and a better characterization of the material can be set out. TMA yields information about mechanical properties by applying a static load to a sample under varying temperatures and then obtains the dimensional changes. One advantage of TMA is the measurement given of the thermal expansion and the glass transition temperature of the insulation blanket. 


\section{REFERENCES}

1. Hotaling, S., "Ultra-Low Density Aerogel Optical Applications," Journal of Material Research, Vol. 8, pp 352-355, 1993.

2. "Guinness Records Names JPL's Aerogel World's Lightest Solid," http://stardust.jpl.nasa.gov/news/news93.html , NASA, 2003.

3. Baumann, T., et al., "High Surface Carbon Aerogel Monoliths with Hierarchical Porosity," Journal of Non-Crystalline Solids, Vol.354, pp.3513-3515, 2008.

4. Lerner, E., "Less is More with Aerogels," AIP Vol.10, p26, 2004.

5. Hrubesh, L., and Poco, J., "Thin Aerogel Film for Optical, Thermal, Acoustic and Electronics Applications," Journal of Non-Crystalline Solids, Vol.188, pp 46-53, 1995.

6. Kim, G., and Hyun, S., "Effect of Mixing Thermal and Mechanical Properties of Aerogel-PVB Composites," Journal of Materials Science, Vol.38, pp1961-1966, 2003.

7. "Aerogels-Aerogel Materials-Nanofoams, MarkeTech International," http://www.mkt-intl.com/aerogels/index.html , MarkeTech International, 1996.

8. Kistler, S., "Coherent Expanded Aerogels and Jellies,” Nature No 3211, Vol.127, Stockton, and Stanford University, California, 1931.

9. Kistler, S., “Coherent Expanded Aerogels," Journal of Physical Chemistry, Vol.136, pp52-64, 1932

10. Kistler, S., "Aerogels of Silica, aluminum, chromium and etc.," U.S. Patent 2,093,454., 1937.

11. Kistler, S., “Aerogels,” U.S. Patent 2,249,767, 1941.

12. Teichner, S. et al., "Inorganic Oxide Aerogel," Advances in Colloid and Interface Science, Vol. 5, pp 245-273, 1976.

13. Nicolaon, G., and Teichner, S., "Method of Preparing Inorganic Aerogels" US patent No 3,672,833, 1972.

14. Poelz, G. and Riethmueller, R., "Preparation of Silica Aerogel for Cherenkov Counters," Nuclear Instrumental Method, Vol.195, p 491, 1982. 
15. Adachi, I., et al. "Study of threshold Cherenkov Counter Based on Silica Aerogels with Low-Refractive Indices," Nuclear Instruments and Methods in Physical Review A, Vol. 355, pp 390-398, 1995.

16. Denniel, S. and Blair, C., "Aerogel Insulation for Deepwater Reelable Pipe-in-Pipe", Offshore Technology Conference 16505, Houston, Texas, 2004.

17. Fricke, J., “Aerogels,” Proceedings in Physics, vol.6, Springer-Verlag, Berlin, 1986.

18. Tewari, P., Lofftus, K. and Hunt, A., "Structure and Chemistry of Sol-Gel Derived Transparent Silica," International Conference on Ultrastructure Processing of Ceramics, Glasses and Composites, pp.17, LLNL, California, 1985.

19. Hunt, A., "Light-Scattering Studies of Silica Aerogels," International Conference on Ultrastructure Processing of Ceramics, Glasses and Composites, pp.15, LLNL, California, 1983.

20. Ayers, M., and Hunt, A., "Molecular Oxygen Sensor Based On Photoluminescent Silica Aerogel," Journal of Non-Crystalline Solids, Vol. 225, pp.343-347, 1998.

21. Zeng, J., Stevens, P., Hunt, A. and Lee, D. "Thin-Film-Heater Thermal Conductivity Apparatus and Measurement of Thermal Conductivity of Silica Aerogel," International Journal of heat and Mass Transfer, Vol.39, pp2311-2317, 1996.

22. Hrubesh, L., "Development of Low Density Silica Aerogel as a Capture Medium for High-Velocity Particles," NASA Technical Paper, No.185930, 1989.

23. "Aerogel: Catching comet Dust," http://stardust.jpl.nasa.gov/tech/aerogel.html, NASA, 2005.

24. "Cryogel”, http://www.aerogel.com/products/pdf/Cryogel.html, Aspen Aerogel, 2006.

25. “Aerogel Batting with Fibrous Batting,” United States Patent No 7078359, 2000.

26. Ristick-Lehman, C. et al., "Aerogel/PTFE composite insulating materials," United States Patent No 7226969, 2007.

27. Ryu, J. et al., "Flexible Aerogel Superinsulation and its manufacture," United States Patent No 6068882, 2000.

28. "Lightweight Multifunctionality for Demanding Environments," http://www.aerogel.com/markets/military.html, ASPEN Aerogels, 2006.

29. Brinker, C., and Sherer, G., Sol-Gel Science, Academic Press, San Diego, 1990. 
30. Husing, N. and Schubert, U., Synthesis of Inorganic Materials," Wiley-VCH, Weinheim, 2004.

31. Smith, D., Stein, D., Anderson, J. and Ackerman, W., "Sintering Aerogels," Journal of Non-Crystalline Solids, Vol.186, p104, 1995.

32. Iler, R., The Chemistry of Silica, Wiley, 1979

33. Husing, N., and Schubert, U., "Aerogels-Airy Materials: Chemistry, Structures and Properties," Journal of Applied Chemistry, Vol. 37, pp 22-45, 1998.

34. Hiemenz, P., and Timothy, L., Polymer Chemistry, CRC Press, 2007.

35. Dutoit, D., Schneider, M., Fabrizioli, P., and Baiker, A., "Vanadia- Silica Low Temperature Aerogels: Influence of Aging and Vanadia Loading on Structural and Chemical properties," Chemistry of Materials, Vol. 8, pp 734-743, 1996.

36. Haereid, S., Nilsen, V., Ranum, V. and Einarsrud, A., “Thermal and Temporal Aging of Two Step Acid-Base Catalyzed Silica Gels in Water/Ethanol Solutions," Journal of Sol-Gel Science and Technology, Vol. 8, pp 153-157, 1997.

37. Wright, J. and Sommerdijk, N., Sol-Gel Materials Chemistry and Applications, CRC Press, 2000.

38. Perrut, M. and Francais, E., "Aerogel Drying," State of the art book on Supercritical Fluids, pp 129-134, 2004

39. Sherer, G., Hdach, H.and Phalipou, and J. "Thermal Expansion of Gels: A novel Method for Measuring Permeability,"Journal of Non-Crystalline Solids, Vol. 130, pp. 157, 1991.

40. Gauthier, B., Bakrania, S., Anderson, A. and Caroll, M., "A Fast Supercritical Extraction Technique for Aerogel Fabrication," Journal of Non-Crystalline Solids, Vol. 350, pp 238-243, 2004.

41. Ramamurthi, S. et al., “Aerogel matrix Composite," United States Patent No 5306555, 1993.

42. Hrubesh, L., Pekala, R. "Thermal Properties of Organic and Inorganic Aerogels," Journal of Materials Research, Vol. 9, pp 731-738, 1994.

43. Bradley, D., et al., Alkoxo and Aryloxo Derivatives of Metals , Academic Press, San Diego, 2001. 
44. Buckley, M., and Greenblatt, M., "The Preparation and Characterization of Silica Aerogels and Xerogels doped With Transition Metals Species," Journal of NonCrystalline Solids, Vol.146, pp97-110, 1992.

45. "Coated insulation articles and their manufacturing," United States Patent No 7,078,359 B2, 2006.

46. Chandradass, J., Kang, S., and Bae, D., "Synthesis of Silica Aerogel Blanket By Ambient Drying Method Using Water Glass Based Precursor and Glass Wool Modified by Alumina Sol," Journal of Non-Crystalline Solids, Vol. 354, pp.41154119, 2008.

47. Cross, J., and Fricke, J., "Ultrasonic Velocity Measurements in Silica, Carbon and Organic Aerogels.”Journal of Non-Crystalline Solids, Vol. 145, p 217, 1992

48. Gronauer, M., Kadur, A. and Fricke, J., in J. Fricke (Ed) Aerogels, Springer, Berlin, 1986.

49. Woignier, T., Phalippou, J., Hdach, H., and L.S.M.V., "Mechanical Properties of Silica Alcogels and Aerogels," MRS symposium Proceedings Vol.180, pp. 10871098, 1990

50. Arvidson, J. and Scull, L., "Compressive Properties of Silica Aerogels at 295, 76, and 20 K," Advanced Cryogenic Engineering Materials, Vol. 32, p 243, 1986

51. LeMay, J., Tillotson, T., Hrubesh, L. and Pekala, R., "Microstructural dependence of Aerogel Mechanical Properties" MRS symposium Proceedings 80, p 321, 1990.

52. Pekala, R., Hrubesh, L., Tillotson, T., LeMay, J., “Aerogel Manufacture, Structure, Properties and Applications," MRS symposium Proceedings 207, p 197, 1991.

53. Parmenter, K., and Milstein, F., "Mechanical Properties of Silica Aerogels," Journal of Non-Crystalline Solids, Vol.223, pp179-189, 1998

54. Moner-Girona, M., Martinez, E., Roig, A., Esteve, J., and Molins, E., "Mechanical Properties of Silica Aerogels Measured by Microindentation: Influence of Sol-Gel Processing Parameters and Carbon Addition," Journal of Non-Crystalline Solids, Vol.285, pp. 244-250, 2001.

55. Cross, J., Fricke, J., Pekala, R., and Hrubesh, L., "Elastic Nonlinearity of Aerogels," Physical Review B, Vol.45 No 22, p 12774, 1992.

56. Woignier, T. et al. "Plasticity in Aerogels," Journal of Sol-Gel Science and Technology, Vol. 8, pp. 789-794, 1997. 
57. Scherer, G., Smith, D., Qiu, X., and Anderson, J., "Compression of Aerogels," Journal of Non-Crystalline Solids, Vol. 186, pp 316-320, 1995.

58. Etienne, P., Woignier, T., Alaoui, A. and Phalippou, J. "Brittle Fracture of Aerogels: Stress Corrosion in Alcoholic Environment," Journal of Sol-Gel Science and Technology, Vol. 8, pp. 801-806, 1997.

59. Woignier, T., Phalippou, J., and Vacher, R., "Parameters Affecting Elastic Properties of Silica Aerogels," Journal of material research, Vol.4, pp688-692, 1989.

60. Bardy, E., Mollendorf, J., and Pendergast, D. "Thermal Conductivity and Compressive Strain of Aerogel Insulation Blankets under Applied Hydrostatic Pressure," Journal of Heat and Mass Transfer, Vol. 129, pp 232-236, 2007.

61. Incropera, F., and De Witt, D., Fundamentals of Heat and Mass Transfer, John Wiley and Sons, Third Edition, pp.100-103, 1990.

62. Rettelbach, Th., Sauberlich, J., Korcher S. and Fricke, J. "Thermal Conductivity of Silica Aerogel Powders at Temperatures from 10 to 275 K," Journal of NonCrystalline Solids, Vol. 186, pp 278-284, 2005.

63. Lu, X., Wang, P., Arduini-Schuster, M., Kuhn, J., Buttner, D., Heinemann, U and Fricke, J "Thermal Transport in Organic and Opacified Silica Monolithic Aerogels," Journal of Non-Crystalline Solids, Vol. 145, pp.207-210, 1992.

64. Ritcher, K., Norris, P., and Tien, C. "Aerogels: Applications, Structure, and Heat Transfer Phenomena," Annual Review of heat transfer, Vol 6, pp62-113, 1996.

65. Hummer, E., Lu, X., Rettelbach, Th., and Fricke, J "Heat Transfer in Opacified Aerogel Powders," Journal of Non-Crystalline Solids, Vol. 145, pp.211-216, 1992.

66. Rettelbach, Th., et al., "Thermal Conductivity of IR-Opacified Silica Aerogel Powders Between 10K and 250 K," Journal of Applied Physics, Vol. 28, pp.581$587,1995$.

67. Chandler, E., Calef, D., and Ladd, A., "Computer Modeling of Organic Aerogels." Final Report of 93-SR-062, LLNL, 1994

68. Gross, J. et al., "Ultrasonic Evaluation of Elastic Properties of Silica Aerogels," Modeling and Simulations in Materials Science and Engineering, Vol.168, p.235, 1993

69. Ma, H., Prevost, J., Jullien, R., and Scherer, W., "Elasticity of DLCA Model Gels With Loops," International Journal of Solids and Structures, Vol. 39, pp.4605-4614, 2002 
70. Ma, H., Prevost, J., Jullien, R., and Scherer, W., "Mechanical Structure-Property Relationships of Aerogels," Journal of Non-Crystalline Solids, Vol. 277, p 127, 2000.

71. Gibson, L., and Ashby, M., Cellular Solids: Structures and Properties, Cambridge University Press, UK, 1997.

72. Ma, H., Prevost, J., Jullien, R., and Scherer, W., "Computer Simulation of Mechanical Structure-Property Relationships of Aerogels," Journal of NonCrystalline Solids, Vol. 285, pp.216-221, 2001.

73. Paul, H. and Diller, K., "Comparison of Thermal Insulation Performance of Fibrous Materials for the Advanced Space Suit," Journal of Bioengineering, Vol.125, pp 639647, 2003

74. "Spaceloft 3251, 6251, 9251 Flexible Insulation for Industrial, Commercial and Residential Applications," http://www.aerogel.com/products/pdf/Spaceloft_3251_6251_9251_DS.pdf, ASPEN Aerogels, 2007.

75. "Pyrogel 3350,6350,10350 Flexible Insulation for High Temperatures," http://www.aerogel.com/products/pdf/Pyrogel_3350_6350_10350_DS.pdf, ASPEN Aerogels, 2007.

76. Aspen aerogels, "Description of Aerogel and Market Applications," Company Brochure, 2008.

77. Aspen aerogels, "Nanotechnology at Work in Offshore Oil, LNG, and Oil Sands," Company Litterature, 2008.

78. Butterfield, A., and Wilk, R. "Design and Performance of Advanced Window Systems," ASME Proceedings of IMECE No 81363, pp 541-546, Orlando, Florida, 2005.

79. “Window Industry Technology Roadmap," www.Eren.doe.gov/buildings, United States Department of Energy, 2000.

80. Aspen aerogels, “Thin Spaceloft Insulation,” Company Literature, 2008.

81. Aspen aerogels, “Spaceloft Insul-Cap,” Company Literature, 2008.

82. "Aerogel Insulation Reduces Heat Loss on Small Pipes in Confined Spaces," http://www.aerogel.com/markets/c_hotwaterpipes.html, ASPEN Aerogels, 2006.

83. Aspen aerogels, "Extreme Insulation for Extreme Environments," Company Literature, 2008. 
84. Weaver, D. and Duke, M., "Mars Exploration Strategies: A reference Program and Comparison of Alternative Architectures," AIAA Transactions, 93-4212, pp.5-6, 1993.

85. "Aerogel Insulation Installs Faster and Saves Costs on Large Vessel," http://www.aerogel.com/markets/c_vessel.html, ASPEN Aerogel, 2006.

86. Dunlap, M., and Adaskaveg, J., "Introduction to the Scanning Electron Microscope," Facility For Advanced Instrumentation, University of California at Davis, 1997.

87. Reimer, L., Scanning Electron Microscopy, Springer-Verlag, Berlin, 1985.

88. Gareth, T., and Fulrath, R., Electron Microscopy and Structure of Materials, University of California Press, 1972.

89. Goldstein, J., et al., Scanning Electron Microscopy and X-ray Microanalysis, Plenum Press, New York, 1981.

90. Pajonk, G., et al., "Microstructural Characterizations of silica Aerogels using SEM," Journal of Material Science, Vol. 31, pp. 5683-5689, 1996.

91. Piggott, M. “A Three Point Bend Tester for Stronger Fibers,” Journal of Physics E: Scientific Instruments, Vol.1, pp. 444-446, 1968.

92. ASTM D 7264 - 07 Standard Test Method for Flexural Properties of Polymer Matrix Composite Materials, 2007.

93. Sandor, B., Fundamentals of Cyclic Stress and Strain, University of Wisconsin Press, 1972.

94. ASTM D 3039 - 00 Standard Test Method for Tensile Properties of Polymer Matrix Composite Materials, 2000.

95. Obaid, A., Anderson, S., Gillepsie, Jr., Vaidyanathan, R. and Studley, A., "Investigation of Thermal and Acoustic Performance of Aerogel-Based Glass Fiber Composites," Proceedings $50^{\text {th }}$ International SAMPE symposium and Exhibition, Vol.50, Long Beach, California, 2005.

96. Venkateswara, A. et al. "Synthesis and Characterization of Hydrophobic Silica aerogels Using Trimethylethoxysilane as a Co- Precursor," Journal of Sol-Gel Science and Technology, Vol. 27, pp.103-109, 2003.

97. Stalder, A.F. et al., "A Snake-Based Approach to Accurate Determination of Both Contact Points and Contact Angles," Colloids and Surfaces A: Physicochemical And Engineering Aspects, Vol. 286, no 1-3, pp. 92-103, 2006. 
98. Jones, R., Mechanics of Composite Materials, Taylor and Francis, Inc., 1999.

99. Rauch, H., Sutton, W., and Mccreight, L., Refractory Materials: Ceramic Fibers and Fibrous Composites, Vol. 3, Academic Press New York, 1968.

100. Rauch, H., Ceramics Fibers and Fibrous Composite Materials, Academic Press, New York, 1968.

101. Rosa, D., et al., "The Use of Optical Microscopy to Follow the Degradation of iPP Subjected to Natural and Accelerated Aging," Polymer Testing, Vol.24, pp.10221026, 2005.

102. Unger, D., Analytical Fracture Mechanics, Academic Press, New York, 1995.

103. Woignier, T. et al., "Evolutions of Mechanical properties During the Alcogel, Aerogel-Glass Process,” Journal of Non-Crystalline, Vol.147, pp. 672-680, 1992.

104. Etienne, P., Despetis, F., and Phalipou, J., "Subcritical Crack Velocity in Silica Aerogels," Journal of Non-Crystalline, Vol.225, pp. 266-271, 1998.

105. Alaoui, H. et al., "Stress Intensity Factor in Silica Alcogels and Aerogels," Journal of Non-Crystalline, Vol.265, pp. 29-35, 2000.

106. Despetis, F., Etienne, P and Etienne-Calas, S., "Subcritical Crack Growth in Silica Aerogel," Journal of Non-Crystalline Solids, Vol. 344, pp 22-25, 2004.

107. Liebowitz, R., Fracture: Microscopic and Macroscopic fracture, Vol.1, Academic Press, New York, 1968.

108. Gdoutos, E., et al., "Nonlinear Behavior of Composite Sandwich Beamsin ThreePoint Bending," Experimental Mechanics, Vol. 41, No2, pp.182-189, 2001.

109. Shatsov, A., "Mechanical Properties of Porous Materials," Journal of Metal Science and Heat Treatment, Vol. 45, pp8-11, 2003.

110. Rosen, B., "Tensile Failure of Fibrous Composite," AIAA Journal Vol. 2, pp19851911, 1964.

111. ASTM D30369 (1995) Standard Test Method for Tensile Properties of Polymer Matrix Composite Materials, 1995.

112. Yee, R.Y. and Stephens, T.S., "A TGA Technique for Determining Graphite Fiber Content in Epoxy Composites," Thermochimica Acta, Vol 272, pp.191-199, 1996. 
113. Chowdhury, A., Thompson, P. and Milne S., "TGA-FTIR Study of a Lead Zirconate Titanate Gel made from a Triol-Based Sol-Gel System," Thermochimica Acta Vol. 475, pp.59-64, 2008.

114. Liu, Y. et al.,"Thermal Stability of Epoxy-Silica Hybrid Materials by Thermogravimetric Analysis," Thermochimica Acta, Vol 412, pp.139-147, 2004.

115. Floury, J., Carson, J., and Pham, Q., "Modeling thermal conductivity in heterogeneous Media with the finite element method," Journal of Food Bioprocess Technology, Vol.1, pp161-170, 2008.

116. Maxwell, J., A Treatise on Electricity and Magnetism, $3^{\text {rd }}$ ed. New York, Dover, 1954.

117. Russell, H., "Principles of Heat Flow in Porous Insulation," Journal of American Ceramic Society, Vol.18, p1, 1935.

118. Kumlutas, D., Tavman, I., and Coban, M., "Thermal Conductivity of Particle Filled Polyethylene Composite Materials," Composite Science and Technology, Vol.63, pp.113-117. 2003.

119. Tsao, T., "Thermal Conductivity of Two Phase Materials," Industrial and Engineering Chemistry, Vol.53, pp.395-397, 1961.

120. Cheng S., and Vachon, R., "The Prediction of the Thermal Conductivity of Two and Three Phase Solid Heterogeneous Mixtures." International Journal of Heat and Mass Transfer, Vol.12, p249, 1969.

121. Lewis, T., and Nielsen, L., "Dynamic Mechanical Properties of Particulate-Filled Polymers," Journal of Applied Polymer Science, Vol. 14 pp1449-1471, 1970.

122. Progelhof, R., et al. "Methods for Predicting the Thermal Conductivity of Composite Systems: A Review." Polymer Engineering Science, Vol.16, pp.615$625,1976$.

123. Farouki, R., "The Characterization of Parametric Surface Sections." Computer Vision, Graphics and Image Processing, Vol.33, pp 209-236, 1986.

124. Murakami, E., and Okos, M., "Measurement and Prediction of Thermal Properties of Foods." In Food Properties and Computer-Aided Engineering Food Processing Systems, pp3-48, Dordrecht, 1989.

125. Islam, R. and Pramila, A., "Thermal Conductivity of Fiber Reinforced Composites by the FEM," Journal of Composite Materials, Vol.33, pp1699-1715, 1999. 
126. Chawla, k., Composite Materials Science and Engineering, $2^{\text {nd }}$ ed, Springer, New York, 1998.

127. Bejan, A., Convection Heat Transfer, John Wiley \& Sons, Inc., 2004.

128. Agari Y., and Uno, T., "Estimation on Thermal Conductivities of Filled polymers," Journal of Applied Polymer Science, Vol.32, pp.5705-5712, 1986.

129. Rahman, S., Food Properties Handbook, CRC Press, 1995.

130. Jiang, F. and Sousa, A., "Effective Thermal Conductivity of Heterogeneous MultiComponent Materials: an SPH Implementation," Journal of Heat and Mass Transfer, Vol.43, pp479-491, 2007.

131. Madenci, E. and Guven, I., The Finite Element Method and Applications in Engineering Using ANSYS, Springer Science, 2006.

132. Jones, F., and Pascal, F., "Numerical Calculation of the Thermal Conductivities of Composites a 3-D Model." Geophysics, Vol.60, pp. 1038-1050, 1995.

133. Micromechanical Materials Modeling, Delaware Composites Design Encyclopedia, Technomic Publishing Company, Vol.2, 1990.

134. Moaveni, S. Finite Element Analysis: Theory and Applications with ANSYS, $3^{\text {rd }}$ edition, Prentice-Hall, Inc, 2007.

135. Comini, G., Del Giudice, S. and Nonino, C., Finite Element Analysis in Heat Transfer: Basic Formulation and Linear problems, Taylor \& Francis, 1994.

136. Minkowycz, W., and Sparrow, E., Advances in Numerical Heat Transfer, Vol.1, Taylor \& Francis, 1997.

137. Stolarski, T., Nakasone, Y., and Yoshimoto, S., Engineering Analysis with ANSYS Software, Elsevier Butterworth- Heinemann, 2006.

138. Kumlutas, D., and Tavman, I., "A Numerical and Experimental Study on Thermal Conductivity of Particle Filled Polymer Composite," Journal of Thermoplastic Composite Science and Technology, Vol.19, pp.441-455. 2006.

139. Al-sulaiman, F. et al. "Prediction of the Thermal Conductivity of the Constituents of Fiber Reinforced Composites Laminates: Voids Effects," Journal of Composite Materials, Vol.40, pp797-814, 2006.

140. Al-Nassar, Y. "Prediction of Thermal Conductivity of Air Voided- Fiber-Reinforced Composites Laminates part II: 3D Simulation," Journal of Heat and Mass Transfer, Vol.43, pp117-122, 2006 


\section{Appendix: ANSYS Program Code for Thermal Simulation}

! Parametric thermal macro

! Modeling approach: Mesh an entire populated block array,

! Then solve, and then progressively convert core region to air, solve, etc...

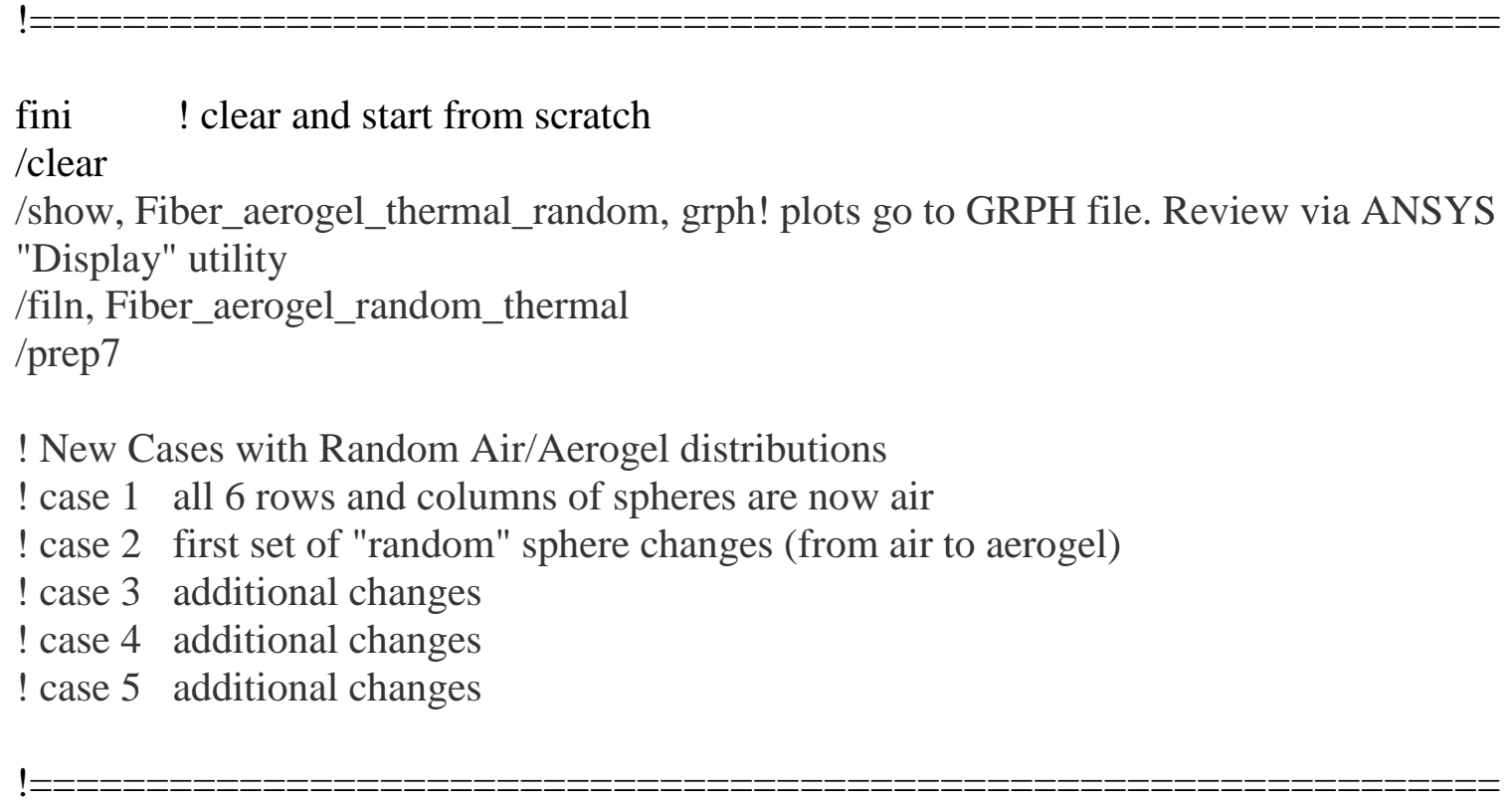

Tlabel='Fiber_Model' ! create an updateable screen annotation

/TSPEC, 15, 1.4, 4, 0, 0

/TLAB, $-0.45,0.82, \%$ Tlabel\%

! Window Settings, semi iso view

/VIEW, 1, $0.19559,0.17157,0.96556$

/ANG, 1, 0.39859 ,ZS

/pnum,mat, 1

/numb, 1

/GRAPHICS,POWER

!----- Thermal Parameters

T_bottom $=200$ ! celsius

T_ambient=25 ! surrounding Air, and initial temperature of array

Hf_top $=100$ ! convective heat trans coef, applied to sides \& top

Hf_sides $=100$

end_time=.2 ! total seconds being simulated, the geom is very small, it doesn't take much time to steadystate. 
step_time=end_time $/ 20$ ! increment in time steps (seconds)

!----- Material Reference Numbers

fiber $=5 \quad$ ! choose the entity number to obtain useful ANSYS colors

aerogel $=3$

Air $=1 \quad$ ! air=1 is good for future Flotran if desired.

!----- Thermal Material Properties, SI units... meters, kg, etc...

mp,kxx,air,.0386

$\mathrm{mp}, \mathrm{kxx}$,fiber, .24

mp,kxx,aerogel,.003

mp,dens,air,.746

mp,dens, fiber, 190

mp,dens, aerogel,.187

mp,c,air, 1.026

mp,c,matrix, 1.2

$\mathrm{mp}, \mathrm{c}$, aerogel,.0703

!----- Geometrical Parameters CONVERT ALL TO METERS FOR CONSISTENCY

meshsize $=1.3 \quad$ ! a meshing parameter to easily coarsen $($ if $>1)$ or refine the mesh $($ if $<1)$. ! If meshsize is greater than 1 then it makes fewer nodes \& elements. saves computer time!

! $\quad$ Set meshsize greater than 1 if your computer is SLOW !

! If meshsize is less than one, then the element edges will be smaller $=>$ makes more elements \& nodes.

! More nodes \& Elements $\Rightarrow$ greater accuracy usually, but takes more time and CPU resources.

! coarse meshes (meshsize > 1) are more likely to fail (or provide unexpected results) due to mesh shape problems

aero_rad $=.35 / 1000 \quad$ ! sphere radius, SI, meters throughout for consistency aero_spc $=2 *$ aero_rad $+2 *(.7 / 1000 / 2)$ ! total size of uniform block surrounding each sphere

NUM_SIDE $=6$ ! number of aerogel parameters in $\mathrm{X}$ direction

nUM_HGT $=6$ ! number of aerogel parameters in Y direction

! Note: alternate parameters may work, but there is no other testing or error checking performed. 


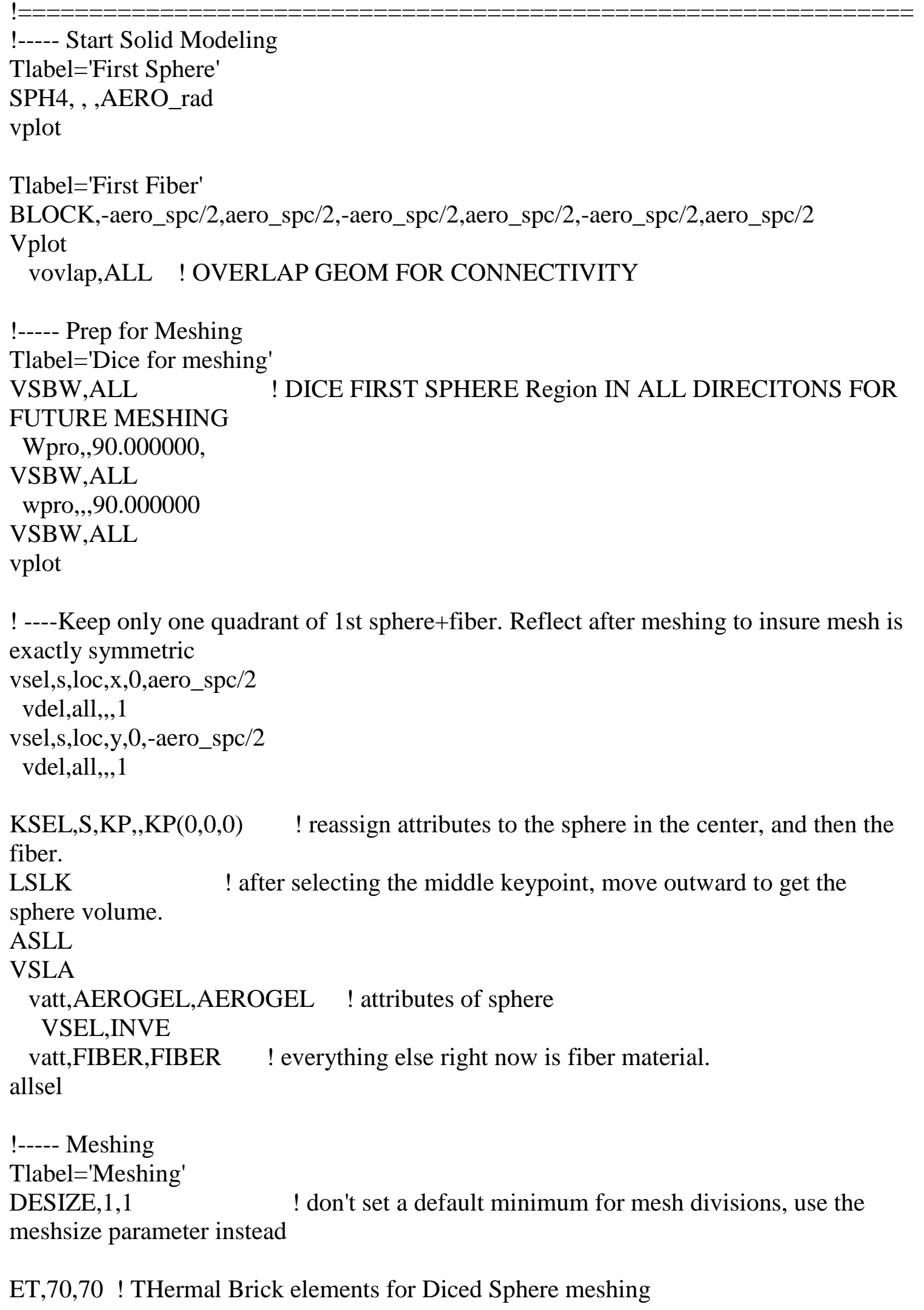


ET,87,87 ! Tets for surrounding irregular Fiber. Brick/Tet, reasonable shape match for thermal simulation.

! For thermal DOF, nodes line up at corners of all elements.

ET,90,90 ! high order thermal solid, for extrusion of outer layer

vsel,s,mat,,aerogel

type, 70

ESIZE,AERO_rad/2*meshsize ! Mesh the Sphere First, default element size for

Sphere

mshape, $0,3 \mathrm{D} \quad$ ! bricks

vmesh,all ! mesh the first sphere with bricks since it is diced \& uniform

vsel,inve ! now, Mesh the surrounding first block of Fiber with tets

! type,87 ! higher order, mid-side nodes

ESIZE,AERO_rad/3*meshsize*1.4 ! default fiber element edge size for matrix

mshape, $1,3 \mathrm{D} \quad$ ! tets

vmesh,all

!----- Reflect the first segment of the Block (with Sphere inside).

! This insures uniform mesh for subsequent copying

allsel

VSYMM,X,all, , , ,0,0 ! horizontal sym reflection of 1 st meshed zone.

VSYMM,Y,all, , , ,0,0 ! Vertical sym reflection of 1st meshed zone.

!----- Copy the first Block (with Sphere inside) around in X \& Y to build the array

Tlabel='Copy Mesh Around'

vgen,NUM_SIDE,all,,,aero_spc

VGEN,NUM_HGT,ALL, , , ,-AERO_SPC

nummrg,kp,aero_rad/100,aero_rad/100 ! merge coincident geom for connectivity

nummrg,node,aero_rad/100

nummrg,kp,aero_rad/100,aero_rad/100 ! merge coincident geom for connectivity

nummrg,node,aero_rad/100

EsEL,S,MAT,,FIBER

/TRLCY,ELEM,0.8, all ! make the Fiber look a little transparent

ALLSEL

eplot

! Extrude out every Fiber side additional amount to match specified .

! ----- DETERMINE OVERALL MODEL SIZE IN ACTIVE CSYS

*get,kpmnx,kp,,mnloc, $\mathrm{x}$ ! get max and min $\mathrm{x}$ kp locations in this csys 


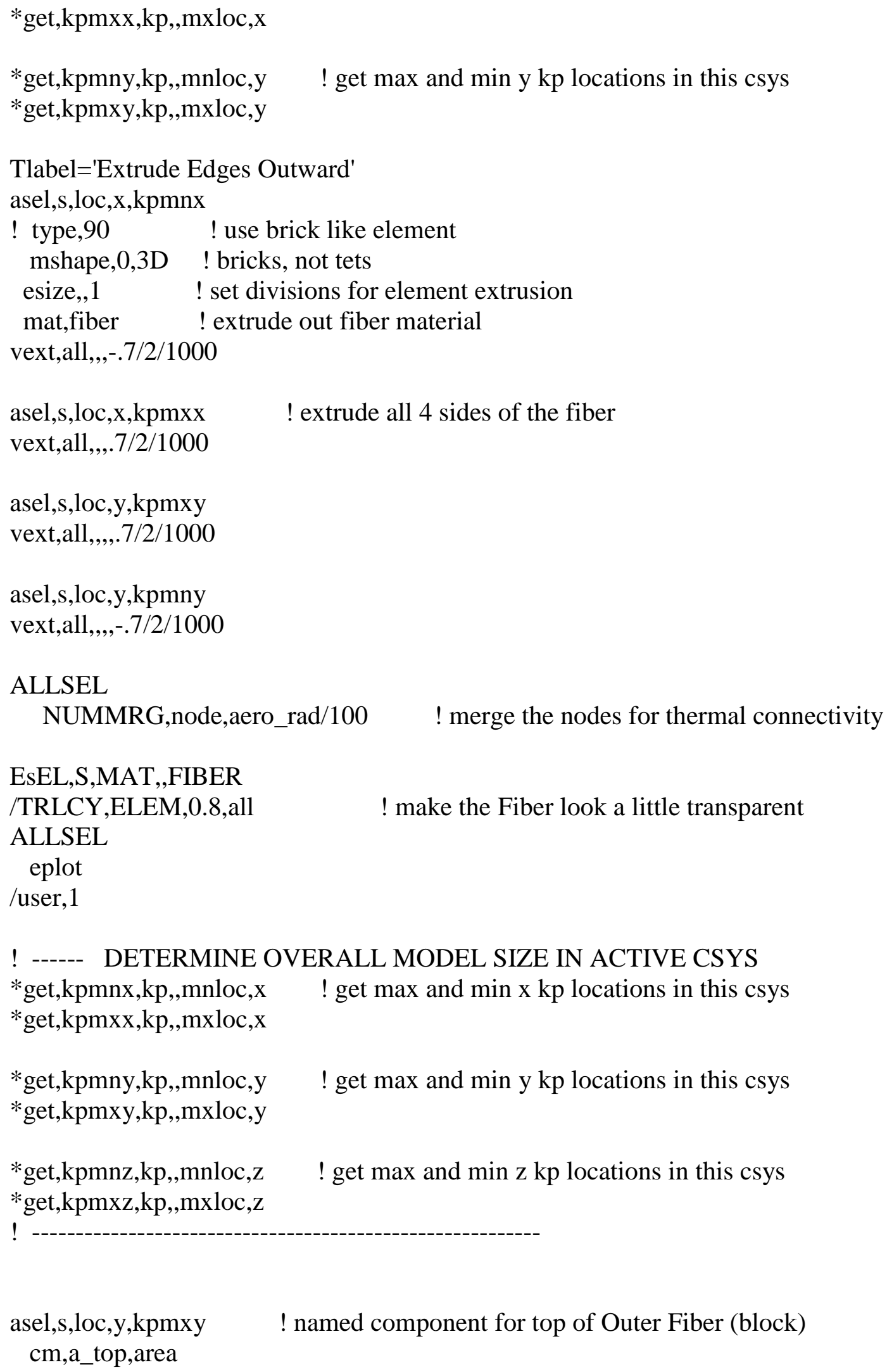




\begin{tabular}{|c|c|}
\hline $\begin{array}{c}\text { asel,s,loc,y,kpmny } \\
\text { cm,a_bot,area }\end{array}$ & ! named component for bottom of Outer Fib \\
\hline $\begin{array}{c}\text { asel,s,loc,x,kpmxx } \\
\text { asel,a,loc,x,kpmnx } \\
\text { cm,a_sides,area }\end{array}$ & ! sides of the outer Fiber \\
\hline $\begin{array}{c}\text { asel,s,loc,z,kpmxz } \\
\text { asel,a,loc,z,kpmnz } \\
\text { cm,a_ends,area }\end{array}$ & ! named component for ends of fiber block \\
\hline
\end{tabular}

! put a coord system at the bottom of the lowest spheres vsel,s,mat,,aerogel

allsel,below,volu

*get,kpmnyb,kp,,mnloc,y ! get max and min y kp locations in this csys

WPCSYS, -1,0

wpave,kpmnx,kpmnyb,kpmnz,kpmxx,kpmnyb,kpmxz cswp, 100,0

fini

/fdele,full,dele ! instruct ANSYS to delete some of the larger files when done /fdele,rdb,dele

/fdele,esav,dele

/soln allsel

antype,trans ! transient analysis, includes thermal inertia based on Density and "C" Heat Capacity acel,,9.81, ! gravity, in case it becomes a consideration

Tlabel='ambient convection' sfa,a_top,,conv,hf_top,T_ambient !----- Apply Convection Boundaries on top and sides sfa,a_sides,,conv,hf_sides,T_ambient

cmsel,s,a_top ! select those surfaces in order to show the convection surfaces cmsel,a,a_sides 
/PSF,CONV,HCOE,3,0,1

aplot

! Plot the convection surfaces

/PSF,DEFA, ,3,0,1
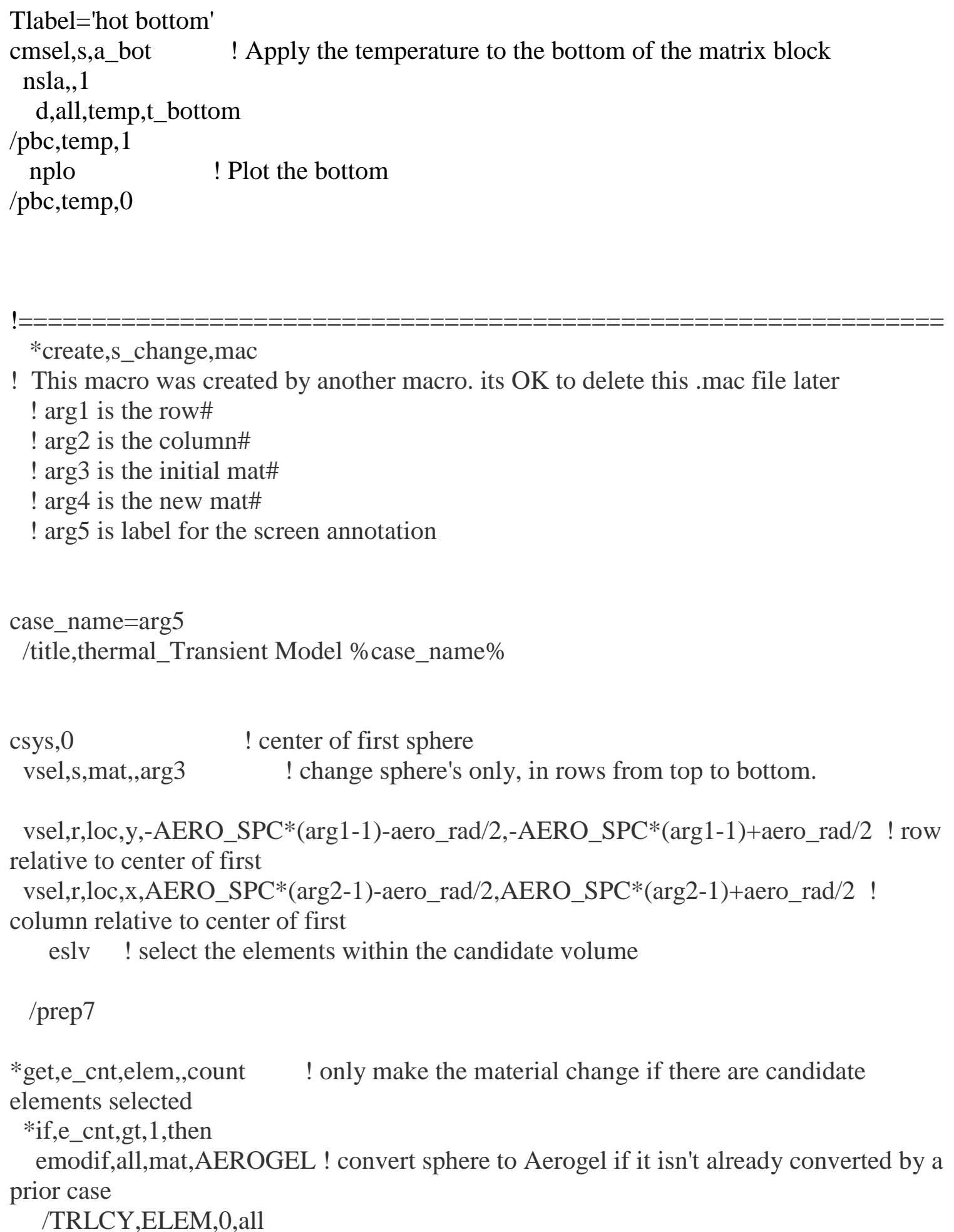
*endif

Tlabel=arg5 $\quad$ ! updated the screen annotation for this Case

fini

/title,thermal_Transient \%arg5\%

allsel

*end ! ends s_change macro

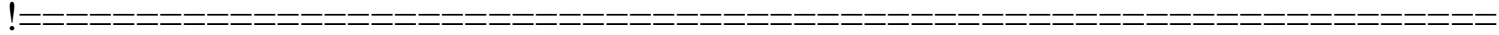

*create,do_solve,mac

! This macro was created by another macro. It is OK to delete this .mac file later

! $\arg 1$ is case name

case_name $=\arg 1$

/title,thermal_Transient Model \%case_name\%

allsel

eplot

Tlabel='this case: spheres'

vsel,s,mat,,air

eslv

nsle

eplot

Tlabel='this case: aerogel' ! Plot the various material groups this Case esel,s,mat,,aerogel

*get,e_cnt,elem,,count

*if,e_cnt,gt, 1 , then

eplot ! plot aerogel's if there are any

*endif

Tlabel='this case: air'

esel,s,mat, air

eplot

Tlabel='this case: fiber'

esel,s,mat,,fiber

eplot

/title,thermal_Transient Results \%case_name\%

/soln 


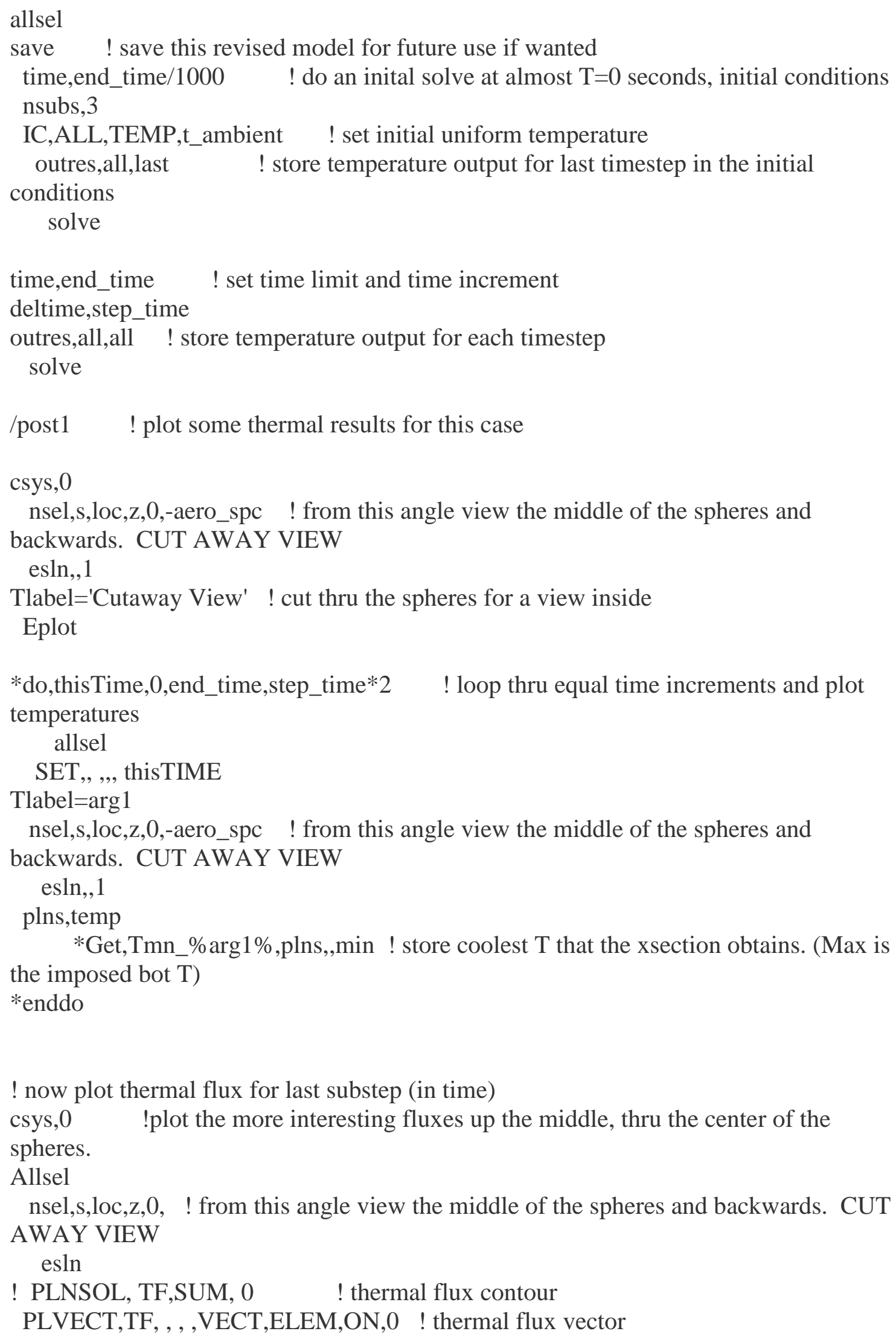


*end ! ends creation of do_solve run macro

! -----------------------------------------------------------

! case 1 all 6 layers of spheres are now air

! cASE 2 A Random group becomes Aerogel

! case 3 Additional Random group becomes Aerogel

! case 4 Additional Random group becomes Aerogel

! case 5 Additional Random group becomes Aerogel

!----- Loop thru the cases. Due to the mesh mods being made, we solve the cases backwards from 5 to 1 .

! it's OK to repeat row,column designation. Nothing new will happen if the sphere is already converted.

! S_change format is row,column,starting material, ending material,lable

! do_solve format is s_change,label

! Random sphere CASE

!Case 1 all spheres are AIR

do_solve,'Case1' ! all spheres AIR

! cASE 2 Random group becomes Aerogel

!Case 2 convert row,column Air sphere to aerogel

s_change, 1,3,AIR,AEROGEL,'CASE2'

s_change, 1,4,AIR,AEROGEL,'CASE2'

s_change, 2,3,AIR,AEROGEL,'CASE2'

s_change, 3,5,AIR,AEROGEL,'CASE2'

do_solve,'Case2'

! case 3 Additional Random group becomes Aerogel

!Case 3 convert row,column Air sphere to aerogel

s_change, 1,3,AIR,AEROGEL,'CASE3'

s_change, 1,4,AIR,AEROGEL,'CASE3'

s_change, 2,3,AIR,AEROGEL,'CASE3'

s_change, 3,5,AIR,AEROGEL,'CASE3'

s_change,1,2,AIR,AEROGEL,'CASE3'

s_change, 2,4,AIR,AEROGEL,'CASE3'

s_change, 2,6,AIR,AEROGEL,'CASE3'

s_change,3,4,AIR,AEROGEL,'CASE3' 
s_change,4,6,AIR,AEROGEL,'CASE3'

s_change,5,1,AIR,AEROGEL,'CASE3'

s_change, 6,5,AIR,AEROGEL,'CASE3'

do_solve,'Case3'

!

! case 4 Additional Random group becomes Aerogel

!Case 4 convert row,column Air sphere to aerogel

s_change, 1,3,AIR,AEROGEL,'CASE4'

s_change,1,4,AIR,AEROGEL,'CASE4'

s_change, 2,3,AIR,AEROGEL,'CASE4'

s_change,3,5,AIR,AEROGEL,'CASE4'

s_change,1,1,AIR,AEROGEL,'CASE4'

s_change,1,2,AIR,AEROGEL,'CASE4'

s_change,1,5,AIR,AEROGEL,'CASE4'

s_change, 2,2,AIR,AEROGEL,'CASE4'

s_change, 2,4,AIR,AEROGEL,'CASE4'

s_change, 2,6,AIR,AEROGEL,'CASE4'

s_change,3,2,AIR,AEROGEL,'CASE4'

s_change,3,4,AIR,AEROGEL,'CASE4'

s_change,4,1,AIR,AEROGEL,'CASE4'

s_change,4,3,AIR,AEROGEL,'CASE4'

s_change, 4,6,AIR,AEROGEL,'CASE4'

s_change,5,1,AIR,AEROGEL,'CASE4'

s_change,5,6,AIR,AEROGEL,'CASE4'

s_change, 6,5,AIR,AEROGEL,'CASE4'

do_solve,'Case4'

! case 5 Additional Random group becomes Aerogel

!Case 5 convert row, column Air sphere to aerogel

s_change,1,3,AIR,AEROGEL,'CASE5'

s_change,1,4,AIR,AEROGEL,'CASE5'

s_change, 2,3,AIR,AEROGEL,'CASE5'

s_change,3,5,AIR,AEROGEL,'CASE5'

s_change,1,1,AIR,AEROGEL,'CASE5'

s_change,1,2,AIR,AEROGEL,'CASE5'

s_change, 1,5,AIR,AEROGEL,'CASE5'

s_change, 1,6,AIR,AEROGEL,'CASE5'

s_change, 2,2,AIR,AEROGEL,'CASE5'

s_change, 2,4,AIR,AEROGEL,'CASE5'

s_change, 2,5,AIR,AEROGEL,'CASE5'

s_change, 2,6,AIR,AEROGEL,'CASE5'

s_change,3,1,AIR,AEROGEL,'CASE5' 
s_change, 3,2,AIR,AEROGEL,'CASE5'

s_change, 3,3,AIR,AEROGEL,'CASE5'

s_change, 3,4,AIR,AEROGEL,'CASE5'

s_change, 4,1,AIR,AEROGEL, 'CASE5'

s_change, 4,3,AIR,AEROGEL,'CASE5'

s_change,4,4,AIR,AEROGEL,'CASE5'

s_change,4,5,AIR,AEROGEL,'CASE5'

s_change, 4,6,AIR,AEROGEL,'CASE5'

s_change, 5,1,AIR,AEROGEL,'CASE5'

s_change,5,2,AIR,AEROGEL,'CASE5'

s_change,5,4,AIR,AEROGEL,'CASE5'

s_change,5,6,AIR,AEROGEL,'CASE5'

s_change, 6,2,AIR,AEROGEL,'CASE5'

s_change, 6,3,AIR,AEROGEL,'CASE5'

s_change, 6,4,AIR,AEROGEL,'CASE5'

s_change,6,5,AIR,AEROGEL,'CASE5'

do_solve,'Case5'

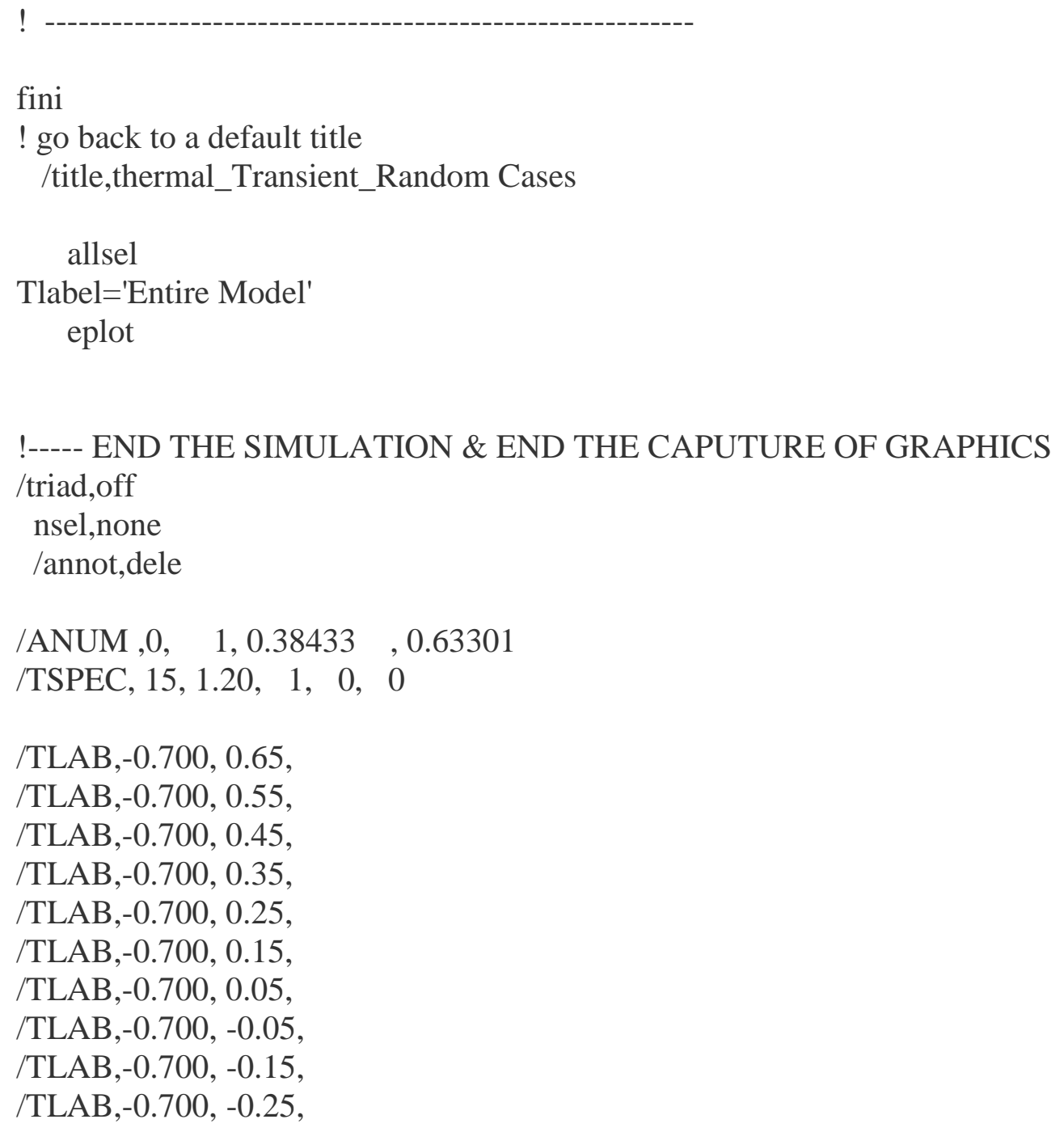




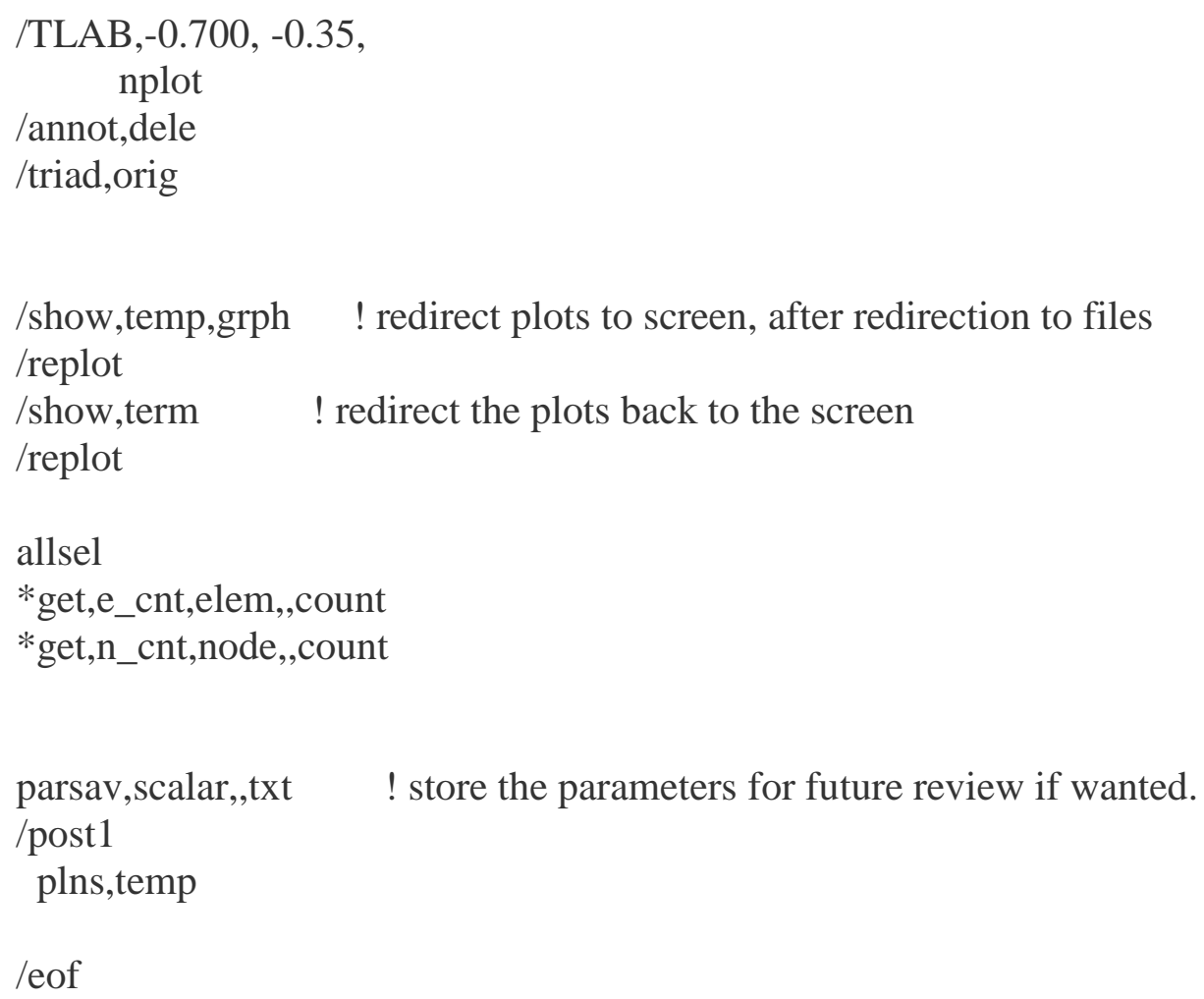

